

\section{Growth and thermal oxidation of Ru and $\mathrm{ZrO}_{2}$ thin films as oxidation protective layers}

Roger Coloma Ribera 


\section{Graduation committee:}

\section{Chairman:}

Prof. dr. ir. J. W. M. Hilgenkamp

University of Twente, TNW

\section{Secretary:}

Prof. dr. ir. J. W. M. Hilgenkamp

University of Twente, TNW

\section{Promotor:}

Prof. dr. F. Bijkerk

University of Twente, TNW

\section{Co-promotor:}

Dr. ir. R. W. E. van de Kruijs

University of Twente, TNW

\section{Members:}

Prof. dr. D. Depla

Ghent University
Eindhoven University of Technology
University of Twente, EWI
University of Twente, TNW
University of Twente, TNW

Prof. dr. H. H. Brongersma

Prof. dr. J. Schmitz

University of Twente, TNW

Cover: The image in the front cover shows the Atomic Growth and Analysis (AGA) cluster that was used for most of the experiments described in this thesis. The small images on the back side show, from top to bottom: magnetron sputtering deposition, LEIS analysis, XPS analysis, and grazing-incidence XRR analysis. Photographs by Gijs van Ouwerkerk (AGA, XPS), Koen Weghorst (deposition), Marko Sturm (LEIS), and Ani Chandrasekaran (XRR).

Growth and thermal oxidation of $\mathrm{Ru}$ and $\mathrm{ZrO}_{2}$ thin films as oxidation protective layers Roger Coloma Ribera

$\mathrm{PhD}$ thesis, University of Twente, Enschede, The Netherlands

With references - With summary in English, Dutch and Spanish.

ISBN: 978-90-365-4310-1

DOI: $10.3990 / 1.9789036543101$

Published by the Industrial Focus Group XUV Optics, University of Twente, Enschede, The Netherlands

Printed by Ipskamp Printing, Enschede, The Netherlands

(C) Roger Coloma Ribera (2017)

All rights reserved. 


\title{
GROWTH AND THERMAL OXIDATION OF RU AND ZRO2 THIN FILMS AS OXIDATION PROTECTIVE LAYERS
}

\author{
DISSERTATION
}

\author{
to obtain \\ the degree of doctor at the University of Twente, \\ on the authority of the rector magnificus \\ Prof. dr. T. T. M. Palstra \\ on account of the decision of the graduation committee, \\ to be publicly defended \\ on Wednesday $1^{\text {st }}$ March, 2017 at 16:45 \\ by \\ Roger Coloma Ribera
}

born on $31^{\text {st }}$ October 1986

in Sant Joan Despí, Barcelona, Spain 
This dissertation has been approved by the promotor:

Prof. dr. F. Bijkerk

and the co-promotor:

Dr. ir. R. W. E. van de Kruijs

ISBN: 978-90-365-4310-1

(C) Roger Coloma Ribera (2017) 
The work presented in this thesis is based on the following publications:

\section{Chapter 3:}

R. Coloma Ribera, R. W. E. van de Kruijs, S. Kokke, E. Zoethout, A. E. Yakshin, and F. Bijkerk, "Surface and sub-surface thermal oxidation of thin ruthenium films," Applied Physics Letters 105 (13), 131601 (2014).

\section{Chapter 4:}

R. Coloma Ribera, R. W. E. van de Kruijs, A. E. Yakshin, and F. Bijkerk, "Determination of oxygen diffusion kinetics during thin film ruthenium oxidation," Journal of Applied Physics 118 (5), 055303 (2015).

\section{Chapter 5:}

R. Coloma Ribera, R. W. E. van de Kruijs, J. M. Sturm, A. E. Yakshin, and F. Bijkerk, "In vacuo growth studies of $\mathrm{Ru}$ thin films on $\mathrm{Si}, \mathrm{SiN}$, and $\mathrm{SiO}_{2}$ by high-sensitivity low energy ion scattering," Journal of Applied Physics 120 (6), 065303 (2016).

\section{Chapter 6:}

R. Coloma Ribera, R. W. E. van de Kruijs, J. M. Sturm, A. E. Yakshin, and F. Bijkerk, "Intermixing and thermal oxidation of $\mathrm{ZrO}_{2}$ thin films grown on a-Si, $\mathrm{SiN}$ and $\mathrm{SiO}_{2}$ by metallic and oxidic mode magnetron sputtering," submitted to Journal of Applied Physics.

\section{CP3嗐

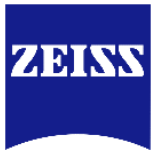 \\ ASML 言言部言 MESA+ UNIVESSTY OF TWENTE.

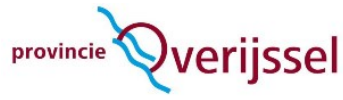

This work is part of the research programme 'Controlling photon and plasma induced processes at EUV optical surfaces (CP3E)' of the 'Stichting voor Fundamenteel Onderzoek der Materie (FOM)', which is part of and financially supported by the 'Nederlandse Organisatie voor Wetenschappelijk Onderzoek (NWO)'. The CP3E programme is also co-financed by Carl Zeiss SMT and ASML, and carried out in the Industrial Focus Group XUV Optics, which belongs to the MESA+ Institute for Nanotechnology at the University of Twente. The infrastructure for this programme is also financially supported by the Province of Overijssel. 



\section{Table of Contents}

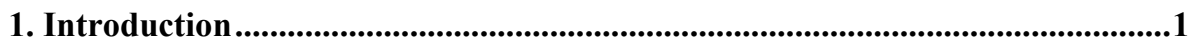

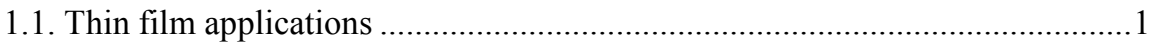

1.1.1. Metal thin films ..............................................................................

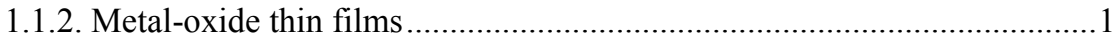

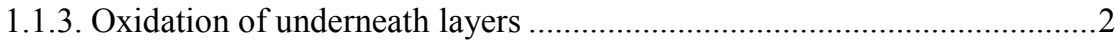

1.2. Oxidation resistant protective layers .............................................................

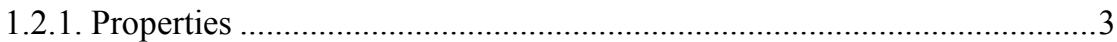

1.2.2. Material selection criteria based on oxidation properties ...........................

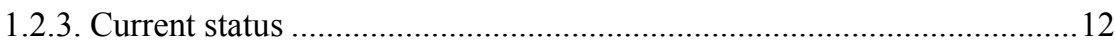

1.2.3.1. Microelectronic (DRAM and CMOS) devices ..................................12

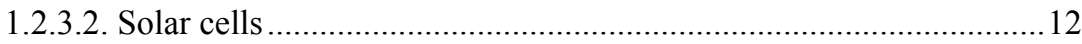

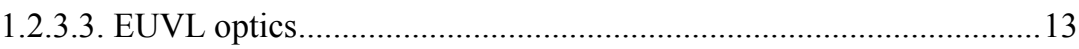

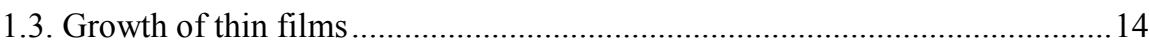

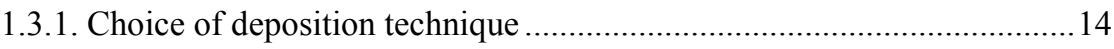

1.3.2. Room-temperature magnetron sputtering ................................................ 16

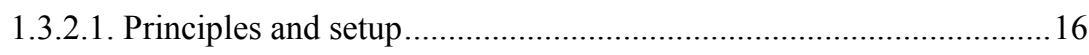

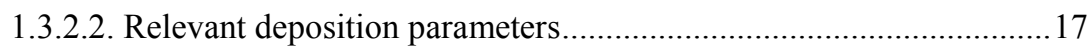

1.3.2.3. Relevant processes occurring during deposition ...............................19

1.3.3. Current status of growth of metal and metal-oxide thin films..................21

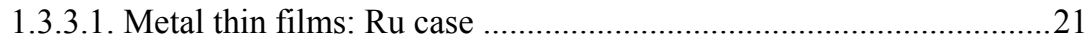

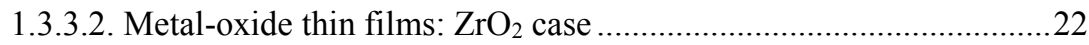

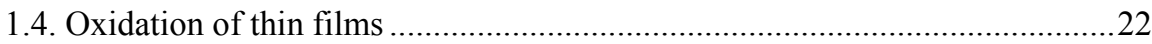

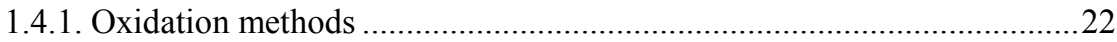

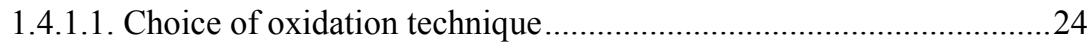

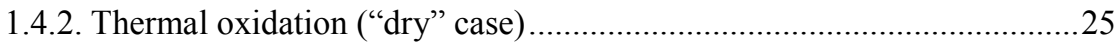

1.4.2.1. Main processes occurring during oxidation $\left(\mathrm{O}_{2}\right)$ of metals ..............25

1.4.3. Diffusion-limited growth of oxide thin films ............................................ 31

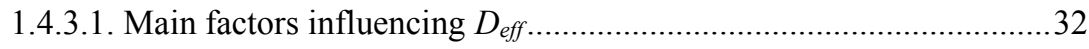

1.4.3.2. Current status of Ru thin films oxidation: diffusion-limited growth

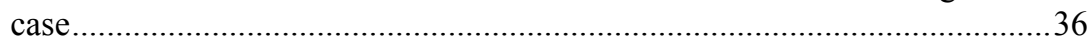

1.4.3.3. Current status of oxygen diffusion through $\mathrm{RuO}_{2}$ and $\mathrm{ZrO}_{2}$ films ...37

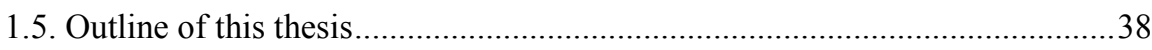


2. Experimental …..................................................................................................41

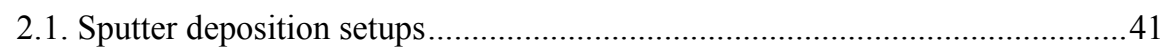

2.1.1. Advanced Development Coater (ADC) …………………………..........41

2.1.2. Atomic Growth and Analysis (AGA) .....................................................4

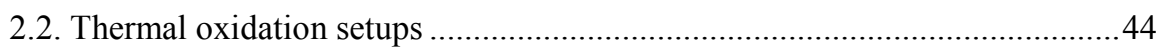

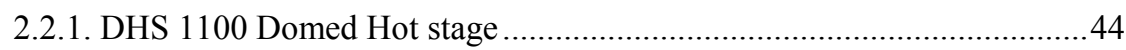

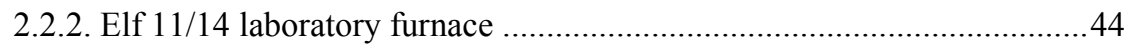

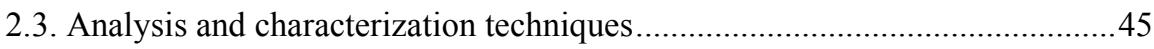

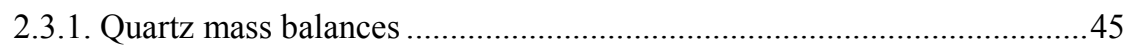

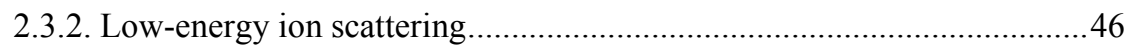

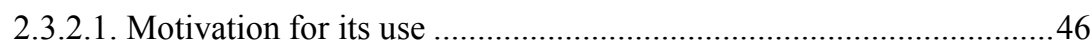

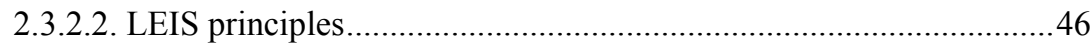

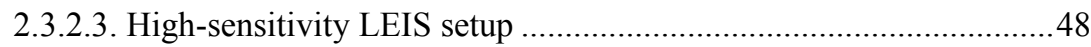

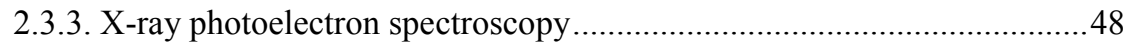

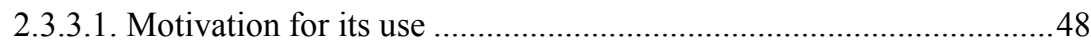

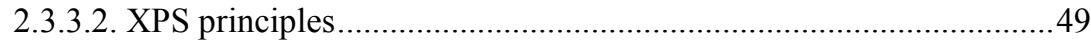

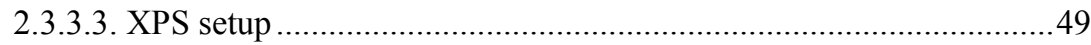

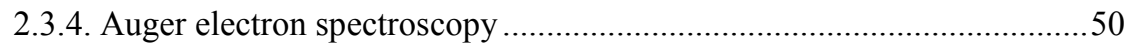

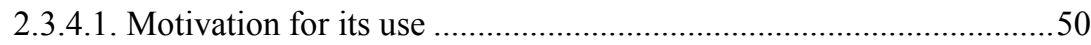

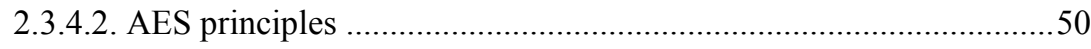

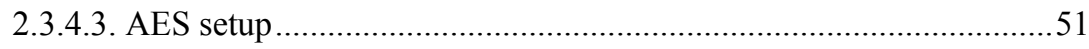

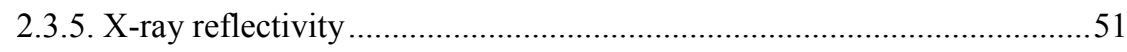

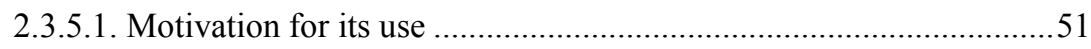

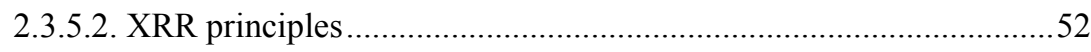

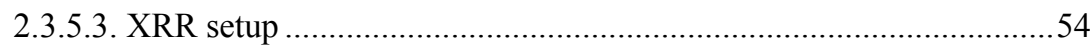

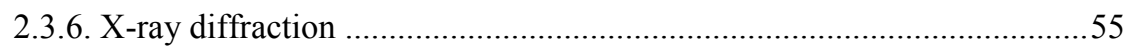

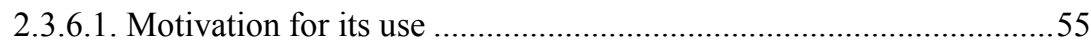

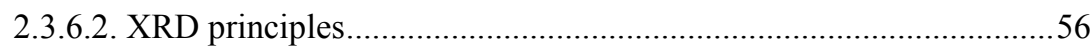

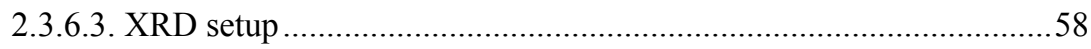

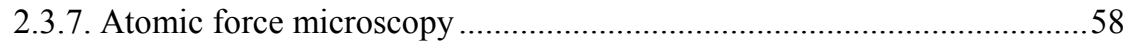

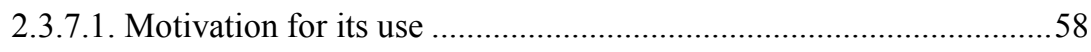

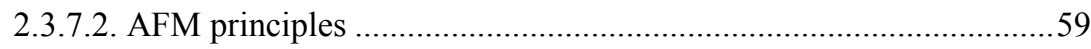

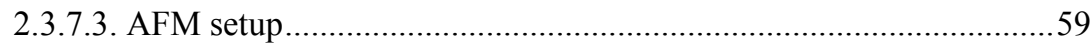




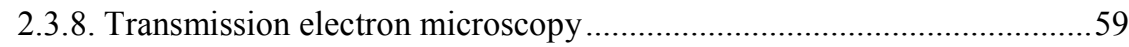

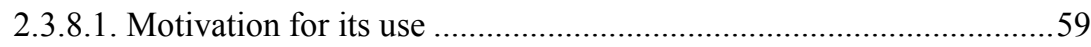

2.3.8.2. TEM principles.......................................................................... 60

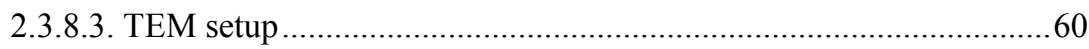

3. Surface and sub-surface thermal oxidation of thin ruthenium films...............61

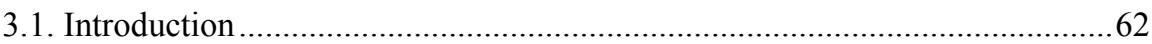

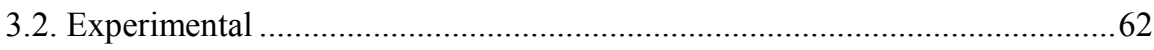

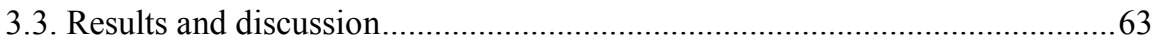

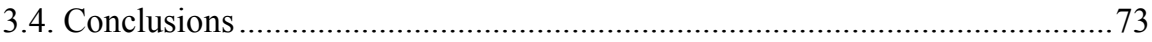

4. Determination of oxygen diffusion kinetics during thin film ruthenium

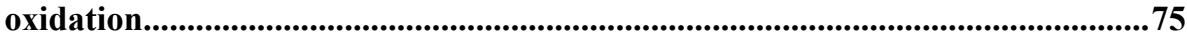

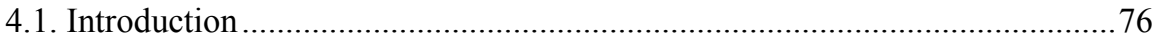

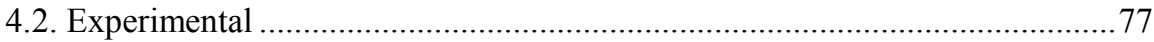

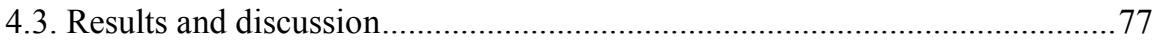

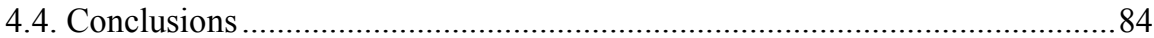

Appendix A. Determination of the uncertainties of the activation energies and pre-exponential factors for oxygen diffusion in $\mathrm{RuO}_{2}$ thin films ..........................85

5. In vacuo growth studies of $\mathrm{Ru}$ thin films on $\mathrm{Si}, \mathrm{SiN}$ and $\mathrm{SiO}_{2}$ by highsensitivity low energy ion scattering .............................................................8.

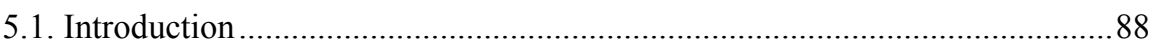

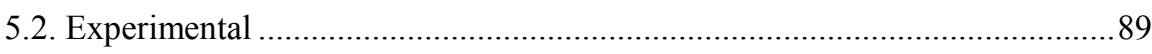

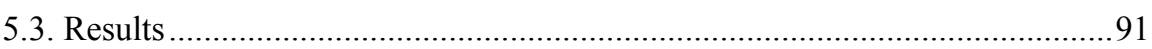

5.3.1. Surface coverages and closed layer determination for $\mathrm{Ru}$ on $\mathrm{Si}, \mathrm{SiN}$

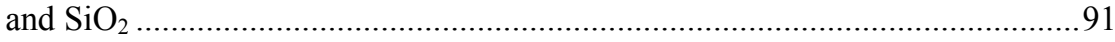

5.3.2. In-depth Ru profiles from Ru surface coverages ............................... 100

5.3.3. Surface coverages, closed layer determination and in-depth Si profile

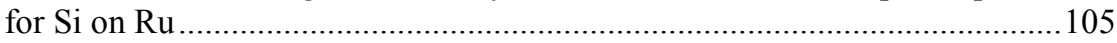

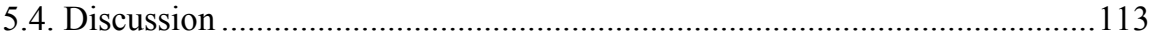

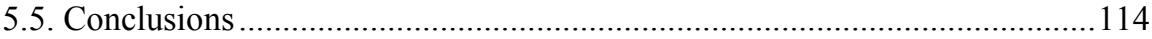

6. Intermixing and thermal oxidation of $\mathrm{ZrO}_{2}$ thin films grown on a-Si, $\mathrm{SiN}$ and $\mathrm{SiO}_{2}$ by metallic and oxidic mode magnetron sputtering ...........................115

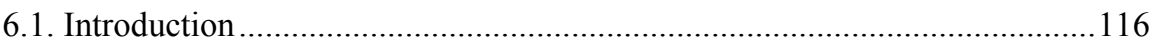

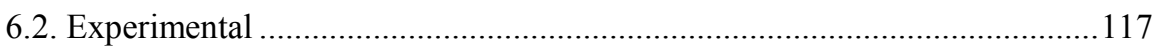

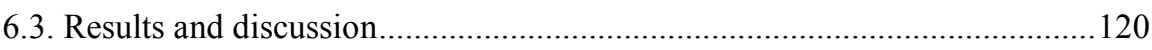

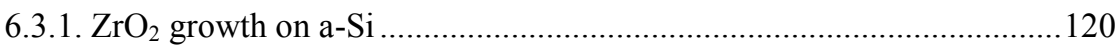

6.3.2. Effect of Si passivation on $\mathrm{ZrO}_{2}$ growth ...........................................130 
6.3.3. Oxygen diffusion response upon thermal oxidation of the $\mathrm{ZrO}_{2} / \mathrm{Si}$

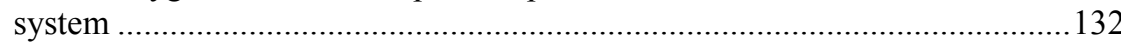

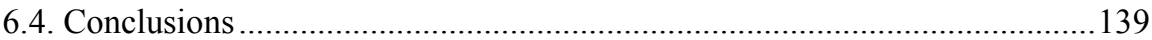

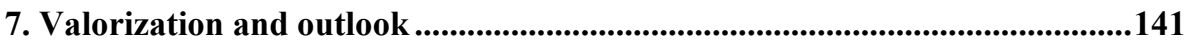

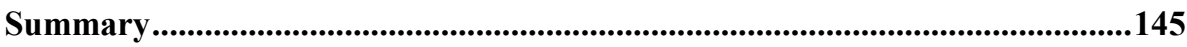

Samenvatting .......................................................................................................149

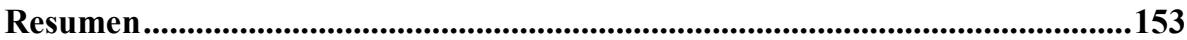

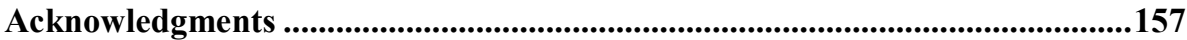

Curriculum Vitae .............................................................................................................159

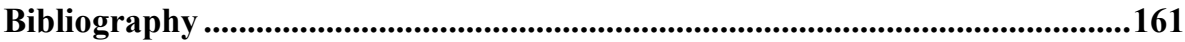




\section{Introduction}

\subsection{Thin film applications}

In the last decades, nanometer thin films have been used in numerous applications due to their interesting scale-related properties, mostly different from typical bulk properties, and highly dependent on the material type (i.e., metal, oxide, nitride, etc.). The main applications for the first two material type thin films are described in the next sections (Secs. 1.1.1 and 1.1.2).

\subsubsection{Metal thin films}

Metal (e.g., Pd, Ru, etc.) thin films have been applied in several fields (i.e., catalysis, electronics and optics) due to their relevant associated properties. For instance, $\mathrm{Ru}$ has turned out to be one of the most active catalysts in ammonia synthesis. ${ }^{1,2}$ Due to its low resistivity and low solubility with $\mathrm{Cu},{ }^{3}$ it has also been used as $\mathrm{Cu}$ diffusion barrier and/or $\mathrm{Cu}$ seed layer in integrated circuits with copper interconnect technology. ${ }^{4}$ Other applications for $\mathrm{Ru}$ are as bottom electrode in capacitors based on high dielectric materials, ${ }^{5,6}$ or as capping layer for optics designed for extreme ultraviolet lithography (EUVL) $^{7,8,9,10}$, due to its low-oxidation properties. ${ }^{11}$

\subsubsection{Metal-oxide thin films}

Metal-oxide (e.g., $\mathrm{RuO}_{2}, \mathrm{ZrO}_{2}$, etc.) thin films not only have been applied in similar fields as the ones mentioned in Sec. 1.1.1 for metallic films, but also in photovoltaics or other applications, due to their characteristic oxide properties. For instance, $\mathrm{RuO}_{2}$, due to its excellent catalytic performance, ${ }^{12,13}$ can be used as oxidation catalyst in heterogeneous catalysis and electrocatalysis. ${ }^{14}$ Due to its low resistivity, ${ }^{15}$ high thermal stability, ${ }^{16}$ good diffusion barrier capability, ${ }^{17}$ and easy patterning, ${ }^{18} \mathrm{RuO}_{2}$ has also been applied as bottom gate electrode of oxide dielectric capacitors in dynamic random access memories (DRAMs), ${ }^{6}$ or as protective capping layer in EUV lithography. ${ }^{7,9,10}$ As another example, $\mathrm{ZrO}_{2}$ thin films present several attractive properties including: high thermal and chemical stability, ${ }^{19,20,21}$ high dielectric constant, ${ }^{22,23}$ large band gap, ${ }^{24}$ high hardness, ${ }^{25}$ and good optical performance in the near UV-Vis region, ${ }^{26,27,28}$ which make 
them suitable for many applications. For instance, $\mathrm{ZrO}_{2}$ can be used in optical devices such as UV filters and laser mirrors. ${ }^{29,30,31}$ Apart from other relevant properties, its low reactivity with $\mathrm{Si}^{32}$ has made $\mathrm{ZrO}_{2}$ an alternative high-K material to $\mathrm{SiO}_{2}$ used as gate dielectric for complementary metal-oxide-semiconductor (CMOS) devices, ${ }^{22,33}$ and as dielectric layer for volatile DRAMs. ${ }^{34,35}$ This low reactivity of $\mathrm{ZrO}_{2}$ with $\mathrm{Si}$, together with the fact that $\mathrm{Zr}$ forms a very stable and inert oxide to possible external contaminants, ${ }^{36,37,38}$ have made $\mathrm{ZrO}_{2}$ also a promising candidate as contact and passivation layer for solar cells, ${ }^{39}$ or as capping layer for optics designed for EUV lithography. ${ }^{40,41,42}$

\subsubsection{Oxidation of underneath layers}

In several of the applications described in Secs. 1.1.1 and 1.1.2, such as DRAMs, CMOS devices, solar cells, or optics designed for EUV lithography, oxygen diffusion towards deeper layers is one of the main threats for their performance.

Both memory and CMOS devices are usually fabricated at high temperatures under a strong oxidative environment. In such extreme conditions, the poly-Si plug attached to the high-K dielectric layer is oxidized resulting in a decrease of the effective dielectric constant. ${ }^{22,43}$ However, not only extreme oxidative conditions might compromise the functionality of these devices, but also any annealing treatments which have an excess of oxygen present (either from the ambient or the sidewall oxide, for instance), will lead to a quick oxygen diffusion through the top dielectric layer towards the underneath $\mathrm{Si}$, resulting in the formation of $\mathrm{SiO}_{2}$ or $\mathrm{SiO}_{2}$-containing interfacial layers. The uncontrolled formation of $\mathrm{SiO}_{2}$ at the interface will dramatically compromise the capacitance gain from any high-K dielectric layer in the gate stack, and hence, affect the final response of the electronic device. ${ }^{22}$

Photovoltaics are continuously exposed to external atmospheric contaminants such as liquid and vapor water, or even oxygen gas. This, together with the regular incidence of UV photons from the sun during day time, provokes degradation of the solar cells, mainly due to atmospheric corrosion (or wet oxidation) by uncontrolled defective $\mathrm{SiO}_{2}$ formation, ${ }^{44}$ which is normally enhanced by the cyclical thermal stress present. ${ }^{45}$ This uncontrolled phenomenon causes significant recombination losses at the surface of the solar cells, ${ }^{46}$ leading to a possible failure of the photovoltaic device. ${ }^{47}$ Relatively high temperatures (above $1000^{\circ} \mathrm{C}$ ) are usually required for the formation of an on purpose generated high-quality $\mathrm{SiO}_{2}$ passivation layer, which might result into the degradation of the bulk life time of the cell and the stability of this passivation layer. ${ }^{48}$ Moreover, this method of passivation is commercially unviable due to the enhanced heating costs associated. Besides, a $\mathrm{SiO}_{2}$ passivation layer has very low refractive index and is not very conductive, which makes it unsuitable for a good antireflection performance (for 
the front interface) and a good carrier collection efficiency (mostly related to the back interface). These two properties are needed for the application, and cannot be fulfilled using a $\mathrm{SiO}_{2}$ passivation layer. ${ }^{47}$

Similarly, during the assembly of EUV lithographic systems, the outer surface of the Si/Mo multilayer mirrors (MLMs) used in this application is usually exposed to air, causing oxidation of the last layer of the mirror ( $\mathrm{Si}$ or Mo), and a subsequent reflectance loss. Si forms a thin ( 1-2 nm) self-terminated native, stable oxide which does not evolve at atmospheric conditions. ${ }^{49,50}$ However, Mo forms a thicker (several nanometers) oxide that continues oxidizing upon air exposure. ${ }^{8,51}$ Thus, up to date, multilayer mirrors are normally terminated by $\mathrm{Si}^{52}$ Since EUV radiation is highly absorbed by almost all materials, including air, EUV mirrors and the entire EUVL scanners are kept under ultrahigh vacuum (UHV) conditions (e.g., $<10^{-7}$ mbar). Still, residual background gases are present in the UHV system such as water vapor (e.g., $<10^{-8}$ mbar). ${ }^{36}$ The use of EUV light may induce the cracking of water vapor, generating highly reactive oxygen species. These species may lead to optics contamination by oxidation. ${ }^{41,53}$ As a result, the reflectance of these mirrors may drop, shortening their lifetime. ${ }^{54}$

In all four mentioned applications: DRAMs, CMOS devices, photovoltaics, and EUVL optics, the layer underneath (usually $\mathrm{Si}$ ) should not be directly exposed to an oxidative environment. Thus, there is a strong necessity of protecting this underlying layer by thin (few nanometers) oxidation resistant protective layers, which can prevent in-depth oxidation from occurring. In this thesis, relevant oxidation resistant protective layers are studied for protecting electronics, photovoltaics and EUVL optics against oxidation and/or oxygen diffusion. In the next section (Sec. 1.2), properties, material selection based on oxidation properties, and current status of these protective layers will be discussed in more detail.

\subsection{Oxidation resistant protective layers}

\subsubsection{Properties}

Oxidation resistant protective layers for applications such as electronics (DRAMs, CMOS devices), photovoltaics and EUVL optics, must possess a large amount of properties. Most of these properties make them also suitable for other applications, as already mentioned in Sec. 1.1. Focusing on these four applications, the most relevant properties that a protective layer must possess are presented as follows:

1. Closed layer. This property is associated to the continuity of the protective film. ${ }^{54}$ It should have at least sufficient thickness (normally few nanometers) such that the film is continuous (closed layer thickness) and does not allow the layer underneath (for instance, $\mathrm{Si}$ ) to be in contact with the environment. 
2. Chemically inert. A larger chemical interaction with the underlying layer (typically $\mathrm{Si}$ ) would require an increased thickness of the protective material to close the layer, which would compromise the performance of the device. In electronics, this thickness increase will usually result in a drop of capacitance. ${ }^{22}$ In solar cells, this will lead to a common reduction of the UV transmission, namely through reflection and parasitic absorption, and a loss of the photo-generated power via recombination at trap states and electrical resistance losses. ${ }^{45}$ And, in EUVL optics, this will cause a higher reflectance loss due to EUV absorption. ${ }^{54}$ Thus, formation of interlayer compounds such as silicides or silicates will call for Si-terminated electronic, photovoltaic and optical devices. In addition, surface segregation effects might also contribute to the intermixing for such devices. Sometimes, an extra diffusion barrier between the protective material and the underlying layer is required. Usually this is a better option for the capacitance, the transmission, the photo-generated power, or the reflectance, than allowing a huge interface formation due to intermixing. For instance, TaN barriers are sandwiched between the $\mathrm{Ru}$ protective layer and the Si substrate in integrated circuits with copper interconnect technology. ${ }^{55}$ Another example is the use of $\mathrm{B}_{4} \mathrm{C}$ barriers when $\mathrm{Ru}$ is applied as protective layer on Si-terminated MLMs. ${ }^{49}$

3. Low surface roughness. The root mean square roughness (RMS) of the protective layer should be close to the underneath layered stack and the substrate $(\sim 0.2$ $0.3 \mathrm{~nm}$ ), not to add extra roughness, compromising the performance of the overall electronic, ${ }^{56,57}$ photovoltaic, ${ }^{45}$ or optical system. ${ }^{54}$ In addition, a high roughness suggests "low quality" protective layer growth, often connected to a porous structure and enhanced oxygen diffusion towards the underlying layers.

4. Low temperature deposition. This protective material must be able to be deposited at relatively low growth temperatures to avoid higher intermixing with the layer underneath and between the layers of the underlying stack. These temperature requirements strongly depend on the materials involved, and the application thermal budget. For instance, deposition of high-K dielectric protective oxides on transparent substrates (e.g., foils, polymers) for flexible electronic devices, is restricted to temperatures below $200^{\circ} \mathrm{C}$, which allows the use of low melting temperature materials. ${ }^{58}$ Temperatures below $200^{\circ} \mathrm{C}$ are also required to avoid higher intermixing by silicide formation, occurring at both interfaces of the $\mathrm{Mo} / \mathrm{Si}$ multilayer mirrors, when growing the protective layer. ${ }^{54,59}$ Due to the thermal budget, high-efficiency silicon solar cells need passivation of both surfaces (front and rear), usually by oxide protective layers deposited at temperatures below $400^{\circ} \mathrm{C} .{ }^{45}$ 
5. Minimal stress. Protective layers, like most materials in the form of thin films, can exhibit high values of in-plane stress upon deposition. ${ }^{60}$ Thus, the protective layer must be able to be deposited such that a minimal stress is provided to the layered stack underneath.

6. Thermally stable. During the processing steps involved in the fabrication of electronics, ${ }^{22}$ photovoltaics, ${ }^{45}$ and also during their functionality (including the EUVL optics functionality), ${ }^{54,61}$ enhanced temperatures are achieved, where a thermally stable protective layer is required. For instance, in DRAMs and CMOS devices, the high-K dielectric protective layer should be thermodynamically stable on silicon upon annealing in oxidative environments. Apart from silicate formation, the possible crystallization of this layer during annealing can cause undesired current leakage through grain boundaries, and the formation of anisotropic crystalline phases can lead to nonuniformities in the dielectric constant. ${ }^{62,63}$ Similar concerns are also present in the fabrication of solar cells related to the photo-generated power, including the cyclical thermal loading caused by UV light in corrosive atmospheric conditions. ${ }^{45}$ Likewise, absorbed EUV radiation provides energy to the EUVL multilayer in the form of heat, which can reach elevated temperatures, depending on the mirror's geometrics and the cooling system. ${ }^{61}$ This means that the protective layer, which is part of the mirror, should be thermally stable together with the multilayer stack.

7. Compatible with environmental, health and safety regulations. A protective material should be a solid, not dangerous (toxic or radioactive) or highly reactive and unstable material under ambient conditions. Besides, it should not form hydroxides, which are hygroscopic and unstable..$^{40,45,64}$

8. Oxygen diffusion barrier. A protective material should have a dense structure to prevent inwards transport of oxygen (oxygen diffusion). ${ }^{54}$ Amorphous structures are usually preferred to polycrystalline, since grain boundaries are important paths for diffusion. ${ }^{34}$ However, regardless of the structure, a proper protective material should be free of defects (such as interstitial sites, dislocations or vacancies) that allow oxygen to diffuse. ${ }^{54}$

9. Resistant to reducing agents. In all discussed applications, the protective layer should not degrade under the effect of reducing agents such as hydrogen (gas or plasma) or similar agents, usually delivered at enhanced temperatures. For instance, a standard final anneal in the fabrication of CMOS devices is performed by a gas mixture (typically $90 \% \mathrm{~N}_{2}: 10 \% \mathrm{H}_{2}$ ), which is believed to passivate the interfacial traps (dangling bonds) with hydrogen. ${ }^{22}$ Since many high-K dielectric protective oxides might be reduced by this gas mixture, these protective layers should be carefully chosen to resist this treatment. Similarly, hydrogen plasma is used to passivate electron trap defects such dangling bonds, and to produce an additional doping in the bulk of the grain in Si- 
terminated solar cells. ${ }^{45,47,65}$ Even if this Si passivation effect can be achieved directly, for instance, by using a transparent conductive oxide (TCO) as protective layer, sometimes, the TCO-capped Si solar cell might be exposed to hydrogen plasma at enhanced temperatures to improve its electrical performance by annealing the $\mathrm{Si}$ material, while increasing the electrical conductivity of the TCO. ${ }^{65}$ Although, this is usually performed by TCOs functioning as contact electrodes (at the rear surface), these oxide protective layers should not be fully reduced by such hydrogen treatment. Correspondingly, a constant EUV-induced hydrogen plasma is normally generated inside the EUVL tool to remove external contaminants such as C or Sn from the mirrors' surface, ${ }^{37,66}$ which preserves their reflectance, enlarging their lifetime. ${ }^{36}$ Since these mirrors are capped by protective layers, ${ }^{54}$ these materials, especially oxides, should also not be reduced upon hydrogen exposure.

10. Resistant to UV light and EUV radiation. In the case of photon-based applications, the protective layers used in solar cells or EUVL optics should not degrade by either UV from sunlight or EUV radiation, which can result in a decrease of the respective cell efficiency or mirror reflectance. For instance, positive charges can be generated in a $\mathrm{SiN}_{\mathrm{x}}$ passivation layer, degrading the cell efficiency. ${ }^{67}$ Another example is the EUV-induced oxidation of a Ru-capped MLM, which leads to a loss of reflectance. ${ }^{49}$

11. Non-affine surface to external contaminants. A protective layer should provide a surface where external contaminants do not easily adhere by physical or chemical forces, and in case they adhere, they should be easily removable from its surface by cleaning agents. For instance, moisture is a critical contaminant during the fabrication of thin films. It can cause several problems such as change in adhesion properties, or particle and haze formation. Moisture also results in corrosion via oxidation of several components. In DRAMs and CMOS devices, it might diffuse through the high-K dielectric protective layers and interact with the underneath $\mathrm{Si}$, leading to $\mathrm{SiO}_{2}$ or silicate formation. ${ }^{68}$ Solar cells might also experience a strong moisture ingress, usually from the contact (or passivation) layer towards the center of the cell, affecting its overall conversion efficiency. ${ }^{69}$ Thus, both dielectric and contact (or passivation) layers should prevent this phenomenon from occurring. Another example is the contamination of EUVL mirrors by $\mathrm{C}$ or Sn nanoparticles, where the protective layers should be chosen to have a surface that mitigates contamination. ${ }^{36}$ In the case of Sn, it has been observed that not only the surface contributes to its adhesion on some metal surfaces, the bulk electronegativity difference between the protective material and $\mathrm{Sn}$ can also influence this property, and hence the cleaning process. As a first guide, Sn is only fully etched from materials with an electronegativity smaller than Sn. Moreover, there may be a larger amount of remaining Sn on the surface as the electronegativity of the material increases. ${ }^{37}$ 
12. Optimal thickness for a proper capacitance, UV transmission and EUV reflection. In CMOS and memory devices, there is an optimal thickness for the high-K dielectric protective layer that results in an aimed capacitance value, apart from the minimum thickness to close the layer, as mentioned before in this section. ${ }^{22}$ For both photon-based applications, apart from this minimum thickness, the oxidation resistant protective layers should have an optimal optical thickness to obtain either a maximum UV transmission (for solar cells) ${ }^{45}$ or EUV reflectance (for EUVL optics) ${ }^{36}$, when combined with the layered stack underneath. Basically, two main properties are required for the protective layer design to reach this optimal optical thickness: a low UV or EUV absorption of the protective material (low $\beta$ coefficient) and either, to have a minimal reflection (when acting as antireflection layer in solar cells), ${ }^{45}$ or not to reflect out of phase with the underneath EUV mirror. ${ }^{40}$ In this last application, not only the optimization of the optical thickness is important, but also the last layer of the multilayer (or diffusion barrier, if there is) can be optimized to get the minimal absorption (node) in the protective layer.

\subsubsection{Material selection criteria based on oxidation properties}

From the various requirements discussed in the previous section (Sec. 1.2.1), since this thesis is focused on the study of oxidation resistant layers for protecting DRAMs, CMOS devices, solar cells and EUVL optics against oxidation, material selection criteria based on oxidation (or oxygen diffusion) of (or through) the protective material are needed. Based on these criteria, a useful method to select different elements from the periodic table, according to their oxidation properties (oxide formation, oxide stability), is the use of an Ellingham diagram. ${ }^{11}$

An Ellingham diagram is a graphical representation of the thermodynamic driving force for a particular reaction to occur, across a temperature range under a certain pressure. ${ }^{70}$ This driving force depends on the sign of $\Delta G$, the Gibbs free energy change of reaction. Negative values for $\Delta G$ indicate that the reaction will proceed spontaneously without external energy input, while positive values indicate that the reaction will require external energy to proceed. The temperature dependency of this thermodynamic quantity under a constant pressure is described by

$$
\Delta G=\Delta H-T \Delta S,^{71}
$$

where $\Delta H$ is the enthalpy change of the reaction, $\Delta S$ is the entropy change, and $T$ is the absolute temperature in $\mathrm{K} . \Delta H$ is a measure of the liberated energy when the reaction occurs (heat of reaction). If the reaction releases energy, $\Delta H$ is negative (exothermic process), while if the reaction requires energy, $\Delta H$ is positive (endothermic process). $\Delta S$ is a measure of the disorder of the products compared to the reactants. For instance, if a gas (a highly disordered state) reacts with a liquid (a less disordered state) to form a solid 
(an ordered state), there is an increase of the order of the system, which usually translates in a negative value of $\Delta S$.

A typical Ellingham diagram plots the standard Gibbs free energy (change) of formation $\Delta G_{f}^{\circ}$ of various oxides (oxidation reaction) as function of temperature $T$. Usually each oxidation reaction is normalized to consume $1 \mathrm{~mol} \mathrm{O}_{2} \cdot{ }^{70}$ However, in our case, we have normalized each reaction to form 1 mol oxide $M_{x} O_{y}$, since it is more useful for the direct comparison between all oxides according to their stability once they are formed, following the reaction:

$$
x M+\frac{y}{2} O_{2} \rightarrow M_{x} O_{y}
$$

where $x$ and $y / 2$ are the stoichiometric coefficients of the respective element $M$ and oxygen $\mathrm{O}_{2}$ in the reaction (see Fig. 1.1). Both standard enthalpy and entropy of formation $\left(\Delta H_{f}^{\circ}\right.$ and $\Delta S_{f}^{\circ}$ ) of oxides do not change significantly with temperature as long as there is no change of phase or state, as observed experimentally by Ellingham. ${ }^{11}$ Thus, the plot of $\Delta G_{f}{ }^{\circ}$ vs. $T$ leads to straight lines for each oxidation reaction with the form of:

$$
\Delta G_{f}^{o}=\Delta H_{f}^{o}-T \Delta S_{f}^{o, 71}
$$

where the intercept of these lines at $0 \mathrm{~K}$ gives $\Delta H_{f}^{\circ}$ and their slope gives $-\Delta S_{f}^{\circ}$ (see lines in Fig. 1.1). The formation of most oxides usually exhibits negative values for $\Delta H_{f}^{\circ}$, since it is an exothermic process (as seen for all reactions of formation displayed in Fig. 1.1, except for $\mathrm{Au}_{2} \mathrm{O}_{3}$ formation, which is an endothermic process). In the temperature range normally used, the element and its oxide are in condensed state (solid or liquid), and oxygen is a gas with larger entropy compared to these condensed materials, such that $\Delta S_{f}^{\circ}$ is negative for most of oxidation reactions, leading to a positive slope $-\Delta S_{f}^{\circ}$ (as shown for all depicted oxides in Fig. 1.1, except for $\mathrm{RhO}_{2}$ and $\mathrm{PtO}_{2}$ that are gases). Note that the standard enthalpy of formation $\Delta H_{f}^{\circ}$ is a measure of the stability of the compound, since the reverse of the formation reaction describes how readily the compound decomposes into its elements. ${ }^{72}$ Therefore, more negative values of $\Delta H_{f}{ }^{\circ}$ for the reaction of oxide formation would signify more energy required to decompose the oxide and, therefore, higher oxide stability. In the case of a typical Ellingham diagram, $\Delta H_{f}{ }^{\circ}$ cannot be directly obtained by the intercept of the $\Delta G_{f}{ }^{\circ}-T$ lines at $0 \mathrm{~K}$ and it should be corrected by the stoichiometric coefficient of each oxidation reaction. In contrast, in our case, $\Delta H_{f}^{\circ}$ is obtained by the direct intercept of the lines at $0 \mathrm{~K}$. The consumption of $1 \mathrm{~mol} \mathrm{O}_{2}$ for a common Ellingham diagram leads to almost parallel lines for the oxidation reactions. ${ }^{11}$ However, the lines in our modified Ellingham diagram are only parallel for the formation of oxides with similar stoichiometry, that consume the same amount of oxygen, and thus have similar entropy change (see lines in Fig. 1.1). 
The relative position of the lines in the y-axis $\left(\Delta G_{f}^{\circ}\right)$ indicates the spontaneity of the formation of oxides in the temperature range ( 1 mol oxide in our case), since $\Delta G_{f}{ }^{\circ}$ is negative for most of oxides (Fig. 1.1). Thus, elements on the top of the diagram such as most of noble metals ( $\mathrm{Ru}, \mathrm{Rh}, \mathrm{Pd}, \mathrm{Ag}$, Ir, $\mathrm{Pt}$ and $\mathrm{Au}$ ) are low-oxidation materials that usually form unstable oxides, which are easily reduced to their elemental state (for instance, by hydrogen plasma). In the middle of the diagram there are elements such as $\mathrm{Co}, \mathrm{Zn}, \mathrm{Ni}, \mathrm{Mo}$, etc., that present higher affinity for oxygen, forming oxides of greater stability. Finally, at the lower part of the diagram, there are elements such as Zr, Ti, Hf, $\mathrm{Al}, \mathrm{Y}, \mathrm{Ta}$, etc., that strongly oxidize forming very stable oxides, the so-called refractory oxides, which are very difficult to reduce. ${ }^{70}$

Fig. 1.1 shows the modified Ellingham diagram according to the reaction Eq. (1.2) for various elements of the periodic table. Note that only solid, stable (not hygroscopic or highly reactive at ambient conditions), and non-dangerous (toxic or radioactive) elements are considered for the diagram. Thus, alkalis, alkaline- and rare-earth metals, gases, liquids and toxic elements are not included in the diagram.

Based on the previous description, the modified Ellingham diagram directly provides two main characteristics about oxidation of materials: spontaneity of an element to form an oxide $\left(\Delta G_{f}^{\circ}\right)$, and the relative stability of the oxides once they are formed $\left(\Delta H_{f}^{\circ}\right)$. This allows selecting elements or their oxides, according to their oxidation (for elements) or oxygen diffusion (for oxides) properties, as proper oxidation resistant protective layers. Apart from elements and oxides, it is possible to consider other compounds such as carbides, nitrides or silicides as protective layers for Si-terminated electronic, photovoltaic and optical devices. The problem is that these compounds usually oxidize in air, even partially, forming oxycarbides, oxynitrides or silicates. ${ }^{40}$ Therefore, only low-oxidation elements and stable oxides are considered as protective layers against oxidation/oxygen diffusion. Looking at the diagram these materials correspond to the top and the bottom materials of the graph, respectively (see Fig. 1.1).

From the top of the diagram, low-oxidation elements that form unstable oxides, easily reduced by hydrogen such as noble metals (in order of decreasing $\Delta G_{f}^{\circ}$ at $25^{\circ} \mathrm{C}$ : $\mathrm{Au}, \mathrm{Ag}, \mathrm{Pd}, \mathrm{Ir}, \mathrm{Pt}, \mathrm{Ru}$ and $\mathrm{Rh}$ ) can be considered as protective materials (Fig. 1.1). $\mathrm{Cu}$ also should be part of these elements according to $\Delta G_{f}^{\circ}$, but the problem is that it forms a relatively thick $\left(\sim 3-5 \mathrm{~nm} \mathrm{CuO} / \mathrm{Cu}_{2} \mathrm{O}\right)$ passivation layer upon air exposure ${ }^{73}$ compared to the other noble metals which do not oxidize $(\mathrm{Au}, \mathrm{Ag}, \mathrm{Pd}, \mathrm{Pt}),{ }^{74}$ or form few oxide monolayers of chemically adsorbed oxide $(<1 \mathrm{~nm})$ at room temperature ( $\mathrm{Ir}, \mathrm{Ru}, \mathrm{Rh}){ }^{75,76}$

From the bottom of the diagram, stable oxides that do not allow oxygen to penetrate through them and oxidize the top Si layer from the device, can also be considered as protective layers. As discussed previously, there is an increase of the oxide stability with the decrease of $\Delta H_{f}^{\circ}$. However, only oxides with lower $\Delta H_{f}^{\circ}$ than $\mathrm{SiO}_{2}$ might be 
considered, since the $\mathrm{Si}$ layer slightly oxidizes $\left(\mathrm{SiO}_{\mathrm{x}}\right.$ formation, with $\left.0 \leq \mathrm{x}<2\right)$ upon oxide deposition. Thus, oxides with higher stability than $\mathrm{SiO}_{2}$ will not thermodynamically decompose and transfer the oxygen to the oxidized Si layer. These oxides range from $\mathrm{In}_{2} \mathrm{O}_{3}$ to $\mathrm{Ta}_{2} \mathrm{O}_{5}$ in order of decreasing $\Delta H_{f}^{\circ}$ (see Fig. 1.1). However, not all these oxides are stable until $400^{\circ} \mathrm{C}$ (the typical maximum temperature for all considered applications). ${ }^{22,47,61} \mathrm{Sb}_{2} \mathrm{O}_{5}$ decomposes at about $300^{\circ} \mathrm{C},{ }^{77}$ just before melting at $380^{\circ} \mathrm{C}$ (see $d$ in Fig. 1.1), $\mathrm{V}_{2} \mathrm{O}_{3}$ slowly converts to $\mathrm{V}_{2} \mathrm{O}_{4}$ upon air exposure, ${ }^{78} \mathrm{Re}_{2} \mathrm{O}_{7}$ sublimates at $250^{\circ} \mathrm{C}$ (see $s$ in Fig. 1.1), $\mathrm{V}_{2} \mathrm{O}_{4}$ changes phase (from $\alpha$ to $\beta$ ) at $\sim 71^{\circ} \mathrm{C}$ (see change of slope in Fig. 1.1), and $\mathrm{V}_{2} \mathrm{O}_{5}$ decomposes upon annealing in vacuum at temperatures below $400^{\circ} \mathrm{C} .{ }^{79,80}$ This reduces the number of possible candidate materials as protective layers for DRAMs, CMOS devices, solar cells, and EUVL optics.

It should be noted that the choice of material using the modified Ellingham diagram is based on thermodynamics which usually are favorable at high temperatures, but low temperature oxide formation strongly depends on kinetics. ${ }^{81}$ Although kinetics are ignored in the formation and stability (decomposition) of the oxides, the use of this diagram seems a first feasible method to discriminate elements or oxides according to their oxidation properties (for elements) and oxide stability (for oxides).

As explained previously in Sec. 1.2.1, apart from preventing oxidation of the underlying layers, there are other properties such as the optimal thickness (for a proper capacitance, UV transmission and EUV reflectance), the resistance to reducing agents (hydrogen), or the easy-to-clean/sticking resistance to pollutants (moisture, C, Sn, etc.), which need to be taken into account for choosing a material as proper protective layer for DRAMs, CMOS devices, solar cells, and EUVL optics. However, they are not the scope of this thesis which is based on the study of relevant (oxidation) resistant protective layers. In particular, this thesis will be focused on the study of $\mathrm{Ru}$ and $\mathrm{ZrO}_{2}$ oxidation resistant protective layers. Ru has been chosen, apart from its low-oxidation properties, ${ }^{11}$ because it fulfils most of the other requirements, and has been used in several application such as $\mathrm{Cu}$ diffusion barrier and/or $\mathrm{Cu}$ seed layer in integrated circuits with copper interconnect technology, ${ }^{4}$ as bottom electrode in capacitors based on high dielectric materials, ${ }^{5,6}$ or as reference capping material for protecting EUVL optics against oxidation. ${ }^{7,40} \mathrm{ZrO}_{2}$ has also been selected in this thesis as alternative oxidation resistant protective material for similar applications ${ }^{22,34,41}$ (including photovoltaics) ${ }^{39}$ mainly due to its low reactivity with $\mathrm{Si}^{32}$ together with the fact that $\mathrm{Zr}$ forms a very stable and inert oxide to possible external contaminants. ${ }^{36,37,38}$ In the next section, the current status of oxidation resistant capping layers designed for protecting microelectronic (DRAM and CMOS) devices, solar cells and EUVL optics against oxidation, will be discussed, including $\mathrm{Ru}$ and $\mathrm{ZrO}_{2}$ protective layers. 


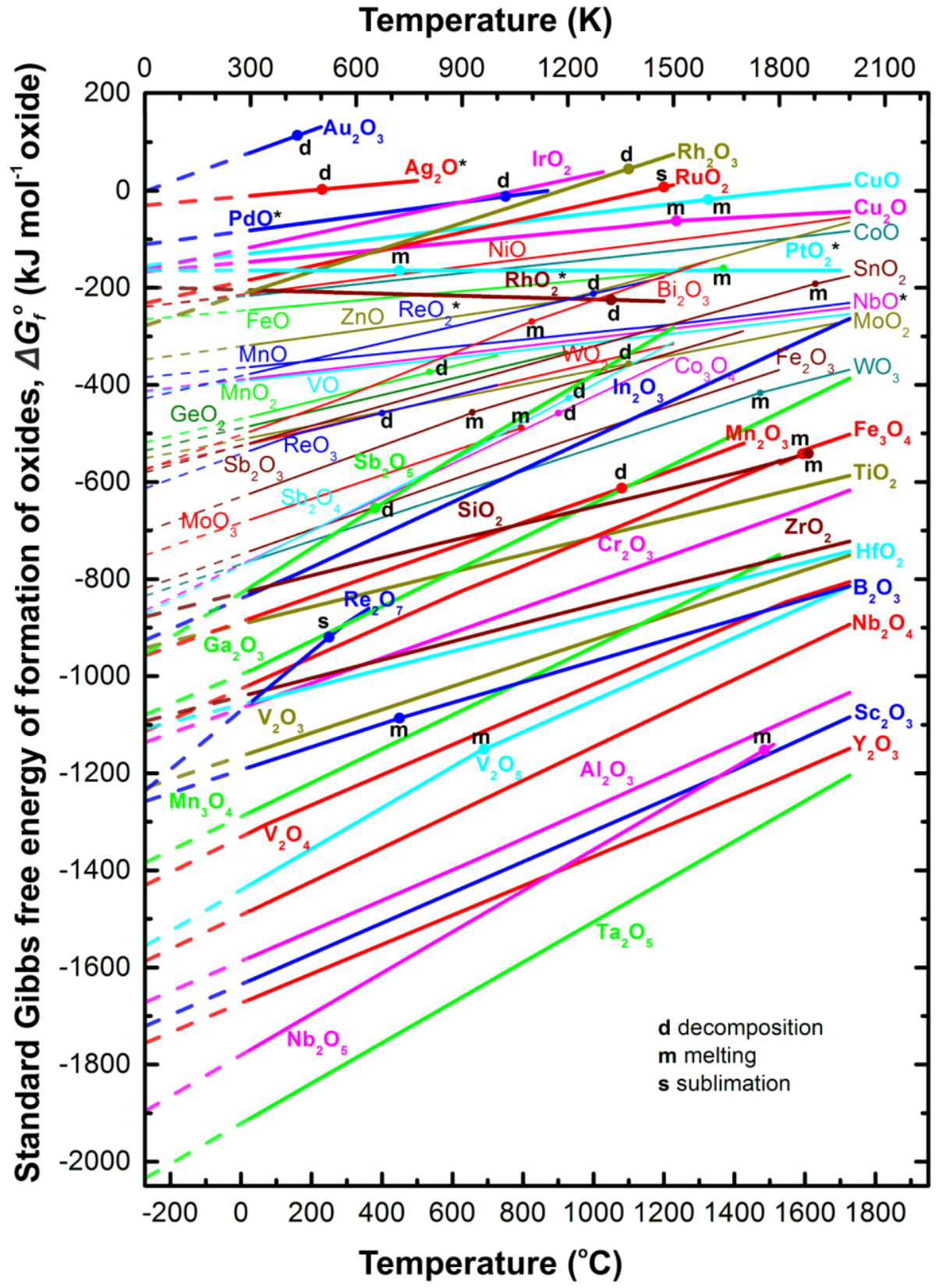

Fig. 1.1. Modified Ellingham diagram for various elements of the periodic table. Solid lines show the thermodynamic data available within the temperature range according to Eq. (1.3) for each reaction of oxide formation ( $1 \mathrm{~mol}$ oxide formed). Dashed lines are extrapolation of this data from $25^{\circ} \mathrm{C}$ to $0 \mathrm{~K}$. The decomposition $d$, melting $m$ and sublimation $s$ points are pointed out. ${ }^{38}$ Thermodynamic data for $\Delta G_{f}^{\circ}$ of formation of oxides with * is from ref. ${ }^{82}$. The rest of the data is from ref. ${ }^{83}$. 


\subsubsection{Current status}

\subsubsection{Microelectronic (DRAM and CMOS) devices}

There are several studies using different capping materials for protecting gate electrodes against oxygen diffusion for MOS-like devices. For instance, Ru and $\mathrm{W}$ capping materials have been tested upon thermal oxidation, and subsequent vacuum annealing (thermal stability) to understand their strength in protecting MoTa metal gate electrodes against oxidation, when applied on $\mathrm{SiO}_{2}$ gate dielectrics. ${ }^{84}$ Another example of a thermal oxidation study, following both thermal stability and oxygen diffusion barrier properties, has been the one carried out for the $\mathrm{RuO}_{2} / \mathrm{Ru}$ bilayer structure, used as bottom electrode structure for capacitors with a high dielectric constant. ${ }^{85}$ In this bilayer, Ru has been applied as a sacrificial layer, preventing inwards oxygen diffusion into underlying layers of the electrode. The same bilayer structure has already been proposed and investigated within a $\mathrm{RuO}_{2} / \mathrm{Ru} / \mathrm{TiN} / \mathrm{TiSi}_{\times} /$poly-Si storage node ${ }^{86}$ Oxygen transport studies have also been reported for Pt and Re protective layers, considered also as bottom gate electrodes for MOS-like devices. ${ }^{87,88,89}$ In the case of gate dielectric layers for CMOS and memory (DRAM) devices, several (usually high-K) binary oxides such as $\mathrm{Ta}_{2} \mathrm{O}_{5},{ }^{90,91} \mathrm{Y}_{2} \mathrm{O}_{3},{ }^{92,93} \mathrm{Al}_{2} \mathrm{O}_{3},{ }^{91,94,95,96} \mathrm{HfO}_{2},{ }^{97,98,99,100} \mathrm{ZrO}_{2},{ }^{34,101,102,103}$ and $\mathrm{TiO}_{2},{ }^{104,105,106}$ and also perovskite materials such as $\mathrm{SrTiO}_{3}$ and $(\mathrm{Ba}, \mathrm{Sr}) \mathrm{TiO}_{3}$ have been investigated, ${ }^{107,108}$ according to their electrical and/or other physical properties after/or during growth. Apart from the characterization of the grown layers, in some of these oxide studies, oxygen diffusion through the gate dielectric towards deeper layers has also been investigated. ${ }^{91,96,98,99,100,102,103}$ In addition, the thermodynamic stability in contact with Si of most of the mentioned binary oxides, has been evaluated..$^{32}$ All presented oxide materials have been considered as a replacement of the typical $\mathrm{SiO}_{2}$ gate dielectric layer, which exhibits high gate leakage current at small thicknesses $(<1 \mathrm{~nm})$, required for the continuous downscaling of microelectronics. ${ }^{22,109}$

\subsubsection{Solar cells}

Several materials, especially oxides, have been considered for the passivation of both surfaces (front and rear) of high-efficiency silicon solar cells. ${ }^{47}$ These surface passivation layers are not only used to prevent degradation of photovoltaics by atmospheric corrosion and cyclical thermal loading during sunlight, but also for antireflection purposes and, to provide electronic passivation by minimizing the recombination losses at both surfaces (or interfaces), reducing the amount of interfacial defects upon their application. There are several studies on different passivation layers attending to various physical properties, including photo efficiency, electrical transport, antireflection and oxidation. For instance, the surface recombination velocity has been investigated for $\mathrm{SiN}_{\mathrm{x}}$, as outstanding front passivation layer for crystalline $\mathrm{Si}(\mathrm{c}-\mathrm{Si})$ solar 
cells. ${ }^{110}$ Thermally grown $\mathrm{SiO}_{2}$ and atmospheric chemical vapor deposited $\mathrm{TiO}_{2}$ have also been studied as possible front passivation layers. However, the high thermal budget required for the fabrication of $\mathrm{SiO}_{2}$ together with its low refractive index, and the poor surface electronic passivation of $\mathrm{TiO}_{2}$, have make them not to be considered for the upcoming industrial production of c-Si photovoltaics. ${ }^{47}$ Amorphous $\mathrm{Al}_{2} \mathrm{O}_{3}\left(\mathrm{a}-\mathrm{Al}_{2} \mathrm{O}_{3}\right)$ has also been investigated as excellent passivation layer, with advantages over the traditional $\mathrm{SiN}_{\mathrm{x}}$ passivation layer for industrial solar cell production. ${ }^{47,111,112}$ Amorphous $\mathrm{Al}_{2} \mathrm{O}_{3}$ has been applied on both front and rear surfaces in c-Si photovoltaics, giving low interfacial defect density together with a strong field-effect passivation induced by a negative fixed charge density. ${ }^{111,112}$ Thin a- $\mathrm{Al}_{2} \mathrm{O}_{3}$ layers have also been applied as transparent conductive oxide (TCO) barriers in dye-sensitized solar cells (DSSCs), for instance, for protecting $\mathrm{TiO}_{2}$ nanoparticles coated with dye molecules immersed under an electrolyte solution. ${ }^{113}$ Several studies have been reported to investigate this $\mathrm{Al}_{2} \mathrm{O}_{3}-\mathrm{TiO}_{2}$ system, for improving the performance of this type of solar cells. ${ }^{14,115,116,117} \mathrm{~A}$ thin $\mathrm{TiO}_{2}$ layer has also been studied as TCO, instead of $\mathrm{Al}_{2} \mathrm{O}_{3}$ for similar DSSCs. ${ }^{118}$ Other oxide layers such as $\mathrm{HfO}_{2}$ and $\mathrm{ZrO}_{2}$ have also been considered and studied as TCOs for DSSCs. ${ }^{116,119}$ Due to the high sensitivity of organic materials such as polymers, organic photovoltaic (OPV) devices and organic light-emitting diodes (OLEDs) are normally encapsulated by a thin protective oxide layer which protects them from oxidative species such as water and oxygen. ${ }^{120,121,122}$ Various oxide layers such as $\mathrm{In}_{2} \mathrm{O}_{3}, \mathrm{SnO}_{2}, \mathrm{ZnO}$, and their mixtures, ${ }^{123}$ and other oxide thin films including $\mathrm{Al}_{2} \mathrm{O}_{3}{ }^{124}$ or $\mathrm{ZrO}_{2},{ }^{125}$ and their combination in the form of $\mathrm{Al}_{2} \mathrm{O}_{3} / \mathrm{ZrO}_{2}$ multilayer structures, ${ }^{126,127}$ have been adopted as TCOs and studied for the protection of such organic devices.

\subsubsection{EUVL optics}

Simulations of EUV reflectance have been performed for several elements $(Z=1$ 92) and for various compounds such as oxides, nitrides, carbides or silicides (for which the optical constants exist) for different thicknesses $(2-4 \mathrm{~nm})$, as oxidation resistant protective layers on top of either Mo- or Si-terminated Mo/Si multilayer mirrors. ${ }^{40}$ Apart from EUV reflectance simulations, there are several reported studies where the EUV reflectance is experimentally measured, before and after EUV exposure, (or similar ebeam exposure) in a water vapor environment from Si-terminated Mo/Si MLMs, capped with different protective layers (with or without diffusion barriers) such as $\mathrm{Ru},{ }^{128}$ $\mathrm{Ru} / \mathrm{Mo},{ }^{128,129} \mathrm{Ru} / \mathrm{B}_{4} \mathrm{C},{ }^{49,128} \mathrm{Pt} / \mathrm{Mo},{ }^{129} \mathrm{SiO}_{2},{ }^{41,130} \mathrm{TiO}_{2},{ }^{130,131} \mathrm{RuO}_{2},{ }^{130,131} \mathrm{ZrO}_{2},{ }^{41,130}$ $\mathrm{V}_{2} \mathrm{O}_{5},{ }^{130} \mathrm{Cr}_{2} \mathrm{O}_{3},{ }^{130} \mathrm{Mn}_{2} \mathrm{O}_{3},{ }^{130} \mathrm{Y}_{2} \mathrm{O}_{3},{ }^{130} \mathrm{Nb}_{2} \mathrm{O}_{5},{ }^{130} \mathrm{Rh}_{2} \mathrm{O}_{3},{ }^{130} \mathrm{PdO},{ }^{130} \mathrm{SnO}_{2},{ }^{130} \mathrm{La}_{2} \mathrm{O}_{3},{ }^{130}$ $\mathrm{CeO}_{2},{ }^{130} \mathrm{WO}_{3} .{ }^{130}$ Most of these studies not only measure the EUV reflectance upon exposure, but also investigate the EUV-induced oxidation resistance of the protective material (and/or underlying multilayer). ${ }^{41,49,128,131}$ In this line, there are other studies reported that test different protective materials against oxidation on a multilayer (or a single Mo or Si layer, mimicking the last layer of the multilayer), not only by EUV (or 
e-beam) exposure in presence of water, ${ }^{132}$ but also by atomic oxygen, ${ }^{133}$ by oxygen plasma, ${ }^{134}$ or by ambient air. ${ }^{8}$ In addition, several of these studies give some insights on the effect of the growth (thickness, ${ }^{132}$ conditions $^{8}$ ) on the oxidation resistance of the deposited protective layers.

When considering the current status of oxidation resistant protective layers for EUVL optics, electronics and photovoltaics presented in this and the previous two sections (Secs. 1.2.3.3, 1.2.3.1 and 1.2.3.2, respectively), the processes involved in the growth and the oxidation of these protective layers, including their oxidation (or oxygen diffusion) kinetics, are not well understood, and that is the focus of this thesis. In particular, this thesis presents a detailed study of the initial growth until layer closure (processes, phenomena) and oxidation (mechanisms, diffusion kinetics) of $\mathrm{Ru}$ and $\mathrm{ZrO}_{2}$ thin films used as oxidation resistant protective layers for DRAMs, CMOS devices, solar cells (only $\mathrm{ZrO}_{2}$ ), and EUVL optics. In the next two sections, thin film growth (Sec. 1.3) and oxidation (Sec. 1.4), will be discussed in more detail, including $\mathrm{Ru}$ and $\mathrm{ZrO}_{2}$ thin films.

\subsection{Growth of thin films}

\subsubsection{Choice of deposition technique}

Thin films can be deposited by several techniques that are usually classified in two types: chemical and physical processes, depending on the principle causing thin film deposition, as described as follows.

Chemical deposition uses a fluid (liquid or gas) precursor which undergoes a chemical reaction at a solid target surface, producing a thin solid film. Since the fluid surrounds the target, deposition occurs equally in each surface, regardless of the direction (conformal deposition). Chemical deposition includes processes such as electroplating, ${ }^{111}$ sol-gel, ${ }^{47}$ spin coating, ${ }^{36}$ or chemical vapor deposition (CVD). ${ }^{135}$

Physical deposition uses a physical process (mechanical, electromechanical or thermodynamic) to produce a thin solid film. Physical deposition processes are usually performed at low pressures (usually vacuum), and are also referred to as physical vapor deposition (PVD). The material to be deposited (target) is located in an energetic environment, where its particles can easily escape from its surface. In front of this target, there is a cooler surface (substrate) that draws energy of these particles upon arrival, forming a thin solid film. Since these particles follow a straight path under vacuum, physical deposition processes are normally directional rather than conformal. ${ }^{135}$ Physical deposition includes processes such as thermal evaporation, ${ }^{48,113}$ pulsed laser deposition (PLD) ${ }^{44}$ cathodic arc deposition (Arc-PVD), ${ }^{135}$ electrospray deposition, ${ }^{46}$ e-beam evaporation, ${ }^{136}$ or magnetron sputtering. ${ }^{137}$ 
Chemical deposition techniques are usually not adequate because reactions lead to formation of side products or unreacted precursors, especially in solution, which are not easily cleaned and might induce impurities on the grown films. ${ }^{135}$ In the case of "ordinary" CVD, all reacting gases are brought together into the reaction chamber, leading to a high growth rate, but with the possibility of uncontrolled growth and other side reactions. Within the CVD processes, there exists a more controlled mode of operation for ultra-thin film deposition in a layer-by-layer fashion, which is normally referred as atomic layer deposition (ALD) or as atomic layer CVD (AL-CVD). AL-CVD differs from the "ordinary" CVD by the fact that uses sequential, self-limiting surface catalytic reactions of usually two gas phase chemical precursors, pulsed separately into the reaction chamber, followed by a flushing step using inert gas. ${ }^{138}$ Although sometimes AL-CVD, or even "ordinary" CVD, can be extremely clean and are often applied in microelectronics (DRAMs and CMOS devices), ${ }^{22}$ or even in photovoltaics, ${ }^{113}$ the use of high temperatures (typically between $\left.200-1600^{\circ} \mathrm{C}\right)^{139}$ might affect the intermixing (between the protective material and the layer underneath or between the layers of the underlying stack), the morphology and the growth mode of the deposited films. For instance, in the study performed by Shin et al., ${ }^{140}$ low-temperature CVD Ru was observed to grow columnar in a $3 \mathrm{D}$ growth mode at temperatures ranging from 150 to $250^{\circ} \mathrm{C}$, while low-temperature $\left(\sim 25^{\circ} \mathrm{C}\right)$ DC magnetron sputtered $\mathrm{Ru}$ was growing smoothly in a kind of layer-by-layer (or 2D) growth mode. It should be noted that different conditions could also enhance morphology changes for DC sputtered Ru films (for instance, higher sputter gas pressures can induce columnar growth in $\mathrm{Ru}$ ), ${ }^{141}$ but in the study by Shin et al., ${ }^{140}$ the conditions were chosen such that smooth Ru films $(\sim 0.11$ nm RMS roughness) were obtained using DC sputtering. However, even though several CVD studies have produced smoother Ru films, ${ }^{142,143,144}$ the RMS roughness was still larger in most cases than by DC sputtering, and the substrate temperature during deposition could not be lower than $\sim 150^{\circ} \mathrm{C}$ for $\mathrm{Ru}$ nucleation, which is often problematic for flexible electronic devices such as OPV or OLEDs, due to the possible degradation of their organic substrates, ${ }^{120,121,122}$ or for EUVL optics, due to Mo/Si intermixing. ${ }^{145}$ In addition, for this last application, since EUVL optics consist of large, heavily curved mirrors, there is a necessity of lateral thickness profiles, which cannot be achieved by chemical deposition techniques that produce conformal coatings, and only physical methods such as PVD can provide a thickness gradient along the mirror surface, by means of masking or velocity profiles. ${ }^{7}$

In the case of physical deposition techniques, thermal evaporation is mostly suitable for low melting point target materials, and the deposition rate is not easily controllable and reproducible. ${ }^{113}$ PLD is difficult to implement for coating large areas, ${ }^{44}$ such that its application is normally restricted to small target sizes. Electrospray deposition cannot be applied for inorganic materials with relatively high melting point ( $\mathrm{Mo}, \mathrm{Si}, \mathrm{Ru}$, etc.), since they should be in solution prior to their deposition. ${ }^{46}$ E-beam evaporation might be a 
proper option together with magnetron sputtering for deposition of oxidation resistant protective layers, and other underneath layers, in DRAMs, CMOS devices, solar cells and EUVL optics. Using e-beam evaporation, an additional ion treatment, either ion assistance and/or post deposition polishing, might be needed to get denser layers, which in the case of sputtering is not usually required. ${ }^{146}$ Only in some cases, the layers produced by sputtering are subject to an extra ion/plasma treatment, similar to e-beam (for instance, polishing each Si layer in the EUV multilayer by $\mathrm{Kr}^{+}$ions). ${ }^{147}$

From all mentioned deposition techniques, low-temperature (usually roomtemperature) magnetron sputtering is frequently used for thin film deposition of oxidation protective layers, as both gate electrodes and dielectric layers for CMOS and memory devices, ${ }^{22,34,85,148,149}$ as surface passivation and contact TCO layers for solar cells, ${ }^{45,111}$ or as capping layers for EUVL optics, ${ }^{7,36,40}$ and has been adopted in this thesis to grow both $\mathrm{Ru}$ and $\mathrm{ZrO}_{2}$ protective layers, including the underlying $\mathrm{Si}$ and barrier layers. In the next section, this technique will be discussed in more detail.

\subsubsection{Room-temperature magnetron sputtering}

\subsubsection{Principles and setup}

A typical magnetron sputtering setup (scheme in Fig. 1.2) consists of a substrate (usually grounded) placed at the top. A target material placed at the bottom and a shield surrounding the target are connected to a power supply, acting as the respective cathode and anode (also this last normally grounded). Both electrodes are enclosed in a high vacuum chamber filled with a noble gas such as Ar. Upon the application of power, this noble gas is ionized by energetic electrons emitted by the cathode, which leads to the formation of a plasma, confined close to the target surface by means of several magnets (see Fig. 1.2). The positive gas ions from this plasma are accelerated towards the target cathode due to Coulomb attraction. When these incident ions have an energy greater than the surface binding energy of the target material, target atoms will be ejected (or sputtered) from its surface, producing collision cascades between other sputtered and reflected particles from the target. The sputtered atoms present a wide energy distribution (up to tens of $\mathrm{eV}$ ) and are ejected in different directions. However, only when the energy of these atoms is almost thermal (about few eV or even below) due to several collisions with the sputtering gas (sometimes insignificant), they will diffuse randomly towards the walls of the deposition chamber or the substrate where they will condense, forming a thin coating.

The most common power supplies used in magnetron sputtering are direct current (DC) or radio frequency (RF). DC requires electrically conductive target materials, since during deposition of non-conductive materials e.g. dielectric films, the electrodes will be coated by insulating material, which will charge up and terminate the discharge. RF can 
be used for all target materials, since the positive charges accumulated during one halfcycle can be neutralized by electron bombardment during the next cycle. Although RF can be used for all materials, if the material exhibits some conduction (for instance, thin metal-oxides), DC is preferred over RF, due to its higher scalability and simplicity of the DC process. ${ }^{137}$

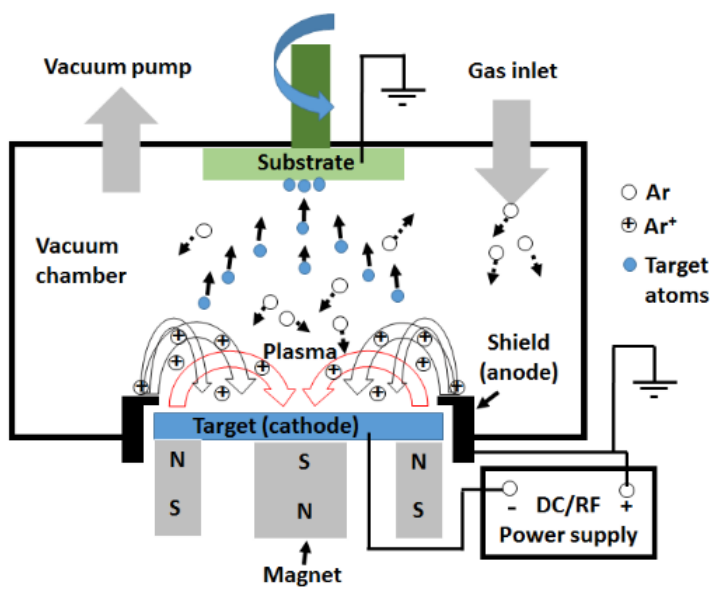

Fig. 1.2. Basic scheme of magnetron sputtering deposition. Gas $\mathrm{Ar}^{+}$ions sputter the target material which condenses at the substrate (or at the chamber walls), forming a thin coating. Note that the substrate in this scheme is rotated to ensure film uniformity, and it is also grounded (not biased).

There are several deposition parameters that can influence the growth process in room-temperature magnetron sputtering, by changing the properties of the deposited films (density, roughness, crystallinity, etc.) which result into different physical and chemical processes happening at the substrate surface during deposition. The main deposition parameters include: target-substrate distance and target-substrate angle, ${ }^{150,151}$ sputtering power, ${ }^{152}$ substrate bias, ${ }^{153}$ target self-bias, ${ }^{154}$ and sputter gas (type, pressure, and mixture composition for reactive sputtering). ${ }^{155,156,157}$ From all these parameters, the relevant ones, which are varied in this thesis are: the sputtering power, and both sputter gas pressure and mixture composition (in the case of reactive sputtering). These three relevant deposition parameters are discussed as follows.

\subsubsection{Relevant deposition parameters}

Sputtering power (or current/voltage for DC). In the case of DC, always one parameter (power, current, or voltage) of the sputter gun should be specified for controlling the sputtering process. ${ }^{152}$ Since both current and voltage control modes may lead to small drifts in the other parameters, leading to drifts in deposition rate, the power control mode is usually preferred because, drifts in deposition rate caused by drifts in voltage, are approximately compensated by drifts in current. Focusing on the sputtering 
power, it has a direct influence on the deposition rate. Higher power signifies higher sputter rate, due to higher current and/or higher voltage. This increase of power can strongly change the structure and morphology of the deposited films such as an increase of roughness or a change of crystal orientation. ${ }^{158,159,160}$ In this thesis, for each deposition setup used, a fixed power was chosen per material, in order to have a reasonable deposition rate between $\sim 0.1$ to $0.4 \AA \cdot \mathrm{s}^{-1}$.

Sputter gas pressure and mixture composition. The sputter gas pressure is a regulator of the particle kinetic energy, becoming slower and more diffusive for higher pressures due to higher scattering, which leads to increasing amount of thermalized (random) sputtered particles and a reduction of the ballistic (straight trajectory) flux. ${ }^{150,156}$ The effect of the gas pressure can significantly influence the structure and the morphology of the deposited films. ${ }^{141,161}$ Therefore, this parameter has been adapted in all chapters of this thesis, together with the sputtering power, to control the deposition rate and the energy distribution profile of the incoming material flux, and hence controlling the final properties such as the mass density or roughness of our grown films.

In the case of reactive sputtering, a target material is sputtered with a mixture of a noble gas (such as Ar) and a reactive gas (e.g., $\mathrm{O}_{2}$ or $\mathrm{N}_{2}$ ), usually leading to the formation of an oxide or a nitride of the target material. The composition of this mixture (noble gas + reactive gas) can be controlled by varying the partial pressure (or partial flow) of both components, normally expressed as gas concentration. The gas concentration also can influence both deposition parameters (e.g., deposition rate) and structural properties of the produced films (e.g., stoichiometry, density). For instance, Venkarataj et al. ${ }^{157}$ observed the influence of the $\mathrm{O}_{2}$ partial pressure on the deposition of $\mathrm{ZrO}_{2}$ by DC magnetron sputtering in a $\left(\mathrm{O}_{2}+\mathrm{Ar}\right)$ mixture. There was a clear transition from an oxidic sputtering mode (for high oxygen content) to a metallic sputtering mode (for low oxygen content), which resulted in different properties of the produced films. The oxidic sputtering mode exhibited a lower sputtering rate, due to poisoning of the target by oxygen. However, the stoichiometry of such films was close to $\mathrm{ZrO}_{2}$, while a substoichiometric oxide was obtained for low oxygen content, due to less oxygen in the mixture. The oxide was denser for the metallic mode, close to the $\mathrm{Zr}$ density. In contrast, for the oxidic mode, the oxide had about $90 \%$ of the density of bulk $\mathrm{ZrO}_{2}$. Similar sputtering modes have been selected in this thesis (Chapter 6) for growing the $\mathrm{ZrO}_{2}$ thin films, and their influence in the structural properties such as stoichiometry and density has also been studied.

There are several (physical and chemical) phenomena happening at the substrate surface during thin film deposition such as sputtering induced intermixing, ${ }^{162}$ nucleation and growth, ${ }^{163,164}$ compound formation, ${ }^{72}$ and surface segregation. ${ }^{165}$ From all these phenomena, the relevant ones for this thesis are discussed as follows. 


\subsubsection{Relevant processes occurring during deposition}

Sputtering induced intermixing. The impact of sputter gas ions (or neutrals) scattered from the target can induce intermixing on the growing film, due to surface resputtering and ion implantation. ${ }^{162}$ The amount of particles scattered from a material target and their energy distribution can be estimated by using a simulation software such as SRIM (or TRIM). ${ }^{166}$ These values depend on the amount, type, energy and angle of incidence of the ions, and the material of the target, which are the main input variables for the program. From these simulations, it is possible to have an idea of which ions will collide with the surface of the growing film. As a simulation example using this software, $30,000 \mathrm{Ar}^{+}$ions bombarded at normal incidence to a Si target with an energy of $400 \mathrm{eV}$ (a typical energy for Si sputtering), will result in $0.1 \%$ backscattered Ar with an energy distribution from 0 to $5 \mathrm{eV}$. These low values for backscattered atoms show in this case that sputtering induced intermixing will be insignificant. However, it should be accounted for each particular system. In this thesis (Chapter 5), this effect has been considered for Si-on-Ru and Ru-on-Si systems. As mentioned before (Sec. 1.3.2.1), other parameters not accounted on the SRIM software, such as the target-substrate distance, the gas pressure, etc., will also influence the arrival of these energetic particles at the sample surface, provoking damage and roughening (intermixing). A detailed information of damage mechanisms by ions in metals and semiconductors can be found in ref. ${ }^{167}$.

Compound formation. Apart from nucleation and growth of the film, ${ }^{163}$ the arrival of ad-atoms at the substrate can also induce intermixing with the substrate material, if there is chemical affinity to form a compound. As a first approach, it is possible to determine if the reaction process will be thermodynamically more favorable, based on the enthalpies of reaction (or formation). ${ }^{72}$ For instance, sputtered $\mathrm{Ru}$ at room temperature intermixes with $\mathrm{Si}$ (reaction enthalpically favorable), ${ }^{168}$ forming a silicide of several nanometers. ${ }^{145}$ The intermixing by silicide formation can be reduced by passivating the $\mathrm{Si}$ layer by oxygen or nitrogen, forming $\mathrm{SiO}_{2}$ or $\mathrm{SiN}$, as presented in this thesis (Chapter 5). According to thermodynamics, the enthalpies of formation of $\mathrm{SiO}_{2}$ and $\mathrm{SiN}$ are more negative (more favorable) than for RuSi formation. ${ }^{38}$ Thus, the intermixing will be decreased. Thermodynamics are important, although kinetics can play a definitive role in the process, especially at low temperatures. ${ }^{169}$ The kinetic mechanisms of compound formation during growth by magnetron sputtering, are based on the basic thin film kinetics of reaction, combined with surface and in-depth diffusion, which can be found in refs. ${ }^{170,171,172}$. However, each system exhibits its own reaction and inter-diffusion kinetics, and, it should be studied separately.

Since kinetics of reaction are usually faster than diffusion upon deposition, due to the energy of the ad-atoms, diffusion would be the limiting process which determines the resulting intermixing (compound formation). ${ }^{173}$ There are several aspects that can 
influence the diffusion process, for instance, the microstructure (crystallinity) of the substrate or the availability of reactants. An amorphous substrate might allow more interdiffusion with the ad-atoms than a crystalline (or polycrystalline) substrate, for a certain material (ad-atom/substrate) pair, since a disordered structure has more mobility of its atoms than an ordered structure, where the atoms are strongly bounded within the "rigid" lattice. For example, Yulin et al. ${ }^{59}$ observed a larger inter-diffusion in the Mo-Si interface than in the $\mathrm{Si}-\mathrm{Mo}$ interface during growth of $\mathrm{Mo} / \mathrm{Si}$ multilayers, related to the crystallinity of both Si and Mo substrate layers. Si presented an amorphous structure, while Mo was crystalline. The availability of reactants (both ad-atom material and substrate material) might lead to a changing stoichiometry in the formed compound during growth, which results in a change of inter-diffusion. Initially the substrate layer will be the excess reagent, since the ad-atom material is limited. This may result in a substrate-rich compound. However, an ad-atom-rich compound might be formed upon further deposition. Both crystallinity of the substrate and availability of reactants are discussed in this thesis (Chapter 5) for Si-on-Ru and Ru-on-Si systems.

Compound formation is not only limited to the chemical interaction of the ad-atom with the substrate. In reactive sputtering, there is another compound formed upon deposition, as a result of the reaction of the reactive gas (e.g. $\mathrm{O}_{2}$ or $\mathrm{N}_{2}$ ) with the ad-atom. This reaction can occur either in the vapor phase, when both particles encounter, or just when the ad-atom is deposited. A more detailed information of compound formation by reactive magnetron sputtering can be found in ref. ${ }^{174}$. It should be noted that the reactive gas also contributes in the reaction between the ad-atom and the substrate, which usually occurs simultaneously during sputtering, forming a three component inter-diffused layer. The chemical interaction of the $\mathrm{Zr}$ and $\mathrm{O}$ ad-atoms with the a-Si substrate, during reactive sputtering, has been studied in this thesis (Chapter 6).

Surface segregation. Apart from inter-diffusion of ad-atoms with the substrate layer, forming compounds, there is a possibility that some atoms from the substrate layer diffuse up (or segregate) towards the surface of the on-growing layer, motivated by surface effects. Surface atoms exhibit different thermodynamic properties than bulk atoms, due to highly anisotropic chemical environment at the surface compared to the bulk. According to this difference, in multicomponent systems, certain atoms will tend to segregate towards the surface in order to minimize the free energy of the system. ${ }^{165}$ For a single component system, the (specific) surface free energy $G^{S}$ (energy per unit area) is equal to the surface tension $\gamma$. Since the surface tension $\gamma$ is always positive, the specific surface free energy $G^{S}$ is also positive, and it is minimized by keeping the surface tension to a minimum. A high surface tension surface will be covered with a coating of reduced surface tension. ${ }^{165}$ As a first approach, it is possible to consider this simple surface thermodynamics for one component, applied for a multicomponent system, when studying the movement of atoms from the bulk to the surface. Although this is not that 
simple for multicomponent systems, due to other processes occurring simultaneously such as compound formation, it is used in most of the studies (usually in binary systems such binary alloys), to explain this segregation phenomenon. ${ }^{175,176}$ Although thermodynamics are usually at high temperatures, they can help to understand the surface segregation processes at room temperature, even without considering kinetics. However, kinetics should always be accounted. A detailed description of the thermodynamic and kinetic mechanisms of surface segregation can be found in refs. ${ }^{177,178,179}$. Si surface segregation has been observed for Ru grown on $\mathrm{Si}$ (or $\mathrm{SiN}$ ), and for high-O $\mathrm{ZrO}_{2}$ grown on $\mathrm{Si}$ (or $\mathrm{SiN}$ or $\mathrm{SiO}_{2}$ ), as described in Chapters 5 and 6, respectively. In addition, $\mathrm{O}$ surface segregation has also been detected in Chapter 5 for Ru grown on $\mathrm{SiO}_{2}$.

\subsubsection{Current status of growth of metal and metal-oxide thin films}

As mentioned previously in Sec. 1.1.3, both memory and CMOS-like devices normally consist of a Si plug that connects the transistor to the capacitor. ${ }^{6,180}$ Most of photovoltaics also consist of a Si base. ${ }^{47}$ EUVL mirrors also consist of Si alternated by Mo layers, configuring a Mo/Si multilayer stack, which is usually terminated by $\mathrm{Si}^{36,40}$ In all these applications, the oxidation resistant protective material, either a bottom metal electrode, ${ }^{6,85}$ a high-K dielectric layer, ${ }^{22}$ an (oxide) passivation layer, ${ }^{47}$ or a capping layer, ${ }^{40}$ will be normally grown on top of the Si plug, base, or layer, when no diffusion barrier is considered. Typically, this protective material will be either a metallic (e.g., $\mathrm{Pd}, \mathrm{Ru}$, etc.) or a metal-oxide (e.g., $\mathrm{RuO}_{2}, \mathrm{ZrO}_{2}$, etc.) thin film. In particular, this thesis focuses on the study of $\mathrm{Ru}$ and $\mathrm{ZrO}_{2}$, as examples of metallic and metal-oxide thin films, specifically grown on amorphous $\mathrm{Si}(\mathrm{a}-\mathrm{Si})$. This can be relevant for applications where either metals or metal-oxides are deposited on a-Si, such as contact and passivation layers for solar cells, ${ }^{39}$ or as capping layers for EUVL optics. ${ }^{36}$ The current status of growth of both metallic ( $\mathrm{Ru}$ case) and metal-oxide $\left(\mathrm{ZrO}_{2}\right.$ case) thin films will be discussed, as follows.

\subsubsection{Metal thin films: Ru case}

$\mathrm{Ru}$ thin films have been grown on different substrates. ${ }^{181,182,183,184,185}$ Most of the studies are performed by CVD, usually at high temperatures $\left(\sim 200^{\circ} \mathrm{C}\right.$ or higher $)$, and only in few, $\mathrm{Ru}$ has been grown on either natively-oxidized or HF-cleaned $\mathrm{Si}(100){ }^{183,184,185}$ In these last studies, a significant intermixing by $\mathrm{RuSi}_{\mathrm{x}}$ formation is observed during growth. Apart from $\mathrm{RuSi}_{\mathrm{x}}$ formation, other contributions to the intermixing (e.g., surface segregation) have not been considered in such studies. ${ }^{183,184,185}$ However, similar intermixing by $\mathrm{RuSi}_{\mathrm{x}}$ formation might also be present for roomtemperature $\mathrm{Ru}$ grown directly on a-Si. In Chapter 5 of this thesis, the initial growth of sputtered $\mathrm{Ru}$ thin films on a-Si has been studied, including an accurate determination of the surface coverage and the thickness required for closing the Ru layer. In addition, the 
effect of the passivation of the a-Si layer (by nitridation or oxidation) on the Ru growth has been investigated. Thus, similar growth studies have also been performed for $\mathrm{Ru}$ grown on $\mathrm{SiN}$ and $\mathrm{SiO}_{2}$ substrate layers. The in-depth $\mathrm{Ru}$ profiles are also reconstructed from the $\mathrm{Ru}$ surface coverages for $\mathrm{Ru}$ on a-Si, $\mathrm{SiN}$ and $\mathrm{SiO}_{2}$, corresponding to the interdiffusion profiles in all three substrates. In order to distinguish different contributions to the intermixing (as described before on Sec. 1.3.2.3) between $\mathrm{Ru}$ on Si during growth, the reverse system ( $\mathrm{Si}$ on $\mathrm{Ru})$ is also investigated.

\subsubsection{Metal-oxide thin films: $\mathrm{ZrO}_{2}$ case}

$\mathrm{ZrO}_{2}$ thin films have been prepared by several techniques, such as $\mathrm{CVD}^{19,186,187}$ PLD,${ }^{188}$ sol-gel, ${ }^{189,190}$ e-beam evaporation, ${ }^{191}$ or sputtering. ${ }^{192,193,194,195}$ As mentioned in Sec. 1.3.2.2, the film structure and properties are influenced by both deposition process and deposition parameters. In the case of reactive sputtering, Venkarataj et al. ${ }^{157}$ observed a transition from an oxidic sputtering mode (for high oxygen content) to a metallic sputtering mode (for low oxygen content), which resulted into different optical and structural properties of the grown $\mathrm{ZrO}_{2}$ films. In that study and in the other reported deposition studies, $\mathrm{ZrO}_{2}$ thin films were grown on either natively-oxidized or HF-cleaned $\mathrm{Si}(100)$ substrates. A stable silicate interlayer at the $\mathrm{ZrO}_{2} / \mathrm{Si}$ interface was formed during growth, even at enhanced temperature $\left(200^{\circ} \mathrm{C}\right.$ or higher $)$ deposition conditions. ${ }^{19}$ This silicate interlayer might also form in the case of room-temperature $\mathrm{ZrO}_{2}$ deposition on a$\mathrm{Si}$. In Chapter 6 of this thesis, the initial stages of growth of reactive sputtered $\mathrm{ZrO}_{2}$ thin films directly on a-Si, have been studied for two gas deposition condition, with high and low oxygen content, representing the two mentioned sputtering modes. As in the case of $\mathrm{Ru}, \mathrm{ZrO}_{2}$ growth has also been investigated on top of $\mathrm{SiN}$ and $\mathrm{SiO}_{2}$ substrate layers for high oxygen and low oxygen conditions, in order to study the influence of the $\mathrm{Si}$ passivation on the $\mathrm{ZrO}_{2}$ growth. In addition, the $\mathrm{ZrO}_{2}$ stoichiometry, density and its initial interaction (compound formation, surface segregation) with the underneath a-Si layer, have been determined for both conditions.

\subsection{Oxidation of thin films}

\subsubsection{Oxidation methods}

There are several methods for (post-)oxidation of thin films through gas vapor-solid phase interaction such as atomic oxygen beam source oxidation, ${ }^{196}$ oxygen ion bombardment, ${ }^{197}$ plasma-assisted oxidation, ${ }^{198,199}$ EUV-assisted oxidation (or similar ebeam-assisted oxidation), ${ }^{200,201}$ and thermal oxidation. ${ }^{113}$ In all of them, there are two elements required: an oxygen source (molecular oxygen, atomic oxygen, oxygen ion, ozone, water vapor, hydrogen peroxide vapor) and an energy source (electron beam, laser beam, EUV or UV light, plasma discharge, thermal cracking, thermal annealing). From 
these methods, the relevant ones for this thesis are: EUV-assisted oxidation and thermal oxidation, as described as follows.

EUV-assisted oxidation (or similar e-beam-assisted oxidation). EUV-assisted oxidation is driven by EUV photon assisted direct or secondary electron induced fragmentation of surface adsorbed water, forming atomic oxygen, which causes thin film oxidation. ${ }^{200}$ Similar phenomenon is occurring in the mirrors' surface in the EUVL systems, as described in Sec. 1.1.3. However, in the case of EUVL systems, there is not only water vapor, but also hydrocarbons, hydrogen, and other contaminants, which make the process more complex. Nevertheless, EUV-assisted oxidation in presence of water vapor is not a very efficient way of oxidizing a thin film, since an EUV source is required for having a similar effect as using directly an atomic oxygen beam source. However, the process is more complex, since EUV light induces the formation of surface water plasma that, not only consists of atomic $\mathrm{O}$, but also other reactive species $\left(\mathrm{H}^{+}, \mathrm{OH}^{-}, \mathrm{O}^{-}\right.$, etc.), mainly due to the effect of secondary electrons emitted from the film with energies less than $20 \mathrm{eV}$, and not because of the direct photon excitation. ${ }^{202}$ Due to the limited access to EUV sources or synchrotron beamlines, similar oxidation is pursued by means of electron beams in presence of water vapor, ${ }^{201,203}$ as observed in several studies. ${ }^{49,128,132,204}$ However, the process occurring might be somehow different from using EUV radiation, since photons are not present.

Thermal oxidation. This method uses an oxidizing gas that diffuses into the film and reacts with it at enhanced temperatures, causing its oxidation. Depending on the oxidizing gas, there are different types of thermal oxidation. The typical oxidizing gases used are $\mathrm{O}_{2}$ or $\mathrm{H}_{2} \mathrm{O}$ vapor, which lead to the so-called dry or wet oxidation, respectively. ${ }^{113}$ A simple variant of "dry" oxidation is by means of atmospheric air which contains on average $<1 \%$ water vapor. It should be noted that air mainly consists of $\mathrm{N}_{2}$ (78.09\%), but also other gases such as $\operatorname{Ar}(0.93 \%)$ or $\mathrm{CO}_{2}(0.04 \%)$, and only $20.95 \%$ is $\mathrm{O}_{2} \cdot{ }^{38}$ However, since $\mathrm{N}_{2}$ and Ar normally are inert gases, the main oxidizing agent is $\mathrm{O}_{2}$, which can lead to similar oxidation as by using only $\mathrm{O}_{2}$, although other side subprocesses might occur due to the air mixture. Thermal oxidation can be performed in ovens or furnaces with a constant flow of either $\mathrm{O}_{2}$, air or $\mathrm{H}_{2} \mathrm{O}$ vapor, and normally a constant temperature. Traditionally, ovens operate at temperatures below $540^{\circ} \mathrm{C}$ $\left(1000^{\circ} \mathrm{F}\right)$ and furnaces can run up to $1100^{\circ} \mathrm{C}\left(2000^{\circ} \mathrm{F}\right)$ or higher. ${ }^{113}$ Thermal oxidation can also be performed by heating the sample substrate in a vacuum chamber with a more controlled $\mathrm{O}_{2}$, air, or $\mathrm{H}_{2} \mathrm{O}$ vapor atmosphere. ${ }^{205}$ Dry oxidation produces higher-quality films with higher density than wet oxidation and, it is normally used for producing thin oxide films. Both dry and wet oxidation are normally used in microfabrication to produce a thin oxide layer (normally $\mathrm{SiO}_{2}$ ) on the surface of the wafer. Wet oxidation is preferred to dry oxidation for growing thick oxides, due to its higher growth rate. However, it produces more dangling bonds at the silicon interface (the so-called "dirty" interface), 
which yields to a lower quality oxide with lower density. Thus, thick oxides in microelectronics are normally produced by a long wet oxidation cycle spaced by short "dry" ones (a dry-wet-dry cycle). ${ }^{113}$

\subsubsection{Choice of oxidation technique}

From all oxidation techniques, the most related technique to microelectronics (DRAMs, CMOS devices) would be thermal ("dry" or "wet") oxidation. For photovoltaics, also this oxidation method would be relevant together with a UV source, similar to the sunlight exposure. In the case of EUVL optics, the use of EUV-assisted oxidation (or similar e-beam-assisted oxidation) in presence of water vapor would be the most significant oxidation technique. However, as described before in Sec. 1.4.1, the oxidation process is not easily controlled, and possible to understand, due to the amount of processes occurring at the surface due to surface-induced plasma. A similar problematic is occurring for the plasma-assisted oxidation, where, for instance, $\mathrm{Ru}$ etching is an added issue. ${ }^{206,207}$ Ion-bombardment is also not well controlled, due to extra mechanisms occurring in parallel with the oxidation process, such as ion-implantation. ${ }^{197}$ Atomic oxygen, without an additional thermal load, is not powerful enough to provoke in-depth oxidation (of few nanometers) in certain materials, disabling its use for oxidation studies. For instance, oxidation of Ru thin film by atomic oxygen is restricted to the near-surface ( $<1 \mathrm{~nm}$ oxide formed), as observed in ref. ${ }^{133}$. To enhance the oxidation process, making it simple to control, instead of adding a third parameter (for instance, temperature) to the atomic oxygen exposure, it is by using thermal oxidation, relevant for all microelectronic, photovoltaic, and EUVL applications. This simple method (in the case of "dry" oxidation), with only two parameters (oxygen content "from air" and temperature), is the one used in this thesis for studying surface and sub-surface oxidation (oxygen diffusion) of Ru thin films (Chapter 3 and Chapter 4), and oxygen diffusion in $\mathrm{ZrO}_{2}$ thin films (Chapter 6).

The processes occurring by thermal oxidation might differ from the processes taking place during, cyclical thermal loading with UV sunlight in atmospheric exposure, or, during EUV-induced oxidation. However, by understanding the processes occurring during thermal oxidation, we can understand better the oxidation processes taking place during fabrication of DRAMs, CMOS devices and solar cells, and also during their operation (including the EUVL optics operation), since in all cases, the main elements are present: oxygen source (molecular oxygen, atomic oxygen, oxygen ion, ozone, water vapor, hydrogen peroxide vapor, or oxygen content "from air") and energy source (electron beam, laser beam, EUV/UV light, plasma discharge, or temperature). In addition, in the case of EUVL mirrors, after long exposure, and especially the ones close to the EUV source, might reach high temperatures, leading to enhanced oxidation. ${ }^{36}$ In this case, the oxidation phenomena could be partially understood from the thermal 
oxidation studies. In the next section, thermal oxidation (the "dry" one) will be explained more in-depth, including the main processes occurring during thermal oxidation and their associated kinetic mechanisms. Note that the basic thermodynamics of oxidation are already extensively described in Sec. 1.2.2.

\subsubsection{Thermal oxidation ("dry" case)}

There are different processes occurring during thermal oxidation. In order to englobe these processes for all material types, it is interesting to study the general case of pure materials, such as metals. Thus, the main processes occurring during thermal oxidation of metals (considering only $\mathrm{O}_{2}$ ) are explained in the following section, including their kinetic mechanisms.

\subsubsection{Main processes occurring during oxidation $\left(\mathrm{O}_{2}\right)$ of metals}

From the different processes occurring during metal oxidation, the main processes are illustrated in the Fig. 1.3, and described as follows:

1. Oxygen impingement. This process consists of the arrival of $\mathrm{O}_{2}$ from the gas phase at the metal surface. The number of gas molecules $N$ colliding (in one direction) a surface $S$ per unit area per unit time $t$ (or "impingement rate" $r_{\text {coll-surf }}$ ) can be derived from the kinetic theory, ${ }^{208}$ when assuming an ideal gas as:

$$
r_{\text {coll-surf }}=\frac{1}{S} \frac{d N}{d t}=\frac{p}{\sqrt{2 \pi m k_{B} T}},
$$

where $p$ is the gas pressure, $m$ is the mass of a gas molecule, $k_{B}$ is the Boltzmann constant, and $T$ is the absolute temperature (in $\mathrm{K}$ ). In the case of $\mathrm{O}_{2}, \sim 2.7 \times 10^{27}$ molecules $\cdot \mathrm{m}^{-2} \cdot \mathrm{s}^{-1}$ will be hitting a metal surface at ambient conditions $\left(1 \mathrm{~atm}\right.$ and $\left.25^{\circ} \mathrm{C}\right)$, when applying Eq. (1.4). When considering $\mathrm{Ru}$, as a relevant material for this thesis, this value would mean that on average each $\mathrm{Ru}$ surface atom will be hit $\sim 1.7 \times 10^{8}$ times per second by $\mathrm{O}_{2}$ under ambient conditions, when assuming an average surface density of $\sim 1.58 \times 10^{19}$ atoms $\cdot \mathrm{m}^{-2} \cdot{ }^{209}$ However, this does not mean that each $\mathrm{O}_{2}$ molecule sticks to the $\mathrm{Ru}$ surface, since it is usually an activated process, as later described in this section. 


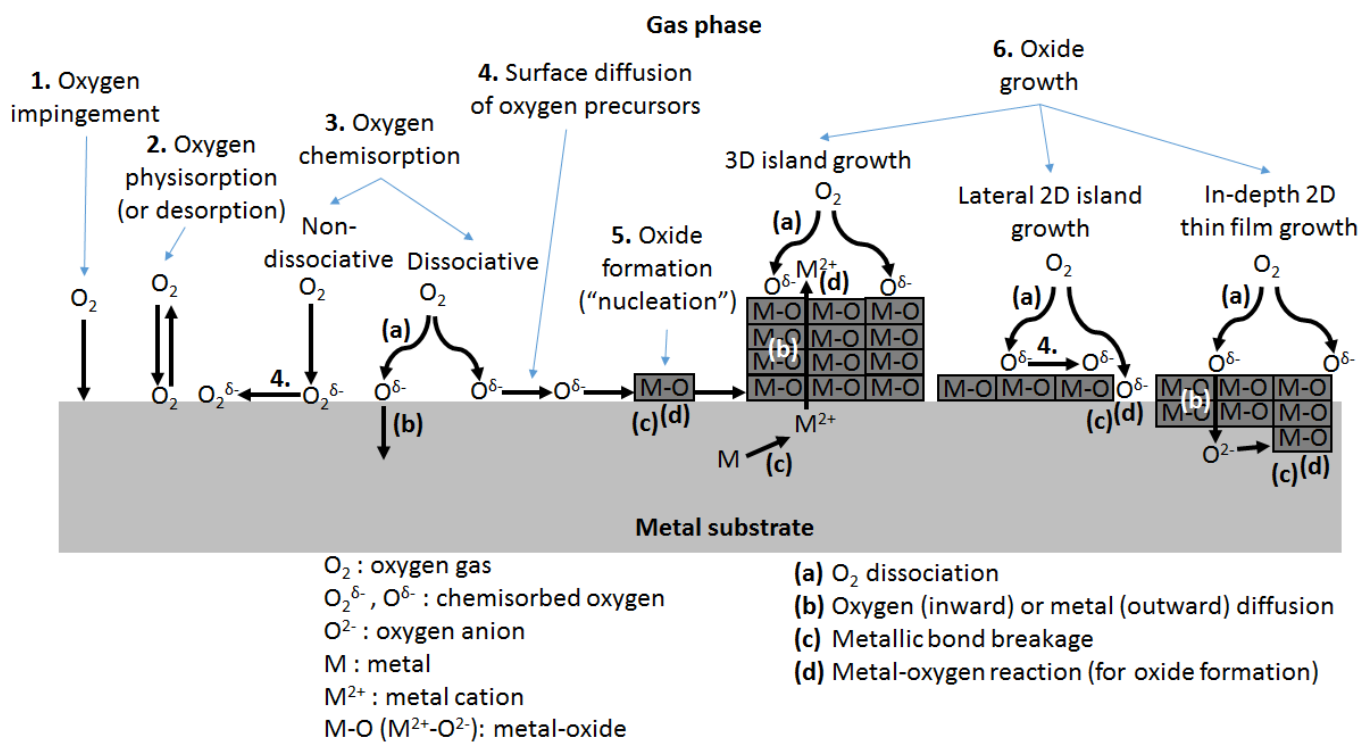

Fig. 1.3. Scheme of main processes occurring during metal oxidation.

2. Oxygen physisorption (or desorption). The gas molecules arriving at the surface will experience a spontaneous weak interaction with the surface adsorption sites by van der Waals forces, which will allow to adsorb physically (physisorption, or also called non-reactive adsorption). But due to the weakness of these forces, there will be a dynamic equilibrium between adsorption and desorption. This dynamism will allow formation of physisorbed gas multilayers. ${ }^{210}$ However, since oxygen strongly reacts with many metals, it will normally adsorb chemically (chemisorption) on the metal surface at ambient conditions, since this later interaction is stronger than physisorption. For instance, $\mathrm{Ru}$ forms about $\sim 1 \mathrm{ML}^{211}$ of chemisorbed oxide at ambient conditions. ${ }^{76}$

3. Oxygen chemisorption. As mentioned, the $\mathrm{O}_{2}$ molecules arriving at the surface will strongly interact with the metal surface, forming chemical bonds. As a difference with physisorption, this process will usually not occur spontaneously and an extra energy source (for instance, thermal or by having a catalytic surface such as Ru) ${ }^{14}$ will be required. In addition, the process is normally irreversible and often limited to $1 \mathrm{ML},{ }^{211}$ contrarily to physisorption where desorption also occurs and several monolayers can be formed. ${ }^{210}$ There are two types of chemisorption, depending on if the gas adsorbs on the surface directly in its molecular form, or undergoes a dissociation process before is adsorbed, leading to the so-called non-dissociative or dissociative chemisorption, respectively. ${ }^{212}$ Since usually diatomic gases such as $\mathrm{H}_{2}, \mathrm{~N}_{2}$ or $\mathrm{O}_{2}$ undergo dissociation upon adsorption on several metals (for instance, $\mathrm{Ru}$ which is the relevant metal in this thesis), ${ }^{213,214,215}$ only dissociative chemisorption will be discussed, as follows. 
Dissociative chemisorption. This type of chemisorption can proceed via a direct or an indirect process. For the direct process, the dissociation occurs just on the first initial collision of the gas molecule with the surface. Although it is the most common dissociative chemisorption process of gases such as $\mathrm{H}_{2}$ and $\mathrm{N}_{2}$ on metal surfaces, ${ }^{212} \mathrm{O}_{2}$ chemisorption on $\mathrm{Ru}$ undergoes via a precursor-mediated mechanism, ${ }^{216}$ the indirect process. For the indirect process, the dissociation is preceded by the formation of a surface precursor that either diffuses freely across the surface to find a favorable dissociation site, or its mobility is limited to the place where the precursor is initially formed. ${ }^{215}$ The last path is the one occurring for most systems, since the precursor mobility is normally limited, and depending on the surface coverage. ${ }^{212}$ The precursormediated mechanism with limited precursor mobility usually includes two steps. There is an initial adsorption step of the gas molecule $\mathrm{A}_{2}$ on a surface site (*), forming a rather immobile molecular precursor, followed by a dissociation step with a neighboring site:

$$
A_{2}+2 * \rightarrow A_{2}^{*}+* \rightarrow 2 A^{*}
$$

This mechanism can follow a $1^{\text {st }}$ order Langmuir adsorption kinetics if the limiting step is the adsorption step. If this is the case, the next expression can describe this kinetics, written in terms of surface coverage $\theta_{A^{*}}$, and expressed as surface atomic fraction by:

$$
\frac{d \theta_{A *}}{d t}=\frac{2}{N_{0}} \frac{p}{\sqrt{2 \pi m k_{B} T}} S_{0}\left(1-\theta_{A *}\right),{ }^{212}
$$

where $\theta_{A}{ }^{*}=N_{A} / M, N_{A}$ is the number of surface sites (*) occupied by atoms $A, N_{0}$ is the total number of adsorption sites $M$ per area $S, p / \sqrt{2 \pi m k_{B} T}$ is the "impingement rate" $r_{\text {coll-surf }}$ from Eq. (1.4), and $S_{0}$ is the initial sticking coefficient (for $\theta_{A^{*}}=0$ ). $S_{0}$ varies from 1 (when the precursor is totally free to move along the surface) down to $10^{-3}$ or even lower (when the precursor is completely immobilized). Although, the indirect dissociative chemisorption with a limited mobility precursor can be a more complex process, depending on the precursor nature (extrinsic or intrinsic) and the paths for its formation. ${ }^{217}$ It should be noted that kinetic models for dissociative chemisorption are based on simple Langmuir kinetics which might be valid only in some cases. Other kinetic models can be found in refs. ${ }^{212,218}$

In the case of adsorption of $\mathrm{O}_{2}$ on $\mathrm{Ru}$, which undergoes via a limited precursormediated dissociative chemisorption, there are several studies on kinetics of $\mathrm{O}_{2}$ adsorption on monocrystalline $\mathrm{Ru} .{ }^{215,216,219,220,221}$ However, to the best of our knowledge, there is no reported data for oxygen adsorption kinetics on polycrystalline $\mathrm{Ru}$ at room temperature. Polycrystalline $\mathrm{Ru}$ is relevant due to that the structure of $\mathrm{Ru}$ thin films used as oxidation resistant protective layers for DRAMs, CMOS devices, solar cells, or EUVL optics is either polycrystalline or amorphous rather than monocrystalline. Thus, the adsorption process of oxygen on sputter cleaned polycrystalline Ru was investigated by 
in situ low-energy ion scattering, and it was found to be similar to oxygen adsorption on monocrystalline $\mathrm{Ru}^{222}$

4. Surface diffusion of oxygen precursors. After $\sim 1 \mathrm{ML}$ chemisorbed oxygen is achieved on the metal surface, there can be some processes of surface diffusion (transport) of oxygen precursors at enhanced temperatures under $\mathrm{O}_{2}$ atmosphere. After a dynamic process, these oxygen precursors might collapse with the metal surface atoms, forming strong bounds with the metal matrix (oxide formation), usually motivated by the presence of heterogeneities on the surface such as step bunching regions. ${ }^{223}$ This process of oxide formation, in most metals, corresponds to stoichiometric oxide formation. ${ }^{54}$ In some cases, the oxygen precursors are undefined labile metal-oxygen structures, with more or less mobility, similar to free-standing amorphous-like non-stoichiometric oxide structures. They are originated by the detachment of some metal atoms from the metallic matrix that diffuse and react with atomic oxygen species, formed by oxygen dissociation from the gas phase. ${ }^{224}$ For instance, an O-Ru-O trilayer has been proposed by DFT calculations as the precursor for surface oxidation of $\mathrm{Ru}(001)$, without additional experimental evidence. ${ }^{225}$ However, recent experimental data on thermal oxidation of $\mathrm{Ru}(001)$ by $\mathrm{NO}_{2}$ reported that this trilayer precursor is an unreconstructed $\mathrm{RuO}_{2}(100)$ surface oxide with possible structural disorder. ${ }^{226}$ This precursor structure resembles our initial amorphous chemisorbed oxide layer formed on polycrystalline $\mathrm{Ru}$ surface upon room temperature atmospheric exposure (departing structure for oxidation studies in Chapter 3). Although it is already an oxide, originated most probably due to an electrochemical path, ${ }^{76,222}$ it is still limited to $\sim 1 \mathrm{ML}^{211}$ oxide, with a rather disordered and non-stoichiometric structure. It does also not evolve further at room temperature, most probably due to kinetic barriers that impede further oxidation, stoichiometric oxide formation, or even crystallization.

5. Oxide formation. As just mentioned, the existence of oxygen precursors that diffuse along the surface with a rather high-mobility, ${ }^{224}$ can lead to the formation of surface oxide. Normally, this process can be described by a simple "nucleation and growth mechanism", 227,228 involving accumulation of such precursor species at certain surface defects, where an oxide can evolve. ${ }^{223}$ This mechanism begins with a dynamic process between clustering and dissolution of precursors. These dynamic clusters are normally called nuclei. However, only when these nuclei reach a certain critical size, they become stable, without possible re-dissolution ("nucleation"), and can continue to grow in size ("growth"). These processes occurring at the surface that can lead to normally stoichiometric oxide formation are activated, requiring enhanced temperatures and enhanced $\mathrm{O}_{2}$ pressures (and doses), depending on the metal. In the case of $\mathrm{Ru}$, certain temperatures are needed to open two neighboring adsorption sites on its surface for $\mathrm{O}_{2}$ dissociation, ${ }^{224}$ which seems limited at coverages $>0.5 \mathrm{ML}$ chemisorbed oxygen, ${ }^{216}$ and also to promote the formation and subsequent diffusion of metal-oxygen precursors on 
the surface, as discussed in this thesis (Chapter 3). At the same time, certain pressures are needed to allow the stabilization of a critical $\mathrm{RuO}_{2}$ nucleus, that can initiate its growth at a specific temperature. ${ }^{14}$ For instance, $\mathrm{Ru}(001)$ oxidation (stoichiometric $\mathrm{RuO}_{2}(110)$ formation) requires a threshold temperature of $\sim 230^{\circ} \mathrm{C}^{229}$ and a threshold pressure of $\sim 1$ x $10^{-5} \mathrm{mbar}_{2}\left(>4 \times 10^{4} \mathrm{~L}\right) .{ }^{230}$ Below this threshold temperature, in the region between $\sim 130$ to $\sim 230^{\circ} \mathrm{C}$, only an amorphous oxygen-rich phase with subsurface oxygen (a socalled transient surface oxygen (TSO)) has been suggested by Blume et al. ${ }^{205,231}$ to develop on $\mathrm{Ru}(001)$ surface. In these studies, ${ }^{205,231}$ this TSO phase has been associated to the mentioned O-Ru-O trilayer, ${ }^{225}$ (for $\mathrm{O}_{2}$ exposure), or in other studies, to a comparable distorted $\mathrm{RuO}_{2}$ precursor structure (for $\mathrm{NO}_{2}$ exposure). ${ }^{226}$ It also resembles the amorphous, oxygen-rich, low density $\mathrm{RuO}_{\mathrm{x}}(2<\mathrm{x} \leq 3)$ layer formed on polycrystalline $\mathrm{Ru}$, below a threshold temperature for surface $\mathrm{RuO}_{2}$ nucleation $\left(\sim 200^{\circ} \mathrm{C}\right)$, as experimentally observed in our oxidation studies presented in this thesis (Chapter 3 ).

6. Oxide growth. After the first metal-oxide nucleus is formed ("nucleation") on the chemisorbed oxygen layer, it can continue to grow further, as long as there is enough energy (thermal), and both oxygen (usually atomic $\mathrm{O}$ ) and metal supplies. In the case of $\mathrm{Ru}(001)$ oxidation, although there is a very low $(<0.04)$ initial sticking coefficient for $>0.5 \mathrm{ML}$ chemisorbed oxygen, ${ }^{216}$ as soon as the first oxide nucleus (or initial $\mathrm{RuO}_{2}(110)$ cluster) appears on its surface, it will continue growing autocatalytically, ${ }^{232}$ since the initial sticking coefficient becomes $\sim 0.7$ on $\mathrm{RuO}_{2}(110)$, even at room temperature. ${ }^{233}$ Thus, the formation of a surface $\mathrm{RuO}_{2}$ nuclei, catalyze the $\mathrm{O}_{2}$ dissociation, selfaccelerating the oxide growth process. ${ }^{234}$ This results in a self-limited growth of $\sim 1.6$ nm-thick $\mathrm{RuO}_{2}(110)$ layer on $\mathrm{Ru}(001)$ surface over a large temperature, and $\mathrm{O}_{2}$ pressure range from $\sim 280$ to $\sim 380^{\circ} \mathrm{C}$, and $10^{-4}$ to 10 mbar, respectively. ${ }^{230}$ However, since this autocatalytic oxidation process for $\mathrm{Ru}$ and, other metals such as $\mathrm{Pb},{ }^{235}$ in general, occurs at enhanced temperatures, where the kinetic barriers for oxide formation ("nucleation") are overcome, thermodynamics become more relevant. Thus, oxide growth will be driven by the reduction of the total Gibbs free energy of the system, ${ }^{236}$ which will lead to a certain growth type (similar to the so-called growth mode in thin film growth), ${ }^{164}$ limited always to its growth kinetics. The three main growth types include: 3D island growth, lateral 2D island growth, and in-depth 2D thin film growth, and are discussed as follows.

3D island growth. As depicted in the scheme presented in Fig. 1.3, the growth of $3 \mathrm{D}$ islands at the metal surface involves breakage of a metallic bond (c), diffusion of the metal (outward) through the oxide (b), $\mathrm{O}_{2}$ dissociation (a), and metal-oxygen reaction at the gas-oxide interface (d). In general, this growth is anisotropic, since diffusion is usually different in the out- and in- plane direction. For instance, an oxidation study revealed the formation of surface $3 \mathrm{D}$ islands $\left(\mathrm{RuO}_{2}\right.$ flakes $)$ on a fully covered $\mathrm{RuO}_{2}(110)$ layer on $\mathrm{Ru}(001)$, with an average lateral island size and island height of $20 \mathrm{~nm}$ and 10 $\mathrm{nm}$, respectively, when exposed to $5 \times 10^{-5} \mathrm{mbar}_{2}$ at $\sim 410^{\circ} \mathrm{C}$ during $960 \mathrm{~s}^{223}$ It also 
was observed in refs. ${ }^{230,237,238}$, showing the clear anisotropic character of 3D island growth. This growth type is also observed in this thesis (Chapter 3) for thermally oxidized polycrystalline $\mathrm{Ru}$.

Lateral 2D island growth. As shown in Fig. 1.3, the lateral growth of 2D islands on the metal surface requires $\mathrm{O}_{2}$ dissociation (a), usually followed by surface diffusion of oxygen precursors (4.), metallic bond breakage (c), and oxide formation reaction at the gas-metal interface (d). Considering an epitaxial oxide growth, this lateral 2D island growth usually precedes the formation of a flat oxide, after coalescence of various 2D islands, occupying the full metal surface. For instance, after $>4000 \mathrm{~s}_{2}$ exposure on $\mathrm{Ru}(001)$ surface at $\sim 3 \times 10^{-5} \mathrm{mbar}$ and $\sim 410^{\circ} \mathrm{C}$, the surface is completely covered by coalescent $2 \mathrm{D} \mathrm{RuO}_{2}$ islands, forming a thin flat $\mathrm{RuO}_{2}$ layer. ${ }^{223}$ Although this growth might also be present in thermal oxidation of polycrystalline $\mathrm{Ru}$ (Chapter 3), we could only monitor after the formation of the thin flat oxide, since at atmospheric conditions $\sim 1 \mathrm{ML}$ chemisorbed oxide is immediately formed. ${ }^{76}$

In-depth 2D thin film growth. Usually after the surface is covered by a flat oxide layer, even there are 3D islands on its top, this oxide layer can continue growing in-depth (sub-surface oxide growth), normally by $2 \mathrm{D}$ thin film growth. This process is depicted in Fig. 1.3, where there is $\mathrm{O}_{2}$ dissociation on the oxide surface (a), followed by oxygen diffusion (inward) through the oxide layer (b), metallic bond breakage (c), and metaloxygen reaction at the oxide-metal interface (d). For instance, in-depth $2 \mathrm{D}$ thin film growth of $\mathrm{RuO}_{2}(110)$ flat layer on $\mathrm{Ru}(001)$, with a thickness larger than the mentioned $1.6 \mathrm{~nm}$ self-limiting oxide value, has been observed for temperatures $>380^{\circ} \mathrm{C}$ at $\mathrm{O}_{2}$

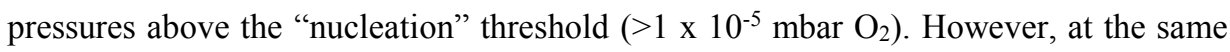
time there has been observed a significant faceting of the $\mathrm{RuO}_{2}$ film, without apparent lateral $\mathrm{RuO}_{2}$ domain size change. ${ }^{230}$ It should be noted that in-depth $2 \mathrm{D}$ thin film growth might also undergo by metal (outward) diffusion through the oxide and reaction at the gas-oxide interface, similar as in the case of 3D island growth (Fig. 1.3), but with a homogeneous growth front in the out- and in-plane direction, characteristic from a $2 \mathrm{D}$ growth. In this case, the oxide would seem to grow towards the surface from the bottom, but the metal-oxide interface will still continue shifting in-depth. The last possibility is having both (oxygen and metal) diffusing (inward and outward), still producing a 2D thin film oxide. This last is the case observed in our study (Chapter 3 ) for in-depth 2D thin film $\mathrm{RuO}_{2}$ growth, where there is both oxygen (inward) and ruthenium (outward) diffusion through the evolving $2 \mathrm{D}$ thin film oxide, occurring simultaneously with the previously mentioned 3D oxide growth type.

From the different processes occurring during the three discussed oxide growth types for metal oxidation (see scheme in Fig. 1.3), diffusion through the oxide either of the oxygen (inward or surface lateral) or the metal (outward) (b) is usually the kinetically 
limiting process for oxide growth at enhanced temperatures higher (one or various hundred ${ }^{\circ} \mathrm{C}$ ) than the threshold for oxide formation ("nucleation") ${ }^{103} \mathrm{O}_{2}$ dissociation (a), metallic bond breakage (c), and metal-oxygen reaction (d) are normally several orders of magnitude faster at these temperatures, especially on the already formed oxide. ${ }^{235,239}$ For instance, the self-accelerated autocatalytic $\mathrm{RuO}_{2}(110)$ growth after the first oxide nucleus is formed on $\mathrm{Ru}(001)$ surface. ${ }^{232}$ Since diffusion through the oxide, as a mass transport phenomenon (of oxygen or metal), is usually slower than the other mechanisms occurring during high temperature metal oxidation (Fig. 1.3), it is the process that controls and limits the oxide growth rate, and hence the overall oxidation process. However, within the diffusion process, the fastest diffusing species (oxygen or metal) will determine the oxide growth rate. In the case of in-depth $2 \mathrm{D}$ thin film oxide growth on polycrystalline $\mathrm{Ru}$, where both species may diffuse, the oxide growth rate seems determined by ruthenium upwards diffusion (usually via $\mathrm{Ru}^{4+}$ ), being the fastest species diffusing, as suggested from the experimental data shown in this thesis (Chapter 3 ). In the case of 3D island oxide growth, this ruthenium species clearly determines the growth, since the oxidation reaction might take place at the gas-oxide interface, without apparent in-depth oxygen diffusion (usually via $\mathrm{O}^{2-}$ ) (Chapter 3 ). In this thesis we are interested to understand the in-depth oxygen transport through the oxidation resistant protective layer $\left(\mathrm{RuO}_{2}\right.$ (Chapter 4) or $\mathrm{ZrO}_{2}$ (Chapter 6)) to be able to prevent the oxidation of the underneath layers in microelectronics (DRAMs, CMOS devices), solar cells, and EUVL optics, which would lead to a decrease of the effective dielectric constant, a reduction of the UV transmission and the photo-generated power via recombination and electrical losses, and a loss of the EUV reflectance, respectively. However, since in this thesis we could not distinguish between either oxygen (anions) or metal (cations) diffusion through the oxide, the overall diffusion is expressed by an effective diffusion constant $D_{\text {eff }}$ (referred as $D$ in Chapter 4), which describes the in-depth growth of the oxide film, and hence the in-depth "oxygen" diffusion process. In the next section (Sec. 1.4.3), the diffusion-limited growth of oxide thin films will be discussed, including the main factors influencing $D_{\text {eff }}$ (Sec. 1.4.3.1). In addition, the current status of Ru thin films oxidation, attending to the diffusion-limited growth, and the current status of oxygen diffusion through $\mathrm{RuO}_{2}$ and $\mathrm{ZrO}_{2}$ thin films will be also presented in Secs. 1.4.3.2 and 1.4.3.3, respectively.

\subsubsection{Diffusion-limited growth of oxide thin films}

As described in the previous section (Sec. 1.4.2.1), at high temperatures the growth of the metal-oxide thin films is limited by the diffusion through the film of metal (cations), or oxygen (anions), or both. For most of oxide thin films, which do not contain macroscopic defects (such as porosities or cracks), this process could be best described by an empirical relationship such as: 


$$
z^{2}=D_{e f f} \cdot t^{240}
$$

where $z$ is the oxide thickness, $t$ is the annealing time, and $D_{\text {eff }}$ is an effective diffusion constant (or rate), related to the real diffusion constant $D_{\text {real }}$. Eq. (1.7) is well-known in the theory of oxidation of metals as the parabolic growth law. ${ }^{240}$ It was firstly introduced by Pilling and Bedworth in $1923,{ }^{241}$ where $D_{\text {eff }}$ was defined as:

$$
D_{\text {eff }}=2 R \cdot \Delta C \cdot D_{\text {real }},
$$

where, $R$ is the ratio between the volume of the oxide and the volume of the diffusing species, and $\Delta C$ is the difference of concentration of diffusing species between the metaloxide and the oxide-gas interfaces (typical diffusion driving force). This expression (combined Eqs. (1.7) and (1.8)) could be derived from the application of the Fick's first law of diffusion, when considering the following assumptions:

- Growth occurs by uncharged particles.

- $D_{\text {real }}$ is independent of the concentration $C$.

- Concentration $C$ at both interfaces is independent of the thickness $z$.

- Growth is a steady state phenomenon.

Although in this explanation from Pilling and Bedworth (combined Eqs. (1.7) and (1.8)) for the empirical relationship (Eq. (1.7)) charged particles were not taken into account (usually the species diffusing in ionic metallic oxides), it served as a first reasoning to the observation, that later was culminated by the integrated theory from Fromhold et at. ${ }^{242}$ (considering also the contribution of the charges). It should be noted that Eq. (1.7) assumes $D_{\text {eff }}$ to be constant and independent of the oxide thickness $z$. However, in our oxygen diffusion study through $\mathrm{RuO}_{2}$ presented in this thesis (Chapter 4), we have experimentally demonstrated that the effective diffusion rate $D_{\text {eff }}$ for this process is not constant, and depends on $z$. This can be associated to a gradual change in the polycrystalline structure of the growing $\mathrm{RuO}_{2}$, as described in Chapter 4. Thus, for each oxide thickness $z$, there is an associated effective diffusion rate $D_{\text {eff. Note that }} D_{\text {eff }}$ is referred in Chapter 4 as $D$. Apart from the oxide thickness $z$, there are other factors, such as the mentioned oxide polycrystalline structure, that can influence the effective diffusion rate $D_{\text {eff }}$, and hence the real diffusion constant $D_{\text {real }}$, since $D_{\text {real }} \alpha D_{\text {eff. }}$. The main factors influencing $D_{e f f}$ are discussed in the next section.

\subsubsection{Main factors influencing $D_{\text {eff }}$}

The three main factors which are relevant for this thesis are: the size of the diffusing species (solute), the oxide (host) microstructure, and the temperature, which are described as follows. 
Size of the diffusing species. Larger species will diffuse slower than smaller species, since they are less mobile and need larger paths for diffusion within the host (assuming similar diffusion mechanism). However, apart from the size, the charge is also important, which gives an extra driving force for diffusion. For instance, diffusion of carbon ${ }^{243}$ is $\sim 10^{5}$ times slower than of oxygen $\left(\mathrm{O}^{2-}\right)^{244}$ in sintered $\mathrm{ZrO}_{2}$ powder at $900^{\circ} \mathrm{C}$. Although $\mathrm{C}$ is much smaller than $\mathrm{O}^{2-}$, the charge was absent in the implanted $\mathrm{C}$ in that study, ${ }^{243}$ leading to slower diffusion, and showing the lower diffusivity of insoluble $\mathrm{C}$ in $\mathrm{ZrO}_{2}$, compared to $\mathrm{O}^{2-}$. It should be noted that cations are usually smaller than anions (from the ionic radius), leading to faster diffusion rates depending on their charge. For instance, $\mathrm{Ru}^{4+}$ and $\mathrm{O}^{2-}$ ionic radius are 0.6 and $1.4 \AA$, respectively. ${ }^{245}$ This means that according to the size and the charge, $\mathrm{Ru}^{4+}$ will diffuse faster than $\mathrm{O}^{2-}$, as clearly observed in the anisotropic growth of surface 3D $\mathrm{RuO}_{2}$ nano-columns (Chapter 3). This is not that obvious in the case of in-depth $2 \mathrm{D}$ thin film $\mathrm{RuO}_{2}$ growth, due to the experimental difficulties to distinguish from upward or inward film oxide growth. However, in both cases, diffusion will be strongly determined by the oxide microstructure that is presented as follows.

Oxide microstructure. In oxides, as in other solids, diffusion undergoes through defects in the (host) structure that provide paths for the diffusing species to penetrate through. The amount and kind of defects depends on the microstructure, which will lead to a certain diffusion mechanism, and hence certain diffusion kinetics. Thus, defects and diffusion mechanisms (and kinetics) for the three main types of oxide microstructures (monocrystalline, polycrystalline and amorphous) are discussed as follows. It should be noted that although the oxide microstructure of $\mathrm{RuO}_{2}$ and $\mathrm{ZrO}_{2}$ protective layers, relevant in this thesis, is either polycrystalline or amorphous, the presence of various monocrystals integrated in a rather polycrystalline or amorphous-like matrix, makes monocrystalline oxides, and its associated diffusion mechanisms, also relevant for the thesis.

1. Monocrystalline oxides. Diffusion in single crystalline oxides is governed by the same bulk (or lattice diffusion) mechanisms as in metal single crystals. They proceed mainly via point defects (vacancies and interstices), leading to vacancy and interstitial diffusion. However, there are some differences in the defective structure of crystalline oxides compared to metals, ${ }^{246}$ with important implications for bulk diffusion:

- Crystalline oxides are predominantly ionic, consisting of at least two sublattices: a cation and an anion sub-lattice. The opposite charges of cations and anions induce diffusion always in the corresponding sub-lattice. Thus, cation diffusion occurs through the cation sub-lattice and anion diffusion occurs through the anion sub-lattice, as a difference with metals where diffusion is homogeneously occurring. ${ }^{246}$ 
- The interaction between crystal-forming particles in crystalline oxides is electrostatic and determined by their charges, sizes, and relative positions. Thus to analyze ionic motion, it is necessary to take into account the structure of an electric field, apart from geometrics only considered for metals. ${ }^{247}$

- The concentration of defects in oxides not only depends on the temperature or the total pressure as in metals, ${ }^{246}$ but also on the chemical potential of oxygen (controlled by the $\mathrm{O}_{2}$ partial pressure). However, in our diffusion studies presented in this thesis (Chapters 4 and 6), the concentration of defects can only be changed by temperature, since both total and $\mathrm{O}_{2}$ partial pressures are rather fixed, and cannot be manipulated, due to the experimental execution under atmospheric conditions.

- The crystal structure of the oxides significantly influences the defective structure. In the case of $\mathrm{RuO}_{2}$, it exhibits a rutile-like (body-centered tetragonal) structure with a hexagonal close packed (hcp) oxygen sub-lattice. ${ }^{14}$ Since defects in the oxygen sub-lattice have much higher enthalpies of formation due to this closer packing, ${ }^{246}$ they will be minor than in the cation sub-lattice, leading to slower diffusion of anions than cations in $\mathrm{RuO}_{2}$ (dominant cation diffusion). Other oxide structures with a more open oxygen sub-lattice would lead to an opposite situation. This is the case of $\mathrm{ZrO}_{2}$ where all its polymorphs (monoclinic, tetragonal and cubic) ${ }^{248}$ present a rather distorted (monoclinic and tetragonal) or non-distorted (cubic) fluorite-like structure, ${ }^{249}$ with a more open oxygen sub-lattice than the cation sub-lattice. ${ }^{246}$ The higher packing of the $\mathrm{Zr}^{4+}$ cation sub-lattice, reduces its amount of defects due to their higher enthalpy of formation. Thus, anion diffusion will be faster than cation diffusion in $\mathrm{ZrO}_{2}$ (dominant anion diffusion). ${ }^{246}$

- Stoichiometric oxides exhibit thermal disorder, and the concentration of defects is determined by intrinsic defect equilibria basically by simultaneous formation of vacancies in cation and anion sub-lattices (Schottky defect) or the formation of a pair ion vacancy-interstitial ion (Frenkel pair). As a difference, in metals there is only one lattice, and hence defects do not appear in pairs. ${ }^{247}$

- The incorporation of oxygen from the gas phase into the lattice or removal of oxygen from the lattice (extrinsic defects), will result in defects (Schottky or Frenkel pair) by non-stoichiometric oxide formation, with oxygen excess or deficit. This will lead to a different predominant anion or cation diffusion, depending on the non-stoichiometry type ${ }^{247}$ For instance, metal oxides with excess oxygen such as $\mathrm{RuO}_{\mathrm{x}}(2<\mathrm{x} \leq 3)$ present "extra" interstitial oxygen anions in the anion sub-lattice which will retard anion diffusion, and hence enhance cation diffusion. 
2. Polycrystalline oxides. Like in metals, apart from the mechanisms from bulk diffusion via lattice defects such as vacancies or interstices, in polycrystalline oxides there are grain boundaries, which are important short circuits for diffusion. It is known that at temperatures lower than half of the melting point $T_{m}$ (in K) of the material (the socalled Tamman temperature), ${ }^{250}$ diffusion through grain boundaries is much faster than bulk diffusion. Such that at temperatures below this Tamman temperature, grain boundary diffusion is the dominant process. It should be noted that sometimes Tamman temperature can be described as $0.7 T_{m} \cdot{ }^{251}$ For instance, considering that $\mathrm{RuO}_{2}$ directly sublimates upon annealing, with a sublimation point $T_{s}$ of $1200^{\circ} \mathrm{C},{ }^{38}$ a Tamman temperature value of $\sim 463^{\circ} \mathrm{C}\left(0.5 T_{s}\right)$ can be obtained. It means that at temperatures below this value, diffusion through $\mathrm{RuO}_{2}$ seems to undergo predominantly via grain boundaries. Since our diffusion studies of "oxygen" through $\mathrm{RuO}_{2}$ thin films presented in this thesis (Chapter 4) are performed at temperatures below this value, grain boundary diffusion might be the main mechanism, when considering the polycrystalline structure of the $\mathrm{RuO}_{2}$ films. The existence of a grain boundary mechanism could be confirmed by means of analysis techniques such as atomic probe tomography. However, it goes beyond the scope of this thesis.

3. Amorphous oxides. Amorphous metal oxides (AMOs) , as part of amorphous solids, exhibit a lack of the long-range ordering characteristic of crystalline materials. However, there is still some short-range ordering consisting of the arrangement of the nearest-neighbor ion that configures their microstructure, and strongly depends on the material. For instance, amorphous $\mathrm{ZrO}_{2}\left(\mathrm{a}-\mathrm{ZrO}_{2}\right)$ exhibits a predominant seven- and sixfold-coordination of the $\mathrm{Zr}^{4+}$ cations. ${ }^{252}$ Even there is some sort of short-range ordering and prevalent coordination in AMOs, their general structure is rather complex, random, and similar to a "glassy material". One important feature is that they usually do not present diffusion short circuits such as grain boundaries, and diffusion through them is limited to similar mechanisms, as the ones described for bulk diffusion in monocrystalline oxides (via interstitials or vacancies). This means that structural defects in the amorphous "lattice" will be important and dominating the diffusion process. However, since these defects might be randomly generated and difficult to predict, a way to study the defective structure of amorphous oxides is by studying their stoichiometry and, especially, their mass density. This has been studied in this thesis for $\mathrm{ZrO}_{2}$ thin films grown by two deposition conditions on a-Si (Chapter 6). There the mass density and the stoichiometry are evaluated and suggested to influence their defective structure, and hence, the oxygen diffusion. It should be noted that in this study oxygen diffusion is evaluated indirectly by the oxidation of the underneath a-Si layer, which means that the interfacial compound might also influence and, even limit, the diffusion process. Another concern for this study is the crystalline structure of $\mathrm{ZrO}_{2}$ films, which has been investigated in Chapter 6. An "XRD amorphous phase" has been detected for $\sim 2 \mathrm{~nm}$ low$\mathrm{O} \mathrm{ZrO}_{2}$ on a-Si samples, without appreciable signs of crystallinity. 
Temperature. The effective diffusion rate $D_{\text {eff }}$ is strongly influenced by the temperature (energy source), such that at higher temperatures the mobility of the diffusing species is enhanced, and additional unavoidable defects can be generated, usually leading to larger $D_{e f f}$, and hence faster diffusion. Normally the dependence of $D_{e f f}$ on the temperature $T$ in diffusion-limited growth of oxide thin films, as in many thermally activated processes, ${ }^{253}$ can be described by the Arrhenius equation (Eq. (4.2)), as suggested for $\mathrm{RuO}_{2}$ thin film growth on Chapter 4 (where $D_{\text {eff }}$ is renamed as $D$ ). If the process follows Arrhenius behavior, from plotting $\ln D$ against $1 / k_{B} T$ it is possible to obtain the activation energy $E_{a}$ and the pre-exponential factor $D_{0}$, as described in Chapter 4. Apart from the exponential dependence of $D$ (or $D_{\text {eff }}$ ) on the activation energy $E_{a}$ predicted by Arrhenius, in some thermally activated processes, the pre-exponential factor $D_{0}$ might also depend exponentially on the activation energy $E_{a}$ as:

$$
D_{0}=D_{00} e^{\frac{E_{a}}{E_{M N}}, 254}
$$

where $D_{00}$ and $E_{M N}$ (the so-called Meyer-Neldel energy) are constants independent of the temperature. This relationship is known as the empirical Meyer-Neldel rule (MNR). ${ }^{255}$ The physical interpretation of $D_{00}$ and $E_{M N}$ is not yet clear, and it has been subjected to different speculations. ${ }^{254,256,257}$ One general explanation to this rule can be associated to the proportionality between the entropy change $\Delta S$, and the activation energy $E_{a}$ (this last associated to the enthalpy change $\Delta H$ ), to achieve the activated state. ${ }^{258}$ When applying this general explanation to solid state diffusion, which usually involves multiphonon processes, this rule can be interpreted as the increase of the number of paths to reach the activated state with increasing activation energy $E_{a} \cdot{ }^{259}$ This is due to the fact that $\Delta S$ is proportional to the logarithm of the number of paths of assembling the many excitations or the multiphonon processes. ${ }^{260}$ Another explanation to this rule, is that multiple independent processes might contribute to the overall diffusion process $(D)$, where an intrinsic transport process (for instance, by metal cations or oxygen anions) is the main contribution, and other mediated processes, such as amount of annealing or diffusion of impurities, are the minor contribution. ${ }^{254}$ This last minor contribution results in a small activation energy, the Meyer-Neldel energy $E_{M N}$, which in solid diffusion normally exhibits values between 25 to $75 \mathrm{meV} .{ }^{261}$ For instance, in our "oxygen" diffusion study through $\mathrm{RuO}_{2}$ which seems to obey the Meyer-Neldel behavior, a value of $\sim 60 \mathrm{meV}$ is obtained, as presented in this thesis (Chapter 4).

\subsubsection{Current status of Ru thin films oxidation: diffusion-limited growth case}

Oxidation of monocrystalline $\mathrm{Ru}$ has been extensively investigated during the last decades. ${ }^{219,230,262,263,264}$ Recently, oxide thin films and 3D islands has been observed during thermal oxidation of single crystalline $\mathrm{Ru} .{ }^{223,224}$ However, for polycrystalline $\mathrm{Ru}$, no $3 \mathrm{D}$ islands have been seen, with only $2 \mathrm{D}$ thin film oxide growth being 
observed. ${ }^{265,266,267}$ Although, an increased roughening has been detected at enhanced temperatures in these studies, ${ }^{267}$ it has not been recognized as 3D growth, and only thin film $\mathrm{RuO}_{\mathrm{x}}$ growth has been reported. ${ }^{265,266}$ In this thesis (Chapter 3), we report on the simultaneous 2D (thin film) and 3D (nano-column) growth of ruthenium oxide, experimentally observed for thermally oxidized polycrystalline $\mathrm{Ru}$ thin films. In addition, it is also found that the thin film oxide does not grow as a single layer but a combination of two layers on top of each other, a low density $\mathrm{RuO}_{\mathrm{x}}(2<\mathrm{x} \leq 3)$ layer on top of a near bulk density $\mathrm{RuO}_{2}$ layer. As stated in the previous section (Sec. 1.4.3.1), oxidation of polycrystalline $\mathrm{Ru}$ is relevant for microelectronic (DRAM, CMOS) devices and EUVL optics, since $\mathrm{Ru}$ is used as $\mathrm{Cu}$ diffusion barrier and/or $\mathrm{Cu}$ seed layer in integrated circuits with copper interconnect technology, ${ }^{4}$ as bottom electrode in capacitors based on high dielectric materials, ${ }^{5,6}$ or as capping layer for EUVL optics, $7,8,9,10$ with a similar polycrystalline (or amorphous) structure far from monocrystalline. ${ }^{234}$

\subsubsection{Current status of oxygen diffusion through $\mathrm{RuO}_{2}$ and $\mathrm{ZrO}_{2}$ films}

Oxidation of monocrystalline and polycrystalline $\mathrm{Ru}$ has been extensively studied in literature, ${ }^{219,230,265}$ including monitoring the growth of $2 \mathrm{D}$ thin film oxide in time. ${ }^{266,267}$ However, in all these studies which monitor the oxide growth, there is no either experimental or simulated data in diffusion kinetics (diffusion constants and activation energies) for oxygen diffusion in $\mathrm{RuO}_{2}$ thin films that would allow to understand how oxygen penetrates a $\mathrm{RuO}_{2}$ protective film towards underneath layers during thermal oxidation. Thus, in this thesis (Chapter 4), the diffusion rates and activation energies for oxygen diffusion through a few nanometer thick ruthenium oxide layer have been accurately determined. This ruthenium oxide layer consists of two separate layers: a saturated low density amorphous $\mathrm{RuO}_{\mathrm{x}}(2<\mathrm{x} \leq 3)$ layer on top of a growing high density polycrystalline $\mathrm{RuO}_{2}$ layer (see Chapter 3). Thus, oxygen diffusion has been stablished through this $\mathrm{RuO}_{\mathrm{x}} / \mathrm{RuO}_{2}$ structure (Chapter 4). It should be noted that diffusion combines $\mathrm{Ru}^{4+}$ cations and $\mathrm{O}^{2-}$ anions diffusion, but the overall diffusion process can be expressed by an effective "oxygen" diffusion rate, with an associated activation energy.

There are some studies of oxygen diffusion through amorphous $\mathrm{ZrO}_{2}$ thin films during thermal oxidation. ${ }^{62,268} \mathrm{~A}$ transition from a- $\mathrm{ZrO}_{2}$ to $\mathrm{t}-\mathrm{ZrO}_{2}$ has been reported at different temperatures depending on the $\mathrm{ZrO}_{2}$ thickness, enhancing the oxygen diffusion above this temperature due to crystallization (possible grain boundary diffusion). For instance, for a $\sim 7-\mathrm{nm}$ - and $\sim 13$-nm-thick $\mathrm{ZrO}_{2}$ sample, this crystallization temperature has been found to be $\sim 600^{\circ} \mathrm{C}$ and $400^{\circ} \mathrm{C}$, respectively. ${ }^{62}$ However, these $\mathrm{ZrO}_{2}$ layer thicknesses are larger than the typical thicknesses (normally $<5$ nanometers) required for dielectric layers in CMOS and memory devices, passivation layers in solar cells, or capping layers for EUVL optics. In addition, $\mathrm{ZrO}_{2}$ layers have been deposited on natively 
oxidized or HF-cleaned $\mathrm{Si}(100),{ }^{62,268}$ and not directly on a-Si (the typical ending layer for a-Si solar cells or EUVL mirrors). Such that in this thesis (Chapter 6), we have studied oxygen diffusion through a $\sim 2 \mathrm{~nm}$ a- $\mathrm{ZrO}_{2}$ layer on a-Si during thermal oxidation. It should be noted that upon deposition a $\mathrm{ZrSiO}_{4}$ interlayer compound forms. Hence, the oxygen diffusion process is through the $\mathrm{ZrO}_{2} / \mathrm{ZrSiO}_{4}$ structure. Although a temperature $>400^{\circ} \mathrm{C}$ is observed in Chapter 6, where oxygen diffusion seems to increase, it would be difficult to associate it to a crystallization process, as in ref. ${ }^{62}$, due to the large $(\sim 2.6 \mathrm{~nm})$ $\mathrm{ZrO}_{2} / \mathrm{Si}$ intermixing, already upon deposition. However, the nature of this enhanced diffusion has not been investigated in Chapter 6 , requiring further research to elucidate its causes.

\subsection{Outline of this thesis}

Oxidation of underneath layers is one of the problems in the fabrication of microelectronics (CMOS and memory devices), photovoltaics, and also during their operation (including the EUVL optics operation), since it reduces the capacitance of the electronics, the UV transmission and photo-generated power of the solar cells, and the reflectance of the EUVL optics, decreasing their lifetime. A lot of research has been done focused on the development and use of oxidation resistant layers for protecting these devices against oxidation, ${ }^{22,34,36,40,45,113}$ as discussed in Sec. 1.2.3. However, in all these studies there is a lack of understanding about the processes involved during growth and oxidation, and oxidation (or oxygen diffusion) kinetics of these protective layers. Thus, this thesis gives further insights into the growth (processes, phenomena) and oxidation (mechanisms, diffusion kinetics) of $\mathrm{Ru}$ and $\mathrm{ZrO}_{2}$ thin films used as oxidation resistant protective layers for DRAMs, CMOS devices, solar cells and EUVL optics. As discussed in Secs. 1.3.1 and 1.4.1.1, room-temperature magnetron sputtering and thermal oxidation have been selected among the other deposition and oxidation techniques for growing and oxidizing these thin films. The particular sputtering deposition and thermal oxidation setups used are presented in Chapter 2. In addition, all analysis techniques used to investigate the growth and thermal oxidation of $\mathrm{Ru}$ and $\mathrm{ZrO}_{2}$ thin films are also discussed in Chapter 2.

In the framework of microelectronics, photovoltaics and EUVL optics, polycrystalline (or amorphous) thin films are typically applied as protective materials for these devices. Although monocrystalline materials are usually not applied, still similar oxidation phenomena might occur in polycrystalline (or amorphous) materials compared to monocrystalline, which makes it worth to investigate. In monocrystalline $\mathrm{Ru}$, there has been a lot of research in thermal oxidation. ${ }^{219,230,262}$ Recently, thin film and 3D island oxide growth have been observed for this system. ${ }^{223,224}$ Contrarily, for polycrystalline $\mathrm{Ru}$, no 3D islands have been detected, with only thin film oxide growth being observed. ${ }^{265,266}$ Combining XRR, AES, AR-XPS, AFM, XRD and HR-TEM, a 
concurrent 2D and 3D ruthenium oxide growth model has been proposed in Chapter 3 for thermal oxidation of polycrystalline Ru thin films. In addition, in situ XRR has also allowed us to detect that the thin film (2D) ruthenium oxide does not grow as a single layer but a combination of two layers on top of each other, a low density $\mathrm{RuO}_{\mathrm{x}}(2<\mathrm{x} \leq 3)$ layer on top of a near bulk density $\mathrm{RuO}_{2}$ layer, as also presented in Chapter 3 .

There is a lack of diffusion rates and activation energies for oxygen diffusion in polycrystalline $\mathrm{RuO}_{2}$ thin films. This is due to the difficult measurement of diffusion coefficients in systems with short diffusion lengths. ${ }^{269}$ The typical techniques for this purpose such as TEM or XPS, do not allow to determine small changes in the structures with sufficient precision. To tackle this problem, in situ XRR measurements are used in Chapter 4 for an accurate determination of the in-depth electron density distribution during thin film ruthenium oxide growth. From this information, diffusion rates and activation energies for oxygen diffusion through polycrystalline ruthenium oxide films are also accurately obtained in Chapter 4.

There is a limited amount of techniques that allow in situ monitoring growth, including the determination of the thickness when the layer closes with Ångström precision. From these techniques, LEIS is considered to be unique for such studies. ${ }^{270}$ It also gives information about the intermixing with the underneath material. Both features are relevant for studying relevant protective layers for microelectronics, photovoltaics and EUVL optics. In the case of Ru protective layer, there are few studies reported for $\mathrm{Ru}$ grown on $\mathrm{Si}^{183}$ But there are no reported growth studies at room temperature for $\mathrm{Ru}$ grown on a-Si, which is usually the last layer of, for instance, a-Si solar cells or EUVL multilayer mirrors. Thus, high-sensitivity LEIS has been used in Chapter 5 for in vacuo monitoring Ru growth on a-Si. To study the influence of the passivation (by nitridation or oxidation) of this a-Si layer, $\mathrm{Ru}$ has been also grown on $\mathrm{SiN}$ and $\mathrm{SiO}_{2}$ substrate layers. Hence, $\mathrm{Ru}$ surface coverages and thicknesses required for closing the Ru layer on all three substrates are presented. From the $\mathrm{Ru}$ surface coverages, the in-depth $\mathrm{Ru}$ concentration profiles for these systems are also reconstructed in Chapter 5. In addition, the growth of the reverse system ( $\mathrm{Si}$ on $\mathrm{Ru}$ ) is also investigated, which allows to distinguish the different contributions to the intermixing between $\mathrm{Ru}$ and $\mathrm{Si}$.

In Chapter 6, we investigate $\mathrm{ZrO}_{2}$ as an alternative capping layer to $\mathrm{Ru}$ to protect DRAMs, CMOS devices, solar cells and EUVL optics against oxidation. In particular, in vacuo high-sensitivity LEIS has been used in this chapter to study the initial stages of growth of reactive sputtered $\mathrm{ZrO}_{2}$ thin films on a-Si for two gas deposition conditions, with high and low oxygen content, mimicking the two oxidic and metallic sputtering modes from ref. ${ }^{157}$. Similar to $\mathrm{Ru}, \mathrm{ZrO}_{2}$ has also been grown on $\mathrm{SiN}$ and $\mathrm{SiO}_{2}$ substrate layers. In addition, in vacuo XPS is used in Chapter 6 to determine the $\mathrm{ZrO}_{2}$ stoichiometry and its initial interaction with the underneath a-Si layer for both deposition 
Chapter 1

conditions. Furthermore, ex-situ AR-XPS is used to investigate the oxygen diffusion response upon thermal oxidation of the $\mathrm{ZrO}_{2} / \mathrm{Si}$ system.

Finally, the valorization and outlook of this thesis is presented in Chapter 7. 


\section{Experimental}

\subsection{Sputter deposition setups}

All thin films investigated in this thesis have been deposited by room temperature DC magnetron sputtering. The two sputter deposition setups used in this thesis for growing the films are the ones located at the XUV Optics lab of the Industrial Focus Group XUV Optics from the MESA+ Institute for Nanotechnology (University of Twente), and are described as follows.

\subsubsection{Advanced Development Coater (ADC)}

ADC is an ultra-high vacuum (UHV) deposition setup with a base pressure of $<10^{-8}$ mbar, equipped with six e-beam evaporators, four magnetron sputtering sources and one Kaufman ion gun. The schematic diagram of this setup is depicted in Fig. 2.1. For magnetron deposition, the magnetron is moved to the central position facing the sample substrate attached to the holder. The holder is constantly rotated to ensure homogeneous deposition across the radial direction, allowing multiple sample copies mounted along this direction. The shutter regulates when the deposition takes places, by blocking or allowing the particle flux arriving at the sample substrate from the magnetron target. The distance between the target and the substrate holder is $\sim 30 \mathrm{~cm}$, larger than typical magnetron sputtering setups. This distance combined with an increased sputter gas pressure, allows reduced surface damage during deposition. The layer thickness is monitored during deposition by quartz mass balances, previously calibrated by ex situ $\mathrm{X}$-ray reflectivity, as later discussed in Sec. 2.3.1. ADC is the coater used for deposition of the layered samples in Chapters 3 and 4. More information of the sample and deposition conditions can be found in each chapter. 


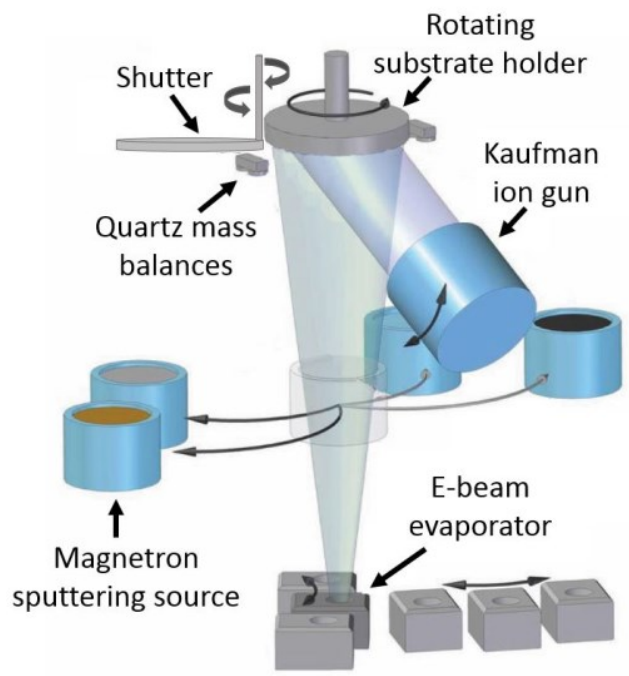

Fig. 2.1. Schematic diagram of the ADC coating facility.

\subsubsection{Atomic Growth and Analysis (AGA)}

AGA is an UHV coater with a base pressure of $<2 \times 10^{-10}$ mbar, consisting of six magnetron sputtering sources and one Kaufman ion gun. The schematic diagram of AGA is shown in Fig. 2.2. In this case, the magnetrons are fixed and the substrate holder is transported to the required position. Contrarily to ADC, AGA allows only one sample coating at the time, since the holder only can handle one substrate. In this case, the targetsubstrate distance is $\sim 10 \mathrm{~cm}$, within the usual distance in magnetron sputtering. ${ }^{271}$ Similarly, the layer thickness is monitored during growth by calibrated quartz mass balances (see Sec. 2.3.1). AGA is the coater used for the deposition of the layered samples in Chapters 5 and 6. More information of the sample and deposition conditions can be found in each chapter.

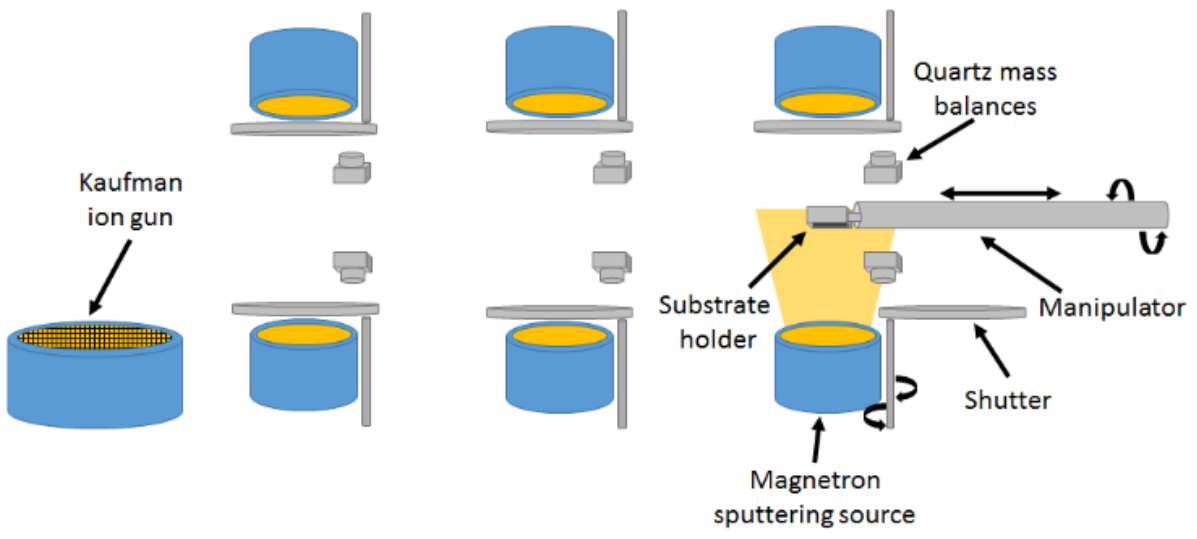

Fig. 2.2. Schematic diagram of the AGA coating facility. 
AGA is part of a combined deposition and analysis cluster, which allows in vacuo analysis just after deposition without atmospheric exposure (see image and scheme of the cluster setup in Fig. 2.3 (a), and (b), respectively). This cluster maintains an UHV ( $\sim 2 \times 10^{-10}$ mbar) atmosphere during the sample transfer ( $\left.\sim 5 \mathrm{~min}\right)$ from AGA to one of the two connected surface analysis techniques: low energy ion scattering (LEIS) or Xray photoelectron spectroscopy (XPS) (see description in Secs. 2.3.2 and 2.3.3, respectively). It should be noted that in the case of LEIS analysis, the short measurement time ( 2 min) ensures an "uncontaminated" sample state, while the longer XPS analysis time $(\sim 1 \mathrm{hr})$ allows a minor surface contamination $(<1 \mathrm{ML}),{ }^{211}$ collected during the measurement.
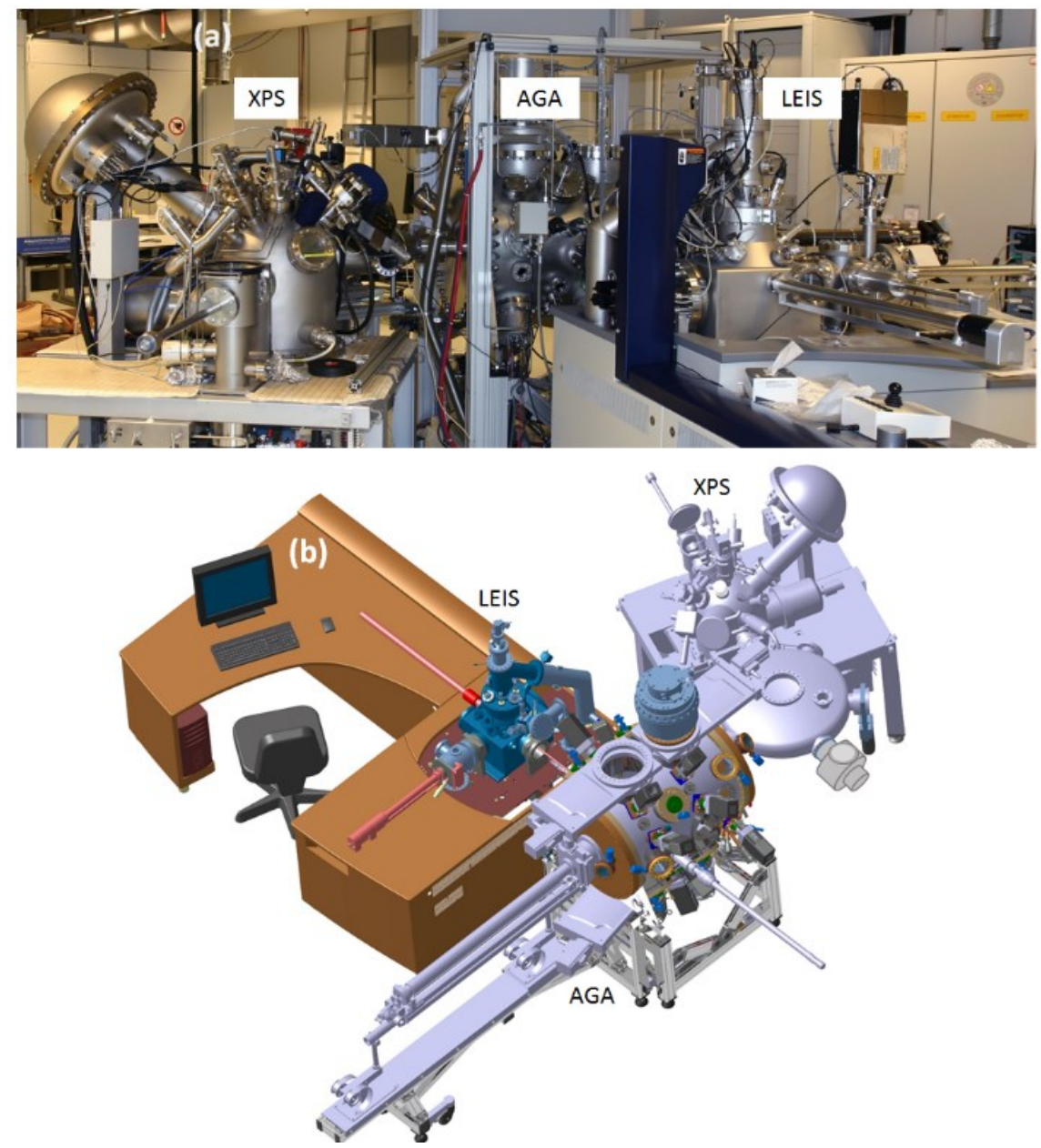

Fig. 2.3. Image (a), and scheme (b) of the deposition and analysis cluster setup. 


\subsection{Thermal oxidation setups}

Due to the complexity of EUV induced processes, and moreover, due to the difficult access to EUV sources to carry out such experiments, the oxidation studies presented in this thesis have been performed using controlled lab based thermal oxidation setups. Although the processes leading to oxidation may differ depending on the oxidation source, the processes that take place when oxidation continues in depth, will show great similarities (diffusion kinetics and link to structure), and validate the choice for the lab based thermal oxidation setups presented as follows.

\subsubsection{DHS 1100 Domed Hot stage}

This annealing setup from Anton Paar consists of a ceramic heating stage located under a dome-shaped made of graphite, as shown in Fig. 2.4 (b). The stage can reach temperatures up to $1100^{\circ} \mathrm{C}$ by resistive heating, with a stability and reproducibility within $0.5^{\circ} \mathrm{C}$. Due to variations in thermal conductivity, the temperature on top of the sample can deviate by few ${ }^{\circ} \mathrm{C}\left(<2^{\circ} \mathrm{C}\right.$, for temperatures below $\left.400^{\circ} \mathrm{C}\right)$. Although higher temperatures can lead to larger deviations, still these deviations can be considered negligible, allowing reproducibility for the thermal oxidation studies of polycrystalline $\mathrm{Ru}$ conducted in this thesis (Chapters 3 and 4). A more detailed description of this annealing setup can be found in refs. ${ }^{272,273}$. This setup is integrated into an X-ray diffractometer for in situ XRR and XRD measurements, allowing in situ monitoring of structural changes during annealing, as described later in Secs. 2.3.5 and 2.3.6.
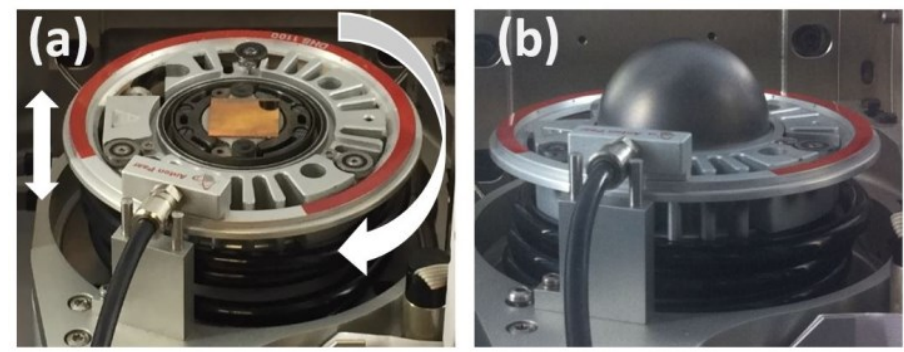

Fig. 2.4. Images of the DHS 1100 hot stage setup with a $25 \times 25 \mathrm{~mm}$ sample mounted on the top, without the dome (a) and with the dome (b).

\subsubsection{Elf 11/14 laboratory furnace}

To enable annealing of sample batches with a controllable and reproducible temperature within $\sim 5^{\circ} \mathrm{C}$, a CARBOLITE Elf $11 / 14$ furnace is used. This furnace can achieve temperatures up to $1100^{\circ} \mathrm{C}$, by several free radiating wire elements embedded in the chamber sides, equipped with a temperature controller with a single ramp to set-point. This setup is used in this thesis for the thermal oxidation studies of $\mathrm{ZrO}_{2}$ thin films on aSi (Chapter 6). 


\subsection{Analysis and characterization techniques}

The analysis and characterization techniques used in this thesis for studying the growth and thermal oxidation of $\mathrm{Ru}$ and $\mathrm{ZrO}_{2}$ thin films are discussed as follows.

\subsubsection{Quartz mass balances}

During thin film deposition, both deposition rate and layer thickness are monitored by off-centered quartz mass balances (QMBs) mounted inside each deposition setup. An image of four quartz mass balances installed in the ADC coating facility below the rotating substrate holder before operation, and an image of one quartz mass balance installed in the AGA coating facility on top of one of the magnetron sources during operation are displayed in Fig. 2.5 (a) and (b), respectively.
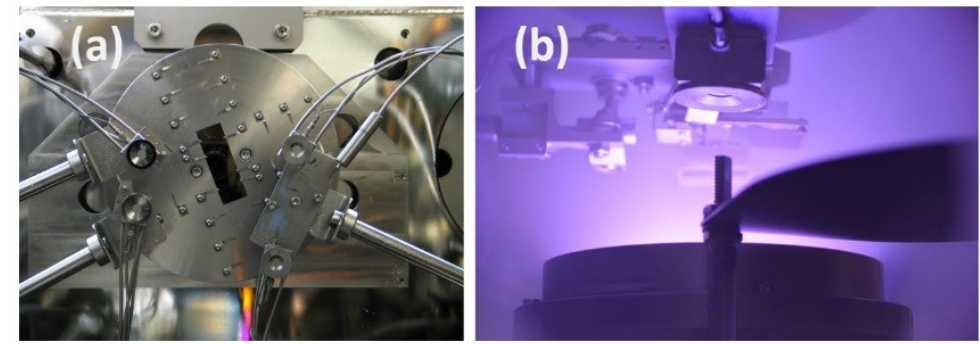

Fig. 2.5. Image of the $A D C$ rotating substrate holder with three uncoated Si substrates and four quartz mass balances on the sides before operation (a, bottom view), and image of the AGA manipulator with a substrate holder and one quartz mass balance facing a magnetron source during operation (b, side view).

The operating principle of the QMB is based on the frequency change of their integrated quartz crystal resonator during film deposition. The deposited layer thickness can be determined with an accuracy of $\sim 0.3 \AA$, limited basically by temperature variations. Due to their off-center position, an accurate estimation of the deposited thickness in the substrate center can only be obtained by external (X-ray reflectivity based) calibration of the QMB. Due to the high stability and reproducibility of the magnetron sputter sources in the ADC coating facility, their thickness control, ( few \%) in this thesis, is performed by calibrated deposition rates, while QMB thicknesses are monitored to recognize process interruptions. Due to small variations of the plasma conditions and deposition rates in the AGA coating facility, the control of the deposited thickness is performed directly by measured QMB thickness, after applying the proper calibration factors, leading to $\sim 5 \%$ reproducibility. The thickness errors introduced by both deposition setups are well below the level needed to reproduce the layered structures for the research presented in this thesis. 


\subsubsection{Low-energy ion scattering}

\subsubsection{Motivation for its use}

There is a limited availability of techniques for in situ monitoring initial thin film growth in the sub- and nanometer scale. Especially, an accurate determination of the thickness where the layer closes has turned out to be a problem. ${ }^{274,275}$ Short diffusion lengths involved, necessity of ultra-high vacuum tools, possible matrix effects or variations in morphology and interlayer roughness/intermixing during growth, the need for high-precision real-time measurements and compatibility with amorphous materials are some of the main difficulties to overcome.

From all surface analysis techniques, low energy ion scattering (LEIS) can overcome most of these issues, and is considered to be unique for studying the initial stages of thin film growth. This uniqueness relies on the capacity of LEIS to fully separate the information of the outermost surface atomic layer from the layers below the surface, ${ }^{270,276}$ providing an accurate monitoring of the surface composition during growth of a thin film until it closes.

\subsubsection{LEIS principles}

LEIS is a surface analysis technique in which noble gas ions $\left(\mathrm{He}^{+}, \mathrm{Ne}^{+}\right.$or $\left.\mathrm{Ar}^{+}\right)$are directed towards the sample (target) with ion-energies between 0.5 and $10 \mathrm{keV}$ and an incident angle $\alpha$ with respect to the surface normal $<60^{\circ}$. According to kinematics (momentum and energy conservation laws), when considering an elastic binary collision of an incoming ion with mass $m_{1}$ and kinetic energy $E_{0}$ with a target atom with mass $m_{2}$ at rest, the following expression for the energy of the scattered ion $E_{f}$ is obtained:

$$
E_{f}=\left[\frac{\cos \theta+\sqrt{\left(\frac{m_{2}}{m_{1}}\right)^{2}-\sin ^{2} \theta}}{1+\frac{m_{2}}{m_{1}}}\right]^{2} \cdot E_{0},
$$

where $\theta$ is the scattering angle. It should be noted that this equation is only valid for $m_{2}>m_{l}$ and for $\theta \geq 90^{\circ} .270$

According to Eq. (2.1), for an incident ion energy $E_{0}$ and ion mass $m_{l}$ and a fixed scattering angle $\theta$, the scattering energy $E_{f}$ only depends on the mass of the surface atom $m_{2}$, and hence, by measuring $E_{f}$ from 0 to $E_{0}$ values on a sample surface it is possible to determine the surface composition of the sample. The elastic binary collision at the surface is represented by the position of the surface peaks (certain $E_{f}$ ) present in the LEIS spectrum, where each peak corresponds to a certain mass $m_{2}$, and thus to a certain element. For instance, the scattering of $3 \mathrm{keV} \mathrm{He}^{+}$ions on a bare $\mathrm{Ru}$ sample, considering a fixed scattering angle $\theta$ of $145^{\circ}$ (used in our setup), leads to a scattering energy $E_{f}$ of 
$2597 \mathrm{eV}$ when using Eq. (2.1). This means that ideally only one peak centered at 2597 $\mathrm{eV}$ corresponding to $\mathrm{Ru}$ will be present in the spectrum. However, due to small inelastic losses, the peak is shifted to lower energies, as observed in the LEIS spectrum presented for the $4.7 \mathrm{~nm} \mathrm{Ru}$ sample in Chapter 5 (Fig. 5.2). There, the Ru peak is centered at $\sim 2500$ $\mathrm{eV}$. Regardless of this inelastic shift, each surface element (when $m_{2}>m_{1}$ ) can be identified with a characteristic surface peak with a characteristic $E_{f}$ associated, and the intensity of each surface peak is proportional to the surface concentration (or coverage) of the corresponding element. ${ }^{276}$

Apart from the surface composition provided by the surface peaks, the sub-surface composition ( $\sim 0$ to $10 \mathrm{~nm}$ in-depth concentration profile, depending on the energy and target material) can be experimentally determined from the energy distribution of the reionized neutrals scattered below the surface. This energy distribution is represented by the background at energies lower than the surface peak ("tail" to the peak) in the LEIS spectrum (see "low-energy tail" of Ru for the $4.7 \mathrm{~nm}$ sample in Fig. 5.2 in Chapter 5). The re-ionized neutrals carry less energy than projectiles scattered at the surface due to (inelastic) collision processes. ${ }^{276}$ The energy loss $<\Delta E>$ is obtained by:

$$
<\Delta E>=\int S(E, x) d x
$$

where $S$ is the (energy- and material-dependent) stopping power, and $x$ the travel distance of the projectile. A typical assumption is to consider the stopping power $S$ to be energyindependent. With this assumption and considering the scattering geometry (see Fig. 2.6), Eq. (2.2) can be easily integrated as:

$$
<\Delta E=S d\left(\frac{1}{\cos \alpha}-\frac{1}{\cos (\alpha+\theta)}\right),
$$

where $d$ is the depth at which the projectile is backscattered. For our LEIS system (where $\alpha=0^{\circ}$ and $\theta=145^{\circ}$ ), Eq. (2.3) becomes Eq. (5.6). Considering Eq. (5.6), for a certain $<\Delta E>$, when knowing the stopping power $S$, it is possible to determine the depth $d$ at which the projectile is backscattered in our specific LEIS setup. This is used in Chapter 5 to determine the maximum depth $d$ where the deepest Ru or Si atom is located for each deposited $\mathrm{Ru}$ or Si thickness. By plotting the surface coverages (for each deposited thickness) as a function of this determined $d$ values, the respective in-depth $\mathrm{Ru}$ or $\mathrm{Si}$ "effective" concentration profiles can be obtained, as described in detail in Chapter 5. 


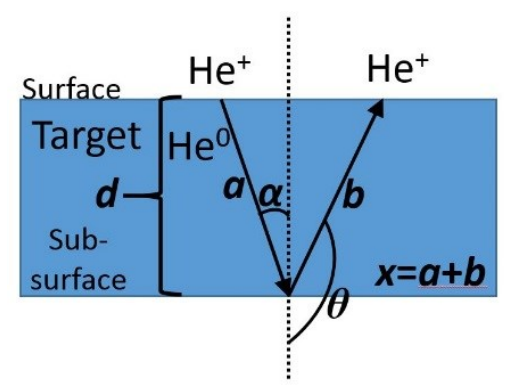

Fig. 2.6. Schematic diagram of sub-surface scattering of a He projectile from a target. The backscattering depth $d$, the travel distance $x(x=a+b)$, the incident angle $\alpha$, and the scattering angle $\theta$ are indicated. It should be noted that the $\mathrm{He}^{+}$ion becomes $\mathrm{He}^{0}$ neutral when entering the surface, travels as neutral through the sub-surface, and re-ionizes again after reaching the surface.

\subsubsection{High-sensitivity LEIS setup}

LEIS analyzers have been improved over the last three decades by Brongersma et al., ${ }^{277}$ and a new double toroidal analyzer (DTA) has been developed. This analyzer has been incorporated into an advanced commercial LEIS equipment released in 2008 by ION-TOF GmbH. ${ }^{278}$ The so-called high-sensitivity low-energy ion scattering (HSLEIS), ${ }^{279}$ under the commercial name of $\mathrm{Q}_{\mathrm{tac}}{ }^{100}$, which requires very low ion fluency for the measurement, enables the "static" analysis with negligible damage from the probing ions, and has resulted in better detection limits for the surface elements. For instance, the detection limit for the surface coverage for lighter elements (from $\mathrm{Li}$ to $\mathrm{O}$ ) is $\geq 1 \%$ and for heavier elements (from K-U) is between 500 to 10 ppm. ${ }^{276}$ This HS-LEIS setup $\left(\mathrm{Q}_{\mathrm{tac}}{ }^{100}\right)$ connected in vacuo with a sputter deposition setup (AGA) is the one used in this thesis for "in situ" monitoring of the initial growth of $\mathrm{Ru}$ and $\mathrm{ZrO}_{2}$ thin films (Chapters 5 and 6 , respectively), including an accurate determination of the surface coverages and the thicknesses required for closing the layer on a-Si, $\mathrm{SiN}$ and $\mathrm{SiO}_{2}$ substrates.

\subsubsection{X-ray photoelectron spectroscopy}

\subsubsection{Motivation for its use}

XPS provides in addition to the atomic composition, the chemical (bonds) composition of the surface and near sub-surface of the sample. ${ }^{280}$ However, in contrast with LEIS, which especially and quantitatively provides the atomic composition of the outermost atomic layer, due to the photoelectron mean free path of several nanometers, XPS averages the composition over the outer 5-20 atomic layers. ${ }^{276}$ Although it averages over the few top layers, XPS is very useful for determining the compound stoichiometry of thin film interfaces (such as silicides or silicates) or thin film multicomponent layers (such as $\mathrm{SiN}_{\mathrm{x}}, \mathrm{SiO}_{\mathrm{x}}$ or $\mathrm{ZrO}_{\mathrm{x}}$ ). Therefore, it has been used in this thesis in combination with a deposition setup for in vacuo monitoring the initial growth of $\mathrm{Ru}$ and $\mathrm{ZrO}_{2}$ 
protective layers, regarding the initial chemical interaction of these materials with the underneath substrate layer (Chapters 5 and 6, respectively). It also has been used to determine the compound stoichiometry of our $\mathrm{SiN}_{\mathrm{x}}$ and $\mathrm{SiO}_{\mathrm{x}}\left(\right.$ Chapter 5), and $\mathrm{ZrO}_{\mathrm{x}}$ (Chapter 6) reactive sputtered films for several sputtering gas mixtures. In addition, by means of angular-resolved XPS (AR-XPS), it is possible to discriminate the position (indepth) of the formed compounds (or elements) by providing a non-destructive in-depth atomic concentration profile, and to extract an effective thickness of usually one or two overlayer compounds (or elements) on a substrate. ${ }^{281,282}$ Therefore, AR-XPS is used in this thesis for ex situ studying the stoichiometry and the in-depth distribution of ruthenium oxides during thermal oxidation (Chapter 3), and oxygen diffusion through $\mathrm{ZrO}_{2}$ thin films on a-Si (Chapter 6).

\subsubsection{XPS principles}

XPS irradiates the sample with monochromatic soft X-ray radiation with a photon energy $h v$. This energy is absorbed by the sample emitting core electrons (photoelectrons) of which their original binding energy $E_{b}$ can be determined by:

$$
E_{b}=h v-E_{k}-\Phi
$$

where $E_{k}$ is the kinetic energy of the emitted photoelectron, and $\Phi$ is the work function, dependent on both the spectrometer and the material. Since $h v$ and $\Phi$ are known parameters, $E_{b}$ can be determined directly by measuring the emitted photoelectrons as function of $E_{k}$, according to Eq. (2.4). There are several characteristic XPS peaks corresponding to different orbitals for each element with a characteristic binding energy associated. Thus, from these characteristic peaks in the XPS spectrum, it is possible to identify the sample elements and their atomic concentrations. In addition, binding energy shifts due to the local environment of the sample atoms, allow to determine their chemical states. $^{280}$

\subsubsection{XPS setup}

A Thermo Theta Probe spectrometer using monochromatic Al-K $\alpha$ radiation $(\mathrm{hv}=$ $1486.6 \mathrm{eV})$ is the XPS setup used in this thesis. The resolution of this setup is restricted to the size of the smallest analysis area $(\sim 15 \mu \mathrm{m})$. However, for the quantitative analysis presented in this thesis, the analysis area, and hence the resolution, is limited to $\sim 200$ to $400 \mu \mathrm{m}$. 


\subsubsection{Auger electron spectroscopy}

\subsubsection{Motivation for its use}

Similar to XPS, Auger electron spectroscopy (AES) is a surface analysis techniques that provides the atomic composition of the surface and near sub-surface of a sample. ${ }^{280}$ In contrast to XPS, its probing depth is lower (from $\sim 1$ to $3 \mathrm{~nm}$ ), giving more information of the surface region. Also AES can typically achieve a higher lateral resolution $(<1$ $\mu \mathrm{m}$ ), while the spatial resolution of XPS is mostly limited to $\sim 15 \mu \mathrm{m}$. Quantitative information of the atomic surface composition by AES is much limited and difficult to obtain than by XPS, due to the fact that the AES signal strongly depends on the sample surface morphology. In some cases, the chemical state of the surface atoms can also be determined by AES, from the energy shits and peak shapes, but it is also limited and difficult to obtain when compared to XPS. Therefore, AES is used locally in this thesis (Chapter 3) to qualitatively study the surface atomic composition of our oxidized $\mathrm{Ru}$ samples, where we can obtain separate surface elemental information from the columns and the areas between the columns. However, this analysis is limited by the AES resolution, and hence it can only be applied for the oxidized samples where the lateral sizes of the surface columns are larger than this resolution.

\subsubsection{AES principles}

AES uses an electron beam with a kinetic energy typically between 3 and $20 \mathrm{keV}$ to remove core shell electrons from target sample atoms, generating electron holes. These holes are filled by higher shell electrons in a relaxation process from ionized to ground state. This process releases energy which can be transferred to another electrons (the socalled Auger electrons) that are then emitted out of the sample with a certain kinetic energy $E_{k}$, and later detected by an electron analyzer. This kinetic energy $E_{\mathrm{k}}$ can be related to the orbital energies of the sample atoms by:

$$
E_{k}=E_{b}-E_{1}-E_{2},
$$

where $E_{b}, E_{1}$, and $E_{2}$ are the respective core level, first outer shell, and second outer shell electron binding energies. Since these binding energies of the electrons within atoms are element specific, the Auger electron kinetic energy $E_{k}$ is also element specific. Thus, by measuring the collected Auger electrons as function of their kinetic energy $E_{k}$, it is possible to obtain the surface atomic composition of the sample from the peaks in the AES spectrum. ${ }^{280}$ However, since AES peaks are mixed with a lot of background from primary and secondary electrons, the AES spectrum is usually derived, and the elements can be more easily identified and quantified by their respective peak positions and their peak-to-valley heights in the resulting spectrum. Finally, since AES often uses a focused electron beam, it can also be applied for either compositional mapping of the surface by 
scanning Auger (electron) microscopy (SAM) or topographical imaging of the surface by secondary electron microscopy (SEM) with sub- $\mu$ m resolution.

\subsubsection{AES setup}

The AES setup used in this thesis is the same as the XPS setup described in Sec. 2.3.3.3, since it incorporates a field emission electron source that allows AES, and also SAM and SEM. A kinetic energy of $5 \mathrm{keV}$ and a beam size of $\sim 1 \mu \mathrm{m}$ is used for the electron beam in both techniques. However, for AES, a point measurement (a relatively local probe) is performed in several position of the sample, whereas for SEM, the electron beam is scanned along a larger area of $\sim 400 \mu \mathrm{m}^{2}$ to create a SEM image of the sample.

\subsubsection{X-ray reflectivity}

\subsubsection{Motivation for its use}

To determine parameters of nanometer range thin films and dynamics of their growth accurately, an in situ technique needs to be applied. For instance, spectroscopic ellipsometry (SE) can be used for in situ monitoring thin film growth. ${ }^{283,284}$ However, it should be noted that extracting information from SE involves reconstruction of both optical constants and layered structure, a process which is often non-uniquely determined. (Specular) X-ray reflectivity (XRR) is another example of technique that can be used for in situ monitoring thin film growth. ${ }^{285}$ Although both SE and XRR require a model for extracting information, there is an important advantage of XRR compared to SE: XRR allows the reconstruction of the layered structure once the optical constants are provided with less correlation between optical and structural parameters. In contrast, SE presents difficulties for de-correlation between parameters, especially in the characterization of thin films with thicknesses $<10 \mathrm{~nm}$, due to its relative incapacity to discriminate between deposited and substrate layers. ${ }^{286}$ Since optical constants in the Xray region are usually known for most elements or compounds (experimentally or by calculations), ${ }^{287}$ XRR allows an accurate reconstruction of the layered structure: thicknesses, densities and roughness's of the different layers with accuracies of about $\pm 0.1 \mathrm{~nm}, \pm 0.3 \mathrm{~g} \cdot \mathrm{cm}^{-3}$ and $\pm 0.2 \mathrm{~nm}$, respectively, depending on the model and the system. As such, XRR has been selected in this thesis for in situ monitoring thermal oxidation of $\mathrm{Ru}$ thin films (Chapters 3 and 4), allowing later also, in the case of Chapter 4, to accurately extract the diffusion rates and activation energies for oxygen diffusion through a few nanometer thick ruthenium oxide layers.

It should be noted here that XRR presents also several drawbacks. One of the most important drawbacks is that it does not provide chemical composition of the sample. It also cannot distinguish between layers with relatively small optical contrast. Such that 
the ordering and the composition of the sample layers should be provided in advance. This has been done in this thesis by pre-knowledge of the deposited layered structure and/or by performing chemical compositional analysis of the layers via techniques such as XPS or AES. In addition, specular XRR cannot distinguish between interface roughness and interface interdiffusion, with can be achieved by, for instance, other offspecular reflectivity measurements. ${ }^{288}$ However, this distinction is out of the scope of this thesis. It should be noted here that XRR analysis does not provide us with an absolute and unique description of our layered structures, but allows us an accurate determination of the relative changes in the structure with $\AA$ and, even in some cases, sub- $\AA$ precision.

\subsubsection{XRR principles}

(Specular) XRR normally uses monochromatic X-rays produced from a source to irradiate a sample with certain angles of incidence $\theta$ (usually grazing angles in the range between 0 and $10^{\circ}$ ) with respect to the sample surface, and reflected X-rays are recorded by the detector with the same angle respect to the sample surface, leading to a typically known Bragg Brentano (or $\theta-2 \theta$ ) configuration (see scheme in Fig. 2.7 (a)). The incident $\mathrm{X}$-rays penetrate deep into the sample by refraction, allowing a large probing depth. However, it depends on the radiation energy, the material and the grazing angle $\theta$. For instance, if we irradiate a single Ru layer with a mass density of $12.2 \mathrm{~g} \cdot \mathrm{cm}^{-3},{ }^{38} \mathrm{using}$ an energy of $8051 \mathrm{eV}(\sim 0.154 \mathrm{~nm}$ radiation) at different grazing angles $\theta$ between 1 and $10^{\circ}$, values between $\sim 0.08$ and $0.83 \mu \mathrm{m}$ can be calculated for the respective X-ray attenuation lengths. ${ }^{287}$ It should be noted that the attenuation length of any radiation is defined as the depth along the surface normal where the intensity of this radiation falls to $1 /$ e of its value at the surface. ${ }^{289}$

As any interaction of light with matter, reflection may occur whenever there is a change of the refractive index motivated by the change of medium where the light is travelling. In general, a certain fraction of light is reflected from the interface, and the remainder is refracted into the medium. This reflected fraction of light (the so-called Fresnel reflection) can be obtained by Fresnel equations, where among the different variables, it depends on the incident angle $\theta \cdot{ }^{290}$ In the case of X-rays, the refractive index of a material is close to 1 , with small values of $\delta$. Therefore, X-rays undergo total external reflection when incident on a flat material sample at grazing angles $\theta$ smaller than the critical angle $\theta_{c}{ }^{291}$ The critical angle $\theta_{c}$ can be estimated by:

$$
\theta_{c} \approx \sqrt{2 \delta}
$$

For instance, for $\mathrm{Ru}$ single layer a $\theta_{c}$ value of $\sim 0.47^{\circ}$ can be estimated by Eq. (2.6), when considering a calculated $\delta$ value of $\sim 3.4 \cdot 10^{-5}$ using the previous mass density and X-ray energy radiation. ${ }^{287}$ For incident angles $\theta>\theta_{c}$, the X-rays penetrate into the material by refraction, and the reflected $\mathrm{X}$-rays decrease in a ratio proportional to $\theta^{4}$. (See decrease 
of the reflectivity signal at $2 \theta>\sim 0.9^{\circ}$ in the spectrum of Fig. 2.7 (b) for a Ru single layer on $\mathrm{SiO}_{2}$ onto a $\mathrm{Si}$ substrate).

When considering a layered sample, the reflected X-rays from the surface and the different interfaces, interact with each other via constructive or destructive interference as a consequence of the angular-dependent phase shift, ${ }^{292}$ leading to the formation of oscillations (the so-called Kiessig fringes), ${ }^{293}$ as the ones shown in the XRR spectrum in Fig. 2.7 (b). In this spectrum, the Kiessig fringes originate mainly from the interference of the reflected X-rays from the vacuum/ $\mathrm{Ru}$ interface and the $\mathrm{Ru} / \mathrm{SiO}_{2}$ interface due to the similar optical contrast between the $\mathrm{SiO}_{2}$ layer and the $\mathrm{Si}$ wafer.

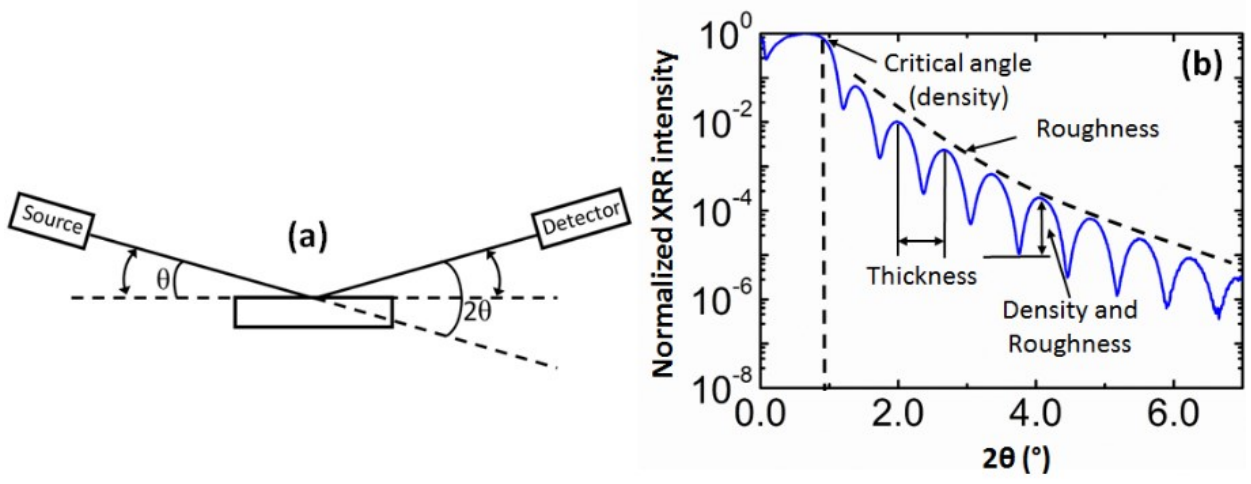

Fig. 2.7. (a) Scheme of XRR measurement geometry. (b) Example of XRR spectrum for an as deposited $\sim 12 \mathrm{~nm}$-thick Ru layer on $\sim 16 \mathrm{~nm}$-thick $\mathrm{SiO}_{2}$ layer on $\mathrm{Si}$ wafer (example of an as deposited sample of Chapters 3 and 4). The critical angle and the information (density, thickness and roughness) extracted from the XRR spectrum are also pointed out in (b).

In general, the $\theta-2 \theta$ configuration is sensitive to the electron density profile perpendicular to the surface. Thus, each X-ray reflectivity spectrum gives an in-depth electron density profile, providing information about the sample layers (thicknesses, densities and intermixing/roughness's). There are several features in the XRR spectrum associated to this information about the sample layers. Following, these features are discussed and presented by means of the previous example spectrum in Fig. 2.7 (b). It should be noted that in this spectrum most of the information is coming from the $\mathrm{Ru}$ layer due to the low optical contrast of the $\mathrm{SiO}_{2} / \mathrm{Si}$ interface, as already mentioned. Thus, it can be considered as an example of a simple single layer with a high optical contrast on a substrate:

Thickness. The period of the Kiessig fringes depends mainly on the $\mathrm{Ru}$ film thickness. The thicker the film, the shorter the period.

Density. The critical angle and the amplitude of the Kiessig fringes depend mainly on the Ru film density. As calculated before for a bulk Ru layer, the critical angle should 
be $\sim 0.9^{\circ}$, as indicated in Fig. 2.7 (b). The amplitude of the fringes depends on the difference between the densities of the $\mathrm{Ru}$ film and its substrate (considering substrate as: $\mathrm{SiO}_{2}+\mathrm{Si}$ ). The larger the density difference, the higher the amplitude of the fringes.

Surface or interface roughness/intermixing. The decay of the reflected intensity at higher angles depends mainly on the $\mathrm{Ru}$ surface roughness (not considering the influence of $\sim 1 \mathrm{ML}^{211}$ oxide on its top). The larger the surface roughness, the faster the reflected intensity decays. The amplitude of the Kiessig fringes decreases mainly with increasing the $\mathrm{Ru} / \mathrm{SiO}_{2}$ interface roughness. The larger the interface roughness, the lower the amplitude of the fringes.

The process of obtaining this information (film thicknesses, densities and roughness's/intermixing) from the XRR spectrum is not that straightforward for up to 4 layer samples presented in this thesis, since there are various reflected waves from different interfaces, interfering with each other, which require a complex model described by Fresnel-like equations and a solving procedure. As such, in this thesis the information from the XRR spectrum is extracted using GenX software. ${ }^{294}$ A layered model of the sample structure is introduced into GenX that consists of the pre-known sample layers. Then, this software simulates a reflectivity spectrum of this layered structure model, and the thickness, density, and roughness of each of the layers in the model are varied using a differential evolution algorithm in order to minimize the differences between simulated and experimental XRR data. Finally, layer thicknesses and layer densities which are the ones of the interest in this thesis, are determined with accuracies of $\pm 0.1 \mathrm{~nm}$ and $\pm 0.3 \mathrm{~g} \cdot \mathrm{cm}^{-3}$, respectively. It should be noted that this program utilizes a Parratt algorithm for simulating X-ray reflectivity, ${ }^{295}$ the roughness is described according to the Névot and Croce model, ${ }^{296}$ and the optical constants used are obtained from CXRO. ${ }^{287}$ A more detailed information about this program can be found in ref. ${ }^{294}$.

\subsubsection{XRR setup}

The setup used in this thesis (all Chapters) for XRR measurements consists of a PANalytical Empyrean X-ray diffractometer $(\mathrm{Cu}-\mathrm{K} \alpha$ radiation, $\lambda=0.154 \mathrm{~nm})$, equipped with a graded $\mathrm{W} / \mathrm{Si}$ multilayer parallel beam mirror that converts the divergent X-ray beam from the X-ray tube to an intense "monochromatic" quasi-parallel beam. It should be noted that although the resulting X-ray beam after the parallel beam mirror is considered to be "monochromatic", it reflects $65 \%$ of the incident radiation into $\mathrm{Cu}-\mathrm{K} \alpha_{1}$ and $\mathrm{Cu}-\mathrm{K} \alpha_{2}$ lines in an aspect ratio of $2: 1$. However, the $\mathrm{Cu}-\mathrm{K} \beta$ is suppressed to below $0.5 \%$. To reduce the height of the X-ray beam, and hence control the amount of sample irradiated, a divergence slit of $1 / 32^{\circ}$ is placed before the mirror. In addition, an antiscatter slit of $0.8 \mathrm{~mm}$ (diameter) is placed before the detector to limit the acceptance angle of the detector and reduce the scattered background. An automated beam attenuator 
is mounted after the mirror to avoid damage of the detector during illumination. This setup incorporates the DHS 1100 sample stage (described in Sec. 2.2.1) for annealing experiments, where the sample is covered by an X-ray transparent graphite dome upon heating. This complete configuration provides measurements with higher peak intensity when compared to using a monochromator, allowing shorter measurement times $(\sim 10$ min) for in situ measurements during annealing (Chapters 3 and 4). Although, the resolution is usually lower than for a monochromator, it gives enough resolution for measurements which go from incident angles $\theta$ of $0^{\circ}$ up to $4^{\circ}$ in all Chapters. An image of this XRR setup is shown in Fig. 2.8 with the sample uncovered by the dome.

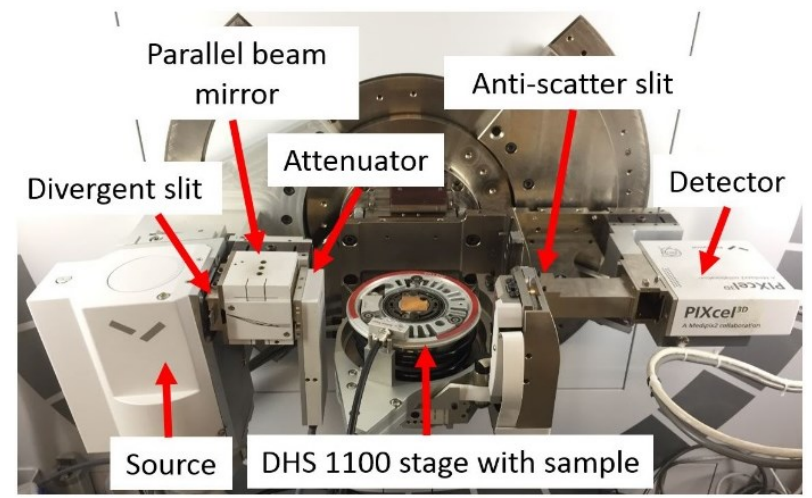

Fig. 2.8. Image of the $\mathrm{Cu}-\mathrm{K} \alpha$ diffractometer for XRR measurements, equipped with a DHS 1100 heating stage and a sample uncovered by the dome.

\subsubsection{X-ray diffraction}

\subsubsection{Motivation for its use}

(Wide angle) X-ray diffraction (XRD), or also called (thin-film) XRD, is commonly used to determine the crystalline structure of layered thin film samples. It gives information such as the crystal phase (or orientation), the crystal size, and the residual stress in the film. ${ }^{297}$ In this thesis (Chapters 3 and 4), we use this technique to extract information only related to the first two features of our thin layered structures: the crystal phase and the crystal size. First, it allows us to distinguish between amorphous and crystalline structures. However, there are cases when the crystalline structure exhibits crystals with sizes $<\sim 1 \mathrm{~nm}$, where this distinction is almost experimentally inviable. We are usually speaking about "X-ray amorphous phase" when we are unable to detect any diffraction peaks, and the whole diffraction spectrum consists only of a few diffuse maxima not easily indexed to any crystal phase, as observed for the $2 \mathrm{~nm}$-thick low-O $\mathrm{ZrO}_{2}$ on a-Si samples (Chapter 6). In the case that there are clear diffraction patterns from larger (detected) crystals, it allows us to reveal the crystalline structure of our thin films which can be composed by crystals either randomly oriented or with a certain 
preferred orientation (texture). ${ }^{297}$ Second, we use the crystal size information from certain orientations, correlating it with the X-ray reflectivity thickness (Chapters 3 and 4), or the "effective" thickness and the surface morphology from AFM (Chapter 3 ) in order to understand the growth of crystalline $\mathrm{RuO}_{2}$ films and the consumption of crystalline $\mathrm{Ru}$ films during thermal oxidation. Such that we study Ru thermal oxidation by in situ monitoring the crystal size of both films.

\subsubsection{XRD principles}

(Thin-film) XRD uses similar principles as (specular) XRR (see Sec. 2.3.5.2). However, for (specular) XRR, the reflected X-rays which provoke constructive and destructive interference based on Bragg's condition are coming from the surface and the different interfaces between layers where there is a change of the refractive index. Whereas for (thin-film) XRD, the X-rays are reflected (or diffracted) from the different crystal planes of atoms of the crystalline layers, resulting in diffraction peaks when Bragg's condition is achieved (see XRD spectrum in Fig. 2.9 (b)).

The measurement configuration for (thin-film) XRD is usually asymmetric, in contrast to the Bragg Brentano (symmetric) configuration for (specular) XRR. Such that for (thin-film) XRD, the incident beam from the source makes a fixed and usually small angle $\alpha\left(<10^{\circ}\right)$ with the sample surface and the detector moves along a circle centered at the sample position. This means that the reflection (or diffraction) comes from different crystal planes which are inclined at different angles with respect to the sample surface, not from parallel interfaces as in the case of XRR. ${ }^{297}$ However, the diffraction angle $2 \theta$ is defined with respect to the incident beam direction as the reflection angle in XRR (see scheme in Fig. 2.9 (a)). For the measurements in this thesis (Chapter 3 and 4) the incident angle $\alpha$ is chosen to be $1^{\circ}$ in order to maximize the sample illumination. The diffraction patterns are recorded by the detector from diffraction angles $2 \theta$ between 20 to $150^{\circ}$ (the maximum $2 \theta$ angle allowed in our setup). This implies Bragg diffraction peaks from atomic crystal planes inclined at angles between 9 to $74^{\circ}$ with respect to the sample surface. It should be noted that the measuring samples are rotated by $20^{\circ}$ in plane with respect to the (100) axis to suppress the possible diffraction peaks coming from the $\operatorname{Si}(100)$ substrate.

For each crystalline structure of a certain material there are different orientations with a certain characteristic inter-planar spacing ( $d$-spacing) that can be obtained from a powder diffraction database such as NIMS. ${ }^{298}$ By comparing the "theoretical" $d$-spacing from this database with the experimental $d$-spacing for each diffraction peak in the XRD spectrum, it is possible to identify their corresponding crystal orientation. Each diffraction angle $2 \theta$ is correlated to the $d$-spacing by Bragg's equation: 


$$
m \lambda=2 d \sin \left(\frac{2 \theta}{2}\right),
$$

where $m$ (an integer) is the order of "reflection", and $\lambda$ is the wavelength of the incident $\mathrm{X}$-rays. For instance, if we consider the first $\mathrm{Ru}$ diffraction peak at $2 \theta$ equal to $\sim 38.3^{\circ}$ from the XRD spectrum in Fig. 2.9 (b), a $d$-spacing of $\sim 2.35 \AA$ can be obtained by Eq. (2.7), using $m=1$ and $\lambda=0.154 \mathrm{~nm}$. This experimental $d$ value can be compared to the "theoretical" $d$ value of $\sim 2.34 \AA,{ }^{299}$ which corresponds to the $\mathrm{Ru}(100)$ orientation, ${ }^{298}$ as pointed out in Fig. 2.9 (b).
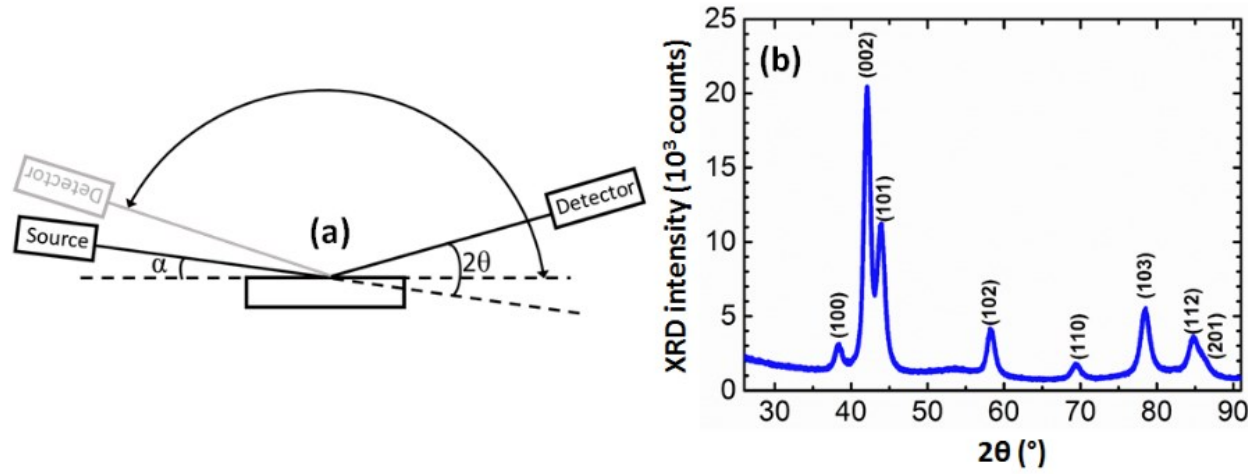

Fig. 2.9. (a) Scheme of (thin-film) XRD measurement geometry. (b) Example of XRD spectrum for an as deposited $\sim 12 \mathrm{~nm}$-thick $\mathrm{Ru}$ layer on $\sim 16 \mathrm{~nm}$-thick $\mathrm{SiO}_{2}$ layer on $\mathrm{Si}$ wafer (example of an as deposited sample of Chapters 3 and 4). The diffraction peaks corresponding to the different orientations from the polycrystalline Ru layer are indicated in (b).

There are several methods to determine the crystal size from XRD. In this thesis, we use the Scherrer equation to determine the crystal size $L$, as:

$$
L=\frac{K \lambda}{B(2 \theta) \cdot \cos \theta},{ }^{300,301}
$$

where $B(2 \theta)$ is the full width at half-maximum (FWHM) of the peak at a diffraction angle $2 \theta, K$ is the Scherrer constant, and $\lambda$ is the wavelength. The exact value of $K$ depends on the actual shape of the crystal and on the Miller indices $h k l$. However, this constant does not display a wide variation with the crystal shape or with $h k l$ for the FWHM approach, and a value of 0.90 can be taken as a first approximation. ${ }^{300}$ Still, for $\mathrm{Ru}$ and $\mathrm{RuO}_{2}$ crystals analyzed in this thesis, respective average $K$ values of 0.88 and 0.94 are considered according to ref. ${ }^{302}$. It should be noted that the crystal size $L$ from Eq. (2.8) is an affective dimension which corresponds to the volume average over the layer of interest of the crystal dimension normal to the diffracting planes. In addition, the detected width of the diffraction peaks $B_{o b s}$ includes two main contributions to its broadening: the crystal size broadening $B(2 \theta)$, and the instrumental broadening $b$. Thus, $B(2 \theta)$ introduced

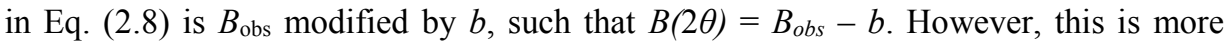
complex because the diffraction peaks are usually a convolution of Lorentzian and 
Gaussian distributions, which need to take into account together with the different radiation lines normally reflected from a parallel beam mirror. WinPLOTR software has been used for fitting the diffraction peaks in this thesis, using a Pseudo-Voigt function. ${ }^{303}$ However, for an accurate determination of the crystallite size, the instrumental broadening $b$ should be small compared to $B_{o b s}$. The instrumental broadening for our instrument and configuration is $\sim 0.43^{\circ}$. Roughly, crystal sizes $>22 \mathrm{~nm}$ will have comparable $B_{o b s}$ as this instrumental broadening, leading to significant inaccuracies. For crystal sizes corrected by this instrumental broadening, the estimated accuracies are between $\sim 10$ to $25 \%$. Still reasonable for the purpose of the crystal size determination presented in this thesis (Chapters 3 and 4).

\subsubsection{XRD setup}

The setup used in this thesis for (thin-film) XRD measurements (Chapters 3 and 4) is the same as the one used for XRR measurements (see Sec. 2.3.5.3). However, instead of using only an anti-scatter slit before the detector, a parallel plate collimator is placed in this location. It limits the $2 \theta$ divergence/acceptance angle of the detector to $0.27^{\circ}$, providing the main part of the instrumental broadening of our setup. In addition, a divergent slit of $1 / 8^{\circ}$ is used before the parallel beam mirror in order to maximize the irradiated area with enough resolution.

\subsubsection{Atomic force microscopy}

\subsubsection{Motivation for its use}

A combined 2D (thin film) and 3D (island) oxide growth has been observed for thermal oxidation of monocrystalline $\mathrm{Ru} .{ }^{223,224}$ However, for polycrystalline $\mathrm{Ru}$, no 3D oxide growth has been observed, ${ }^{265,266}$ with only increased roughening being detected. ${ }^{267}$ In this thesis, we want to have a tool that allows us to recognize such (possible) 3D features growing in our relevant polycrystalline $\mathrm{Ru}$ films during thermal oxidation. Therefore, we have used atomic force microscopy (AFM) in this thesis for this purpose (Chapter 3). This technique is a high-resolution type of scanning probe microscopy which allows to study the surface morphology (3D topography) of such samples with sub-nanometer height resolution and a lateral resolution of several tens of nanometers. In addition, AFM provides information about the roughness development during growth, and it is also used for this purpose during growth of $\mathrm{Ru}$ and $\mathrm{ZrO}_{2}$ films on different substrate layers (Chapters 5 and 6).

Although AFM exhibits advantages compared to other scanning microscopies such as SEM, being able to create 3D surface profiles instead of only 2D sample images, it also presents several disadvantages. One of the main drawbacks is the formation of image artifacts due to the geometrics (or the shape) of the scanning probe, such that certain 
surface features cannot be properly resolved. For instance, the surface rectangular-like $\mathrm{RuO}_{2}$ nano-columns are detected by AFM as semi-spherical elongated agglomerates, such that their "real" shape cannot be resolved, as presented in this thesis (Chapter 3).

\subsubsection{AFM principles}

AFM uses a microscale cantilever equipped with a sharp tip to probe the sample surface. ${ }^{304}$ This tip exhibits a radius of curvature usually between $\sim 1$ to $20 \mathrm{~nm}$, depending on the required lateral resolution. For instance, the tips used for Chapters 5 and 6 , are $\mu$ masch Hi'Res-C14 with a tip radius of $1 \mathrm{~nm}$, leading to a lateral resolution theoretically below $1 \mathrm{~nm}$. When the tip is close to the sample surface, there appear forces between the tip and the sample, which lead to the deflection of the cantilever, following Hooke's law. ${ }^{305}$ This deflection can be monitored with different sensing systems such an optical laser. AFM can be operated in several modes, depending on the application. In this thesis the tapping mode is used, which usually prevents fast wear of the tip. This is due to the fact that the oscillating operation of the tip in this mode, prevents it from sticking to the surface, while still penetrating the liquid meniscus surface layer that normally develops in ambient conditions. More information about this mode can be found in ref. ${ }^{304}$.

\subsubsection{AFM setup}

The AFM setup used in this thesis for imaging (Chapters 3, 5 and 6) is a BRUKER Dimension Edge atomic force microscope, and the image analysis (roughness, line profiles) is performed by WSxM software. ${ }^{306}$

\subsubsection{Transmission electron microscopy}

\subsubsection{Motivation for its use}

We want to have a technique that allows us to resolve structural details in atomic level, including crystal planes, grain boundaries, or other small features. In particular, we need a tool that allows us to resolve the shape of 3D oxide nano-columns growing on the $\mathrm{Ru}$ surface during thermal oxidation (Chapter 3). Within the limitations of AFM, these features are detected by this technique, exhibiting semi-spherical elongated shapes. However, transmission electron microscopy (TEM) provides us with the "real" structure of these features, exhibiting a rectangular-like shape rather than a semi-spherical shape. In addition, TEM also allows the identification of the crystalline structure of these columns and the underneath layers, providing a complete understanding of our thermal oxidized $\mathrm{Ru}$ films in Chapter 3. 


\subsubsection{TEM principles}

TEM uses a focused electron beam with an energy between 40 to $400 \mathrm{keV}$ to penetrate through an electron transparent sample. The sample structure and composition affects its electron absorption, leading to a 2D image when the transmitted electrons hit a fluorescent screen or CCD camera, after a large $\left(\sim 10^{4}-10^{6}\right)$ magnification by several lenses. Due to electron absorption, sample regions with high-Z elements appear darker on TEM images, while sample regions with low-Z elements appear brighter.

TEM provides images with high-resolution down to sub-nanometer or nanometer scales, which is typically higher resolution than SEM. But this strongly depends on the equipment, magnification and specimen. For instance, an aberration-corrected TEM can achieve a resolution below $0.5 \AA$ at magnifications above 50 million times, by using high electron transparent samples. ${ }^{307}$ Apart from imaging, it usually provides elemental identification by spectral analysis of refracted and scattered electrons in electron energy loss spectroscopy (EELS) and energy dispersive X-ray analysis (EDX). Although these two techniques are also performed in our samples imaged by TEM (Chapter 3), their results are not presented in this thesis.

Compared to SEM, all samples cannot be directly analyzed by TEM, since their thicknesses need to be typically between $\sim 100$ to $300 \mathrm{~nm}$ to ensure electrons to pass through for the measurement. ${ }^{308}$ Planar samples are normally fabricated by depositing thin structures on a special TEM grid designed for TEM analysis. For layered samples such as the ones presented in this thesis (Chapter 3), a sample cross-section is normally preferred for TEM analysis. This allows to visualize the layers and the interfaces between layers. However, it requires an extensive sample preparation to produce a thin enough electron transparent sample cross-section. A detailed information of sample preparation can be found in ref. ${ }^{309}$.

Apart from the required extensive sample preparation, there are other drawbacks from cross-sectional TEM analysis. First, during this preparation the sample can be altered. For instance, a large part of $\mathrm{RuO}_{2}$ nano-columns are detached from the sample surface during TEM preparation, as presented in Chapter 3. In addition, the energetic electron beam can damage the sample during analysis.

\subsubsection{TEM setup}

The (cross-sectional) high-resolution TEM (HR-TEM) setup used in this thesis (Chapter 3) is a Philips CM300ST-FEG (S)TEM instrument, installed in the MESA+ Nanolab facilities at the University of Twente. At $300 \mathrm{kV}$, it allows a point and line resolution for imaging of 0.2 and $0.14 \mathrm{~nm}$, respectively. The image analysis is carried out by DigitalMicrograph software. ${ }^{310}$ 


\title{
3. Surface and sub-surface thermal oxidation of thin ruthenium films
}

\begin{abstract}
A mixed 2D (film) and 3D (nano-column) growth of ruthenium oxide has been experimentally observed for thermally oxidized polycrystalline ruthenium thin films. Furthermore, in situ X-ray reflectivity upon annealing allowed the detection of 2D film growth as two separate layers consisting of low density and high density oxides. Nanocolumns grow at the surface of the low density oxide layer, with the growth rate being limited by diffusion of ruthenium through the formed oxide film. Simultaneously with the growth of the columns, sub-surface high density oxide continues to grow limited by diffusion of oxygen or ruthenium through the oxide film.
\end{abstract}




\subsection{Introduction}

Ruthenium thin films and its oxidized compounds raised great interest in many applications in the recent years. ${ }^{14} \mathrm{Ru}$ has turned out to be the most active catalyst in the synthesis of ammonia, ${ }^{1,2}$ while $\mathrm{RuO}_{2}$ has shown to be an excellent oxidation catalyst in heterogeneous catalysis ${ }^{311}$ and electrocatalysis. ${ }^{312}$ Other applications for $\mathrm{Ru}$ thin films are as bottom electrode in VLSI capacitors based on high dielectric materials, ${ }^{5}$ or as capping layer for optics designed for extreme ultraviolet lithography (EUVL) ${ }^{7,49,132}$ due to its low-oxidation properties. ${ }^{11}$ Oxidation of monocrystalline $\mathrm{Ru}$ was extensively investigated during the last decades. ${ }^{230,262,263,264,313,314,315,316}$ Recently, oxide thin films and $3 \mathrm{D}$ clusters were observed during thermal oxidation of single crystalline $\mathrm{Ru} .{ }^{223,224}$ For polycrystalline Ru no 3D clusters have been seen, with only thin film oxide growth being observed. . $^{265,266,267}$

In this chapter, we report on the simultaneous 2D (thin film) and 3D (nano-column) growth of ruthenium oxide experimentally observed for thermally oxidized polycrystalline $\mathrm{Ru}$ thin films. Furthermore, it is also found that the thin film oxide does not grow as a single layer but a combination of two layers on top of each other.

To determine parameters of nanometer range thin films and dynamics of their growth accurately, an in situ technique needs to be applied. Previously in situ spectroscopic ellipsometry (SE) was used to study growth of thin $\mathrm{RuO}_{2}$ films during thermal oxidation of Ru. ${ }^{283,284}$ However, it should be noted that extracting information from SE involves reconstruction of both optical constants and layered structure, a process which is often non-uniquely determined. We used in situ hard X-ray reflectivity measurements for monitoring thermal oxidation of Ru thin films. The changes of the indepth electron density distribution were accurately determined from the changes of the reflectivity patterns during the thin film growth. This provided us with information about densities, thicknesses and intermixing/roughnesses of the formed $\mathrm{RuO}_{\mathrm{x}}$ and remaining $\mathrm{Ru}$ layers during the oxidation process. Combining this with Auger electron spectroscopy, angular-resolved X-ray photoelectron spectroscopy, atomic force microscopy, X-ray diffraction and high-resolution transmission electron microscopy, we present a detailed description of surface and sub-surface oxidation of the ruthenium thin films and propose a model for concurrent 2D and 3D ruthenium oxide growth.

\subsection{Experimental}

Ten nanometer thick Ru films were deposited onto natively oxidized super-polished Si substrates using DC magnetron sputtering ( $1 \times 10^{-3}$ mbar Ar) in a UHV setup with base pressure $<1 \times 10^{-8}$ mbar. Since $\mathrm{Ru}$ and $\mathrm{Si}$ intermix upon annealing, ${ }^{145,317}$ an additional $15 \mathrm{~nm} \mathrm{SiO} 2$ diffusion barrier was deposited on top of the Si substrate before 
$\mathrm{Ru}$ deposition. $\mathrm{SiO}_{2}$ was chosen for this purpose due to its low enthalpy of formation of $-910.7 \mathrm{~kJ} \cdot \mathrm{mol}^{-1},{ }^{38}$ compared to -32.4 and $-26.0 \mathrm{~kJ} \cdot \mathrm{mol}^{-1}$ for $\mathrm{RuSi}$ and $\mathrm{Ru}_{2} \mathrm{Si}_{3}$, respectively. ${ }^{318}$ Layer thicknesses were monitored with quartz mass balances during deposition and used as initial fit parameters for X-ray reflectivity analysis.

The deposited $\mathrm{Ru}$ films were thermally oxidized at temperatures between 150 to $600^{\circ} \mathrm{C}$ for different amount of time. X-ray reflectivity (XRR) measurements were conducted in situ during annealing using a PANalytical Empyrean X-Ray diffractometer $\left(\mathrm{Cu}-\mathrm{K} \alpha\right.$ radiation, $0.154 \mathrm{~nm}$ ), equipped with an Anton Paar thermal stage. ${ }^{273}$ Before annealing, the alignment of the sample position with respect to the impinging $\mathrm{X}$-ray beam was performed and a reference XRR reflectivity curve was recorded. After heating the sample to an elevated temperature, the sample position with respect to the beam was realigned to correct for thermal expansion and possible misalignment, and subsequent XRR scans were recorded during annealing.

X-ray reflectivity curves were analyzed using the GenX software. ${ }^{294}$ A layered model of the structure was composed that consists of the $\mathrm{Si}$ substrate, a $\mathrm{SiO}_{2}$ layer, a $\mathrm{Ru}$ layer and an oxide layer. Hard X-rays used will fully penetrate the films. However, the reflectivity is dominated by the interfaces with high optical contrast such as vacuum/ $\mathrm{RuO}_{\mathrm{x}}, \mathrm{RuO}_{\mathrm{x}} / \mathrm{Ru}$, and $\mathrm{Ru} / \mathrm{SiO}_{2}$. The $\mathrm{SiO}_{2} / \mathrm{Si}$ interface has very low optical contrast and does not affect the analysis. The GenX program varied thickness, roughness and density of each of the layers in order to minimize the differences between model simulations and experimental data. Layer thicknesses and layer densities were determined with accuracies of $\pm 0.1 \mathrm{~nm}$ and $\pm 0.3 \mathrm{~g} \cdot \mathrm{cm}^{-3}$, respectively.

\subsection{Results and discussion}

Fig. 3.1 presents an example of the measured and simulated XRR data for (a) an as deposited and (b) annealed sample at $400^{\circ} \mathrm{C}$ for 20 minutes.

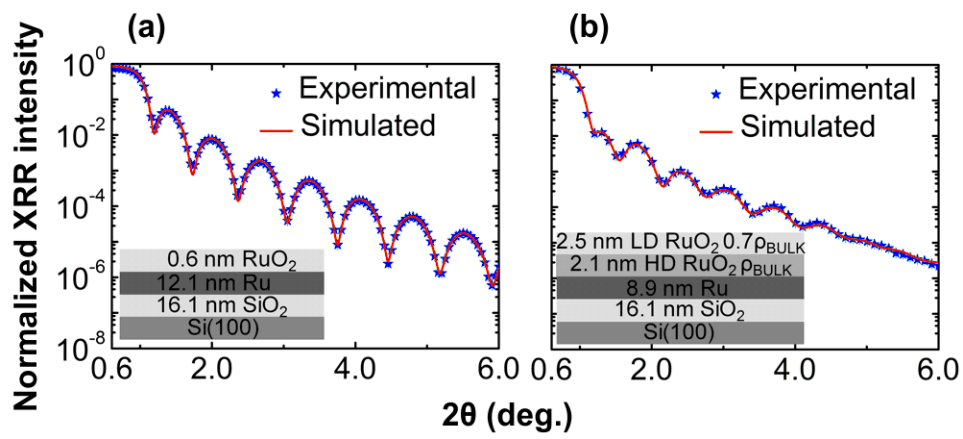

Fig. 3.1. Experimental (stars) and simulated (line) specular X-ray reflectivity (XRR) data for (a) an as deposited sample and (b) a sample annealed at $400^{\circ} \mathrm{C}$ for 20 minutes. Left-bottom (a, b) inset shows the layered model used for each simulation. 
According to XRR of the as-deposited sample (see Fig. 3.1(a)), there exists a $\mathrm{RuO}_{2}$ layer of $0.6 \pm 0.1 \mathrm{~nm}$ on top of $\mathrm{Ru}$. The presence of a monolayer (ML) of oxide on the asdeposited sample was confirmed by XPS measurements. As a low-oxidation material, ${ }^{11}$ $\mathrm{Ru}$ is known to remain metallic, and only $1 \mathrm{ML}$ of oxide is typically chemisorbed at room temperature. ${ }^{205,209,211,311,319}$ With increasing temperature, the Ru layer oxidizes further and XRR suggests a gradual growth of a low density oxide layer of approximately $6.0 \pm 0.3 \mathrm{~g} \cdot \mathrm{cm}^{-3}$. Above $325^{\circ} \mathrm{C}$, XRR data cannot be modelled using a single low density oxide layer and there is a need for a 2-layer oxide model to describe the thin film oxide growth (see Fig. 3.1(b)). In this model, a low density (LD) $\mathrm{RuO}_{2}$ layer is formed on top of a high density (HD) $\mathrm{RuO}_{2}$ layer, with $\mathrm{LD}$ and $\mathrm{HD}$ oxide densities being $5.3 \pm 0.3$ and $6.8 \pm 0.3 \mathrm{~g} \cdot \mathrm{cm}^{-3}$, respectively, which corresponds to $70 \%$ and $100 \%$ of the bulk $\mathrm{RuO}_{2}$ density.

To confirm the 2-layer oxide structure observed by XRR, angular-resolved photoelectron spectroscopy (AR-XPS) measurements were performed for the samples annealed at temperatures $\geq 325^{\circ} \mathrm{C}$. Fig. 3.2 presents the Ru-3d XPS spectrum recorded at $\sim 26^{\circ}$ off-normal detection (a), and the atomic percentage from the $\mathrm{Ru}-3 \mathrm{~d}_{5 / 2}$ peaks, corresponding to the different $\mathrm{Ru}$ species $\left(\mathrm{Ru}, \mathrm{RuO}_{2}\right.$ and $\mathrm{RuO}_{\mathrm{x}}$, where $2<\mathrm{x} \leq 3$ ) as a function of the detection angle (b) for a $20 \mathrm{~min}$ annealed sample at $325^{\circ} \mathrm{C}$.

(a)

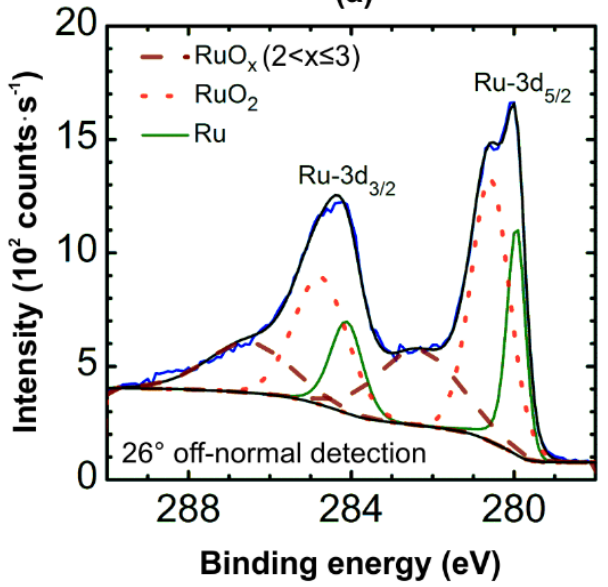

(b)

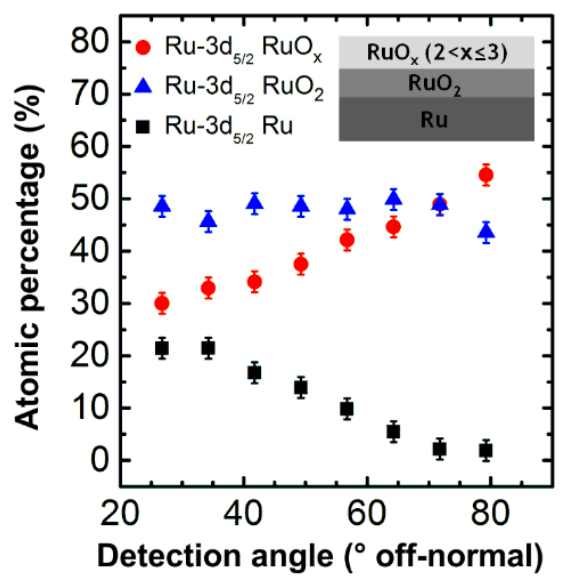

Fig. 3.2. Ru-3d XPS spectrum recorded at $\sim 26^{\circ}$ off-normal detection (a), and atomic percentage from the $\mathrm{Ru}-3 \mathrm{~d}_{5 / 2}$ peaks which correspond to $\mathrm{Ru}$ (squares), $\mathrm{RuO}_{2}$ (triangles) and $\mathrm{RuO}_{\mathrm{x}}$ (circles) species as a function of detection angle (b) for a $20 \mathrm{~min}$ annealed sample at $325^{\circ} \mathrm{C}$. Ru-3 $\mathrm{d}_{3 / 2}$ (left) and $\mathrm{Ru}-3 \mathrm{~d}_{5 / 2}$ (right) peaks which correspond to elemental $\mathrm{Ru}$ (green solid lines), $\mathrm{RuO}_{2}$ (red dotted lines), and $\mathrm{RuO}_{\mathrm{x}}(2<\mathrm{x} \leq 3)$ (brown dashed lines) are indicated in (a). Inset in (b) shows the layered model suggested from AR-XPS for higher detection angles, corresponding to a probed volume closer to the surface. 
Two $\mathrm{Ru}$ oxide species $\left(\mathrm{RuO}_{2}\right.$ and $\mathrm{RuO}_{\mathrm{x}}$, where $\left.2<\mathrm{x} \leq 3\right)$ are detected by XPS at annealing temperatures $\geq 325^{\circ} \mathrm{C}$ (Fig. 3.2 (a), dotted and dashed lines, respectively). Apart from the $\mathrm{RuO}_{2}$ peak at $\sim 280.7 \mathrm{eV}$ (Fig. 3.2(a), red dotted line), the peak assigned to $\mathrm{RuO}_{\mathrm{x}}(2<\mathrm{x} \leq 3)$ at $\sim 282.2 \mathrm{eV}$ (Fig. 3.2(a), brown dashed line) has also been observed by other XPS studies on oxidized $\mathrm{Ru}$, and attributed to a $\mathrm{RuO}_{2}(110)-\mathrm{O}_{\text {ot }}$ complex at similar binding energy. ${ }^{320,321}$ In particular, this peak has been associated to $1 \mathrm{f}$-cus- $\mathrm{Ru}$ atoms that are capped by on-top oxygen ( $1 \mathrm{f}$-cus- $\mathrm{Ru}+\mathrm{O}_{\mathrm{ot}}$ ), forming part of the $\mathrm{RuO}_{2}(110)$ $\mathrm{O}_{\text {ot }}$ complex that could be envisioned as a $\mathrm{RuO}_{3}$ surface species, since the $\mathrm{O}_{\mathrm{ot}}$ is not shared with other Ru atoms. ${ }^{320}$ It should be noted that our observed $\mathrm{RuO}_{\mathrm{x}}$ peak should not be confused with a possible peak appearing at $\sim 282.7 \mathrm{eV}$, usually associated to a satellite due to excitation of a $\mathrm{RuO}_{2}$ plasmon, ${ }^{320}$ and hence it might be associated to an oxygen-rich $\mathrm{RuO}_{\mathrm{x}}$ phase. From the evolution of the atomic percentage from the $\mathrm{Ru}, \mathrm{RuO}_{2}$ and $\mathrm{RuO}_{\mathrm{x}}$ peaks measured at different detection angles, we establish a layered model structure of our oxidized $\mathrm{Ru}$ samples (see model in Fig. 3.2(b) for a 20 min annealed sample at $\left.325^{\circ} \mathrm{C}\right)$. As such, AR-XPS confirms the gradation of the oxide film and indicates that the top part of the layer is oxygen rich, suggesting a $\mathrm{RuO}_{\mathrm{x}} / \mathrm{RuO}_{2}(2<\mathrm{x} \leq 3)$ layer structure, which corresponds to the similar 2-layer oxide model structure obtained by XRR.

Fig. 3.3 (left-axis) shows an example of the oxide layer thickness (low density and high density) and $\mathrm{Ru}$ consumption as derived from XRR, as function of annealing time at $400^{\circ} \mathrm{C}$. The ratio of thicknesses between the total $\mathrm{RuO}_{2}$ formed (low density and high density) and Ru lost in time is also plotted in Fig. 3.3 (right-axis).

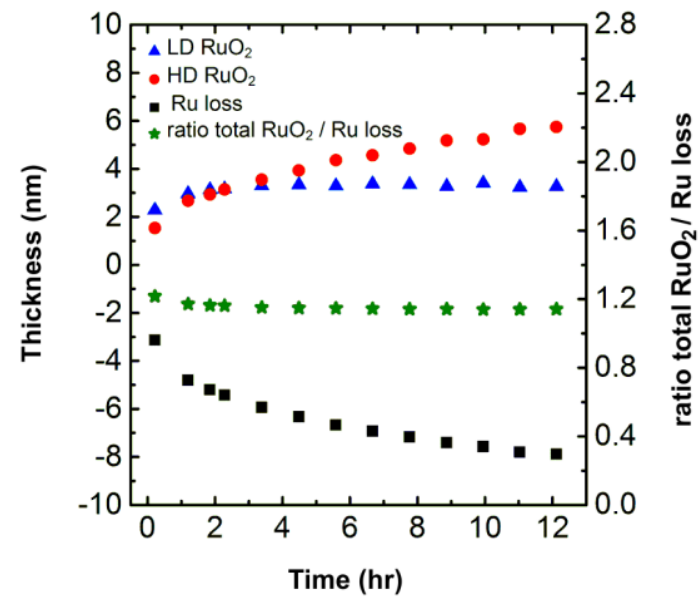

Fig. 3.3. Left-axis: $\mathrm{RuO}_{2}$ thickness and $\mathrm{Ru}$ loss as a function of annealing time for $400^{\circ} \mathrm{C}$. Low density (LD) $\mathrm{RuO}_{2}$ thickness (triangles), high density (HD) $\mathrm{RuO}_{2}$ thickness (circles) and $\mathrm{Ru}$ loss (squares) are plotted. Right-axis: ratio between total $\mathrm{RuO}_{2}$ formed (summed low density and high density) and $\mathrm{Ru}$ loss as a function of annealing time for $400^{\circ} \mathrm{C}$ (stars). The layer thicknesses were determined with the accuracy of $\pm 0.1 \mathrm{~nm}$. 
The low density $\mathrm{RuO}_{2}$ layer rapidly saturates at a thickness of approximately $3 \mathrm{~nm}$, while the high density $\mathrm{RuO}_{2}$ layer continues to grow, as depicted by triangles and circles in Fig. 3.3 (left-axis). The Ru layer thickness decreases over time, consistent with the consumption of $\mathrm{Ru}$ during formation of $\mathrm{RuO}_{2}$, and is plotted as $\mathrm{Ru}$ loss by squares in Fig. 3.3 (left-axis). The ratio of thicknesses between the total $\mathrm{RuO}_{2}$ formed (low density and high density) and $\mathrm{Ru}$ lost in time shows a constant value of $\sim 1.2$, as depicted by stars in Fig. 3.3 (right-axis). Distinctly, based on calculations assuming bulk densities this ratio is expected to be 2.3. This surprising discrepancy has been resolved when studying morphology of the sample surface with AFM, XRD and HR-TEM.

The top surface of the oxidized $\mathrm{Ru}$ surface was studied by atomic force microscopy (AFM) for the samples annealed for 20 minutes at temperatures between 150 and $500^{\circ} \mathrm{C}$. Fig. 3.4 shows the $1 \times 1 \mu \mathrm{m}$ AFM images for as deposited $\mathrm{Ru}(\mathrm{a})$, and 20 minutes annealed samples at $175^{\circ} \mathrm{C}$ (b), $200^{\circ} \mathrm{C}$ (c), $300^{\circ} \mathrm{C}$ (d), $400^{\circ} \mathrm{C}$ (e), and $500^{\circ} \mathrm{C}$ (f).

(a)

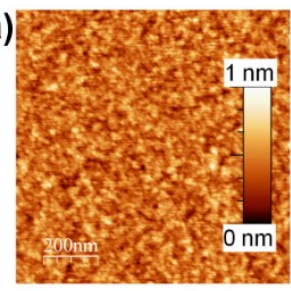

(c)
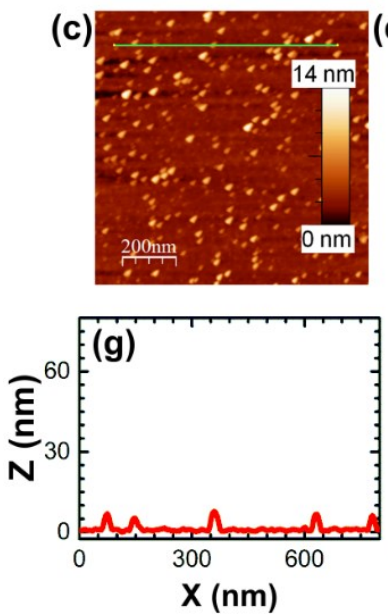
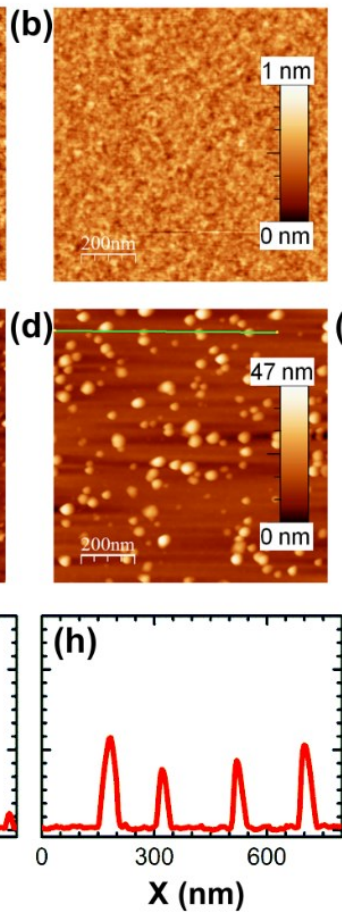
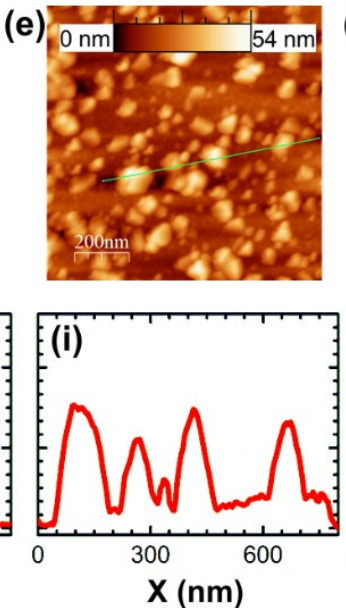
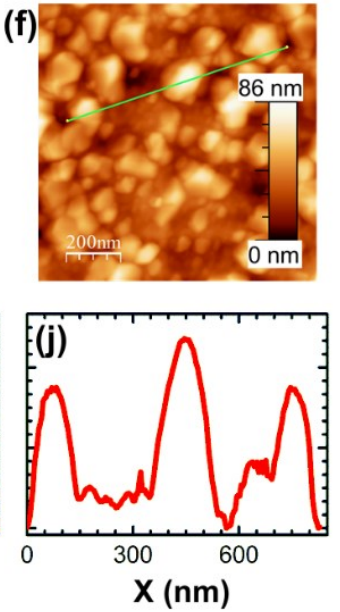

Fig. 3.4. Atomic force microscopy (AFM) images $(1 \times 1 \mu \mathrm{m})$ of the as deposited (a), and 20 minutes annealed samples at $175^{\circ} \mathrm{C}$ (b), $200^{\circ} \mathrm{C}(\mathrm{c}), 300^{\circ} \mathrm{C}(\mathrm{d}), 400^{\circ} \mathrm{C}(\mathrm{e})$, and $500^{\circ} \mathrm{C}(\mathrm{f})$. Line profiles of the 20 minutes annealed samples at $200^{\circ} \mathrm{C}(\mathrm{g}), 300^{\circ} \mathrm{C}(\mathrm{h}), 400^{\circ} \mathrm{C}(\mathrm{i})$, and $500^{\circ} \mathrm{C}$ (j) are represented by the straight lines shown in the AFM images (c), (d), (e), and (f), respectively.

The as deposited and $175^{\circ} \mathrm{C}$ annealed samples present a similar root mean square (RMS) value of $\sim 0.25 \mathrm{~nm}$ (see Figs. 3.4(a) and 3.4(b)). The surface morphology start to 
change significantly when the samples are annealed at $200^{\circ} \mathrm{C} .3 \mathrm{D}$ columns appear at the surface and grow in size with increasing oxidation temperature (Figs. 3.4(c), 3.4(d), 3.4(e), and 3.4(f)). Line profiles along the lines indicated in Figs. 3.4(c), 3.4(d), 3.4(e), and 3.4(f) are presented in Figs. 3.4(g), 3.4(h), 3.4(i), and 3.4(j) and show the increasing evolution of the columns with average heights of $\sim 7 \mathrm{~nm}, \sim 30 \mathrm{~nm}, \sim 37 \mathrm{~nm}$, and $\sim 53 \mathrm{~nm}$ at $200^{\circ} \mathrm{C}, 300^{\circ} \mathrm{C}, 400^{\circ} \mathrm{C}$, and $500^{\circ} \mathrm{C}$, respectively. To determine the elemental composition of the oxidized Ru surface, Auger electron spectroscopy (AES) was carried out on both columns and on the areas between the columns on the surface of the annealed samples. It should be noted that due to the relatively low $(\sim 1 \mu \mathrm{m})$ lateral resolution of our AES setup, only samples with columns with lateral sizes higher than this resolution limit could be resolved. This restricted the analysis to the samples annealed at temperatures $>500^{\circ} \mathrm{C}$, where the column lateral size strongly increased up to a few $\mu \mathrm{m}$. As an example, AES spectra of a column and of the area between the columns for a 10 min annealed sample at $600^{\circ} \mathrm{C}$ are plotted by solid and dashed lines in Fig. 3.5(a). The corresponding measurement points are depicted in the $400 \times 400 \mu \mathrm{m}$ SEM image of this sample (Fig. $3.5(\mathrm{~b}))$.

(a)

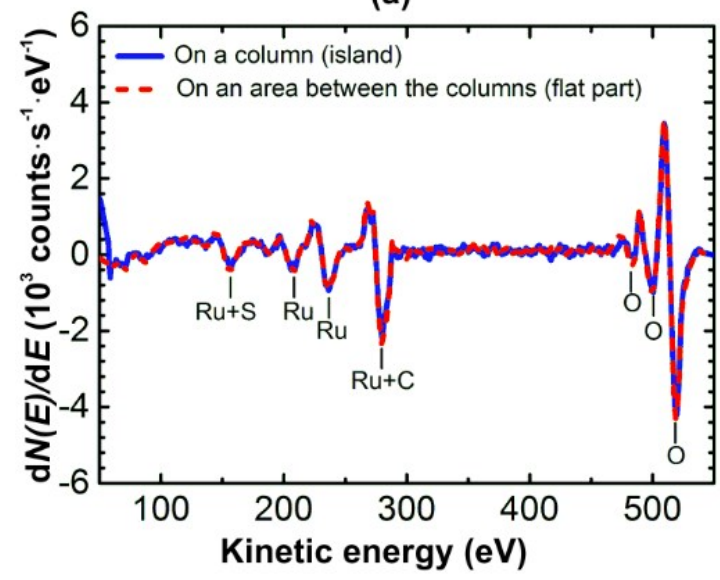

(b)

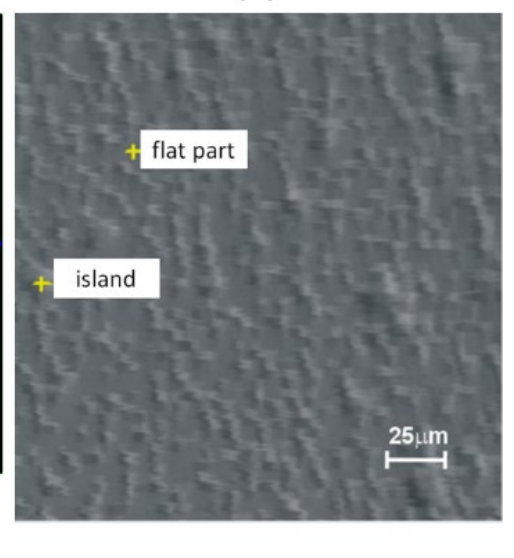

Fig. 3.5. AES spectra of a column (solid line) and of the area between the columns (dashed line) for a 10 min annealed sample at $600^{\circ} \mathrm{C}$ (a). The surface elements are also indicated in (a) for the different AES peaks. SEM image of this annealed sample (b). The island and flat part measurement points are also displayed in (b), and indicate the chosen positions for the AES spectra in (a), on a column and on the area between the columns.

There is a clear match of the both AES spectra in Fig. 3.5(a), indicating similar elemental composition on both columns and areas between the columns. The AES peaks in the energy ranges from $\sim 155$ to $\sim 280 \mathrm{eV}$ and from $\sim 484$ to $\sim 518 \mathrm{eV}$ correspond to the respective $\mathrm{Ru}$ and $\mathrm{O}$ surface elements, and their peak-to-peak amplitude corresponds to ruthenium oxide. ${ }^{322}$ It should be noted that we cannot exclude $\mathrm{S}$ and $\mathrm{C}$ surface 
contamination (see Fig. 3.5(a)), probably related to previous atmospheric exposure. Still, AES analysis indicates that the elemental composition of both columns and areas between the columns is the same.

X-ray diffraction patterns were recorded for all annealed samples and example spectra are presented in Fig. 3.6 for an as deposited sample (solid line) and a $20 \mathrm{~min}$ annealed sample at $400^{\circ} \mathrm{C}$ (dashed line). The magnetron sputtered $\mathrm{Ru}$ layer exhibits a hcp polycrystalline structure ${ }^{14,141,323}$ over the entire temperature range, with intensities reducing at higher temperatures due to $\mathrm{Ru}$ consumption during $\mathrm{RuO}_{2}$ growth. For $\mathrm{Ru}$, no angular dependence of the diffracted intensity is observed (not shown) apart from that induced by the illumination geometry, and as such a random orientation of crystallites is suggested. $\mathrm{RuO}_{2}$ peaks typical for rutile-like crystalline structure ${ }^{14}$ are detected, showing two types of diffraction patterns. One pattern is detected above $275^{\circ} \mathrm{C}$ and belongs to larger oriented crystallites, with (101) planes being inclined about $17 \mathrm{deg}$. with respect to the sample surface. Additionally to this pattern, another pattern is detected above $375^{\circ} \mathrm{C}$, which belongs to smaller randomly oriented crystallites.

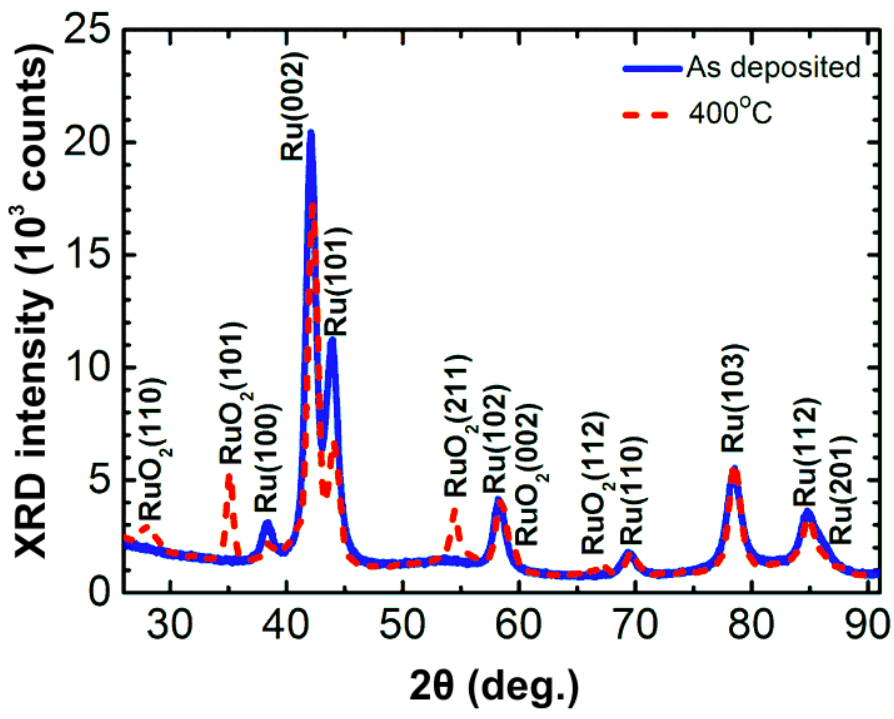

Fig. 3.6. X-ray diffraction patterns for an as deposited sample (solid line) and a 20 min annealed sample at $400^{\circ} \mathrm{C}$ (dashed line).

We selected the (002) and (101) diffraction peaks of $\mathrm{Ru}$ and the (110) and (101) diffraction peaks of $\mathrm{RuO}_{2}$ for further analysis due to their high intensity and due to the fact that they correspond to the crystals with orientation close to the oxide film growth. The crystallite sizes in the directions corresponding to $\mathrm{Ru}(002), \mathrm{Ru}(101), \mathrm{RuO}_{2}(110)$ and $\mathrm{RuO}_{2}(101)$ peaks were determined in the temperature range of $300-500^{\circ} \mathrm{C}$ using the Scherrer equation. ${ }^{301,302}$ The Scherrer constants used were 0.94 and 0.88 for $\mathrm{RuO}_{2}$ and 
$\mathrm{Ru}$, respectively. ${ }^{302}$ Fig. 3.7 (closed symbols) shows the respective $\mathrm{Ru}$ and $\mathrm{RuO}_{2}$ crystallite sizes. Also shown are the $\mathrm{Ru}$ and total $\mathrm{RuO}_{2}$ (summed low density and high density) thicknesses as determined by XRR measurements (open symbols). Comparing the closed pentagons and closed stars to the open triangles, it can be observed that the $\mathrm{Ru}(101)$ and $\mathrm{Ru}(002)$ crystallite sizes are in agreement with the Ru layer thickness within the error bars. For $\mathrm{RuO}_{2}(110)$, corresponding to the mentioned smaller crystallites, the crystallite size matches the XRR thickness of the 2D oxide film, as depicted by the closed rhombus and open circles, respectively. In contrast for $\mathrm{RuO}_{2}(101)$, corresponding to the mentioned larger crystallites, the crystallite size is about ten times larger than the total $\mathrm{RuO}_{2}$ layer thickness extracted from XRR analysis, as presented by the respective solid squares and open circles. This apparent inconsistency can be resolved when considering the already mentioned surface morphology of the samples. It appears that the $\mathrm{RuO}_{2}(101)$ crystallite size matches very well the average height of the surface $3 \mathrm{D}$ columns determined by AFM (see for example the sample annealed at $300^{\circ} \mathrm{C}$, the first closed square at Fig. 3.7 and Fig. 3.4(h)). Note that, accordingly, the average height of the columns is also more than ten times larger than the thickness of the thin oxide film (see for example the sample annealed at $300^{\circ} \mathrm{C}$, Fig. 3.4(h) and Fig. 3.7 (first circle)).

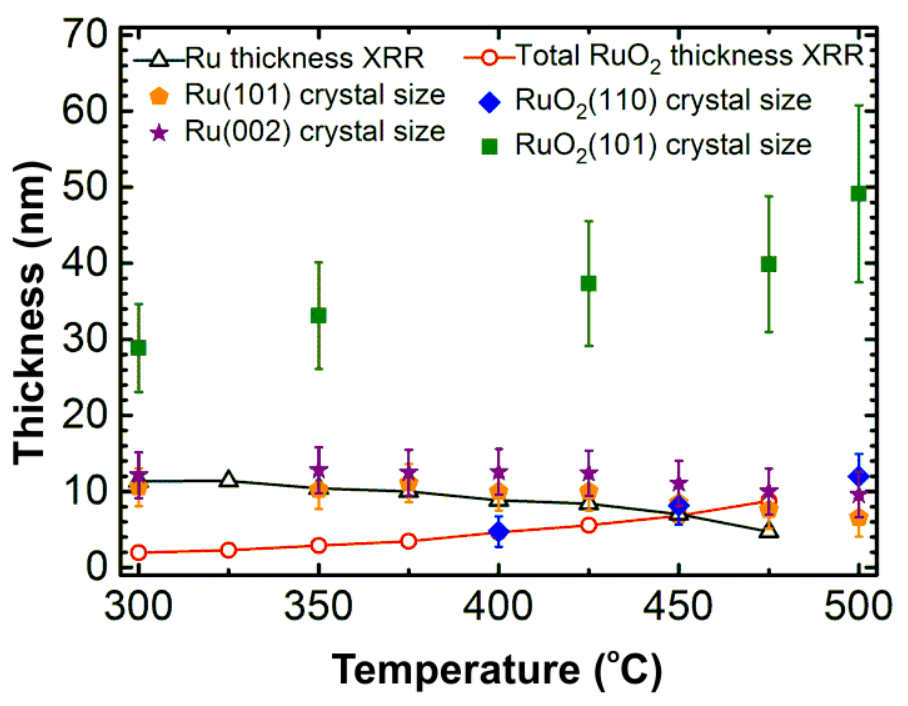

Fig. 3.7. Film thicknesses and crystal sizes as a function of annealing temperature for the samples annealed in the temperature range of $300-500^{\circ} \mathrm{C}$ for 20 minutes. The $\mathrm{Ru}$ and total $\mathrm{RuO}_{2}$ (summed low density and high density) thicknesses obtained from XRR are presented by open triangles and open circles, correspondingly. The crystal sizes corresponding to $\mathrm{Ru}(101), \mathrm{Ru}(002), \mathrm{RuO}_{2}(110)$, and $\mathrm{RuO}_{2}(101) \mathrm{XRD}$ peaks are presented by closed pentagons, closed stars, closed rhombus and closed squares, correspondingly. 
Below, we calculated the ratio of the consumed $\mathrm{Ru}$ to the formed $\mathrm{Ru}$ oxide taking into account both oxides, in the thin film and in the columns. From the AFM images, an effective volume for the $\mathrm{RuO}_{2}$ columns can be extracted after a geometric convolution correction. This $\mathrm{RuO}_{2}$ volume, divided by the AFM scan area, yields an "effective thickness", the value as if all the amount of $\mathrm{RuO}_{2}$ observed in the $3 \mathrm{D}$ columns would be distributed in a flat continuous layer. This is depicted in Fig. 3.8 (closed squares) together with the thin $\mathrm{Ru}$ oxide film thickness (solid circles). The similar growth of these two curves indicates that both oxides, the thin film and the columns, have similar volume growth rate, which is important for further discussion. Fig. 3.8 also shows reduction of the $\mathrm{Ru}$ layer thickness (open triangles) with annealing. When the $\mathrm{RuO}_{2}$ "effective thickness" determined from AFM is added to the $\mathrm{RuO}_{2}$ thickness obtained from XRR and then divided by the Ru loss, a ratio of 3.5 is achieved at low temperatures (Fig. 3.8, dashed line), consistent with the larger contribution of the low density Ru oxide layer growth at low temperatures. For the high temperatures, the ratio approaches the calculated value of 2.3 , consistent with the predominant high density $\mathrm{RuO}_{2}$ growth observed from XRR.

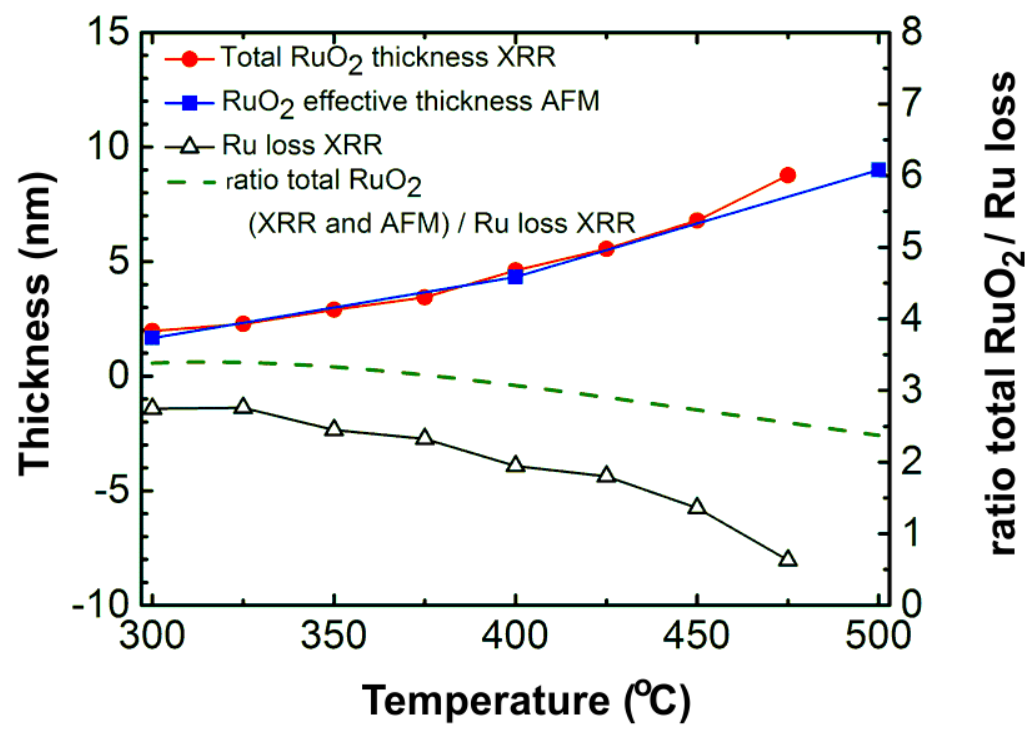

Fig. 3.8. Oxide thickness and $\mathrm{Ru}$ loss as a function of annealing temperature for $20 \mathrm{~min}$ annealed samples in the range of $300-500^{\circ} \mathrm{C}$. Total $\mathrm{RuO}_{2}$ thickness (summed low density and high density) obtained from XRR is presented by closed circles. The $\mathrm{RuO}_{2}$ "effective thickness" from AFM analysis is depicted by closed squares. The Ru loss from XRR is shown by open triangles. The ratio between total $\mathrm{RuO}_{2}$ (summed XRR and AFM amount) and $\mathrm{Ru}$ loss from XRR is represented by the dashed line. The accuracies of the layer thicknesses determined by XRR and AFM analysis are \pm 0.1 and $\pm 0.3 \mathrm{~nm}$, respectively. 
High resolution transmission electron microscopy (HR-TEM) showed more details on the formed structure, as depicted by the cross-section of a 20 minutes annealed $\mathrm{Ru}$ sample at $300^{\circ} \mathrm{C}$ (Fig. 3.9). First of all, it confirms the polycrystallinity of the Ru layer with different crystal orientations, as represented by the fast Fourier transforms (FFT) calculated from the square areas of the HR-TEM image (Fig. 3.9(a), insets 1 to 4). The thin ruthenium oxide film on top of polycrystalline Ru is visible (Figs. 3.9(a), 3.9(b) and 3.9(c)). Its thickness is in a very good agreement with the one obtained from XRR. Some crystalline parts in this ruthenium oxide layer are observed in Fig. 3.9(a). But, it is too thin to conclude if the crystalline part belongs to the ruthenium oxide layer itself or to the $\mathrm{Ru}$ layer which overwhelms the very thin top layer in the HR-TEM image. As illustrated in Fig. 3.9(b), the majority of the surface columns were detached from the surface during TEM preparation. However, it is well visible in Fig. 3.9(c) that the columns have rectangular- rather than spherical-like shape as imaged by AFM. They have an aspect ratio of about $1: 3$ on average (for the sample at $300^{\circ} \mathrm{C}$ ), demonstrating strongly anisotropic growth. The columns grow monocrystalline. HR-TEM at Fig. 3.9(d) resolves the (101) planes that are positioned at $115^{\circ}$ with respect to the longer side of the column. This side corresponds to (110) crystal plane, taking into account the rutile-like structure of the oxide. For this structure, (110) surface has the lowest energy, ${ }^{324}$ which explains why it is the most abundant surface of the observed columns.

It cannot be observed by TEM if the columns grow at the surface of $\mathrm{Ru}$ or at the surface of the ruthenium oxide thin layer. The columns could, in principle, grow as continuation of the Ru grains with the proper orientation. In that case, Ru would act as a seeding layer for the columns. However, the columns are typically a factor of ten larger in height compared to the oxide film thickness. If they would originate at the initial $\mathrm{Ru}$ surface, they would have already been detected by AFM for the sample annealed at $175^{\circ} \mathrm{C}$ (Fig. 3.4(b)). According to XRR, at this temperature, there is already a $0.85 \mathrm{~nm}$ low density thin film oxide formed at the surface, which is expected to be a closed layer. So, it is still most likely that the columns grow at the surface of the formed thin ruthenium oxide layer rather than the surface of Ru layer. Note that the volume growth rate of the columns is similar to the one of the thin oxide film as mentioned above. Obviously, both rates are limited by diffusion of atoms through the thin oxide film, ruthenium upwards and oxygen downwards. 


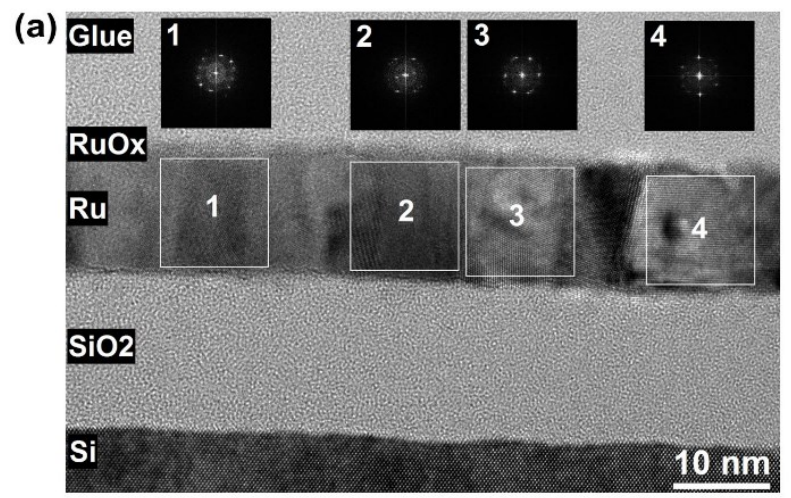

(b)

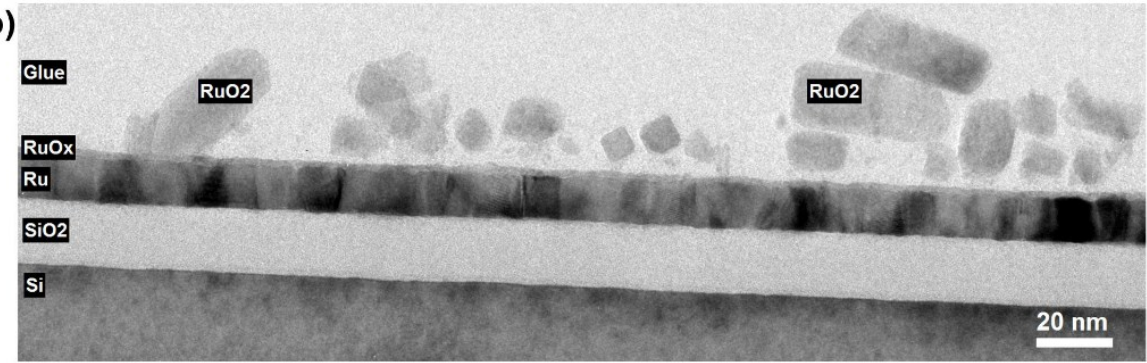

(c)

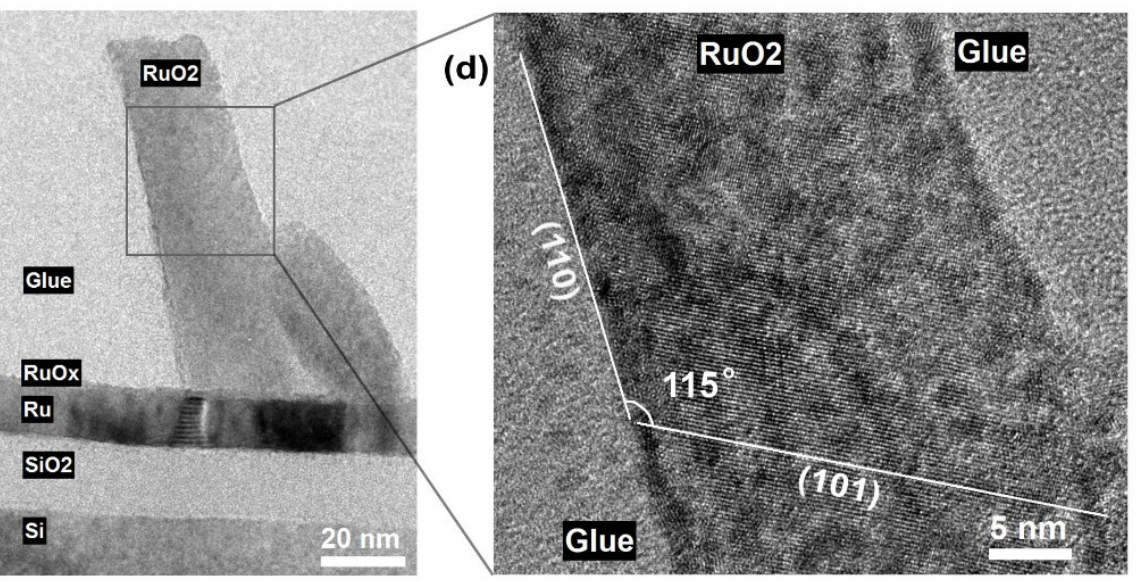

Fig. 3.9. Cross-sectional HR-TEM images of a 20 minutes annealed sample at $300^{\circ} \mathrm{C}$. Ru layer, region without $\mathrm{RuO}_{2}$ nano-columns (a). $\mathrm{Ru}$ layer with tilted and detached surface $\mathrm{RuO}_{2}$ nanocolumns (b). Rectangular-like tilted nano-columns attached to the film surface, showing the strongly anisotropic growth (c). Zoom view of the monocrystalline $\mathrm{RuO}_{2}$ column with the resolved (101) planes that go from side to side of the column (b). Note that the growth direction of the column is parallel to the (110) $\mathrm{RuO}_{2}$ planes, forming an angle of $115^{\circ}$ with the (101) planes. Fast Fourier transforms (FFT) calculated from the square areas of the HR-TEM image are depicted for the Ru layer (insets 1 to 4 in (a)). 
The remaining question is why the nano-columns prefer to grow in 3D mode at the surface of the ruthenium oxide layer and not to continue growing in the initial 2D thin film mode. We suggest that there are several factors contributing to the coexistence of the $2 \mathrm{D}$ and $3 \mathrm{D}$ growth. Oxidation of ruthenium film starts with the formation of a low density $\mathrm{RuO}_{\mathrm{x}}(2<\mathrm{x} \leq 3)$ layer. In the process of further oxidation, this low density oxide layer is always present on top but is limited to a maximum of $3 \mathrm{~nm}$. The reduced supply of ruthenium atoms that have to diffuse through the ruthenium oxide layer towards the surface and the unlimited supply of oxygen at the surface are the possible reasons why the oxygen rich low density oxide layer is initially formed at the surface. Below this layer, a higher density stoichiometric $\mathrm{RuO}_{2}$ will grow during further oxidation. Up to a temperature of $\sim 375^{\circ} \mathrm{C}$, the oxide film seems to remain quasi-amorphous, probably to keep the minimum energy interface with ruthenium. At about $200^{\circ} \mathrm{C}$, because of the rather high mobility of $\mathrm{Ru}$ or $\mathrm{Ru}-\mathrm{O}$ precursors at the surface, ${ }^{224}$ the first crystalline $\mathrm{RuO}_{2}$ nuclei will form at the very surface, with Ru being supplied from the bottom via diffusion through the oxide film. And, since the $\mathrm{RuO}_{\mathrm{x}}$ top layer with a rather disordered structure does not support growth of a crystalline structure along the surface, $\mathrm{RuO}_{2}$ crystallites will continue growing in the vertical direction. We detect the first crystalline columns at $275^{\circ} \mathrm{C}$ when the oxide film still stays quasi-amorphous. At temperatures above $375^{\circ} \mathrm{C}$ this film starts to crystallize, turning polycrystalline. At that moment, the columns already become stable and continue growing at the expense of all the ruthenium reaching the surface.

\subsection{Conclusions}

In summary, a mixed $2 \mathrm{D}$ and $3 \mathrm{D}$ growth of $\mathrm{Ru}$ oxide has been experimentally observed for thermally oxidized polycrystalline $\mathrm{Ru}$ thin films. Below a threshold temperature of $200^{\circ} \mathrm{C}$ there is approximately one monolayer of thin film low density $\mathrm{RuO}_{\mathrm{x}}(2<\mathrm{x} \leq 3)$ on the $\mathrm{Ru}$ surface formed. Above $200^{\circ} \mathrm{C}, \mathrm{RuO}_{2}$ nano-columns are detected on the surface, growing in size with temperature and annealing duration. Simultaneously with the growth of the columns, sub-surface oxidation continues. The low density oxide film is followed by the formation of a near bulk density $\mathrm{RuO}_{2}$ thin layer. The total amount of oxide formed, including 2D films and 3D nano-columns, is consistent with the reduction of the $\mathrm{Ru}$ layer thickness.

\section{Acknowledgements}

This work is part of the research programme 'Controlling photon and plasma induced processes at EUV optical surfaces (CP3E)' of the 'Stichting voor Fundamenteel Onderzoek der Materie (FOM)', which is financially supported by the 'Nederlandse Organisatie voor Wetenschappelijk Onderzoek (NWO)'. The CP3E programme is co- 
Chapter 3

financed by Carl Zeiss SMT and ASML. We also acknowledge financial support from Agentschap NL (EXEPT project). 


\title{
4. Determination of oxygen diffusion kinetics during thin film ruthenium oxidation
}

\begin{abstract}
In situ X-ray reflectivity was used to reveal oxygen diffusion kinetics for thermal oxidation of polycrystalline ruthenium thin films and accurate determination of activation energies for this process. Diffusion rates in nanometer thin $\mathrm{RuO}_{2}$ films were found not to be constant, and to decrease with growing oxide thickness. Arrhenius plots of the diffusion rates suggested a single diffusion process occurring for each $\mathrm{RuO}_{2}$ thickness with a single activation energy associated, which increased gradually from about 2.1 to $2.4 \mathrm{eV}$ in the oxide thickness range of 3.0 to $4.5 \mathrm{~nm}$. Further exploration of the Arrhenius pre-exponential factor for the diffusion process revealed that oxidation of polycrystalline ruthenium seems to join the class of processes that obey the MeyerNeldel rule.
\end{abstract}




\subsection{Introduction}

Ruthenium oxide $\left(\mathrm{RuO}_{2}\right)$ presents several attractive properties such as low resistivity, ${ }^{15}$ high thermal stability, ${ }^{16}$ good diffusion barrier capability, ${ }^{17}$ excellent catalytic performance, ${ }^{12,13}$ and easy patterning. ${ }^{18}$ These interesting properties can make $\mathrm{RuO}_{2}$ a suitable candidate for a large number of applications. For instance, $\mathrm{RuO}_{2}$ can be used as oxidation catalyst in heterogeneous catalysis and electrocatalysis, ${ }^{14}$ as bottom gate electrode of oxide dielectric capacitors in dynamic random access memories (DRAMs), ${ }^{6}$ or as protective capping layer in extreme ultraviolet lithography (EUVL). ${ }^{7,9,10}$ In the last two applications, oxygen diffusion towards deeper layers is known to be one of the main threats for functionality. The fabrication of high-K dielectric capacitors is performed at high temperatures and in an oxidative environment. Under such extreme conditions, the poly-Si plug attached to the gate electrode can be oxidized with a subsequent dielectric thickness increase, which then results in a marked reduction of the effective dielectric constant of the capacitor with negative implications for its performance. ${ }^{6}$ Under EUV radiation, the cracking of water vapor potentially present in the system will induce oxidation of the Si/Mo multilayer mirrors resulting in a drop in reflectivity with a consequently decrease of the optics lifetime. ${ }^{9,41}$ In both cases, a protection barrier for oxygen diffusion is necessary, for which $\mathrm{RuO}_{2}$ has been considered as a good candidate. However, there is a lack of knowledge on diffusion kinetics, diffusion constants and activation energies for oxygen diffusion in $\mathrm{RuO}_{2}$ thin films that would allow determination of the degradation rate of the structures due to oxidation.

The limited availability of data on diffusion constants in nanometer thin films is due to the very intricate measurement of diffusion coefficients in systems with short diffusion lengths. ${ }^{269}$ The commonly used techniques for this purpose, such as transmission electron microscopy and X-ray photoelectron spectroscopy, do not allow to determine small changes in the structures with sufficient precision. To tackle this problem, in our recent work, ${ }^{325}$ in situ hard X-ray reflectivity measurements were used for monitoring thermal oxidation of $\mathrm{Ru}$ thin films. This allowed accurate determination of the changes of the in-depth electron density distribution during the thin film growth. There, we reported about co-existence of 3D surface and 2D sub-surface oxidation. In this chapter, we focus on the determination of diffusion kinetics for the sub-surface oxidation process of $\mathrm{Ru}$ thin films. Diffusion rates and activation energies for oxygen diffusion through a few nanometer thick ruthenium oxide layers have been accurately determined. 


\subsection{Experimental}

Ten nanometer thick polycrystalline Ru films were deposited by DC magnetron sputtering at room temperature onto natively oxidized super-polished Si substrates in an UHV setup with base pressure $<1 \times 10^{-8}$ mbar. To prevent intermixing of $\mathrm{Ru}$ and $\mathrm{Si}$ during annealing, and resulting complications in X-ray reflectivity data analysis, a $15 \mathrm{~nm}$ $\mathrm{SiO}_{2}$ diffusion barrier was deposited on top of the $\mathrm{Si}$ wafer before $\mathrm{Ru}$ deposition. All layer thicknesses were monitored using quartz mass balances and used as initial fit parameters for XRR analysis.

In situ monitoring of $\mathrm{Ru}$ oxidation during annealing was conducted with $\mathrm{X}$-ray reflectivity (XRR) measurement using a PANalytical Empyrean X-ray diffractometer $(\mathrm{Cu}-\mathrm{K} \alpha$ radiation, $0.154 \mathrm{~nm})$, equipped with an Anton Paar thermal stage. ${ }^{273}$ Before annealing, the sample position was aligned with respect to the impinging X-ray beam and a reference XRR spectrum was recorded. Then, the sample was heated to a predefined temperature, and a sample realignment was carried out to correct for thermal expansion and possible misalignment. Subsequently, XRR scans were repeatedly performed during annealing.

The deposited $\mathrm{Ru}$ films were annealed at different temperatures for different amounts of time depending on temperature. GenX software was used for XRR data analysis. ${ }^{294}$ A layered model of the structure was simulated using GenX, and the thickness, roughness and density of each of the layers in the model were varied using a genetic algorithm in order to minimize the differences between model simulations and experimental data. Layer thicknesses and densities were determined with accuracies of $\pm 0.1 \mathrm{~nm}$ and $\pm 0.3 \mathrm{~g} \cdot \mathrm{cm}^{-3}$, respectively. Ruthenium oxide thicknesses were monitored in time to obtain diffusion constants and activation energies.

X-ray diffraction (XRD) patterns were also recorded using a PANalytical Empyrean X-ray diffractometer $(\mathrm{Cu}-\mathrm{K} \alpha$ radiation, $0.154 \mathrm{~nm})$.

\subsection{Results and discussion}

Fig. 4.1 presents an example of the measured and simulated XRR data for (a) an as deposited and (b) annealed sample at $400^{\circ} \mathrm{C}$ for 20 minutes. The models consisted of a $\mathrm{Si}$ substrate, a $\mathrm{SiO}_{2}$ layer, a $\mathrm{Ru}$ layer and one or two $\mathrm{RuO}_{2}$ layers, as shown in the insets of Fig. 4.1. 


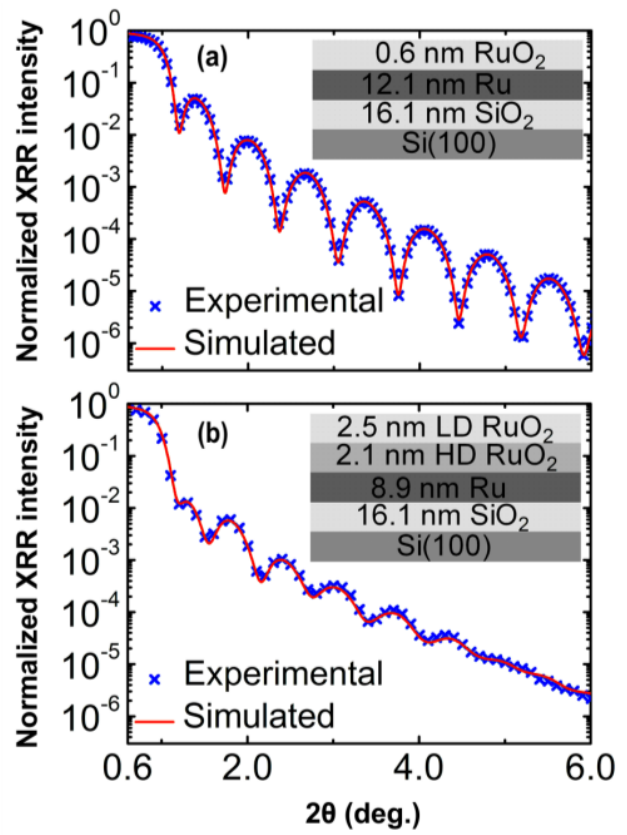

Fig. 4.1. Experimental (crosses) and simulated (line) specular X-ray reflectivity data for (a) an as deposited sample and (b) a sample annealed at $400^{\circ} \mathrm{C}$ for $20 \mathrm{~min}$. Top-right (a) and (b) insets show the layered models used for each simulation. $\operatorname{LD}\left(0.7 \rho_{\text {bulk }}\right)$ and $\mathrm{HD}\left(\rho_{\text {bulk }}\right)$ denote low and high density, respectively.

The XRR data for the as deposited sample could be modelled using a single thin surface oxide layer with a density of $6.0 \pm 0.3 \mathrm{~g} \cdot \mathrm{cm}^{-3}$, which is somewhat lower than the bulk density of $\mathrm{RuO}_{2}, 6.8 \mathrm{~g} \cdot \mathrm{cm}^{-3}$. The structure model is depicted in Fig. 4.1(a). In the range of temperatures from 150 to $350^{\circ} \mathrm{C}$, the oxide layer thickness increased from $0.7 \pm 0.1$ to $2.4 \pm 0.1 \mathrm{~nm}$, respectively, with the density being $6.0 \pm 0.3 \mathrm{~g} \cdot \mathrm{cm}^{-3}$. Above about $350^{\circ} \mathrm{C}$, however, XRR data could not be modelled anymore by a single surface oxide layer. To find an appropriate model for the oxide, a test model was created. For a sample annealed during $12 \mathrm{~h}$ at $400^{\circ} \mathrm{C}$, the oxide layer was subdivided into 9 thinner oxide layers of $1 \mathrm{~nm}$ each, with the density for each sub-layer being varied by the genetic algorithm from 5 to $7.5 \mathrm{~g} \cdot \mathrm{cm}^{-3}$. The other layers remained as in the initial model and were fitted in the conventional way. The result of the fit showed a clear separation of the oxide into two parts. The top three sub-layers had consistently lower density of about $70 \%$ compared to the bottom six sub-layers having close to the bulk $\mathrm{RuO}_{2}$ density. So above $350^{\circ} \mathrm{C}$ it was necessary to use a 2-layer instead of 1-layer oxide model to better describe the oxidation process, a low density (LD) $\mathrm{RuO}_{2}$ layer on top of a high density (HD) $\mathrm{RuO}_{2}$ layer, with LD and HD oxide densities being $5.3 \pm 0.3$ and $6.8 \pm 0.3 \mathrm{~g} \cdot \mathrm{cm}^{-3}$, respectively (see (Fig. 4.1(b)). The low density $\mathrm{RuO}_{2}$ quickly saturated with annealing time at a thickness of about $3 \mathrm{~nm}$ while the high density $\mathrm{RuO}_{2}$ continued to grow as shown by the 
open and closed symbols in Fig. 4.2(a), respectively. Note that the reason for the origin of the low density oxide is not clear and needs further investigation. But since the high density $\mathrm{RuO}_{2}$ was the only growing oxide species, the oxygen diffusion kinetics was studied monitoring the thickness of this layer.

For this study, the temperatures had to be carefully selected. First of all, only above $350^{\circ} \mathrm{C} \mathrm{XRR} \mathrm{analysis} \mathrm{suggested} \mathrm{formation} \mathrm{of} \mathrm{close} \mathrm{to} \mathrm{bulk} \mathrm{density} \mathrm{RuO}_{2}$. At the same time, temperatures higher than $400^{\circ} \mathrm{C}$ provoked strong surface morphology changes, observed by the drop of the XRR signal, probably due to the pronounced formation and advanced growth of surface nano-columns above these temperatures. ${ }^{325}$ Such morphology changes strongly reduce the apparent optical contrast in the XRR, which lowers accuracy of XRR analysis. For this reason, in situ XRR measurements during annealing were done in the carefully selected range of temperatures, at 380,390 , and $400^{\circ} \mathrm{C}$. The small range of temperatures and the increment of only 10 degrees ensured minimum possible structural differences in the growing oxides at different temperatures. Resolving changes in diffusion rates with this small increment in temperature became possible due to the high accuracy of the oxide thickness determination with XRR measurements. Furthermore, before the experiment the samples were pre-annealed at $400^{\circ} \mathrm{C}$ for $2 \mathrm{~h}$. At this step, the low density oxide layer is saturated at a thickness of approximately $3 \mathrm{~nm}$ and there is a layer of about $2.8 \mathrm{~nm}$ near bulk $\mathrm{RuO}_{2}$ formed (see Fig. 4.2(a)). The pre-annealing step allowed us to avoid non-diffusion limited or concentration dependent diffusion processes that shortly occur at the initial stages of surface oxidation which also strongly depend on the temperature of annealing.

To exclude a possible effect of changing structure with time for different temperatures, diffusion rates were determined at fixed oxide thicknesses, rather than fixed annealing time, for different temperatures. So, here initially an assumption was made that for every oxide thickness the structure and composition of the oxide were identical for different temperatures in the range between 380 and $400^{\circ} \mathrm{C}$. The high density and the low density $\mathrm{RuO}_{2}$ thicknesses determined from XRR analysis are plotted in Fig. 4.2(a) as a function of annealing time for annealing temperatures of 380, 390 and $400^{\circ} \mathrm{C}$. From the evolution of the high density $\mathrm{RuO}_{2}$ thicknesses, diffusion rates for oxygen diffusion in $\mathrm{RuO}_{2}$ were determined under the reasonable assumption of diffusion limited oxide growth, ${ }^{253}$ as follows:

$$
D=\frac{d z^{2}}{d t},
$$

where $z$ is the HD oxide thickness and $t$ is the annealing time. A second degree polynomial was applied to smoothen the raw data and to be able to take derivative according to Eq. (4.1). The calculated diffusion rates are plotted as function of HD oxide thickness in Fig. 4.2(b). 

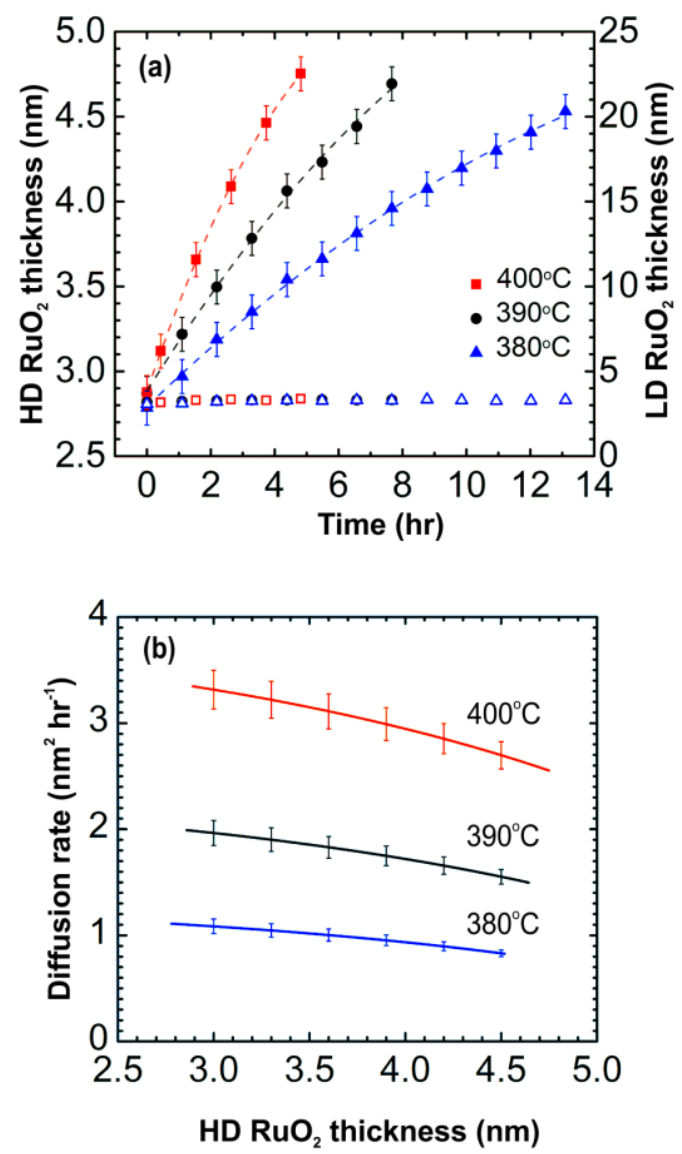

Fig. 4.2. High density (HD) $\mathrm{RuO}_{2}$ thickness (closed symbols) and low density (LD) $\mathrm{RuO}_{2}$ thickness (open symbols) as function of annealing time (a), and diffusion rate for oxygen diffusion in $\mathrm{RuO}_{2}$ as a function of $\mathrm{HD} \mathrm{RuO}_{2}$ thickness (b), for samples annealed at $380^{\circ} \mathrm{C}$ (triangles), $390^{\circ} \mathrm{C}$ (circles) and $400^{\circ} \mathrm{C}$ (squares). $1 \mathrm{~nm}^{2} \mathrm{~h}^{-1}$ corresponds to $2.78 \cdot 10^{-18} \mathrm{~cm}^{2} \mathrm{~s}^{-1}$. Dashed lines for the $\mathrm{HD} \mathrm{RuO}_{2}$ are guides for the eye. Note that all the samples were pre-annealed for $2 \mathrm{~h}$ at $400^{\circ} \mathrm{C}$ before in situ XRR measurements resulting in the initial $2.8 \pm 0.1 \mathrm{~nm} \mathrm{HD} \mathrm{RuO}$ film. A second degree polynomial was applied to smoothen the raw data and to be able to take derivative according to Eq. (4.1) for determining the oxygen diffusion rates.

Remarkably, Fig. 4.2(b) shows a general reduction of the diffusion rates with growing oxide thickness, indicating that there is a gradual change in the oxide structure with thickness that can affect diffusion. To determine oxygen diffusion kinetics and to check how it depends on $\mathrm{RuO}_{2}$ thickness, we plotted the dependence of the diffusion rates on temperature at different oxide thicknesses. In Fig. 4.2(b) there is a range of oxide thicknesses between 3.0 to $4.5 \mathrm{~nm}$ where diffusion rate data is available for all investigated temperatures. Plotting $\ln D$ versus $1 / k_{B} T$ suggests a linear dependence for 
all oxide thicknesses within the explored range (Fig. 4.3(a)). This seems in agreement with the Arrhenius-type temperature behavior typical for many thermally induced processes: 253

$$
D=D_{0} e^{-E_{a} / k_{B} T}, \ln D=\ln D_{0}-\frac{E_{a}}{k_{B} T},
$$

where $D_{0}$ is the pre-exponential factor, $E_{a}$ is the activation energy, $T$ is the absolute temperature, and $k_{B}$ is the Boltzmann constant. This indicates that in spite of the reducing diffusion rates with growing oxide thickness, for every particular oxide thickness, the diffusion process seems to be governed by a single diffusion mechanism with a certain activation energy associated. This also confirmed our initial suggestion that for each oxide thickness the structure and composition of the oxide were identical independent of what temperature was used to achieve that particular oxide thickness.

We should note that only a limited number of data points were used in this experiment to support the drawn conclusions (Fig. 4.3(a)). This is a result of the narrow temperature range selected in view of the peculiar growth properties of $\mathrm{RuO}_{2}$ outside this range as described above. Nevertheless, the accuracy of thickness, and especially, the relative thickness change, determination with in situ XRR allowed us to make reliable conclusions. Fig. 4.3(b) shows the activation energy as a function of the growing $\mathrm{RuO}_{2}$ thickness, determined from the slopes of the lines in Fig. 4.3(a).

The activation energy seems to increase from $2.09 \pm 0.08$ to $2.35 \pm 0.08 \mathrm{eV}$, while the thickness of the $\mathrm{RuO}_{2}$ increases from 3.0 to $4.5 \mathrm{~nm}$. This explains the significant decrease of the diffusion rates with the growing oxide thickness. Taking into account the fact that no change in the density of the growing oxide was detected with the XRR measurements, we looked for other possible factors that could explain such increase in the activation energy. One of them is the crystalline structure of the formed layers.

X-ray diffraction measurements showed that $\mathrm{Ru}$ layer exhibited a hcp polycrystalline structure over the entire temperature range. For $\mathrm{RuO}_{2}$, a typical pattern of a rutile-like crystalline structure was detected. The crystallite sizes of $\mathrm{Ru}$ and $\mathrm{RuO}_{2}$ were determined for all temperature range using the Scherrer equation. ${ }^{302}$ The crystal size of Ru reduced during annealing in the direction perpendicular to the sample surface. It changed from about 7.5 to $6.2 \mathrm{~nm}$ due to $\mathrm{Ru}$ consumption to form $\mathrm{RuO}_{2}$. Opposite to $\mathrm{Ru}$ crystals, $\mathrm{RuO}_{2}$ crystals seemed to increase in size. Although we could not determine accurately their size due to their weak diffraction signal, this increase in size was consistent with the increasing thickness of the oxide film from about $3 \mathrm{~nm}$ to $4.5 \mathrm{~nm}$ determined by X-ray reflectivity. In view of the polycrystalline structure of the layers, it is very likely that diffusion in our system is dominated by the grain boundary diffusion. Usually, grain boundary diffusion follows Arrhenius-type temperature dependence like lattice diffusion. So, we presume that the activation energy measured in the studied 
system is predominantly determined by the grain boundary diffusion. If this is the case, the increase in the activation energy observed in our system should be related to the evolving grain boundary configuration in the polycrystalline $\mathrm{RuO}_{2}$ film that could obviously occur due to the observed growth of crystallites. Nevertheless, further detailed studies are necessary to confirm this suggestion and to determine what changes in the configuration of the grain boundaries do occur.
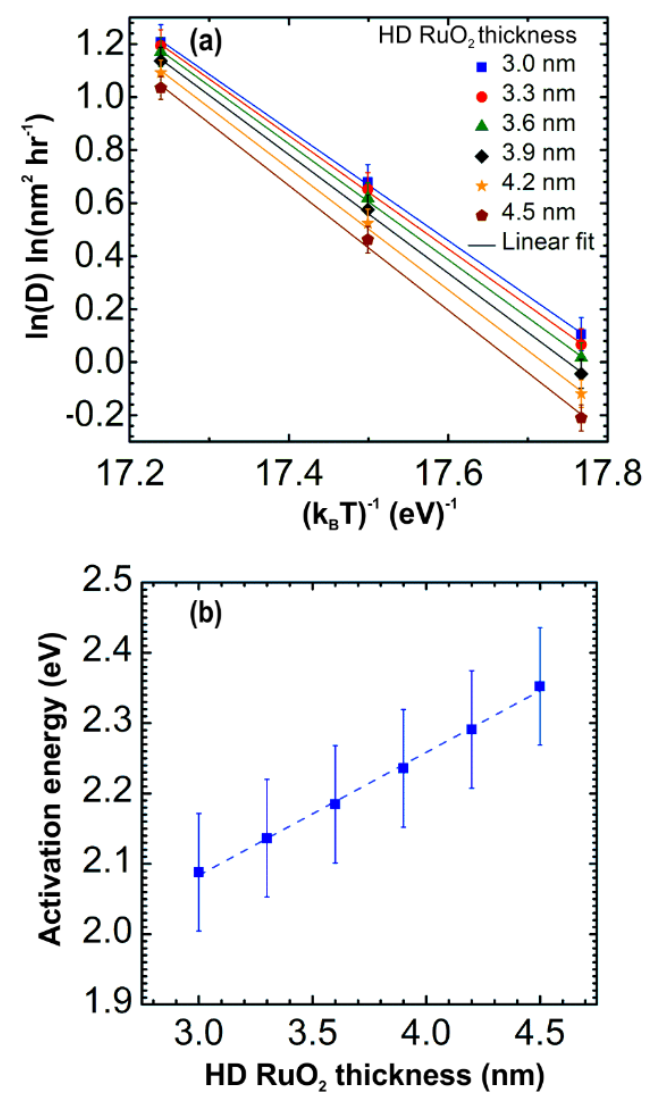

Fig. 4.3. (a) Natural logarithm of the diffusion rates as a function of $1 / k_{B} T$ for high density (HD) $\mathrm{RuO}_{2}$ thicknesses from 3.0 to $4.5 \mathrm{~nm} .1 \mathrm{~nm}^{2} \mathrm{~h}^{-1}$ corresponds to $2.78 \cdot 10^{-18} \mathrm{~cm}^{2} \mathrm{~s}^{-1}$. The lines are the linear fits to the data, being in agreement with the Arrhenius equation (Eq. (4.2)). (b) Activation energy for oxygen diffusion in $\mathrm{RuO}_{2}$ as function of high density (HD) $\mathrm{RuO}_{2}$ thickness. The error bars displayed in figure (b) represent $\sim 70 \%$ confidence levels for the activation energy, and are based on the calculations presented in Appendix A. The dashed line is a guide for the eye.

In view of the observed change in the activation energy during annealing, it was also important to check the dependency of the Arrhenius pre-exponential factor on the activation energy. This dependency is observed in some systems with thermally activated processes that show an Arrhenius type behavior, ${ }^{255}$ and recently proven not to depend on 
the way the activation energy is varied. ${ }^{254,326,327}$ The Arrhenius pre-exponential factor was calculated according to Eq. (4.2) for each oxide thickness. Plotting the natural logarithm of this pre-exponential factor versus the activation energy obtained for each oxide thickness shows a linear trend (Fig. 4.4). This indicates that in the diffusion process studied, not only the diffusion rate seems to depend exponentially on the activation energy, but also the pre-exponential factor seems to be exponentially dependent on the activation energy. This suggests that the process of ruthenium oxidation obeys the empirical Meyer-Neldel rule (MNR), ${ }^{255}$ which generally correlates the activation energy $E_{a}$ and the logarithm of the Arrhenius pre-exponential factor $D_{0}$ as

$$
\ln D_{0}=a+b \cdot E_{a}
$$

where $a$ and $b$ are constants. The $1 / b$ value corresponds to the Meyer-Neldel energy.

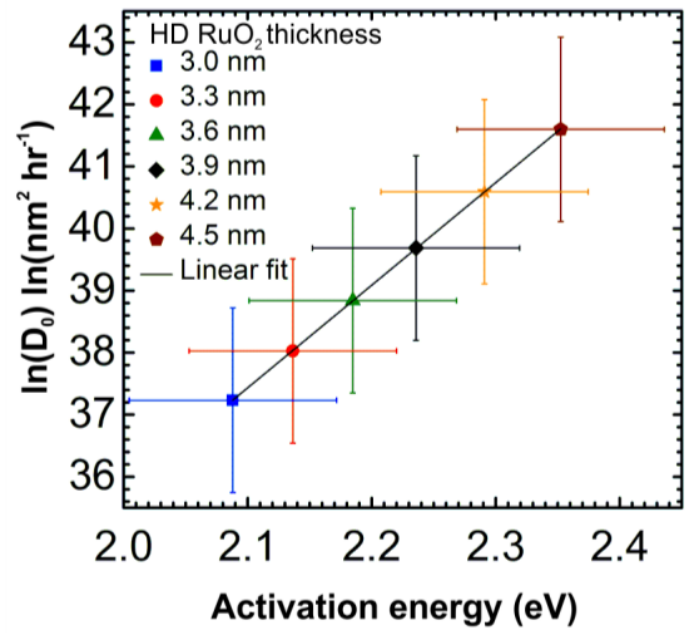

Fig. 4.4. Natural logarithm of the Arrhenius pre-exponential factor as a function of the activation energy obtained for different high density $\mathrm{RuO}_{2}$ thicknesses between 3.0 to $4.5 \mathrm{~nm} .1 \mathrm{~nm}^{2} \mathrm{~h}^{-1}$ corresponds to $2.78 \cdot 10^{-18} \mathrm{~cm}^{2} \mathrm{~s}^{-1}$. The solid line represents the linear fit to the data according to the Meyer-Neldel equation (Eq. (4.3)). From the slope of the line a Meyer-Neldel energy of $0.06 \pm 0.04$ $\mathrm{eV}$ is obtained. Note that the error bars indicate $\sim 70 \%$ confidence levels for both activation energies and pre-exponential factors, and are based on the calculations shown in Appendix A.

In effect, the increase of the pre-exponential factor when the activation energy is increased according to the Eq. (4.3), partially compensates for the decrease in the activation factor $e^{-E_{a} / k_{B} T}$ in the Arrhenius equation. This can be interpreted in general terms for diffusion processes as increasing the number of paths to the activated state with the increase of the activation energy. In our experiment, the effect is small compared to the effect of the activation energy on the activation factor that prevails the effect on the general diffusion rate. Still, it is significant and effectively can mean that the diffusion property may depend on more than one mechanism. Further research is needed to explain 
physics of this phenomenon as well as to determine up to what thicknesses of $\mathrm{RuO}_{2}$ the rule will be valid. Given the sensitivity and accuracy of the used method, it is also attractive to investigate oxygen diffusion kinetics at other stages of Ru oxidation. This especially concerns the earlier stages of oxidation where only low density oxide is formed. At the advanced stages of oxidation where larger crystals are formed, the lattice (volume) diffusion through $\mathrm{RuO}_{2}$ crystals can become the limiting factor for oxygen diffusion. However, in the latter case, the observed deterioration of the surface morphology presents a complicating factor for the used technique.

\subsection{Conclusions}

In situ X-ray reflectivity was used to reveal diffusion kinetics of inter-diffusion process for polycrystalline ruthenium thin film oxidation. This technique allowed accurate determination of diffusion constants and activation energies for this process. It was found that diffusion rates in several nanometer thick $\mathrm{RuO}_{2}$ films were not constant with oxide thickness. A gradual reduction in diffusion rate was observed with growing oxide thickness. However, for each particular $\mathrm{RuO}_{2}$ thickness, the diffusion process seemed to be governed by a single diffusion mechanism with a certain activation energy associated, following a linear Arrhenius dependence. In the considered range of $\mathrm{RuO}_{2}$ thicknesses from 3.0 to $4.5 \mathrm{~nm}$, the activation energy for interdiffusion increased from about 2.1 to $2.4 \mathrm{eV}$. We observed no detectable change in the density of the oxide film and suggested, evolving grain boundary configuration in the polycrystalline $\mathrm{RuO}_{2}$ as a possible mechanism for the slowing interdiffusion. Further exploration seemed to reveal an exponential dependence of the pre-exponential factor on the activation energy, which suggests that the process of ruthenium oxidation at the studied stage seems to obey the empirical Meyer-Neldel rule. And although the effect is small compared to the effect of the activation energy on the activation factor, it is present and needs further investigation to explain the physics behind this phenomenon. Also, further research is needed to determine oxidation kinetics of $\mathrm{Ru}$ at other stages of $\mathrm{RuO}_{2}$ formation.

\section{Acknowledgments}

This work is part of the research programme 'Controlling photon and plasma induced processes at EUV optical surfaces (CP3E)' of the 'Stichting voor Fundamenteel Onderzoek der Materie (FOM)', which is financially supported by the 'Nederlandse Organisatie voor Wetenschappelijk Onderzoek (NWO)'. The CP3E programme is cofinanced by Carl Zeiss SMT and ASML. We also acknowledge financial support from Agentschap NL (EXEPT project). 


\section{Appendix A. Determination of the uncertainties of the activation energies and pre-exponential factors for oxygen diffusion in $\mathrm{RuO}_{2}$ thin films}

To estimate the uncertainties of both activation energies and pre-exponential factors for oxygen diffusion through high density (HD) $\mathrm{RuO}_{2}$ thin films presented in Figs. 4.3(b) and 4.4 (Chapter 4), we performed the following data analysis. In addition to the experiments carried out in the temperature range between 380 and $400^{\circ} \mathrm{C}$, extra experiments were conducted at $350^{\circ} \mathrm{C}$. Fig. A. 1 shows the Arrhenius plots for $\mathrm{HD}_{\mathrm{RuO}}$ thicknesses of $3.0 \mathrm{~nm}(\mathrm{a})$, and $3.3 \mathrm{~nm}$ (b). It should be noted that due to the very low diffusion rates $\left(\sim 0.18 \mathrm{~nm}^{2} \mathrm{~h}^{-1}\right)$ at $350^{\circ} \mathrm{C}$, both activation energies $\left(E_{a}\right)$ and preexponential factors $\left(D_{0}\right)$ can only be evaluated in a limited thickness range (from 3.0 to $3.3 \mathrm{~nm}$ ) that overlaps with the experiments carried out at higher temperatures. Fits to the data according to the Arrhenius equation are displayed in Figs. A.1(a) and A.1(b) (solid lines).

(a)

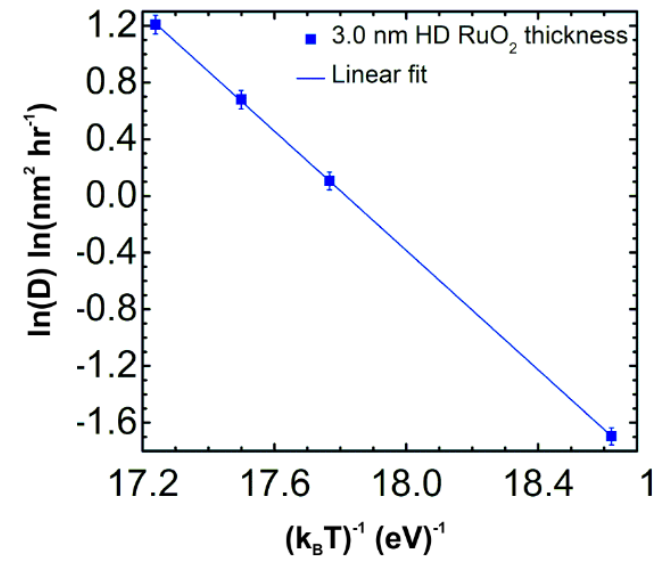

(b)

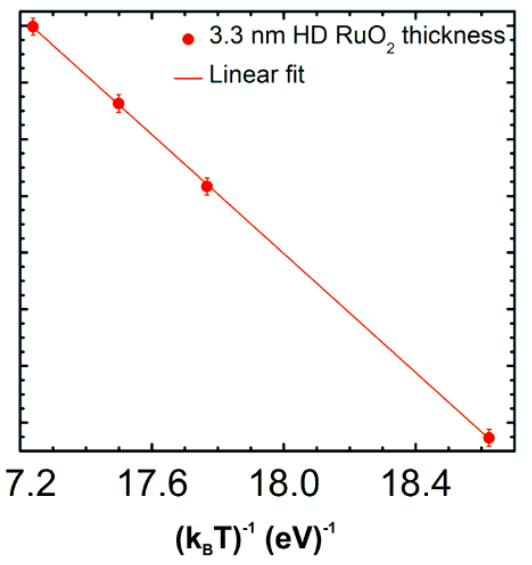

Fig. A.1. Natural logarithm of the diffusion rates as a function of $1 / k_{B} T$ for high density (HD) $\mathrm{RuO}_{2}$ thicknesses of $3.0 \mathrm{~nm}$ (a), and $3.3 \mathrm{~nm}$ (b). $1 \mathrm{~nm}^{2} \mathrm{~h}^{-1}$ corresponds to $2.78 \cdot 10^{-18} \mathrm{~cm}^{2} \mathrm{~s}^{-1}$. The lines in (a) and (b) are the linear fits to the data. 
A standard least-squares fitting of $\ln D=\ln D_{0}-E_{a} / k_{B} T$ with 2 free parameters $E_{a}$ and $\ln D_{0}$, including a standard error propagation analysis of the statistical errors in $\ln D$, is carried out on the data presented in Fig. A.1. ${ }^{328}$ Due to the limited number of experimental points used to fit a linear trend, the standard deviations obtained by the least-squares fitting, have to be multiplied according to the statistical $t$-distribution factor of $\sim 1.4$ (70\% confidence for 2 degrees of freedom) ${ }^{329}$ Uncertainties of $\pm 0.084 \mathrm{eV}$ for $E_{a}$, and of $\pm 1.5 \ln \left(\mathrm{nm}^{2} \mathrm{~h}^{-1}\right)$ for $\ln D_{0}$ are obtained, independently for both datasets. These uncertainties are based on data taken in the oxide thickness range from 3.0 to $3.3 \mathrm{~nm}$, and are expected to serve as good approximations for the errors in the oxide thickness range from 3.0 to $4.5 \mathrm{~nm}$. As such, these uncertainties are used in all datasets presented in Figs. 4.3(b) and 4.4.

\section{Acknowledgments}

We are grateful to Professor Diederik Depla (Ghent University) for essential discussions on statistical data analysis. 


\title{
5. In vacuo growth studies of $\mathrm{Ru}$ thin films on $\mathrm{Si}$, $\mathrm{SiN}$ and $\mathrm{SiO}_{2}$ by high-sensitivity low energy ion scattering
}

\begin{abstract}
In vacuo high-sensitivity low energy ion scattering (HS-LEIS) has been used to investigate the initial growth stages of DC sputtered $\mathrm{Ru}$ on top of $\mathrm{Si}, \mathrm{SiN}$ and $\mathrm{SiO}_{2}$. The high surface sensitivity of this technique allowed an accurate determination of surface coverages and thicknesses required for closing the Ru layer on all three substrates. The $\mathrm{Ru}$ layer closes (100\% Ru surface signal) at about 2.0, 3.2 and $4.7 \mathrm{~nm}$ on top of $\mathrm{SiO}_{2}$, $\mathrm{SiN}$ and $\mathrm{Si}$, respectively. In-depth $\mathrm{Ru}$ concentration profiles can be reconstructed from the $\mathrm{Ru}$ surface coverages when considering an error function like model. The large intermixing ( $4.7 \mathrm{~nm}$ ) for the $\mathrm{Ru}-\mathrm{on}-\mathrm{Si}$ system is compared to the reverse system (Si-on$\mathrm{Ru}$ ), where only $0.9 \mathrm{~nm}$ intermixing occurs. The difference is predominantly explained by the strong $\mathrm{Si}$ surface segregation that is observed for Ru-on-Si. This surface segregation effect is also observed for $\mathrm{Ru}$-on-SiN, but is absent for $\mathrm{Ru}$-on-SiO 2 . For this last system, in vacuo HS-LEIS analysis revealed surface oxygen directly after deposition, which suggests an oxygen surface segregation effect for $\mathrm{Ru}-\mathrm{on}-\mathrm{SiO}_{2}$. In vacuo XPS measurements confirmed this hypothesis based on the reaction of $\mathrm{Ru}$ with oxygen from the $\mathrm{SiO}_{2}$, followed by oxygen surface segregation.
\end{abstract}




\subsection{Introduction}

In the last decades, ruthenium thin films have been used in several applications. ${ }^{14}$ $\mathrm{Ru}$ has turned out to be one of the most active catalysts in the ammonia synthesis. ${ }^{330}$ Due to its low resistivity and low solubility with $\mathrm{Cu},{ }^{3} \mathrm{Ru}$ has been used as a $\mathrm{Cu}$ diffusion barrier and/or $\mathrm{Cu}$ seed layer in integrated circuits with copper interconnect technology. ${ }^{4}$ Other applications for Ru thin films are as bottom electrode in capacitors based on high$\mathrm{K}$ materials, ${ }^{6}$ or as capping layer for optics designed for extreme ultraviolet lithography (EUVL) ${ }^{7,8,9,10}$ due to its low sensitivity for oxidation. ${ }^{11}$ In the last three applications, diffusion (either copper or oxygen) towards deeper layers is one of the main threats for their performance. ${ }^{54,322,331}$ In the fabrication of copper-based chips, silicon forms deeplevel traps when it is intermixed with copper. ${ }^{332}$ High-K capacitors are fabricated at high temperatures and under an oxidative environment. In such conditions, the poly-Si plug attached to the electrode is oxidized, resulting in a decrease of the dielectric constant. ${ }^{85}$ The cracking of water vapor present in a vacuum system by EUV light leads to oxidation of the Si/Mo multilayer mirror, causing a reflectance drop. ${ }^{132}$ In all three cases, the underneath Si layer should not be directly in contact with either copper or an oxidative atmosphere. Thus, the Ru protective layer should be a continuous and homogenously closed layer. Like most metals, $\mathrm{Ru}$ forms a polycrystalline structure after a few nanometers which allows $\mathrm{Cu}$ or $\mathrm{O}$ diffusion through grain boundaries. ${ }^{325,333,334}$ For this reason an ultrathin amorphous Ru layer is necessary as an effective diffusion barrier. For instance, a $\mathrm{Ru}$ layer requires a minimum optimized thickness $<5 \mathrm{~nm}$ for a barrier/seed

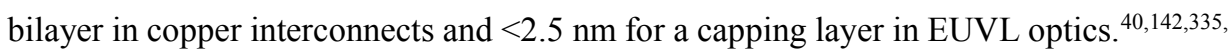

To study the growth of such an ultrathin Ru film, a technique that allows an accurate control of the initial growth stages is needed, including an accurate determination of the thickness where the layer closes. The limited availability of techniques for in situ monitoring thin film growth in the sub- and nanometer scales has turned out to be a problem. ${ }^{274,275}$ Short diffusion lengths involved, necessity of ultra-high vacuum tools, possible matrix effects or variations in morphology and interlayer roughness/intermixing during growth, the need for high-precision real-time measurements and compatibility with amorphous materials are some of the main difficulties to overcome.

Low energy ion scattering (LEIS) is a surface analysis technique that uses the bombardment of noble gas ions $\left(\mathrm{He}^{+}, \mathrm{Ne}^{+}\right.$or $\left.\mathrm{Ar}^{+}\right)$towards the sample (target) with ionenergies between 0.5 and $10 \mathrm{keV}$. Since the scattering cross-sections scale with $1 / E^{2}$, the cross-sections are much larger for LEIS compared to other scattering techniques. A second feature is the effective neutralization of all projectiles that penetrate the sample beyond the first monolayer. As a result, LEIS is highly surface-sensitive and can be used to determine the atomic composition of the outermost surface atomic layer. Detection of sub-surface scattered neutrals is possible due to the re-ionization processes occurring at 
the surface, and an in-depth profile is accessible within the near-surface region (0 to 10 $\mathrm{nm})$. In addition, LEIS can be used on either rough or flat surfaces of either crystalline or amorphous nature. In many cases, LEIS surface quantification is not impeded by matrix effects, although it should be noted that matrix effects are reported for certain projectile/target combinations. ${ }^{270}$ LEIS analyzers have improved in recent years, and a new advanced equipment has been developed, the so-called high-sensitivity low energy ion scattering (HS-LEIS), which requires very low ion fluency for the measurement due to a special detection geometry that enables a "static" analysis with negligible damage by the probing ions, and has resulted in better detection limits for the surface elements. ${ }^{276}$

Very few growth studies are reported for $\mathrm{Ru}$ on $\mathrm{Si}^{183,184}$ To the best of our knowledge, there are no growth studies at room temperature reported which investigate the initial stages of growth of thin Ru films on amorphous Si. In this chapter, we use HSLEIS for in vacuo monitoring $\mathrm{Ru}$ growth on a-Si, $\mathrm{SiN}$ and $\mathrm{SiO}_{2}$ substrate layers. We also show that from $\mathrm{Ru}$ surface coverages, in-depth Ru profiles can be reconstructed for these systems. In order to distinguish different contributions to the intermixing between $\mathrm{Ru}$ and $\mathrm{Si}$ during growth, the reverse system ( $\mathrm{Si}$ on $\mathrm{Ru})$ is also investigated.

\subsection{Experimental}

Samples were prepared with Ru layers from 0 to $5 \mathrm{~nm}$ deposited on top of $5 \mathrm{~nm} \mathrm{Si}$, $1.5 \mathrm{~nm} \mathrm{SiN} / 4 \mathrm{~nm} \mathrm{Si}$ and $1.5 \mathrm{~nm} \mathrm{SiO} / 4 \mathrm{~nm} \mathrm{Si}$, respectively. Additional samples were prepared with Si layers from 0 to $5 \mathrm{~nm}$ deposited onto $5 \mathrm{~nm} \mathrm{Ru}$. All samples were grown onto 25 x $25 \mathrm{~mm}$ natively oxidized super-polished Si substrates and prepared using DC magnetron sputtering at room temperature in an Ultra High Vacuum (UHV) setup with base pressure $<2 \times 10^{-10}$ mbar. (See deposition conditions in Table 5.1, and layered sample models in Fig. 5.1).

Table 5.1. Summary of deposition conditions for the $\mathrm{Ru}, \mathrm{Si}, \mathrm{SiN}$ and $\mathrm{SiO}_{2}$ layers

\begin{tabular}{rcccc}
\hline \hline & $\mathrm{Ru}$ & $\mathrm{Si}$ & $\mathrm{SiN}$ & $\mathrm{SiO}_{2}$ \\
\hline Magnetron & $\mathrm{Ru}$ & $\mathrm{Si}$ & $\mathrm{Si}$ & $\mathrm{Si}$ \\
$\mathrm{Gas}$ & $\mathrm{Ar}$ & $\mathrm{Ar}$ & $40.6 \%$ & $6.4 \%$ \\
& & & $\mathrm{~N}_{2} /\left(\mathrm{N}_{2}+\mathrm{Ar}\right)$ & $\mathrm{O}_{2} /\left(\mathrm{O}_{2}+\mathrm{Ar}\right)$ \\
Total pressure & $1.5 \mathrm{E}-3$ & $1.5 \mathrm{E}-3$ & $1.1 \mathrm{E}-3$ & $7.8 \mathrm{E}-4$ \\
(mbar) & & & & \\
Voltage (V) & $300 \pm 5$ & $400 \pm 5$ & $382 \pm 5$ & $302 \pm 5$ \\
Power (W) & $8 \pm 0.5$ & $8 \pm 0.5$ & $8 \pm 0.5$ & $8 \pm 0.5$ \\
\hline \hline
\end{tabular}


(a)

$\mathrm{X} \mathrm{nm} \mathrm{Ru}$
$5.0 \mathrm{~nm} \mathrm{Si}$
$\mathrm{Si}(100)$
$X=0$ to $5 \mathrm{~nm}$

(b)

$\mathrm{X} \mathrm{nm} \mathrm{Ru}$
$1.5 \mathrm{~nm} \mathrm{SiN}$
$4.0 \mathrm{~nm} \mathrm{Si}$
$\mathrm{Si}(100)$

(c)

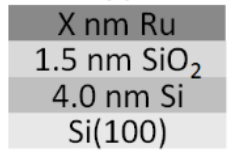

(d)

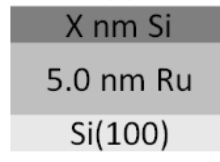

Fig. 5.1. Layered sample models for $\mathrm{Ru}$ on (a) $\mathrm{Si}$, (b) $\mathrm{SiN}$ and (c) $\mathrm{SiO}_{2}$, and (d) $\mathrm{Si}$ on $\mathrm{Ru}$.

All layer thicknesses were monitored using quartz mass balances (QMB), calibrated by ex situ X-ray reflectivity (XRR) using a PANalytical Empyrean X-ray diffractometer $(\mathrm{Cu}-\mathrm{K} \alpha$ radiation, $0.154 \mathrm{~nm})$. Surface morphologies were analyzed using an ex situ BRUKER Dimension Edge atomic force microscope (AFM) equipped with a $\mu$ masch Hi'Res-C14 tip.

In vacuo X-ray photoelectron spectroscopy (XPS) measurements were performed for studying the initial stages of $\mathrm{Ru}$ growth on $\mathrm{SiO}_{2}$, and to determine the stoichiometry of, and the optimal gas mixture for the deposited $\mathrm{SiO}_{\mathrm{x}}$ and $\mathrm{SiN}_{\mathrm{x}}$ layers (see conditions in Table 5.1). The instrument used was a Thermo Theta Probe spectrometer equipped with a monochromatic Al-K $\alpha$ radiation $(\mathrm{h} v=1486.6 \mathrm{eV})$. The optimal $\mathrm{O} / \mathrm{Si}$ ratio obtained for the $\mathrm{SiO}_{\mathrm{x}}$ layers was about 2.0, near to stoichiometric $\mathrm{SiO}_{2} \cdot{ }^{38}$ However, the optimal N/Si ratio obtained for the $\mathrm{SiN}_{\mathrm{x}}$ layers was close to 1.0 , which means that the produced $\mathrm{SiN}_{\mathrm{x}}$ layers were sub-stoichiometric and silicon-rich, when compared to bulk $\mathrm{Si}_{3} \mathrm{~N}_{4} \cdot{ }^{38}$ Several studies also showed this behavior for room-temperature deposition of $\mathrm{SiN}_{\mathrm{x}}$ by reactive magnetron sputtering in $\mathrm{Ar}+\mathrm{N}_{2}$ mixture. ${ }^{336,337}$ For simplicity this sub-stoichiometric $\mathrm{SiN}_{\mathrm{x}}$ layer is referred in the text as $\mathrm{SiN}$.

In vacuo and "in-real-time" HS-LEIS measurements during Ru and Si growth were carried out using an ION-TOF GmbH Qtac ${ }^{100}$ HS-LEIS spectrometer with a base pressure of $2 \times 10^{-10} \mathrm{mbar}$. This setup has a fixed scattering geometry, where the primary ions are directed perpendicular towards the sample surface, and only the scattered ions (no neutrals) are detected at a fixed scattering angle of $145^{\circ}$ by a double toroidal electrostatic analyzer with full azimuthal acceptance. This, in combination with a channel plate detector for simultaneous energy detection, provides a higher sensitivity than conventional LEIS analyzers, and enables a non-destructive ("static") analysis. A more detailed description of the setup can be found in ref. ${ }^{276}$.

Deposited samples were transferred under vacuum $\left(2 \times 10^{-10} \mathrm{mbar}\right)$ to the LEIS analysis chamber. The work pressure in the LEIS analysis chamber was $\sim 2 \times 10^{-8}$ mbar during all measurements, due to $\mathrm{He}$ used in the ion gun. Sample transfer and measurement were carried out within $<10$ min after deposition in order to avoid surface contamination. All LEIS measurements were performed using a $\mathrm{He}^{+}$ion beam with an energy of $3 \mathrm{keV}$ and with a current of 4-6 nA, measured with a Faraday cup. The average ion dose was $1.5 \times 10^{14} \mathrm{He}^{+}$ions $/ \mathrm{cm}^{2}$, below the "static limit" for $3 \mathrm{keV} \mathrm{He}$ ions which 
implies that less than $1 \%$ of the surface is sputtered away during the LEIS analysis. ${ }^{279,338,339}$

\subsection{Results}

The results are presented in three sections. First, we start with the determination of the surface coverages and the thickness of the closed layer for Ru grown on $\mathrm{Si}, \mathrm{SiN}$ and $\mathrm{SiO}_{2}$ layers. Second, we reconstruct the in-depth $\mathrm{Ru}$ profiles from the $\mathrm{Ru}$ surface coverages. And third, we perform the same study for Si grown on Ru.

\subsubsection{Surface coverages and closed layer determination for $R u$ on $\mathrm{Si}, \mathrm{SiN}$ and $\mathrm{SiO}_{2}$}

A typical LEIS spectrum for $\mathrm{He}^{+}$ions gives two main kinds of information: the elemental composition of the outermost atomic layer and the in-depth concentration of atoms just below the surface (0 to $10 \mathrm{~nm}$ ). The composition of this outermost layer is defined by the surface peaks of the elements present on the surface, and the sub-surface concentration of these elements is determined by their "low-energy tails" to the peaks. ${ }^{270}$ Examples of LEIS spectra for $3 \mathrm{keV} \mathrm{He}$ ions scattered from $0.7 \mathrm{~nm}$ and $4.7 \mathrm{~nm} \mathrm{Ru}$ samples grown on $5.0 \mathrm{~nm} \mathrm{Si}$ are depicted in Fig. 5.2 (black and red solid lines, respectively).

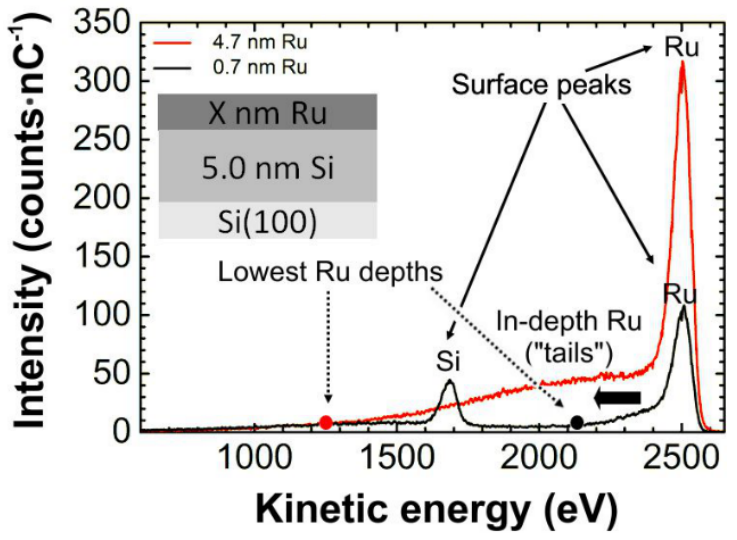

Fig. 5.2. LEIS spectra of $3 \mathrm{keV} \mathrm{He}{ }^{+}$scattered from $0.7 \mathrm{~nm}$ (black solid line) and $4.7 \mathrm{~nm}$ (red solid line) Ru samples grown on $5.0 \mathrm{~nm}$ Si on Si(100) substrate. Ru and Si surface peaks, lowest Ru depths and in-depth $\mathrm{Ru}$ "tails" are pointed out.

We define a layer of one or more elements to become closed when its surface only contains these elements and other elements from the underneath substrate layer are no longer present on this surface. Since our LEIS setup has a high sensitive to the elements on the surface, a layer will be considered a "closed layer" when the surface peaks of the elements of the substrate layer vanish and when the surface peak of the element of the 
growing layer saturates and does not grow further. In the example spectra presented in Fig. 5.2., the $0.7 \mathrm{~nm} \mathrm{Ru}$ layer (black solid line) is an "open layer", since there is a clear $\mathrm{Si}$ surface peak and the Ru peak is not yet saturated. In contrast, the $4.7 \mathrm{~nm} \mathrm{Ru}$ layer (red solid line) is a "closed layer". No Si surface peak is detected and the Ru surface peak is saturated.

Fig. 5.3 presents LEIS spectra of the deposited layers on $\mathrm{Si}$ (a), $\mathrm{SiN}$ (b) and $\mathrm{SiO}_{2}$ (c). For Ru grown on $\mathrm{Si}$, the $\mathrm{Si}$ surface peak decreases and the Ru surface peak increases during growth (Fig. 5.3(a)). The Ru surface peak is saturated at about $4.7 \mathrm{~nm} \mathrm{Ru}$ and there is no signal from $\mathrm{Si}$ at the surface for that thickness. This means that about $4.7 \mathrm{~nm}$ $\mathrm{Ru}$ are needed to close the $\mathrm{Ru}$ layer when growing on top of $\mathrm{Si}$. This large amount of material to close the layer might be related to a lot of intermixing by $\mathrm{RuSi}_{\mathrm{x}}$ formation. For $\mathrm{Ru}$ grown on $\mathrm{SiN}$, the Si peak fully disappears at about $3.2 \mathrm{~nm}$ when the Ru peak saturates (Fig. 5.3(b)). Thus, $3.2 \mathrm{~nm} \mathrm{Ru}$ are enough to close the Ru layer grown on SiN. Compared to the previous case where $\mathrm{Ru}$ is grown on $\mathrm{Si}$, the layer closes earlier by nitrogen passivation of the $\mathrm{Si}$ surface, and the $\mathrm{RuSi}_{\mathrm{x}}$ formation is reduced. For $\mathrm{Ru}$ grown on $\mathrm{SiO}_{2}$, the Si peak disappears at about $2.0 \mathrm{~nm} \mathrm{Ru}$, coinciding with the saturation of the $\mathrm{Ru}$ surface peak (Fig. 5.3(c)). As a result, only $2.0 \mathrm{~nm} \mathrm{Ru}$ are needed to close the $\mathrm{Ru}$ layer on $\mathrm{SiO}_{2}$, which indicates that oxygen passivates $\mathrm{Si}$ more strongly than nitrogen. A comparable LEIS study performed by Shin et al. ${ }^{140}$ for $\mathrm{Ru}$ grown on $\mathrm{SiO}_{2}$ observed the $\mathrm{Ru}$ layer to become closed after a thickness near $2.2 \mathrm{~nm}$. This value is similar to the 2.0 $\mathrm{nm}$ value obtained by our high-sensitivity LEIS study on this system.

It should be noted here that LEIS cannot distinguish between intermixing and 3D island growth. The formation of 3D islands would give similar LEIS signals as intermixing by silicide formation during $\mathrm{Ru}$ growth, which might lead to misinterpretation of our results. Therefore, surface morphologies of samples for several $\mathrm{Ru}$ thicknesses on all three substrate layers were studied by AFM. As an example, samples with an intermediate $\mathrm{Ru}$ thickness of $\sim 1.3 \mathrm{~nm}$ exhibit a root mean square (RMS) roughness of $0.22 \pm 0.05,0.23 \pm 0.05$ and $0.21 \pm 0.05 \mathrm{~nm}$ for $\mathrm{Ru}$ grown on $\mathrm{Si}, \mathrm{SiN}$ and $\mathrm{SiO}_{2}$, respectively (see AFM images of these samples in Figs. 5.4(a), 5.4(b), and 5.4(c)). These values and the values obtained for all other thicknesses are comparable and very close to the uncoated $\mathrm{Si}(100)$ substrate, which presents an average RMS roughness of $0.20 \pm 0.05$ $\mathrm{nm}$. Thus, we can attribute the relatively large amount of Ru needed for forming a closed layer to intermixing, and not to $3 \mathrm{D}$ growth.

$\mathrm{Ru}, \mathrm{Si}, \mathrm{N}$ and $\mathrm{O}$ surface peaks in Fig. 5.3 originate from $\mathrm{He}^{+}$ions that are backscattered in a single elastic binary collision from a $\mathrm{Ru}, \mathrm{Si}, \mathrm{N}$, or $\mathrm{O}$ atom at the surface of the sample. The intensity of each surface peak is proportional to the surface concentration (or coverage) of the corresponding element. ${ }^{276}$ Both peak height and area 
can be used for quantitative surface composition analysis. In the following analysis we use peak area, which provides more accurate results, as described in ref. ${ }^{270}$.

(a)

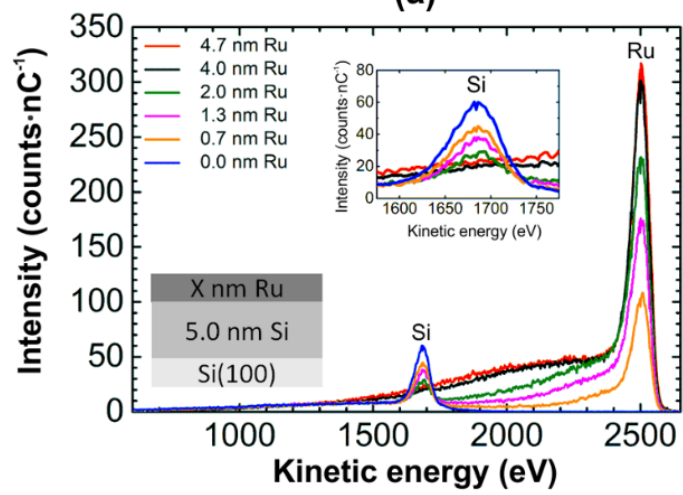

(b)

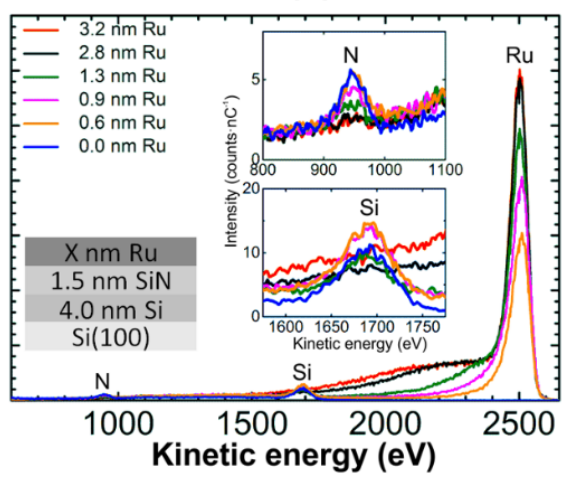

(c)

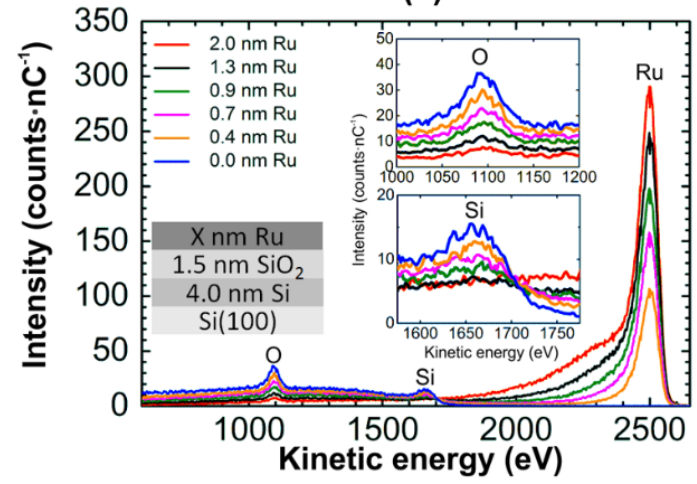

Fig. 5.3. LEIS spectra of (a) 0.0 to $4.7 \mathrm{~nm}$ Ru on $5.0 \mathrm{~nm}$ Si samples, (b) 0.0 to $3.2 \mathrm{~nm}$ Ru on $1.5 \mathrm{~nm}$ $\mathrm{SiN} / 4.0 \mathrm{~nm}$ Si samples, and (c) 0.0 to $2.0 \mathrm{~nm} \mathrm{Ru}$ on $1.5 \mathrm{~nm} \mathrm{SiO} / 4.0 \mathrm{~nm} \mathrm{Si}$ samples onto $\mathrm{Si}(100)$ substrates. Si peak zoom in (a), N and Si peaks zoom in (b), and O and Si peaks zoom in (c). Leftbottom insets in (a), (b) and (c) show the layered models of the deposited structures.

(a) $\mathrm{Ru}$ on $\mathrm{Si}$

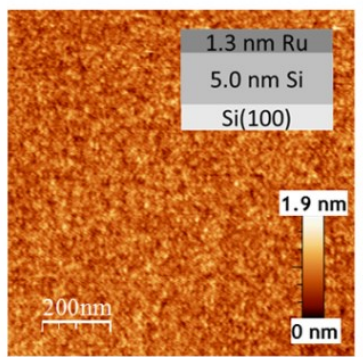

(b) Ru on SiN

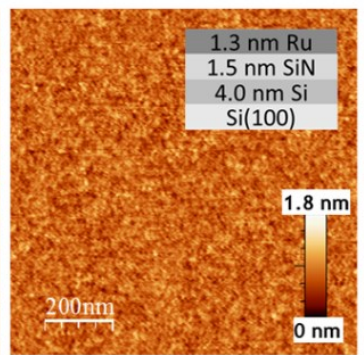

(c) Ru on $\mathrm{SiO}_{2}$

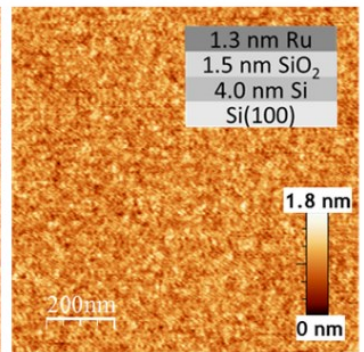

Fig. 5.4. AFM images $(1 \mathrm{x} 1 \mu \mathrm{m})$ of samples with intermediate thickness of $\mathrm{Ru}$ (no closed layer) on $\mathrm{Si}$ (a), $\mathrm{SiN}$ (b), and $\mathrm{SiO}_{2}$ (c). Layered models of the deposited structures are shown in insets. 
Fig. 5.5(a) shows the surface peak area from Ru (squares, left axis) and Si (triangles, right axis) for $\mathrm{Ru}$ grown on $\mathrm{Si}$. If we plot these surface peak areas for $\mathrm{Ru}$ against $\mathrm{Si}$, they follow a clear linear trend (Fig. 5.5(b)). From the extrapolation of this line to the ordinate and the coordinate axis, the reference peak areas (in case of full coverage) for Ru and $\mathrm{Si}$ can be estimated. This line implicitly assumes that for a binary system such as $\mathrm{Ru}$ on $\mathrm{Si}$ where there are no other elements, the sum of their surface coverages is equal to 1 (a full monolayer coverage). This assumption is analogous to the Vegard's law for the volume of an alloy applied to the surface and is only valid in the absence of matrix effects, which means that the sensitivity for a given element does not depend on its neighboring atoms. $^{270,340}$
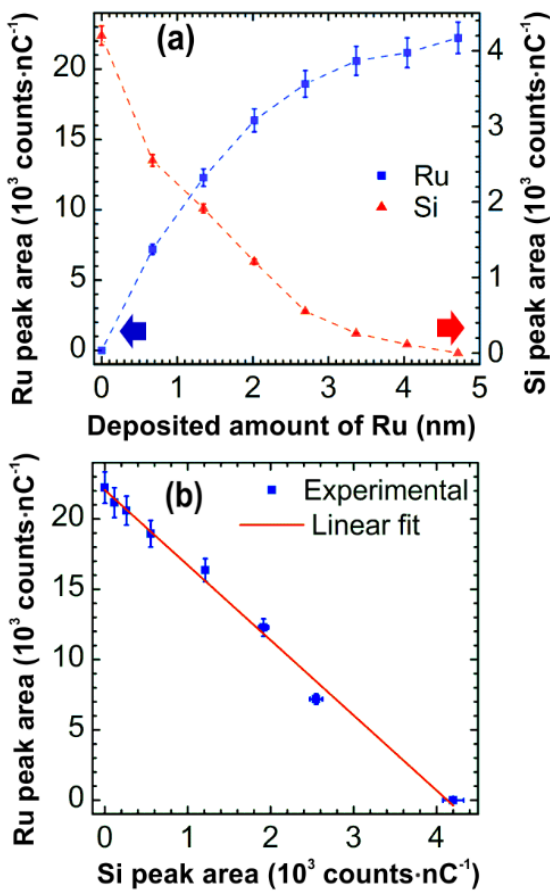

Fig. 5.5. (a) Ru (squares) and $\mathrm{Si}$ (triangles) surface peak areas for Ru grown on $\mathrm{Si}$. The dashed lines are guides for the eye. (b) Ru surface peak area vs. Si surface peak area for the data shown in (a). The solid line is a linear fit to the data. This linear relationship shows the absence of matrix effects and that Vegard's law holds for the surface..$^{270,340}$

The determined reference peak area values for $\mathrm{Ru}$ and $\mathrm{Si}$ samples are $(2.2 \pm 0.1) \mathrm{X}$ $10^{4}$ and $(4.1 \pm 0.1) \times 10^{3}$ counts $\cdot \mathrm{nC}^{-1}$, respectively. The surface atomic density $N_{i}^{\text {ref }}$ of an element $i$ in a reference sample (compound or element) with known mass density $\rho$, can be estimated by

$$
N_{i}^{r e f} \approx\left(\frac{n_{i} \rho N_{A V}}{M}\right)^{2 / 3},
$$


where $n_{i}$ is the stoichiometric number of the element $i$ in the compound ( $n_{i}=1$ for a single element), $M$ is the molar mass of the compound (or element), and $N_{A V}$ is Avogadro's number. ${ }^{341}$ The surface atomic densities $N_{i}^{r e f}$ for $\mathrm{Ru}, \mathrm{Si}, \mathrm{N}$ and $\mathrm{O}$ atoms are calculated by Eq. (5.1), using mass densities of $12.2 \pm 0.3,2.3 \pm 0.3,3.6 \pm 0.3$ and $2.4 \pm 0.3 \mathrm{~g} \cdot \mathrm{cm}^{-3}$, obtained from the respective fits to the XRR data of $10 \mathrm{~nm}$-thick $\mathrm{Ru}, \mathrm{Si}, \mathrm{SiN}$ and $\mathrm{SiO}_{2}$ deposited samples.

Knowing the surface peak areas $S_{j}$, the reference peak areas $S_{j}^{r e f}$ and the surface atomic densities $N_{j}^{r e f}$ for the different surface elements (from $j=1$ to $j_{\max }$ ), one can determine the surface coverage $C_{i}^{\text {surf }}$ for an element $i$, expressed as surface atomic fraction by ${ }^{270}$

$$
C_{i}^{\text {surf }}=\left[\sum_{j=1}^{j_{\max }} \frac{S_{j} S_{i}^{r e f} N_{j}^{r e f}}{S_{i} S_{j}^{r e f} N_{i}^{r e f}}\right]^{-1} .
$$

Surface coverages for $\mathrm{Ru}, \mathrm{Si}, \mathrm{N}$ and $\mathrm{O}$ elements for $\mathrm{Ru}$ layers on $\mathrm{Si}, \mathrm{SiN}$ and $\mathrm{SiO}_{2}$ are determined by Eq. (5.2) using the obtained surface peak areas. $\mathrm{N}$ and $\mathrm{O}$ reference peak areas are numerically estimated as follows, since pure reference surfaces for $\mathrm{N}$ and $\mathrm{O}$ do not exist. For each Ru deposited amount, we know that the surface Vegard's law must be fulfilled ${ }^{340}$

$$
\sum_{j=1}^{j_{\max }} C_{j}^{\text {surf }}=1
$$

We assume a certain value for the $\mathrm{N}$ or $\mathrm{O}$ reference peak area and then we use this initial value to calculate all surface coverages for $\mathrm{Ru}$ grown either on $\mathrm{SiN}$ or on $\mathrm{SiO}_{2}$. Then, this value is recursively changed till the Vegard's condition is valid for all deposited thicknesses. The obtained reference peak areas for $\mathrm{N}$ and $\mathrm{O}$ are $345 \pm 24$ and $1179 \pm 83$ counts $\cdot \mathrm{nC}^{-1}$, respectively. To ensure the validity of this procedure for the $\mathrm{O}$ case, a 10 $\mathrm{nm} \mathrm{Si}$ layer is deposited onto a $\mathrm{Si}(100)$ substrate and in vacuo transferred to the LEIS main chamber. Consecutive $3 \mathrm{keV} \mathrm{He}{ }^{+}$LEIS measurements are performed at room temperature after different amounts of $\mathrm{O}_{2}$ (several hundred seconds at $\sim 1.5 \times 10^{-7}$ mbar $\mathrm{O}_{2}$ ) till $\mathrm{O}$ saturation. Vegard's law is also fulfilled for this system and no matrix effects are detected. By linear extrapolation with the abscissa axis when plotting the surface peak areas of $\mathrm{Si}$ against $\mathrm{O}$, the reference value for $\mathrm{O}$ can be extracted. The experimentally determined $\mathrm{O}$ reference area is $1180 \pm 83$ counts $\cdot \mathrm{nC}^{-1}$, which agrees well with the previously calculated value.

Fig. 5.6 presents the normalized peak areas and the surface coverages for $\mathrm{Ru}$ (squares), Si (triangles), $\mathrm{N}$ and $\mathrm{O}$ (circles) elements for Ru on $\mathrm{Si}$ (a, d), $\mathrm{SiN}$ (b, e) and $\mathrm{SiO}_{2}(\mathrm{c}, \mathrm{f})$ layers. 

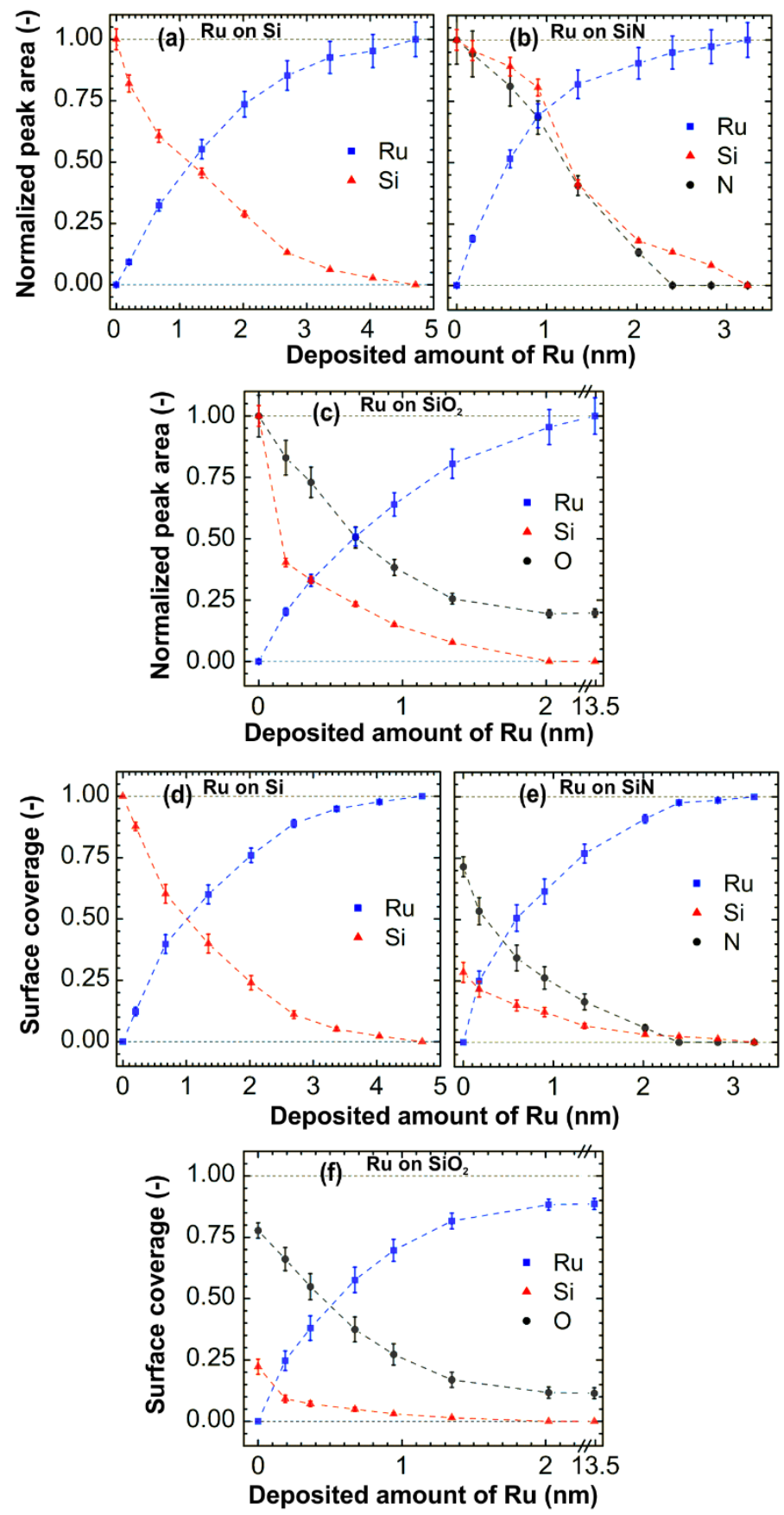

Fig. 5.6. Normalized peak areas and surface coverages for $\mathrm{Ru}$ (squares), $\mathrm{Si}$ (triangles), $\mathrm{N}$ and $\mathrm{O}$ (circles) elements for $\mathrm{Ru}$ on $\mathrm{Si}$ (a, d), $\mathrm{SiN}$ (b, e) and $\mathrm{SiO}_{2}$ (c, f) layers. The dashed lines through the data points are guides for the eye. Horizontal dashed lines in each graph represent the limits (from 0 to 1) for the normalized peak area and the surface coverage. 
There is not much difference between the normalized peak area and the surface coverage for Ru grown on Si (Figs. 5.6(a) and 5.6(d)), since it is a simple binary system and the correction factors introduced by Eq. (5.1) are not very significant. $\mathrm{N}$ and Si peak areas decrease similarly while $\mathrm{Ru}$ grows for $\mathrm{Ru}$ on $\mathrm{SiN}$ system, but the $\mathrm{N}$ signal vanishes at about $2.4 \mathrm{~nm} \mathrm{Ru}$ instead of $3.2 \mathrm{~nm} \mathrm{Ru}$ for the Si signal (Fig. 5.6(b)). This is due to a lower elemental sensitivity factor for $\mathrm{N}$ compared to $\mathrm{Si}^{270}$ Likewise, there is a similar decrease of $\mathrm{O}$ and $\mathrm{Si}$ peak areas while the $\mathrm{Ru}$ peak area increases for $\mathrm{Ru}$ grown on $\mathrm{SiO}_{2}$ (Fig. 5.6(c)). Although the $\mathrm{Si}$ signal vanishes at $2.0 \mathrm{~nm} \mathrm{Ru}$, the $\mathrm{O}$ signal is still present even for the $13.5 \mathrm{~nm} \mathrm{Ru}$ sample. There is an almost constant $\mathrm{O}$ coverage of about $12 \%$ for the different closed layer thicknesses $(\geq 2.0 \mathrm{~nm} \mathrm{Ru})$ as depicted by circles in Fig. 5.6(f). This might be caused by surface contamination during sample manipulation or due to the fact that part of the oxygen is diffusing up from the $\mathrm{SiO}_{2}$ film through the $\mathrm{Ru}$ layer and reacting with the $\mathrm{Ru}$ surface ( $\mathrm{O}$ surface segregation). Contamination during manipulation may be discarded since in both $\mathrm{Si}$ and $\mathrm{SiN}$ growth studies, there is no apparent detected surface $\mathrm{O}$ signal (see Figs. 5.3(a) and 5.3(b), respectively).

To investigate oxygen segregation during $\mathrm{Ru}$ growth and oxygen reaction with $\mathrm{Ru}$, in vacuo XPS measurements are performed on several deposited $\mathrm{Ru}$ samples with thicknesses ranging from 0.0 to $5.5 \mathrm{~nm}$ on top of $1.5 \mathrm{~nm} \mathrm{SiO}_{2} / 4.0 \mathrm{~nm}$ Si bilayers (see Si$2 \mathrm{p}$ and O-1s XPS spectra in Figs. 5.7(a) and 5.7(b), respectively).

(a) Si-2p

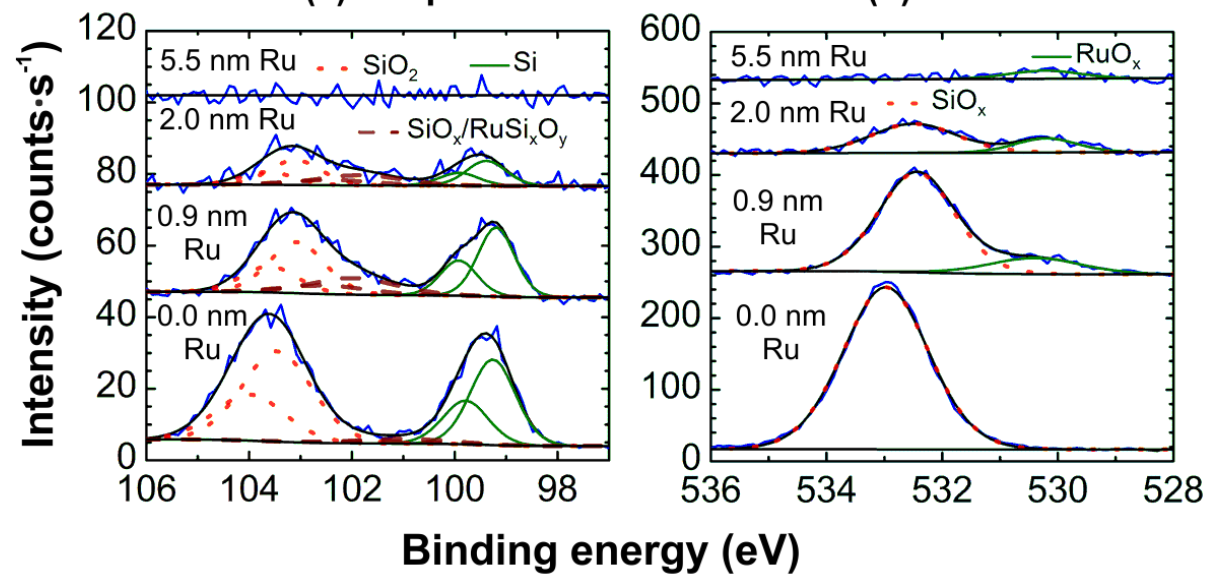

Fig. 5.7. $\mathrm{Si}-2 \mathrm{p}$ (a) and $\mathrm{O}-1 \mathrm{~s}$ (b) XPS spectra for $\mathrm{Ru}$ deposited on $\mathrm{SiO}_{2}$. Si-2 $\mathrm{p}_{1 / 2}$ (left) and $\mathrm{Si}-2 \mathrm{p}_{3 / 2}$ (right) peaks which correspond to elemental $\mathrm{Si}$ (green solid lines), $\mathrm{SiO}_{\mathrm{x}}(1 \leq \mathrm{x}<2) / \mathrm{RuSi}_{\mathrm{x}} \mathrm{O}_{\mathrm{y}}$ (brown dashed lines), and $\mathrm{SiO}_{2}$ (red dot lines) are displayed in (a). O-1s peaks for $\mathrm{SiO}_{\mathrm{x}}$ (red dashed lines) and $\mathrm{RuO}_{\mathrm{x}}$ (green solid lines) are depicted in (b). Note that all spectra are shifted in the intensity axis in order to visualize all peaks. 
For $0 \mathrm{~nm} \mathrm{Ru}$, there are only $\mathrm{SiO}_{2}$ and $\mathrm{Si}$ peaks in the case of the $\mathrm{Si}-2 \mathrm{p}$ peak, as well as a very small amount of sub-stoichiometric $\mathrm{SiO}_{\mathrm{x}}(1 \leq \mathrm{x}<2)$ on the interface between $\mathrm{Si}$ and $\mathrm{SiO}_{2}$ (see Fig. 5.7(a)). For the O-1s peak, only one peak corresponding to $\mathrm{SiO}_{\mathrm{x}}$ is observed (see Fig. 5.7(b)). While $\mathrm{Ru}$ grows, there is a clear peak of $\mathrm{RuO}_{\mathrm{x}}$, already appearing at $0.9 \mathrm{~nm} \mathrm{Ru}$ for the $\mathrm{O}-1 \mathrm{~s}$ peak and the formation of more sub-stoichiometric $\mathrm{SiO}_{\mathrm{x}}$ or $\mathrm{RuSi}_{\mathrm{x}} \mathrm{O}_{\mathrm{y}}$ for the $\mathrm{Si}-2 \mathrm{p}$ peak as depicted in Figs. 5.7(b) and 5.7(a), respectively. This shows that part of the oxygen from the $\mathrm{SiO}_{2}$ layer is reacting with the $\mathrm{Ru}$ layer, even though formation of $\mathrm{SiO}_{\mathrm{x}}$ is thermodynamically more favorable. ${ }^{38}$ As we increase the $\mathrm{Ru}$ thickness, the Si-2p peaks decrease until they disappear at about $5.5 \mathrm{~nm} \mathrm{Ru}$ (Fig. 5.7(a)). In the case of the O-1s peak, the $\mathrm{SiO}_{x}$ peak has vanished at $5.5 \mathrm{~nm} \mathrm{Ru}$ but still there is a $\mathrm{RuO}_{x}$ peak (Fig. 5.7(b)). This confirms our hypothesis based on reaction of $\mathrm{Ru}$ with $\mathrm{O}$ at the $\mathrm{Ru}-\mathrm{SiO}_{2}$ interface, followed by oxygen surface segregation for increasing $\mathrm{Ru}$ thickness, forming surface $\mathrm{RuO}_{\mathrm{x}}$. This oxygen surface segregation effect might be due to the fact that the $\mathrm{Ru}$ surface strongly reacts with oxygen. Although $\mathrm{Ru}$ is a lowoxidation metal, ${ }^{11}$ oxygen prefers to be adsorbed on its surface, rather than remain in the bulk of the layer. ${ }^{14}$ Thus, a small amount of oxygen from the $\mathrm{Ru}-\mathrm{SiO}_{2}$ interface may diffuse up towards the Ru surface.

For comparing the $\mathrm{Ru}$ growth on $\mathrm{Si}, \mathrm{SiN}$ and $\mathrm{SiO}_{2}$, the previously determined surface coverages for the Ru-on- $\mathrm{SiO}_{2}$ system (shown in Fig. 5.6(f)) are then modified, compensating for the fact that $100 \% \mathrm{Ru}$ coverage is not reached due to $\mathrm{O}$ surface segregation. Si surface coverage remains unaltered since this segregation effect only affects $\mathrm{Ru}$ and $\mathrm{O}$ coverages. To correct both $\mathrm{Ru}$ and $\mathrm{O}$ coverages for this phenomenon, the part of the total $\mathrm{O}$ coverage corresponding to the oxygen segregated towards the $\mathrm{Ru}$ surface $\left(\mathrm{O}^{\text {segr }}\right.$ coverage $)$ is determined for every deposited $\mathrm{Ru}$ thickness, under the assumption that the ratio $\mathrm{O}^{\text {segr }} / \mathrm{Ru}$ surface coverage is the same for each thickness, using the value for $2 \mathrm{~nm}$ or more deposited $\mathrm{Ru}$ as reference. By subtracting these $\mathrm{O}^{\text {segr }}$ values (see Fig. 5.8) from the total O coverage, a "corrected O coverage" is obtained, which corresponds only to the $\mathrm{O}$ bonded to the $\mathrm{Si}$. By correcting the $\mathrm{Ru}$ coverage for the segregated $\mathrm{O}$, the comparison of the three model systems becomes more straightforward, as depicted in Fig. 5.9.

As previously discussed, the Ru layer closes sooner when increasing Si passivation (see vertical dashed lines in Fig. 5.9). One possible explanation to this behavior could be attributed to thermodynamics and specifically when comparing the enthalpies of formation $\Delta_{\mathrm{f}} H^{\circ}$ of $\mathrm{RuSi}, \mathrm{Si}_{3} \mathrm{~N}_{4}$ and $\mathrm{SiO}_{2}$ (see Table 5.2). $\mathrm{SiO}_{2}$ formation is thermodynamically more favorable than $\mathrm{Si}_{3} \mathrm{~N}_{4}$ formation, and this is followed by $\mathrm{RuSi}$ formation. Since all three reactions of formation are exothermic, more negative values denote more compound stability. For $\mathrm{Ru}$ on $\mathrm{SiN}$, the $\mathrm{Si}-\mathrm{N}$ reaction would be more favorable than the $\mathrm{Ru}-\mathrm{Si}$ reaction, and for $\mathrm{Ru}$ on $\mathrm{SiO}_{2}$, the $\mathrm{Si}-\mathrm{O}$ reaction would be even more favorable than the $\mathrm{Ru}-\mathrm{Si}$ reaction. 
Table 5.2. Standard molar enthalpies of formation $\Delta_{\mathrm{f}} H^{\circ}$ at $25^{\circ} \mathrm{C}$ in $\mathrm{kJ} \cdot \mathrm{mol}^{-1}$ for $\mathrm{RuSi}, \mathrm{Si}_{3} \mathrm{~N}_{4}$ and $\mathrm{SiO}_{2}$.

\begin{tabular}{ccc}
\hline \hline Compound & $\Delta_{\mathrm{f}} H^{\circ}$ & Ref. \\
\hline $\mathrm{RuSi}$ (crys.) & -32.4 & 318 \\
$\mathrm{Si}_{3} \mathrm{~N}_{4}$ (crys.) & -743.5 & 38 \\
$\mathrm{SiO}_{2}$ ( $\alpha$-quarz) & -910.7 & 38 \\
\hline \hline
\end{tabular}

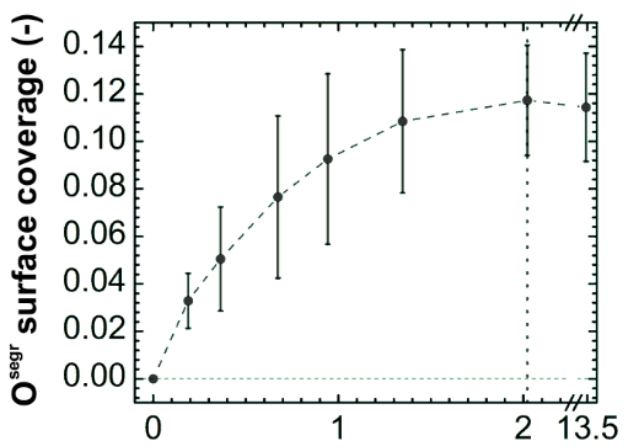

Deposited amount of $\mathrm{Ru}(\mathrm{nm})$

Fig. 5.8. $\mathrm{O}^{\text {seg }}$ surface coverages for $\mathrm{Ru}$ grown on $\mathrm{SiO}_{2}$. The dashed line through the data points is a guide for the eye. The horizontal dashed lines represent the limit for the coverage equal to 0 . The vertical dashed line denotes the $\mathrm{Ru}$ "closed layer" thickness on $\mathrm{SiO}_{2}$.

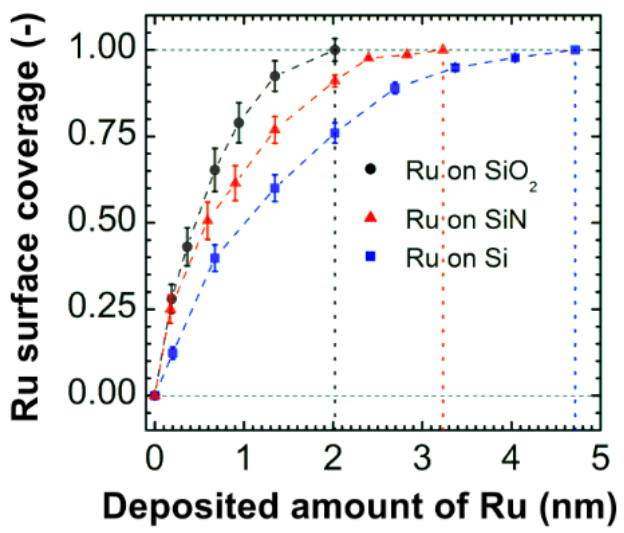

Fig. 5.9. $\mathrm{Ru}$ surface coverages for $\mathrm{Ru}$ grown on $\mathrm{SiO}_{2}$ (circles), $\mathrm{SiN}$ (triangles) and $\mathrm{Si}$ (squares). The dashed lines through the data points are guides for the eye. The two horizontal dashed lines represent the limits for the $\mathrm{Ru}$ surface coverage (from 0 to 1 ). The three vertical dashed lines denote the $\mathrm{Ru}$ “closed layer" thickness on $\mathrm{SiO}_{2}$ (black), $\mathrm{SiN}$ (red) and $\mathrm{Si}$ (blue) layers. 


\subsubsection{In-depth $R u$ profiles from $R u$ surface coverages}

In Sec. 5.3.1, we have used the area of the LEIS surface peaks to determine the surface coverages (or surface concentration). In this section, we propose a method to use these surface coverages to reconstruct the sub-surface concentration. With this approach, we would like to check if the surface LEIS signals, as a function of thickness, can be applied for extracting both surface and in-depth concentration profiles. The procedure for the reconstruction of the in-depth $\mathrm{Ru}$ profiles from the $\mathrm{Ru}$ surface coverages is described as follows.

First, an initial depth profile is proposed under the assumption that $\mathrm{Ru}-\mathrm{Si}$ intermixing is only occurring during $\mathrm{Ru}$ deposition and no further intermixing will take place after deposition at room temperature. Thus, we can assume that the in-depth concentration is equal to the surface concentration (or coverage) after deposition $\left(C=C^{\text {surf }}\right)$. As a first approximation, we propose an error function like model to describe our initial interfacial profile. This approximation can be made by a simple mathematical analysis when considering a Gaussian distribution of the surface heights at the interface. ${ }^{342}$ This approach has already been followed by Névot and Croce, ${ }^{296}$ et al., and mathematically analyzed by Vidal and Vincent, ${ }^{343}$ et al., to describe the interfacial roughness in the XRR analysis of thin films. Our initial proposed profile describes the concentration $C$ of the growing element at a certain depth $z$ from the surface as

$$
C(z)=\frac{1}{2}\left[1+\operatorname{erf}\left(\frac{z_{0}-z}{\sigma \sqrt{2}}\right)\right],
$$

where $z_{0}$ is the average interface depth and $\sigma$ is the characteristic profile width.

From the initially proposed depth profile $C(z)$, one can calculate the deposited thickness $t$ at each depth $z$ by integrating $C(z)$ as

$$
t(z)=\int_{z_{\max }}^{z} C(z) d z,
$$

where $z_{\max }$ is the maximum profile depth which corresponds to a concentration $C\left(z_{\max }\right)=0$. Combining Eq. (5.5) with Eq. (5.4), we can obtain the surface concentration $C$ for each calculated deposited thickness $t$ assuming a certain $\sigma$ value. From the fit of this obtained surface concentration $C(t)$ to the experimental surface coverage $C^{\text {surf }}$ of the growing element for each deposited thickness, and changing the unique free parameter $\sigma$, the indepth profiles can be reconstructed. The $\sigma$ parameter is changed iteratively until the simulated and the experimental data match by a least-squares minimization procedure. ${ }^{328}$ Note that the mean squared error (MSE) from the least-squares procedure is used as a stopping criterion for the iterative process. A MSE value $<0.02$ is applied in all data fits. 
Fig. 5.10(a) shows the in-depth Ru concentration profiles $C(z)$ (Eq. (5.4)) for $\mathrm{Ru}$ grown on $\mathrm{SiO}_{2}$ (black solid line), $\mathrm{SiN}$ (red solid line) and $\mathrm{Si}$ (blue solid line) reconstructed from the fits of the simulated $\mathrm{Ru}$ surface concentration $C(t)$ to the experimental $\mathrm{Ru}$ surface coverage $C^{\text {surf }}$ represented in Fig. 5.10(b) by solid lines and closed symbols, respectively.
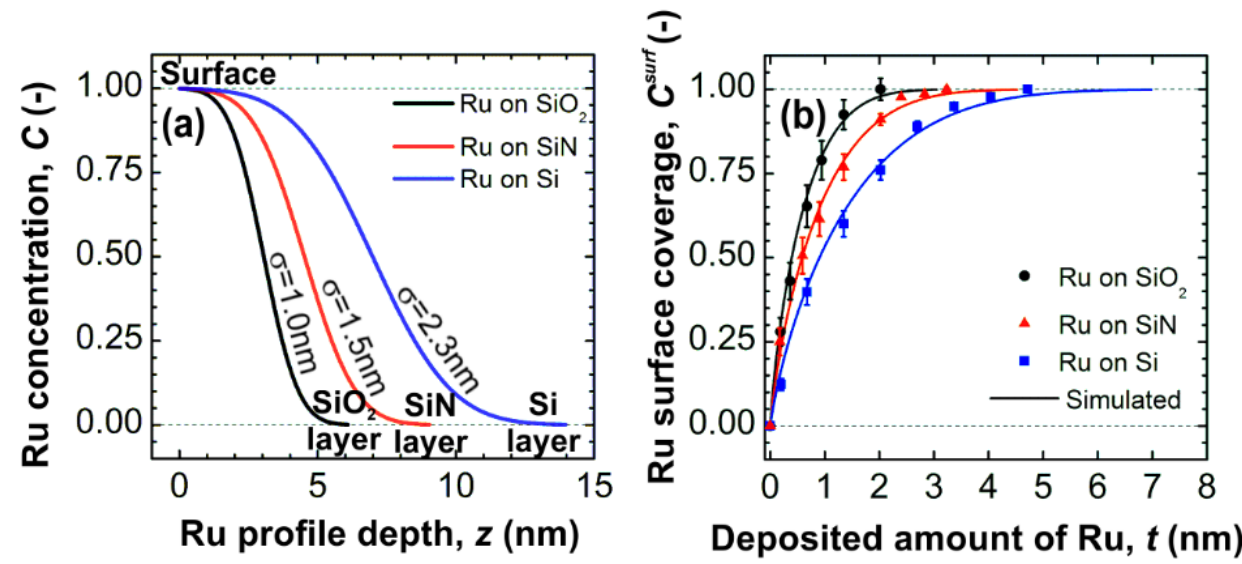

Fig. 5.10. (a) In-depth Ru concentration profiles $C(z)$ described by Eq. (5.4), and characteristic profile widths $\sigma$, for $\mathrm{Ru}$ grown on $\mathrm{SiO}_{2}, \mathrm{SiN}$ and $\mathrm{Si}$. (b) $\mathrm{Ru}$ concentration (surface coverage or in-depth concentration, since we assume $C=C^{\text {surf }}$ ) plotted against experimental (closed symbols) and simulated (solid lines) deposited Ru thickness $t$.

There is a good agreement between experimental surface coverage $C^{\text {surf }}$ and simulated concentration $C(t)$ for each deposited Ru thickness $t$. This means that a simple error function model seems a valid assumption to describe our interfaces. Additional interface profile functions (e.g. linear, double error function) were evaluated but showed less good agreement with the experimental data.

There is still a remaining question to answer about the shape of the in-depth $\mathrm{Ru}$ profiles. Is the shape of the "real" interface corresponding to an error function like shape? In order to tackle this question, a "real" profile needs to be measured experimentally from the deposited samples. An "effective" experimental in-depth Ru concentration profile can be determined from the background at energies lower than the Ru surface peak ("tail" to the peak) in the LEIS spectrum.

The "low-energy tail" from the $\mathrm{Ru}$ peak originates from backscattering of $\mathrm{He}^{0}$ neutrals from $\mathrm{Ru}$ atoms below the surface, since practically all $\mathrm{He}^{+}$ions are neutralized upon penetrating below the outermost atomic layer. The intensity of the "tail" signal depends both on the amount of $\mathrm{Ru}$ atoms below the surface, as well as on the reionization probability for $\mathrm{He}^{0}$ neutrals scattered below the $\mathrm{Ru}$ surface. This re-ionization probability depends on the velocity of the scattered particles and the presence of 
electronegative species on the sample surface. ${ }^{270}$ The re-ionized $\mathrm{He}^{0}$ neutrals scattered from $\mathrm{Ru}$ atoms below the surface carry less energy than projectiles scattered at the surface due to (inelastic) collision processes. ${ }^{276}$

The energy loss $<\Delta E>$ for the backscattering of He atoms in a certain Ru depth $d$ can be calculated as the difference between the surface binary collision peak at $\sim 2500$ $\mathrm{eV}$, and the energy of the backscattered projectile from this depth $d$. The energy of the backscattered $\mathrm{He}$ atoms from the deepest $\mathrm{Ru}$ containing layer can be experimentally determined as the intersection between the background intensity at low energy and the low energy start of the $\mathrm{Ru}$ "tail". As an example, the backscattered He energies from the deepest $\mathrm{Ru}$ containing layer for the 0.7 and $4.7 \mathrm{~nm} \mathrm{Ru}$ samples are $2110 \pm 50$ and $1240 \pm 150 \mathrm{eV}$, respectively (as pointed out by dashed arrows in Fig. 5.2). Comparing these values to the Ru peak position, an energy loss $<\Delta E>$ of $395 \pm 50$ and $1265 \pm 150 \mathrm{eV}$ is calculated for the respective 0.7 and $4.7 \mathrm{~nm}$ Ru samples. One can then extract the Ru depth $d$ at which the projectile is backscattered, assuming an energy independent stopping power $S$ for Ru and taking into account the instrument geometry by ${ }^{344}$

$$
d=\frac{<\Delta E>}{2.2 \times S} .
$$

The stopping power for $3 \mathrm{keV}$ He on Ru can be determined using SRIM software. ${ }^{166}$ The obtained value for this parameter is $68 \pm 2 \mathrm{eV} / \mathrm{nm}$. This value has two main contributions: the electronic energy loss (to the target electrons) and the nuclear energy loss (to the target nuclei). Another electronic energy loss associated with the recoiling of the target atoms is not considered in the simulation.

The depth $d$ where the deepest Ru atoms are located (from now on named lowest $\mathrm{Ru}$ depth) is then calculated by Eq. (5.6) for the 0.7 and $4.7 \mathrm{~nm} \mathrm{Ru}$ example samples, resulting in $2.6 \pm 0.3$ and $8.5 \pm 1.0 \mathrm{~nm}$, respectively. These large values for the lowest $\mathrm{Ru}$ depth compared to the Ru deposited amount denote an important intermixing. Note that this method for obtaining the lowest $\mathrm{Ru}$ depth $d$ is only valid if the energy of the backscattered He atoms from this depth $d$ is higher than the reionization threshold. The reionization threshold for $\mathrm{Ru}$ is $600 \mathrm{eV},{ }^{345}$ which corresponds to the low-energy onset of the $\mathrm{Ru}$ "tail" in case of a bulk Ru sample. ${ }^{270}$ For the thickest $4.7 \mathrm{~nm} \mathrm{Ru}$ sample, the energy of the lowest $\mathrm{Ru}$ depth $d$ is $1265 \pm 150 \mathrm{eV}$ (Fig. 5.2), much higher than the reionization value. In addition, this method can only be applied if the projected range of the $\mathrm{He}^{+}$ions is long enough that they can scatter from the lowest $\mathrm{Ru}$ depth $d$, travel back towards the $\mathrm{Ru}$ surface, and finally reach the detector. The calculated projected ranges for $3 \mathrm{keV} \mathrm{He}{ }^{+}$ions in $\mathrm{Ru}$ and $\mathrm{Si}$ are $9.5 \pm 0.5 \mathrm{~nm}$ and $32 \mathrm{~nm}$, respectively, using SRIM software. ${ }^{166}$ This means that our approach for determining the lowest Ru depth $d$ may not be reliable for deposited amounts larger than $\sim 4$ to $5 \mathrm{~nm}$ of $\mathrm{Ru}$. But in practice the measured energy for the lowest Ru depth $d$ still shifts towards lower energies, even for 
the thickest $4.7 \mathrm{~nm} \mathrm{Ru}$ sample (see Fig. 5.3(a)). Apparently, the projected range of ions in $\mathrm{Ru}$ seems underestimated by SRIM software. Thus, $d$ values can still be experimentally determined for the thickest $4.7 \mathrm{~nm} \mathrm{Ru}$ sample. Apart from these two considerations, the influence of straggling could be a last concern for the implementation of our method. For thicker layers, it could be expected that the lower part of the "tail" widens due to straggling of ions in matter. However, in practice we do not observe significant widening of the Ru "tail" with increasing Ru thickness (see Fig. 5.3(a)), such that it can be assumed that the width of the "tails" is dominated by the intermixing of both materials, and not by ion straggling.

From the lowest Ru depth $d$ described by Eq. (5.6), we can determine an "effective" experimental concentration profile of the $\mathrm{Ru}$ atoms below the surface. This "effective" concentration profile can be directly obtained by plotting the experimental $\mathrm{Ru}$ surface coverage $C^{\text {surf }}$ against the lowest $\mathrm{Ru}$ depth $d$ for each deposited amount of $\mathrm{Ru}$. The experimental surface concentration $C$ (or surface coverage $C^{\text {surf }}$ ) obtained before in Sec. 5.3.1 is depicted in Fig. 5.11 (squares) against the lowest Ru depth $d$ (top axis), and the previously simulated in-depth Ru concentration $C$ in Fig. 5.10(a) is also plotted in Fig. 5.11 (line) as a function of $\mathrm{Ru}$ depth $z$ (bottom axis) for $\mathrm{Ru}$ grown on (a) $\mathrm{SiO}_{2}$, (b) $\mathrm{SiN}$ and (c) Si. Note that the top axis corresponding to the lowest $\mathrm{Ru}$ depth $d$ displays the values in reverse order since $d$ represents the distance of the surface from the start of the interface (deepest $\mathrm{Ru}$ containing layer). Also the top axis is shifted in the horizontal direction in order to compare experimental to simulated data.

Fig. 5.11 shows a good overlap between the "effective" experimental concentration profile (squares) and the simulated concentration profile (line) for $\mathrm{Ru}$ grown on $\mathrm{SiO}_{2}$ (a), $\mathrm{SiN}$ (b), and $\mathrm{Si}$ (c). Although it is difficult to determine the initial position of the "effective" experimental profile (at $C=0$ ) on the simulated profile, the shape of both profiles is matching within the error-bars to an error function like shape. This good agreement shows that the profile obtained from the surface LEIS signal indeed matches the "real" interface profile, confirming that no significant interdiffusion takes place after deposition for low-temperature magnetron sputtered $\mathrm{Ru}$ on a-Si, $\mathrm{SiN}$ and $\mathrm{SiO}_{2}$ layers. 

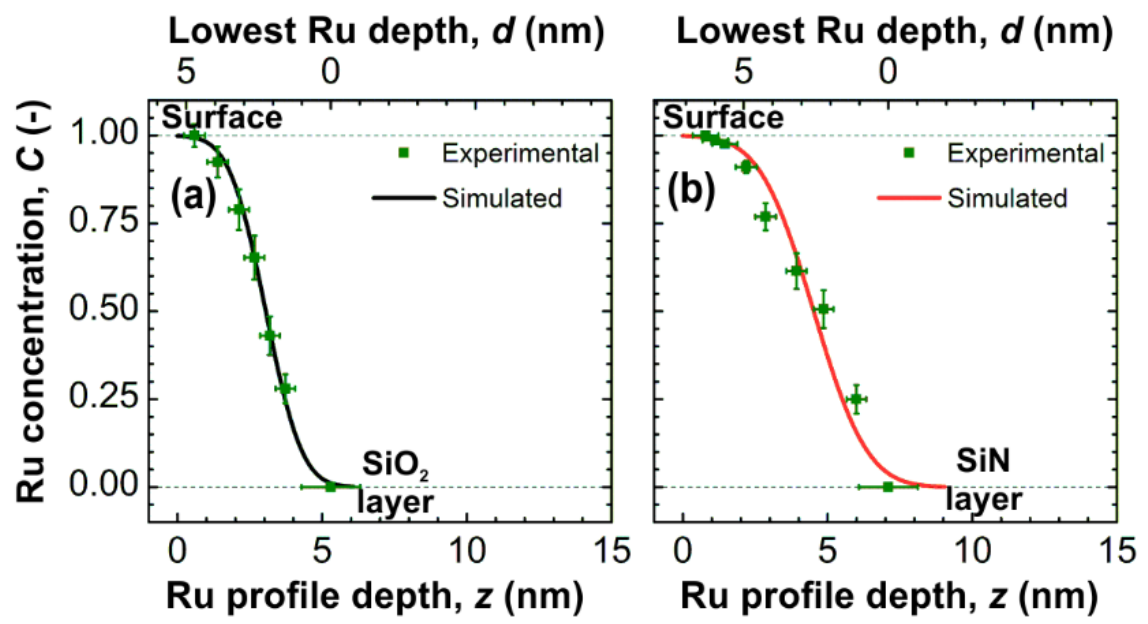

Lowest Ru depth, $d(\mathrm{~nm})$

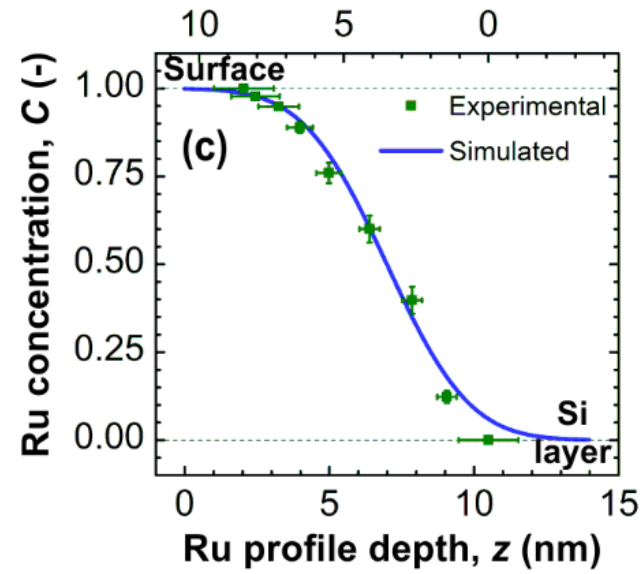

Fig. 5.11. Experimental surface concentration $C$ (or surface coverage $C^{\text {suf }}$ ) (solid squares) as a function of lowest Ru depth $d$ (top axis), and simulated in-depth Ru concentration $C$ (solid lines) as a function of $\mathrm{Ru}$ depth $z$ (bottom axis) for Ru grown on (a) $\mathrm{SiO}_{2}$, (b) $\mathrm{SiN}$ and (c) $\mathrm{Si}$. 


\subsubsection{Surface coverages, closed layer determination and in-depth $\mathrm{Si}$ profile for Si on Ru}

The large amount of $\mathrm{Ru}$ (about $4.7 \mathrm{~nm}$ ) needed to close the $\mathrm{Ru}$ layer for our $\mathrm{Ru}$-on-

Si system compared to other metal-silicon systems, ${ }^{346,347}$ has raised some doubts about the causes of this huge intermixing. A number of factors may contribute to the total intermixing process, such as sputtering induced intermixing during $\mathrm{Ar}^{+}$bombardment, diffusion based intermixing between layers, availability of both ad-atoms and substrate atoms during deposition, and Si surface segregation. Some of these factors should depend on the deposition sequence of layers. To investigate this dependence, we compare the $\mathrm{Ru}$-on-Si system to the reverse system where $\mathrm{Si}$ is grown onto $\mathrm{Ru}$.

Si layers with various thicknesses are grown on $5.0 \mathrm{~nm}$ Ru layers onto $\mathrm{Si}(100)$ substrates, and analyzed by LEIS. Following the previous data analysis described in Sec. 5.3.1 for $\mathrm{Ru}$ on $\mathrm{Si}$, the surface coverages for $\mathrm{Si}$ on $\mathrm{Ru}$ are determined. In addition, the simulated and the "effective" experimental in-depth Si concentration profiles are also obtained from the determined Si surface coverage as described in Sec. 5.3.2. Note that for this last "effective" experimental Si profile, the lowest $\mathrm{Si}$ depth $d$ needs to be calculated.

To calculate this depth $d$ where the deepest $\mathrm{Si}$ atoms are located, the procedure described in Sec. 5.3.2 for the lowest Ru depth must be redefined for Si. This lowest Si depth $d$ is also equivalent to the Ru depth at which the $\mathrm{Si}-\mathrm{Ru}$ interface starts. The energy of the backscattered $\mathrm{He}$ atoms from $\mathrm{Si}$ atoms at this $\mathrm{Ru}$ depth can be experimentally determined as the point where the Ru "tail" reaches a constant level when going to lower energies from the high energy start of the $\mathrm{Ru}$ "tail". As an example, for the $0.9 \mathrm{~nm} \mathrm{Si}$ sample a value of $2184 \pm 50 \mathrm{eV}$ is obtained for this energy (as pointed out in Fig. 5.12(a)). Then, the energy loss $\langle\Delta E>$ for He atoms at this depth $d$ can be similarly calculated as the difference between the surface $\mathrm{Ru}$ peak at $\sim 2500 \mathrm{eV}$, and the energy of the backscattered projectile at this depth $d$. Finally, the lowest Si depth $d$ can be calculated for each deposited Si thickness by means of Eq. (5.6) from Sec. 5.3.2, when considering a stopping power $S$ of $45 \pm 2 \mathrm{eV} / \mathrm{nm}$ for $3 \mathrm{keV} \mathrm{He}$ on $\mathrm{Si}$ (according to SRIM calculations ${ }^{166}$ ). Note that both considerations for the applicability of our method (as discussed in Sec. 5.3.2) are also fulfilled for this $\mathrm{Si}-\mathrm{Ru}$ system. The reionization threshold for $\mathrm{Si}$ is $400 \mathrm{eV},{ }^{345}$ much lower than the $2184 \pm 50 \mathrm{eV}$ energy value which corresponds to the lowest Si depth $d$ for the thickest 0.9 nm Si sample (Fig. 5.12(a)). Also, this thickest Si layer is small compared to the previously mentioned projected range of the ions.

Fig. 5.12(a) shows all LEIS spectra for Si on Ru, and Fig. 5.12(b) presents their respective surface coverages for $\mathrm{Ru}$ and $\mathrm{Si}$. Fig. 5.13(a) displays the simulated in-depth 
concentration profiles $C(z)$ for $\mathrm{Si}$ on $\mathrm{Ru}$ and for $\mathrm{Ru}$ on $\mathrm{Si}$, Fig. 5.13(b) represents the fits of their respective simulated surface concentrations $C(t)$ to their experimental surface coverage $C^{\text {surf }}$, and Fig. 5.13(c) presents the previous simulated Si concentration profile $C(z)$ shown in Fig. 5.13(a) plotted together with the "effective" experimental Si concentration profile.

(a)

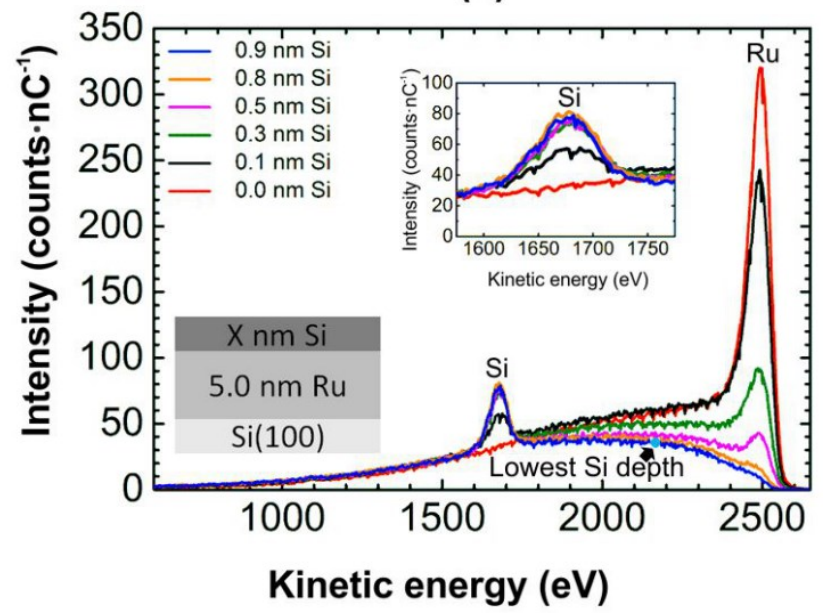

(b)

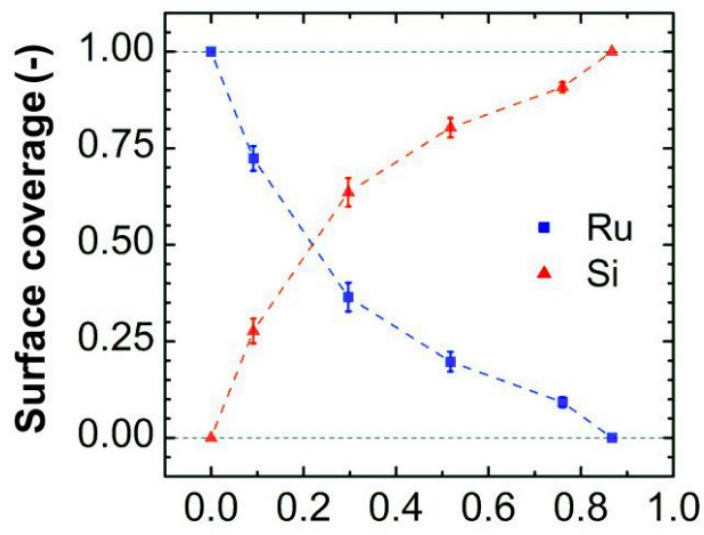

Deposited amount of Si (nm)

Fig. 5.12. (a) LEIS spectra of Si on Ru. Right-top inset displays the zoom of the Si peak. Leftbottom inset shows the layered model of the deposited structure. The lowest $\mathrm{Si}$ depth for $0.9 \mathrm{~nm} \mathrm{Si}$ is also pointed out. (b) Surface coverages for $\mathrm{Ru}$ (squares) and $\mathrm{Si}$ (triangles) for $\mathrm{Si}$ on $\mathrm{Ru}$. The dashed lines through the data points are guides for the eye. 
(a)

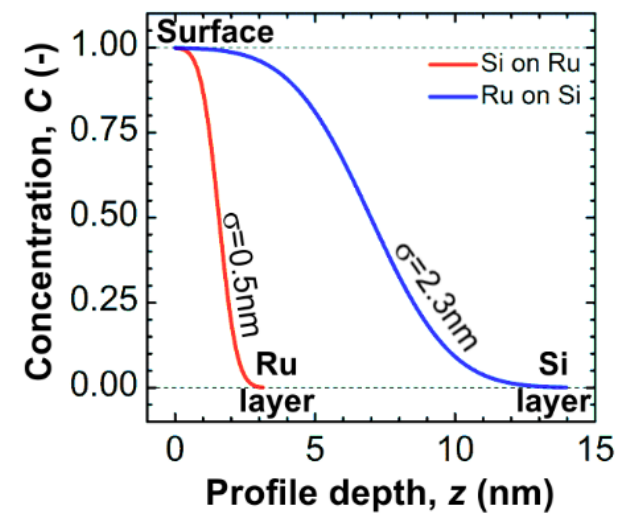

(b)

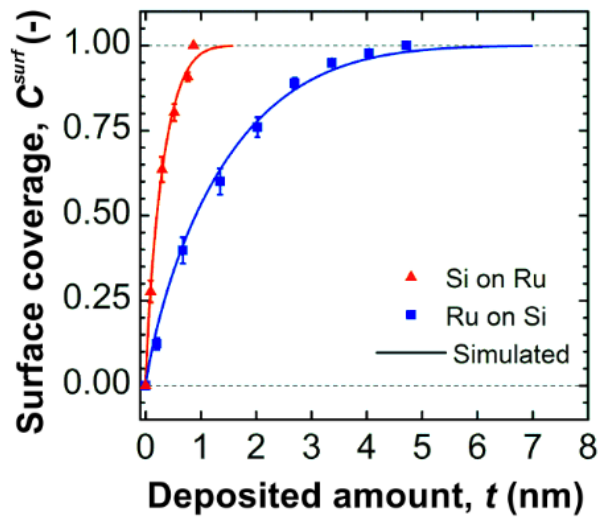

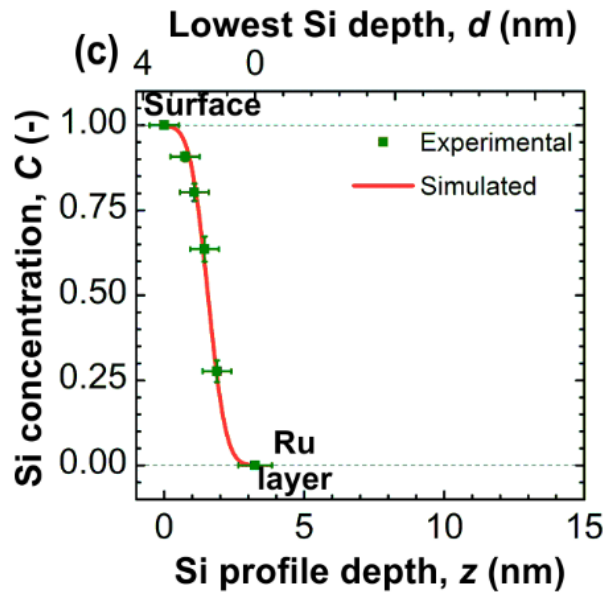

Fig. 5.13. (a) Simulated in-depth concentration profiles $C(z)$ for $\mathrm{Si}$ on $\mathrm{Ru}$ and for $\mathrm{Ru}$ on $\mathrm{Si}$. The characteristic profile widths $\sigma$ are also shown in (a). (b) Surface concentration $C^{\text {surf }}$ plotted against experimental (symbols) and simulated (lines) deposited thickness $t$ for Si on Ru (triangles) and Ru on Si (squares). (c) Simulated in-depth Si concentration profile depicted in (a, red line) and, "effective" experimental Si concentration profile (squares) for $\mathrm{Si}$ on $\mathrm{Ru}$. Note that the top in (c) corresponding to the lowest Si depth $d$ displays the values in reverse order and is shifted in the horizontal direction in order to compare experimental to simulated data. 
The $\mathrm{Ru}$ surface peak vanishes totally after about $0.9 \mathrm{~nm}$ deposited Si (see blue spectrum in Fig. 5.12(a)) when Si coverage is 100\% (Fig. 5.12(b)), which means that about $0.9 \mathrm{~nm} \mathrm{Si}$ are needed to close the layer for $\mathrm{Si}$ on $\mathrm{Ru}$ compared to the $4.7 \mathrm{~nm} \mathrm{Ru}$ needed to close the layer for $\mathrm{Ru}$ on $\mathrm{Si}$. The in-depth concentration profile for $\mathrm{Si}$ on $\mathrm{Ru}$ is about 5 times sharper than the Ru on Si one (see $\sigma$ values in Fig. 5.13(a)). Similarly as for $\mathrm{Ru}$ on $\mathrm{Si}$, for $\mathrm{Si}$ on $\mathrm{Ru}$ there is a good agreement between simulated surface concentrations and experimental surface coverages (Fig. 5.13(b), red line and triangles, respectively), and a good match between simulated $\mathrm{Si}$ concentration profile and "effective" experimental Si concentration profile (Fig. 5.13(c), red line and squares, respectively). This means that for this reverse system an error function model is also valid to describe the $\mathrm{Si}-\mathrm{Ru}$ interface, and confirms that our method based on the reconstruction of the in-depth profiles from the surface coverages can also be applied in the Si-on-Ru system.

The huge difference between Ru-on-Si and Si-on-Ru systems confirms that there are several factors contributing to the total intermixing process which are different for a reversed layer deposition sequence. We propose Si surface segregation, observed for Ru deposited on $\mathrm{Si}$, as one of these factors that should be fundamentally different for the two systems, and we investigate it in more detail.

Surface segregation of Si during growth by room-temperature magnetron sputtering has been observed for several systems. ${ }^{348,349}$ For instance, as deposited magnetron sputtered amorphous $\mathrm{NbSi}$ thin films exhibited about $11.5 \%$ of Si surface segregation when comparing surface (or near-surface) Si concentration to bulk Si concentration. ${ }^{348}$ The surface segregation effect for the Ru-on-Si system could be explained by simple thermodynamics when considering the difference in standard surface free energy $\gamma^{o}$ between $\mathrm{Si}$ and $\mathrm{Ru}$ surfaces $\left(\gamma_{\mathrm{Si}}^{o}=1.1 \text { and } \gamma^{o}{ }_{\mathrm{Ru}}=3.4 \mathrm{~J} \cdot \mathrm{m}^{-2} \text { at } 25^{\circ} \mathrm{C}\right)^{350}$. For the Si-on-Ru system, this segregation effect should not be present since the surface free energy is already minimized by having Si on the top.

If there is $\mathrm{Si}$ surface segregation on the Ru surface the concentration of the surface is no longer equal to the concentration of the layer just below the surface. For this purpose, we qualitatively investigate the in-depth Ru signal ("tail" to the Ru peak) in the LEIS spectra while comparing to the Si surface signal. If Si surface segregation is present, the in-depth Ru signal would already appear saturated for a Ru thickness below the closing of the layer, while the Si surface peak would still be visible.

Fig. 5.14 displays the LEIS spectra of $\mathrm{Ru}$ grown on $\mathrm{Si}$ (a), $\mathrm{SiN}$ (b) and $\mathrm{SiO}_{2}$ for different Ru thicknesses near to the closing of the layer, in order to compare in-depth $\mathrm{Ru}$ and surface Si signals. For the Ru on Si system, the in-depth Ru signal just below the surface seems to saturate at about $2.7 \mathrm{~nm}$ Ru thickness by the appearance of a plateau close to the Ru surface peak in the LEIS spectrum while there is still a prominent $\mathrm{Si}$ 
surface peak (Fig. 5.14(a), brown line). This confirms Si surface segregation and suggests that just above $2.7 \mathrm{~nm}$ the grown layer is almost bulk Ru and it is considered to be practically closed. The Si surface coverage at $2.7 \mathrm{~nm} \mathrm{Ru}$ is $11.1 \pm 1.6 \%$ (from Fig. 5.6(d) in Sec. 5.3.1, triangles), which mostly corresponds to the amount of Si segregated towards the $\mathrm{Ru}$ surface. The plateau in the $\mathrm{Ru}$ "tail" is maintained for $\mathrm{Ru}$ thicknesses larger than $2.7 \mathrm{~nm}$ but the surface segregation effect slightly reduces with the increase of the $\mathrm{Ru}$ thickness and vanishes totally when the layer fully closes at about $4.7 \mathrm{~nm} \mathrm{Ru}$ as observed by the decrease and disappearance of the Si surface peak in Fig. 5.14(a). A study by Yan, et al. ${ }^{335}$ showed that the direct deposition of Ru on amorphous Si leads to the formation of about $2 \mathrm{~nm}$ thick ruthenium silicide. This value was determined by an ex situ TEM analysis of a $\mathrm{Ru} / \mathrm{Si}$ multilayer structure. It should be noted that TEM analysis may not detect such a small amount of segregated $\mathrm{Si}$ which we could determine with our high-sensitivity LEIS setup. Assuming that about $2.7 \mathrm{~nm}$ Ru required to reach "bulk Ru" conditions just below the surface corresponds mostly to the silicide formation process, the $\sim 2 \mathrm{~nm}$ difference with respect to the $4.7 \mathrm{~nm} \mathrm{Ru}$ needed to fully close the layer, is attributed to Si surface segregation.

For the $\mathrm{Ru}$ on $\mathrm{SiN}$ system there is also $\mathrm{Si}$ surface segregation since the in-depth $\mathrm{Ru}$ signal seems to saturate at about $2.0 \mathrm{~nm} \mathrm{Ru}$ thickness (see plateau appearing in Fig. 5.14(b), green spectrum) while the Ru surface signal increases until $3.2 \mathrm{~nm}$ (as observed in Fig. 5.3(b) from Sec. 5.3.1). Since the deposited $\mathrm{SiN}_{\mathrm{x}}$ layer is sub-stoichiometric and silicon-rich, it is possible that some free $\mathrm{Si}$ from this $\mathrm{SiN}_{\mathrm{x}}$ layer segregates up towards the $\mathrm{Ru}$ surface. Compared to $\mathrm{Ru}-\mathrm{on}-\mathrm{Si}$, for this system there is a difference of only $\sim 1.2$ $\mathrm{nm}$ between the point when the Ru layer closes and the saturation of the in-depth $\mathrm{Ru}$ signal. This means that the segregation effect of $\mathrm{Si}$ is reduced almost by half when $\mathrm{Si}$ is passivated by $\mathrm{N}_{2}$.

For the $\mathrm{Ru}$ on $\mathrm{SiO}_{2}$ system, the $\mathrm{Si}$ surface segregation effect seems not to be present since the in-depth $\mathrm{Ru}$ signal saturates just at the moment when the Si surface peak completely vanishes at about $2.0 \mathrm{~nm}$ and the Ru layer closes (Fig. 5.14(c), blue spectrum). The absence of $\mathrm{Si}$ surface segregation in the $\mathrm{SiO}_{2}$ system might be due to the fact that the $\mathrm{Si}$ is strongly bound to oxygen. ${ }^{38}$ Even though some of the oxygen from the $\mathrm{SiO}_{2}$ reacts with $\mathrm{Ru}$ and segregates to the surface, surface segregation of $\mathrm{Si}$ is not observed, probably because $\mathrm{RuO}_{\mathrm{x}}$ formation at the surface is thermodynamically more favorable than $\mathrm{RuSi}_{\mathrm{x}}$ formation. ${ }^{38}$ 
(a)

(b)
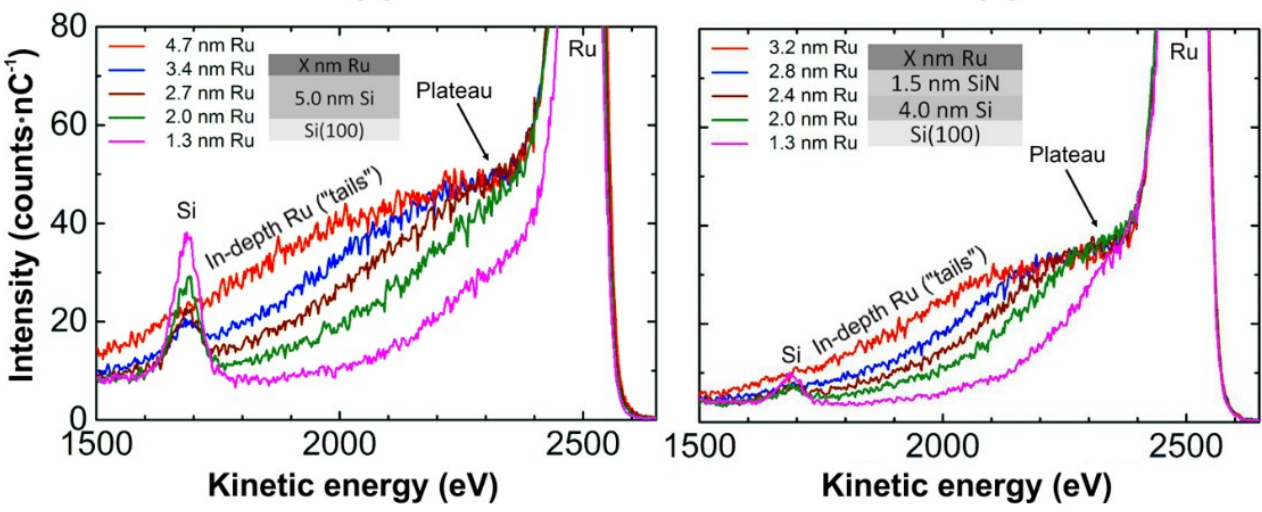

(c)

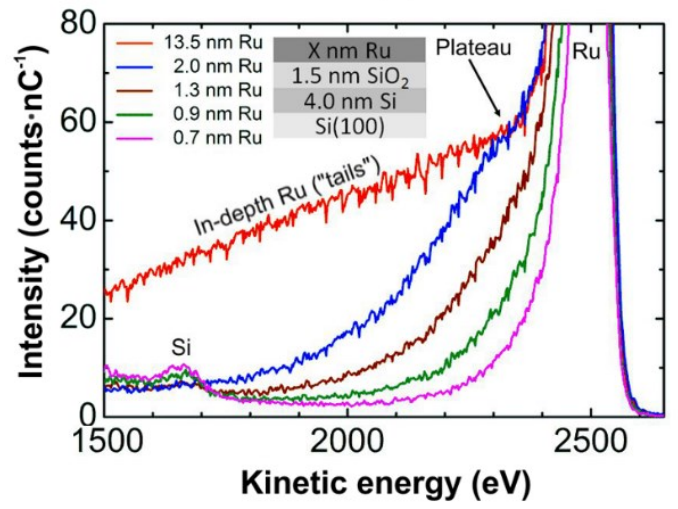

Fig. 5.14. LEIS spectra of (a) $\mathrm{Ru}$ on $\mathrm{Si}$, (b) $\mathrm{Ru}$ on $\mathrm{SiN}$, and (c) $\mathrm{Ru}$ on $\mathrm{SiO}_{2}$ for different thicknesses near to the closing of the layer. Top-middle insets in (a), (b) and (c) show the layered models of the deposited structures. Note that the saturation level of the in-depth Ru ("tails") signal is achieved by the formation of a plateau close to the Ru surface peak. 
Under surface segregation conditions, the surface concentration differs from the concentration just below the surface. We must take into account the surface segregation effect when reconstructing the in-depth profiles from the surface peaks information. This is easily performed for the oxygen segregation process present in $\mathrm{Ru}$-on- $\mathrm{SiO}_{2}$, where a small fraction of surface segregated oxygen stays on the surface, even for large $\mathrm{Ru}$ thicknesses. This oxygen surface segregation effect goes independently from the silicide formation process and it only depends on the Ru surface coverage. In contrast for the $\mathrm{Ru}$ on $\mathrm{Si}$ or SiN systems, the Si surface segregation depends on the Ru surface coverage and it also depends on the silicide formation since both processes are connected and occurring simultaneously. Therefore, it is not straightforward to fully separate both contributions.

Looking in more detail to the overlap between the experimental and the simulated in-depth Ru concentration profiles for Ru on SiN and Ru on Si (Figs. 5.11(b) and 5.11(c), respectively), we can see that there is an appreciable mismatch between profiles. Distinctly for $\mathrm{Ru}$ on $\mathrm{SiO}_{2}$ and $\mathrm{Si}$ on $\mathrm{Ru}$, both profiles match very well within the error bars as depicted in Figs. 5.11(a) and 5.13(c), respectively. This is due to the fact that for $\mathrm{Ru}$ on $\mathrm{Si}$ and $\mathrm{Ru}$ on $\mathrm{SiN}$ there is $\mathrm{Si}$ surface segregation taking place and for $\mathrm{Ru}$ on $\mathrm{SiO}_{2}$ and $\mathrm{Si}$ on $\mathrm{Ru}$ there is no apparent $\mathrm{Si}$ surface segregation (when the $\mathrm{O}$ surface segregation effect is extracted from the $\mathrm{Ru}$ on $\mathrm{SiO}_{2}$ growth). A deeper investigation would be needed to fully separate the surface segregation effect from the silicide formation process for Ru-on-Si and Ru-on-SiN systems, but goes beyond the scope of this chapter.

Although there is a strong Si surface segregation effect for $\mathrm{Ru}$ on $\mathrm{Si}$, which seems not to be present for the reverse system, still there is a difference in intermixing when comparing the $2.7 \mathrm{~nm} \mathrm{Ru}$ (on $\mathrm{Si}$ ) with the $0.9 \mathrm{~nm} \mathrm{Si}$ (on $\mathrm{Ru}$ ) needed to form the silicide. For the $\mathrm{Ru}$ on $\mathrm{Si}$, part of this $2.7 \mathrm{~nm} \mathrm{Ru}$ may be related to Si surface segregation that occurs simultaneously with the silicide formation process. In addition, there are other processes that could affect the overall intermixing process, which can differ from one system to the other, such as sputtering induced intermixing due to energetic particles from the magnetron plasma, inter-diffusion (possibly associated to the crystallinity of the substrate layers), or availability of both $\mathrm{Si}$ and $\mathrm{Ru}$ atoms which leads to a different silicide being formed.

Sputtering induced intermixing might occur due to the impact of $\mathrm{Ar}^{+}$ions or neutrals reflected on the target. To evaluate this option, SRIM simulations are performed, bombarding $\mathrm{Ar}^{+}$ions at normal incidence on $\mathrm{Ru}$ (300 eV initial energy) and Si targets (400 eV energy) (as described in ${ }^{166}$ ). The results show about $15.7 \%$ backscattered $\mathrm{Ar}^{+}$ ions from $\mathrm{Ru}$ target and only $0.1 \%$ from Si target. In the ion energy distribution of the backscattered $\mathrm{Ar}^{+}$ions from $\mathrm{Ru} 70 \%$ of the ions is in the range between 0 to $40 \mathrm{eV}$ while $30 \%$ of the ions are between 40 to $100 \mathrm{eV}$. In contrast for $\mathrm{Si}$, the backscattered $\mathrm{Ar}^{+}$ energies are lower and with values between 0 to $5 \mathrm{eV}$ for all backscattered ions. There 
are some differences between $\mathrm{Ru}$ and $\mathrm{Si}$ but, since both backscattered $\mathrm{Ar}^{+}$energies are low, the sputtering processes due to ballistic effects can be considered negligible for explaining the difference in intermixing between $\mathrm{Ru}$-on-Si and $\mathrm{Si}$-on-Ru systems.

When considering the crystal structure of the initial deposited $\sim 5 \mathrm{~nm}$ substrate layers, the Si presents an amorphous structure while $\mathrm{Ru}$ is already polycrystalline. ${ }^{325,351}$ An amorphous Si layer might intermix easier with $\mathrm{Ru}$ ad-atoms, than for $\mathrm{Si}$ ad-atoms to interact with a crystalline Ru layer since a disordered matrix has more mobility of its atoms than an ordered structure where the atoms are strongly bounded within the "rigid" lattice. As a result, Ru could intermix more on top of $\mathrm{Si}$ than $\mathrm{Si}$ on top of $\mathrm{Ru}$. This phenomenon has already been observed by Yulin et al. ${ }^{59}$ in the growth of $\mathrm{Mo} / \mathrm{Si}$ multilayers where there was a larger intermixing in the Mo-Si interface compared to the Si-Mo interface, associated to the crystallinity of the Mo layers. This interfacial asymmetry was no longer present when the Mo layers were amorphous. For the asymmetric case which is similar to our study system, the Mo-Si interface was about 1.2 $\mathrm{nm}$ and the Si-Mo interface was about $0.6 \mathrm{~nm}$. This factor of half is also observed in our systems, when compared Ru-on-Si to Si-on-Ru, and judging completion of the silicide layer from the "tail" signal. For Mo-Si system the silicide formed for both interfaces was $\mathrm{MoSi}_{2}$. In our case, the stoichiometry of the formed silicide was not studied. According to Zhang et al., ${ }^{168}$ the more thermodynamically stable silicides are (in order of increasing stability) $\mathrm{RuSi}_{2}$, followed by $\mathrm{Ru}_{2} \mathrm{Si}_{3}$, and $\mathrm{RuSi}$. The difference in formation enthalpy between those silicides is in the order of $0.1 \mathrm{eV} /$ atom. Therefore, it is also possible that initially different silicides are formed for each interface according to the availability of $\mathrm{Ru}$ and $\mathrm{Si}$ atoms. The silicide formation process as any chemical reaction is controlled by the limiting reagent, which is the reactant that limits the amount of products formed. ${ }^{212}$ For Ru-on-Si, the limiting reagent is $\mathrm{Ru}$ and for the reverse system is $\mathrm{Si}$, when considering the $\sim 5 \mathrm{~nm} \mathrm{Si}$ and $\mathrm{Ru}$ substrate layers to be in excess. If we deposit $\mathrm{Ru}$ on $\mathrm{Si}$, it may be that initially $\mathrm{RuSi}_{2}$ is formed. When more $\mathrm{Ru}$ is deposited, there may be a driving force for the $\mathrm{Si}$ atoms in the $\mathrm{RuSi}_{2}$ to diffuse towards the surface, such that a more stable RuSi is formed. This can be another origin of a wider Ru-on-Si interface compared to the reverse system. Further research is needed to establish the stoichiometry of the silicides formed, which can help to fully understand the difference between Ruon-Si and Si-on-Ru systems. 


\subsection{Discussion}

Based on the previous data analysis presented in Secs. 5.3.1 and 5.3.3, we propose the following mechanisms involved in the growth of $\mathrm{Ru}$ onto amorphous $\mathrm{Si}, \mathrm{SiN}$ and $\mathrm{SiO}_{2}$ layers.

For the Ru-on-Si system, there are two competing diffusion mechanisms simultaneously occurring in the first stages of growth until the $\mathrm{Ru}$ thickness reaches about $2.7 \mathrm{~nm}$, coinciding with the moment when the layer is almost closed. During this initial period, $\mathrm{Ru}$ atoms arriving on the $\mathrm{Si}$ surface, interdiffuse with $\mathrm{Si}$ and react, forming $\mathrm{RuSi}_{\mathrm{x}}$. At the same time, a small amount of $\mathrm{Si}$ atoms segregates towards the surface and reacts with the $\mathrm{Ru}$, forming surface silicide. Although there is surface segregation of $\mathrm{Si}$, the main process at this stage is the interfacial silicide formation. Approaching $2.7 \mathrm{~nm}$ $\mathrm{Ru}$, the intermixing slows down, likely due to slow diffusion of $\mathrm{Si}$ atoms from the $\mathrm{Si}$ underlayer through the formed interlayer. The only remaining mechanism above $2.7 \mathrm{~nm}$ is the diffusion (segregation) of $\mathrm{Si}$ from the intermixed layer upwards towards the surface. Upon further deposition, the rate of $\mathrm{Ru}$ ad-atom arrival overcomes the rate of $\mathrm{Si}$ diffusion to the surface until no more $\mathrm{Si}$ is observed at $4.7 \mathrm{~nm}$, when the layer fully closes. At this point, Ru is covering homogeneously all the surface and new incorporated $\mathrm{Ru}$ will grow continuously on top of this layer.

For the $\mathrm{Ru}$ on $\mathrm{SiN}$ system, the two co-existing mechanisms observed for $\mathrm{Ru}$ on $\mathrm{Si}$ are also present in the initial phases of growth until $\mathrm{Ru}$ approaches a thickness of about $2.0 \mathrm{~nm}$ when the Ru concentration is almost bulk. Coinciding with the previous system, the main occurring process during this period is the silicide formation at the interface. The interfacial silicide formation seems to ends after $2.0 \mathrm{~nm} \mathrm{Ru}$ and the only mechanism that prevails is the surface segregation of Si. This process decreases when the Ru layer increases its thickness and finally disappears at about $3.2 \mathrm{~nm}$ when $\mathrm{Ru}$ reaches the $100 \%$ concentration. Later on, more added material will continue growing as bulk Ru.

For the $\mathrm{Ru}$ on $\mathrm{SiO}_{2}$ system, the silicate formation is the only mechanism present in the initial stages of growth until a thickness of about $2 \mathrm{~nm} \mathrm{Ru}$ is reached. At this moment the layer is fully closed, and the next deposited Ru will grow homogeneously on the top of this bulk layer. It should be noted that apart from these mechanisms there is a small amount of oxygen segregating on the $\mathrm{Ru}$ surface from the $\mathrm{Ru}-\mathrm{SiO}_{2}$ interface. But it is a constant and independent mechanism that does not interfere in the overall growth process.

It should be noted that the growth mechanisms derived from this LEIS study require the knowledge that our Ru layers grow smoothly on top of all substrates layers, as studied by AFM (see Sec. 5.3.1). This excludes that 3D island growth is the (main) cause for the large amount of Ru needed for forming a closed layer. 


\subsection{Conclusions}

The initial growth of $\mathrm{Ru}$ on amorphous $\mathrm{Si}, \mathrm{SiN}$ and $\mathrm{SiO}_{2}$ has been studied by in vacuo HS-LEIS. This technique allowed an accurate determination of surface coverages and thicknesses required for closing the layer on all substrate materials and also the detection of surface oxygen for $\mathrm{Ru}$ grown on $\mathrm{SiO}_{2}$. In vacuo XPS measurements confirmed the presence of surface oxygen and revealed a constant oxygen surface segregation effect during the growth of this system. Ru forms a closed layer at about 2.0, 3.2 and $4.7 \mathrm{~nm}$ on top of $\mathrm{SiO}_{2}, \mathrm{SiN}$ and $\mathrm{Si}$ layers, respectively. The decrease of this intermixing with increasing passivation was mainly attributed to the thermodynamics involved in the compound formation for all three systems.

In-depth $\mathrm{Ru}$ concentration profiles were reconstructed from the determined $\mathrm{Ru}$ surface coverages for $\mathrm{Ru}$ grown on $\mathrm{Si}, \mathrm{SiN}$ and $\mathrm{SiO}_{2}$. The good overlap between experimental and simulated $\mathrm{Ru}$ concentration profiles confirmed the use of an error function profile to describe the $\mathrm{Ru}$ interface and showed that no significant interdiffusion takes place after deposition.

The large intermixing $(4.7 \mathrm{~nm})$ for the $\mathrm{Ru}$ on $\mathrm{Si}$ system when compared to the 0.9 $\mathrm{nm}$ value for the reverse system ( $\mathrm{Si}-\mathrm{on}-\mathrm{Ru}$ ), could be predominantly explained by the strong Si surface segregation detected for Ru-on-Si, which was absent for Si-on-Ru. This effect should be related to the difference in surface free energy between $\mathrm{Si}$ and $\mathrm{Ru}$ surfaces. Si surface segregation was also observed for Ru-on-SiN. Contrarily, for Ru-on$\mathrm{SiO}_{2}$, $\mathrm{Si}$ surface segregation was not observed, most probably due to the fact that $\mathrm{Si}$ is strongly bound to oxygen and because $\mathrm{RuO}_{\mathrm{x}}$ formation at the surface is thermodynamically more favorable than $\mathrm{RuSi}_{\mathrm{x}}$ formation.

\section{Acknowledgments}

The authors are grateful to Professor Hidde Brongersma (Eindhoven University of Technology) and Andrey Zameshin (University of Twente) for their suggestions and interesting discussions on the LEIS data analysis. This work is part of the research programme 'Controlling photon and plasma induced processes at EUV optical surfaces (CP3E)' of the 'Stichting voor Fundamenteel Onderzoek der Materie (FOM)', which is financially supported by the 'Nederlandse Organisatie voor Wetenschappelijk Onderzoek (NWO)'. The CP3E programme is carried out in the Industrial Focus Group XUV Optics, supported by Carl Zeiss SMT, ASML, PANalytical, DEMCON, SolMates, TNO, and the Province of Overijssel. 


\title{
6. Intermixing and thermal oxidation of $\mathrm{ZrO}_{2}$ thin films grown on a-Si, $\mathrm{SiN}$ and $\mathrm{SiO}_{2}$ by metallic and oxidic mode magnetron sputtering
}

\begin{abstract}
The initial growth of DC sputtered $\mathrm{ZrO}_{2}$ on top of a-Si, $\mathrm{SiN}$ and $\mathrm{SiO}_{2}$ layers has been studied by in vacuo high-sensitivity low energy ion scattering (HS-LEIS) for two gas deposition conditions with different oxygen content (high-O and low-O conditions). This unique surface sensitive technique allowed the determination of surface composition and thicknesses required to close the $\mathrm{ZrO}_{2}$ layer on all three substrates for both conditions. The $\mathrm{ZrO}_{2}$ layer closes similarly on all substrates due to more favorable enthalpies of formation for $\mathrm{ZrO}_{2}$ and $\mathrm{ZrSiO}_{4}$, resulting in passivation of the Si from the substrate. However, this layer closes at about half of the thickness $(\sim 1.7 \mathrm{~nm})$ for low-O conditions due to less oxidative conditions and less energetic particles arriving at the sample, which leads to less intermixing via silicate formation. In contrast, for high-O conditions, there is more $\mathrm{ZrSiO}_{4}$ and/or $\mathrm{SiO}_{\mathrm{x}}$ formation, giving more intermixing $(\sim 3.4 \mathrm{~nm})$. In vacuo $\mathrm{X}$ ray photoelectron spectroscopy (XPS) measurements revealed similar stoichiometric $\mathrm{ZrO}_{2}$ layers deposited by both conditions and, a higher interaction of the $\mathrm{ZrO}_{2}$ layer with the underlying a-Si for high-O conditions. In addition, oxygen diffusion through low-O $\mathrm{ZrO}_{2}$ films on a-Si has been investigated by ex situ angular-resolved XPS (AR-XPS) of samples annealed in atmospheric oxygen. For temperatures below $400^{\circ} \mathrm{C}$, no additional oxidation of the underlying a-Si was observed. This, together with the amorphous nature and smoothness of these samples, makes $\mathrm{ZrO}_{2}$ a good candidate as oxidation protective layer on top of a-Si.
\end{abstract}




\subsection{Introduction}

Zirconium oxide thin films $\left(\mathrm{ZrO}_{2}\right)$ present several attractive properties including high thermal and chemical stability, high dielectric constant, large band gap, high hardness, and good optical performance in the near UV-Vis regions (high refractive index and low optical loss). ${ }^{22,24,28}$ These properties make $\mathrm{ZrO}_{2}$ thin films suitable for many applications. For instance, $\mathrm{ZrO}_{2}$ thin films can be used in optical devices such as UV filters and laser mirrors. ${ }^{29,30}$ Apart from other relevant properties, the low reactivity of $\mathrm{ZrO}_{2}$ with $\mathrm{Si}^{32}$ has made $\mathrm{ZrO}_{2}$ an alternative high-K material to $\mathrm{SiO}_{2}$ used as gate dielectric for complementary metal-oxide-semiconductor (CMOS) devices, ${ }^{22,33}$ and as dielectric layer for volatile dynamic random access memories (DRAMs). ${ }^{34}$ This low reactivity of $\mathrm{ZrO}_{2}$ with $\mathrm{Si}$ together with the fact that $\mathrm{Zr}$ forms a very stable and inert oxide to possible external contaminants, ${ }^{37,38}$ have made $\mathrm{ZrO}_{2}$ also a promising candidate as capping layer for optics designed for extreme ultraviolet lithography (EUVL). ${ }^{41,42}$ In the last three applications, oxygen diffusion towards deeper layers is one of the main threats for their performance. Both gate dielectrics and memory devices are usually fabricated at high temperatures under a strong oxidative environment. In such extreme conditions, the poly-Si plug attached to the high-K dielectric layer is oxidized, resulting in a decrease of the effective dielectric constant. ${ }^{22,43}$ Similarly, EUV light induces the cracking of the water vapor present in the lithography vacuum system, generating aggressive oxygen species. ${ }^{352}$ These highly reactive oxygen species lead to oxidation of the Si/Mo multilayer mirror (MLM), causing a reflectance drop. ${ }^{7}$ In these three cases, the $\mathrm{Si}$ layer underneath should not be directly exposed to an oxidative environment. Thus, the $\mathrm{ZrO}_{2}$ protective layer should be a stoichiometric, inert and closed layer that does not allow transport of oxygen through it.

Like other metal oxides, ${ }^{325,334} \mathrm{ZrO}_{2}$ usually crystallizes after a few nanometers of film thickness, allowing $\mathrm{O}$ diffusion through grain boundaries in the film. ${ }^{22,34}$ Besides, grain boundaries can cause undesired current leakage, and the formation of anisotropic crystalline phases can lead to non-uniformities in the dielectric constant, affecting the performance of CMOS and DRAM devices. ${ }^{62,63}$ Thus, the dielectric layer thickness of such devices should be reduced to ensure an amorphous structure. Since oxides highly absorb in the EUV range, ${ }^{7}$ it is also critical to minimize their layer thickness. For instance, a $3 \mathrm{~nm} \mathrm{ZrO}_{2}$ capping layer can cause more than $1 \%$ reflectance loss when applied on a Si-terminated high-reflectance MLM, above the maximum loss permitted for such mirrors. ${ }^{40}$ For all these reasons, an ultrathin amorphous $\mathrm{ZrO}_{2}$ layer is necessary for both electronics and optical applications to act as an effective diffusion barrier with good optical response.

To study the growth of such an ultrathin $\mathrm{ZrO}_{2}$ film, a high surface sensitive technique which allows an accurate investigation of the initial growth stages is needed, 
including the determination of the thickness where the layer closes with Ångström precision. From all surface analysis techniques, low energy ion scattering (LEIS) is considered to be unique for such studies. ${ }^{353}$ This uniqueness relies on the capacity of LEIS to fully separate the information of the outermost surface atomic layer from the layers below the surface, ${ }^{276}$ providing an accurate monitoring of the surface composition during growth of a thin film until it closes. ${ }^{353}$ More details about this technique can be found in ref. ${ }^{270}$.

$\mathrm{ZrO}_{2}$ thin films prepared by chemical vapor deposition, ${ }^{186,187}$ pulsed laser deposition, ${ }^{188,354}$ sol-gel processing, ${ }^{355,356}$ electron beam evaporation, ${ }^{191,357}$ and sputtering, ${ }^{157,195}$ have been reported. It is known that film structure and film properties are influenced by both deposition process and deposition parameters. In the case of reactive sputtering, Venkarataj et al. ${ }^{157}$ revealed a strong influence of the $\mathrm{O}_{2}$ partial pressure on the $\mathrm{ZrO}_{2}$ deposition process at room temperature. They observed a clear transition from an oxidic sputtering mode (for high oxygen content) to a metallic sputtering mode (for low oxygen content) which resulted into different optical and structural properties of the grown $\mathrm{ZrO}_{2}$ films. In that study and in the other reported deposition studies, $\mathrm{ZrO}_{2}$ thin films were grown on either natively-oxidized or HF-cleaned $\mathrm{Si}(100)$ substrates. But to the best of our knowledge, there are no growth studies at room temperature reported which investigate the initial growth of thin $\mathrm{ZrO}_{2}$ films directly on amorphous $\mathrm{Si}(\mathrm{a}-\mathrm{Si})$. This can be relevant for applications where oxides are deposited on a-Si, such as contact and passivation layers for a-Si solar cells. ${ }^{39,45}$ In the present chapter, we use in vacuo high-sensitivity LEIS (HS-LEIS) to study the initial stages of growth of reactive DC sputtered $\mathrm{ZrO}_{2}$ thin films on a-Si, reactively sputtered sub-stoichiometric $\mathrm{Si}$ nitride (denoted as $\mathrm{SiN}$ ), and $\mathrm{SiO}_{2}$ substrate layers for two gas deposition conditions with high and low oxygen content, representing the two mentioned oxidic and metallic sputtering modes. Apart from a-Si, the other $\mathrm{SiN}$ and $\mathrm{SiO}_{2}$ substrate layers have been selected to study the influence of the Si passivation (by nitridation or oxidation) on the $\mathrm{ZrO}_{2}$ growth. In addition, in vacuo X-ray photoelectron spectroscopy (XPS) is used to determine the $\mathrm{ZrO}_{2}$ stoichiometry and its initial interaction with the underneath a-Si layer for both deposition conditions. Finally, ex-situ angular-resolved XPS (AR-XPS) measurements are performed to investigate the oxygen diffusion response upon thermal oxidation of the $\mathrm{ZrO}_{2} / \mathrm{Si}$ system.

\subsection{Experimental}

$\mathrm{ZrO}_{2}$ layers were prepared at room temperature using reactive DC magnetron sputtering from a metallic $\mathrm{Zr}$ target in an argon-oxygen atmosphere. Two deposition conditions with different oxygen content were chosen for growing the $\mathrm{ZrO}_{2}$ layers. One with high oxygen content and another with low oxygen content, representing both oxidic and metallic sputtering modes observed and described earlier in ref. ${ }^{157}$. For the high 
oxygen conditions, a 1:1 gas mixture of $\mathrm{Ar}: \mathrm{O}_{2}$ was chosen to achieve oxidic sputtering mode. For the low oxygen conditions, a mixture of $0.66 \% \mathrm{O}_{2}$ in Ar was used, which resulted in metallic sputtering mode, following ref. ${ }^{157}$. We note that specific conditions may vary from one deposition set-up to another and only act as a guide. However, the optimization of the conditions was not part of this study. For simplicity, $\mathrm{ZrO}_{2}$ layers deposited with high oxygen content and low oxygen content are referred in the text as high-O $\mathrm{ZrO}_{2}$ and low-O $\mathrm{ZrO}_{2}$, respectively.

High-O and low-O $\mathrm{ZrO}_{2}$ layers with thicknesses ranging from 0 to $4 \mathrm{~nm}$ were deposited on top of $5 \mathrm{~nm}$ a-Si, $1.5 \mathrm{~nm} \mathrm{SiN} / 4 \mathrm{~nm}$ a-Si and $1.5 \mathrm{~nm} \mathrm{SiO} / 4 \mathrm{~nm}$ a-Si layers (see layered models in Fig. 6.1). All layered systems were grown onto natively oxidized super-polished Si substrates in an ultra-high vacuum (UHV) setup with base pressure $<2$ $\mathrm{x} 10^{-10} \mathrm{mbar}$, and their respective layer thicknesses were monitored using quartz mass balances (QMBs), calibrated by ex situ X-ray reflectivity (XRR) using a PANalytical Empyrean $\mathrm{X}$-ray diffractometer $(\mathrm{Cu}-\mathrm{K} \alpha$ radiation, $0.154 \mathrm{~nm})$.

(a)

$\mathrm{X} \mathrm{nm} \mathrm{ZrO}_{2}$
$5.0 \mathrm{~nm}$ a-Si
$\mathrm{Si}(100)$
$X=0$ to $4 \mathrm{~nm}$

(b)

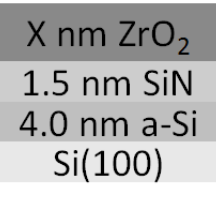

(c)

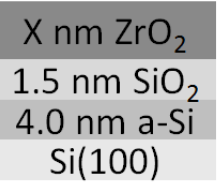

Fig. 6.1. Layered models for $\mathrm{ZrO}_{2}$ on (a) a-Si, (b) $\mathrm{SiN}$ and (c) $\mathrm{SiO}_{2}$.

For $\mathrm{ZrO}_{2}$ thickness calibration, several samples with thicknesses in the range between $\sim 2.0$ and $10 \mathrm{~nm} \mathrm{ZrO}_{2}$ were deposited on top of $5 \mathrm{~nm}$ a-Si layers onto $\mathrm{Si}$ substrates for both deposition conditions. As an example, Fig. 6.2 shows XRR measurements (stars) from $8.9 \mathrm{~nm}$ (a) and $10.0 \mathrm{~nm}$ (b) $\mathrm{ZrO}_{2}$ layers deposited under respectively high-O and low-O conditions, together with $\mathrm{GenX}^{294}$ simulations (solid lines) based on the layered models presented in the insets. The GenX program varied thickness, density and roughness of each of the model layers in order to minimize the differences between model simulations and experimental data. ${ }^{294}$ The accuracy in determining the deposited $\mathrm{ZrO}_{2}$ layer thickness and layer density was approximately \pm 0.2 $\mathrm{nm}$ and $\pm 0.3 \mathrm{~g} \cdot \mathrm{cm}^{-3}$, respectively. The calibration of the $\mathrm{ZrO}_{2}$ thickness was finally obtained from a plot of the extracted as deposited $\mathrm{ZrO}_{2}$ thickness (including the $\mathrm{ZrO}_{2}$ thickness that was used to form $\mathrm{ZrSiO}_{4}$ ) as a function of $\mathrm{Zr}$ QMB thickness for all investigated thicknesses between $\sim 2$ and $10 \mathrm{~nm}$. 


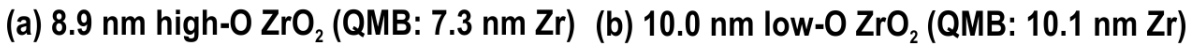
on $5.0 \mathrm{~nm}$ a-Si onto $\mathrm{Si}(100)$

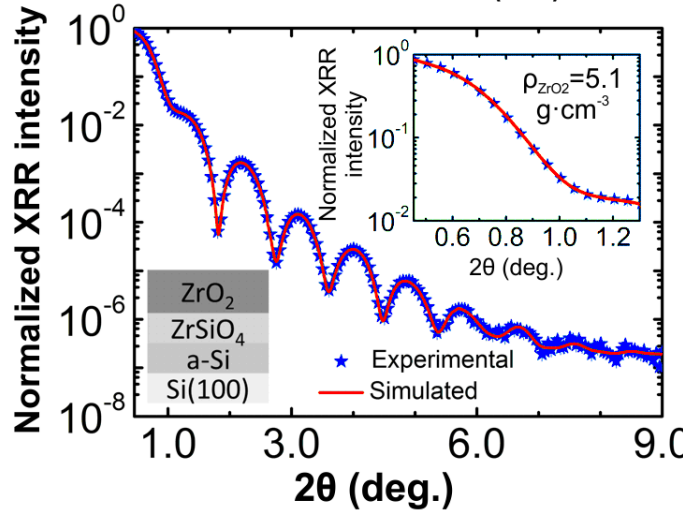
on $5.0 \mathrm{~nm}$ a-Si onto $\mathrm{Si}(100)$

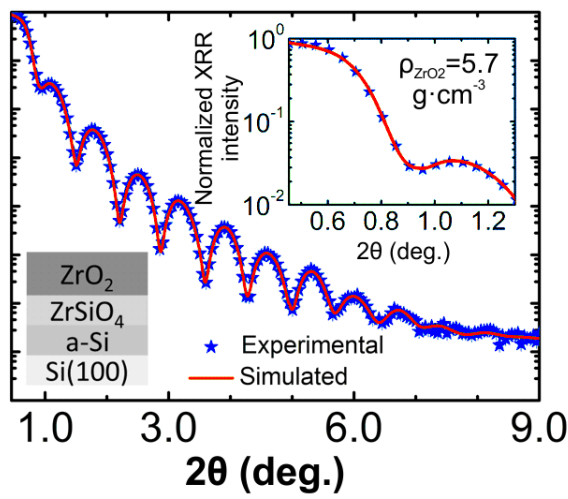

Fig. 6.2. Experimental (stars) and simulated (solid line) specular XRR data for (a) a $8.9 \mathrm{~nm}$ high-O $\mathrm{ZrO}_{2}$ (7.3 nm Zron QMBs) and (b) a $10.0 \mathrm{~nm}$ low-O ZrO 2 (10.1 nm Zron QMBs) samples deposited on $5.0 \mathrm{~nm}$ a-Si onto $\mathrm{Si}(100)$ substrates. Left-bottom (a) and (b) inset shows the layered model used for each simulation. Right-top (a) and (b) inset presents the XRR spectrum in the region close to the critical angle, used to extract the $\mathrm{ZrO}_{2}$ density.

All a-Si, $\mathrm{SiN}$ and $\mathrm{SiO}_{2}$ layers were deposited at room temperature using typical DC or reactive DC magnetron sputtering from a $\mathrm{Si}$ target either in $\mathrm{Ar}$ atmosphere (for a-Si), an $\mathrm{Ar} / \mathrm{N}_{2}(5: 4)$ mixture (for $\mathrm{SiN}$ ), or an $\mathrm{Ar} / \mathrm{O}_{2}(25: 4)$ mixture (for $\mathrm{SiO}_{2}$ ). The sputtering process was performed with a constant cathode power of $\sim 8 \mathrm{~W}$ and a pressure of $\sim 10^{-3}$ mbar. (Detailed conditions can be found in ref. ${ }^{353}$ ).

In vacuo XPS measurements were performed to determine the stoichiometry of the $\mathrm{ZrO}_{2}$ layers, and their initial interaction with the underlying a-Si layer for the high-O and low-O conditions. For that, $10 \mathrm{~nm}$ - and $1 \mathrm{~nm}$-thick $\mathrm{ZrO}_{2}$ layers were respectively deposited on top of $5 \mathrm{~nm}$ a-Si onto Si substrates for both conditions, transferred under vacuum ( $\left.2 \times 10^{-10} \mathrm{mbar}\right)$ to the measurement chamber, and finally analyzed by XPS. The XPS instrument used was a Thermo Theta Probe spectrometer using monochromatic Al$\mathrm{K} \alpha$ radiation $(\mathrm{h} v=1486.6 \mathrm{eV})$. XPS data was evaluated by means of the Thermo Avantage software, ${ }^{358}$ using Shirley backgrounds and Gaussian-Lorentzian fitted peaks. ${ }^{359}$ Scofield sensitivity factors were employed for quantification. ${ }^{360}$

In vacuo HS-LEIS measurements during high-O and low-O $\mathrm{ZrO}_{2}$ growth on a-Si, $\mathrm{SiN}$ and $\mathrm{SiO}_{2}$ substrate layers were carried out using an ION-TOF GmbH Qtac ${ }^{100} \mathrm{HS}$ LEIS spectrometer with a base pressure of $2 \times 10^{-10}$ mbar, for thicknesses up to which $\mathrm{ZrO}_{2}$ formed a closed layer. Detailed information about the setup can be found in ref. ${ }^{276}$. Deposited samples were transferred under vacuum $\left(2 \times 10^{-10} \mathrm{mbar}\right)$ to the LEIS analysis chamber. The work pressure in the LEIS analysis chamber was $\sim 2 \times 10^{-8}$ mbar during all 
measurements, corresponding to $\mathrm{He}$ used in the ion gun. Sample transfer and measurement were carried out within $<10$ min after deposition in order to avoid surface contamination. All LEIS measurements were performed using a $\mathrm{He}^{+}$ion beam with an energy of $3 \mathrm{keV}$ and a beam current of 3-5 nA, measured with a Faraday cup. The average ion dose was $2.2 \times 10^{14} \mathrm{He}^{+}$ions $/ \mathrm{cm}^{2}$, below the "static limit" for $3 \mathrm{keV} \mathrm{He}$ ions which implied that less than $1 \%$ of the surface was sputtered away during the LEIS analysis. ${ }^{339}$

Oxygen diffusion through $\mathrm{ZrO}_{2}$ thin films upon thermal oxidation was investigated by ex situ AR-XPS. For that, $2 \mathrm{~nm}$ low-O $\mathrm{ZrO}_{2}$ was deposited on $5 \mathrm{~nm}$ a-Si onto $\mathrm{Si}(100)$. These low-O conditions for growing $\mathrm{ZrO}_{2}$ were selected due to the lower interaction of $\mathrm{ZrO}_{2}$ with a-Si which provided larger amount of elemental $\mathrm{Si}$ and enabled an easier oxygen detection of the underneath a-Si layer. A thickness of $2 \mathrm{~nm}$ for the $\mathrm{ZrO}_{2}$ film was chosen slightly larger $(\sim 0.3 \mathrm{~nm})$ than the determined closed layer thickness to ensure complete layer closure for these conditions. Several identical $\mathrm{ZrO}_{2}$ thin film samples were annealed during $20 \mathrm{~min}$ at different temperatures (from as deposited to $600^{\circ} \mathrm{C}$ ) under atmospheric environment in a CARBOLITE Elf laboratory furnace that allowed a homogeneous sample heating. Ex-situ AR-XPS measurements were conducted before and after annealing, using the previously mentioned XPS instrument and data analysis. The crystalline structure of both $\mathrm{ZrO}_{2}$ and a-Si films was also investigated. For that, $\mathrm{X}$ ray diffraction (XRD) patterns of the $2 \mathrm{~nm}$ low-O $\mathrm{ZrO}_{2}$ on $5 \mathrm{~nm}$ a-Si samples were recorded using a PANalytical Empyrean X-ray diffractometer $(\mathrm{Cu}-\mathrm{K} \alpha$ radiation, 0.154 $\mathrm{nm})$. In addition, surface morphologies of the as deposited and annealed samples were analyzed using an ex situ BRUKER Dimension Edge atomic force microscope (AFM) equipped with a $\mu$ masch Hi'Res-C14 tip, featuring a diamond-like spike (radius $<1 \mathrm{~nm}$ ) on the apex of a Si tip, making it suitable for high-resolution scans of flat samples. The AFM image analysis (morphology, roughness) was performed using the WSxM software package. $^{306}$

\subsection{Results and discussion}

\subsection{1. $\mathrm{ZrO}_{2}$ growth on a-Si}

Since under-stoichiometric oxides may allow $\mathrm{O}$ diffusion through their structure in contrast to stoichiometric oxides, ${ }^{361}$ the $\mathrm{ZrO}_{2}$ stoichiometry of the produced layers for high-O and low-O conditions is investigated first. For that, $10 \mathrm{~nm}$-thick $\mathrm{ZrO}_{2}$ layers are deposited for both conditions on $5 \mathrm{~nm}$ a-Si onto Si substrates, and in vacuo analyzed by XPS (see Zr-3d XPS spectra in Fig. 6.3). Note that the thickness of the $\mathrm{ZrO}_{2}$ layer is selected to ensure that only this layer is investigated, since XPS averages the atomic composition and the chemical state of the atoms over the outer 10 to 20 atomic layers, ${ }^{276}$ corresponding to a depth of $\sim 3$ to $6 \mathrm{~nm}$. 
(a) High-O ZrO

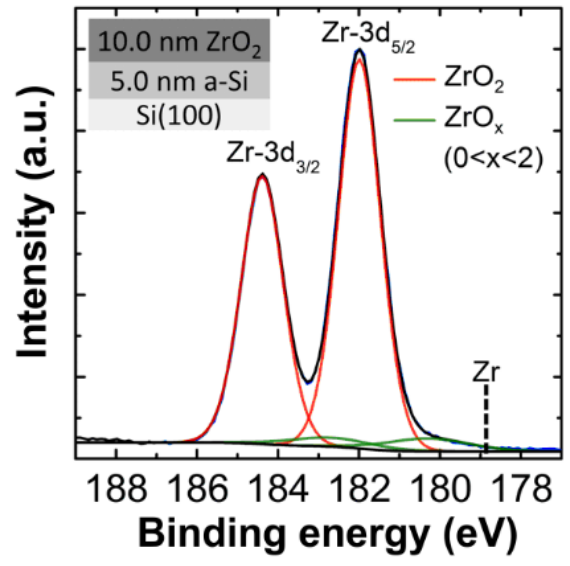

(b) Low-O ZrO

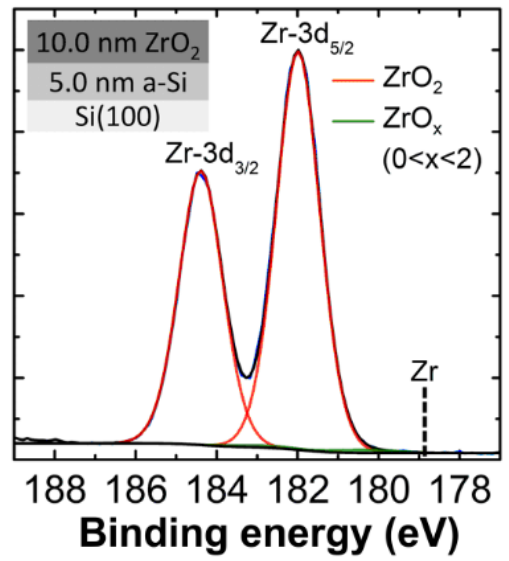

Fig. 6.3. $\mathrm{Zr}-3 \mathrm{~d} X P S$ spectra for high-O $\mathrm{ZrO}_{2}$ (a) and low-O $\mathrm{ZrO}_{2}$ (b). $\mathrm{Zr}-3 \mathrm{~d}_{3 / 2}$ (left) and $\mathrm{Zr}-3 \mathrm{~d}_{5 / 2}$ (right) peaks which correspond to $\mathrm{ZrO}_{2}$ (red solid lines) and $\mathrm{ZrO}_{x}$ (green solid lines), and the layered models of the deposited structures (insets) are displayed in (a) and (b). Note that no elemental $\mathrm{Zr}$ is detected for both deposition conditions, as pointed by the dashed lines.

Most of zirconium is oxidized, forming $\mathrm{ZrO}_{2}$ and no elemental $\mathrm{Zr}$ is detected for both $\mathrm{ZrO}_{2}$ deposition conditions, as depicted in Fig. 6.3. There is a small amount of $\mathrm{ZrO}_{\mathrm{x}}$ $(\sim 3 \%)$ for the high-O conditions which is almost not present for low-O conditions. This may be attributed to the fact that for high-O conditions the $\mathrm{Zr}$ target is not yet saturated by $\mathrm{O}_{2}$ in the $\mathrm{Ar}+\mathrm{O}_{2}$ mixture, since initially the $\mathrm{Zr}$ target is only pre-sputtered in $\mathrm{Ar}$, and $\mathrm{O}_{2}$ is introduced just before ( $\left.<1 \mathrm{~min}\right)$ the deposition starts. Instead for low-O conditions, the $\mathrm{Zr}$ target is already saturated at the beginning of the deposition due to an additional pre-sputtering step of $\sim 5 \mathrm{~min}$ in $\mathrm{Ar}+\mathrm{O}_{2}$ mixture. The different approach in the case of high-O conditions is chosen to limit the influence of oxidation of the a-Si substrate layer and to ensure sufficient cleaning of the $\mathrm{Zr}$ target, in view of the lower sputter rate for the oxidic sputtering mode. The stoichiometry of the $10 \mathrm{~nm}$-thick $\mathrm{ZrO}_{2}$ layers, as determined from the $\mathrm{O}-1 \mathrm{~s}$ and $\mathrm{Zr}-3 \mathrm{~d}$ peak areas, resulted in stoichiometric ratios $(\mathrm{O} / \mathrm{Zr})$ of $1.98 \pm 0.03$ and $1.99 \pm 0.03$ for high-O and low-O conditions, respectively, close to bulk $\mathrm{ZrO}_{2}$. Note that similar $\mathrm{ZrO}_{2}$ deposition conditions with high and low oxygen content showed different stoichiometric behavior in ref.(Venkarataj et al. ${ }^{157}$ ). There, the $\mathrm{ZrO}_{2}$ layers produced with low oxygen content (denoted as metallic sputtering mode) were understoichiometric and the $\mathrm{ZrO}_{2}$ layers produced with high oxygen content (denoted as oxidic sputtering mode) were stoichiometric. A very sharp transition was observed between both metallic and oxidic sputtering modes, ${ }^{157}$ and it is possible that our low-O $\mathrm{ZrO}_{2}$ deposition conditions are just in that transition region. 
To confirm that low- $\mathrm{O} \mathrm{ZrO}_{2}$ conditions are in the transition region, we compare the mass density of our $10 \mathrm{~nm}$-thick $\mathrm{ZrO}_{2}$ layers grown by our two deposition conditions to the average mass density of the $\mathrm{ZrO}_{2}$ layers produced by the two modes observed in ref. ${ }^{157}$. Mass densities of our layers are determined by XRR using the layered models depicted in Fig. 6.2, according to the procedure described in Sec. 6.2. The high sensitivity of the XRR analysis to the $\mathrm{ZrO}_{2}$ density comes from the critical angle, which is related to the average electron (proportional to mass) density of the layered structure, as well as the amplitude of the oscillations, which is related to the optical contrast between $\mathrm{ZrO}_{2}$ and the Si substrate, which also strongly depends on the mass density of the $\mathrm{ZrO}_{2}$ layer. Mass density values of $5.1 \pm 0.3$ and $5.7 \pm 0.3 \mathrm{~g} \cdot \mathrm{cm}^{-3}$ are obtained for our high-O and low $\mathrm{O} \mathrm{ZrO}_{2}$ layers (see Figs. 6.2(a) and 6.2(b), respectively).

In the study carried out in ref. ${ }^{157}$, average mass density values of 6.5 and $5.2 \mathrm{~g} \cdot \mathrm{cm}^{-}$ ${ }^{3}$ were determined for the $\mathrm{ZrO}_{2}$ layers deposited by the metallic and the oxidic sputtering modes, respectively. The density of the high-O $\left(5.1 \pm 0.3 \mathrm{~g} \cdot \mathrm{cm}^{-3}\right)$ and the oxidic $\mathrm{ZrO}_{2}$ layers $\left(5.2 \mathrm{~g} \cdot \mathrm{cm}^{-3}\right)$ is similar, denoting comparable conditions. Contrarily, the density of the low-O $\mathrm{ZrO}_{2}$ layers $\left(5.7 \pm 0.3 \mathrm{~g} \cdot \mathrm{cm}^{-3}\right)$ is lower $(\sim 12 \%)$ than the density of the metallic $\mathrm{ZrO}_{2}$ layers $\left(6.5 \pm 0.3 \mathrm{~g} \cdot \mathrm{cm}^{-3}\right)$. Specifically, the low-O $\mathrm{ZrO}_{2}$ density is between the metallic mode density, close to bulk $\operatorname{Zr}\left(6.52 \mathrm{~g} \cdot \mathrm{cm}^{-3}\right)^{38}$, and the oxidic mode density $(5.2$ $\left.\mathrm{g} \cdot \mathrm{cm}^{-3}\right){ }^{157}$ and exhibits a value close to bulk $\mathrm{ZrO}_{2}\left(5.68 \mathrm{~g} \cdot \mathrm{cm}^{-3}\right){ }^{38}$ This confirms our suggestion that low-O $\mathrm{ZrO}_{2}$ deposition conditions are just in the transition region between the metallic and the oxidic sputtering modes, representing a semi-metallic sputtering mode. In addition, based on the previous analysis, high-O $\mathrm{ZrO}_{2}$ deposition conditions correspond to an oxidic sputtering mode.

Apart from the stoichiometry, it is also interesting to study the initial gas vaporsolid reaction between the sputtered $\mathrm{ZrO}_{2}$ and the underlying a-Si layer for high-O and low-O deposition conditions. For that, $1 \mathrm{~nm}$-thick $\mathrm{ZrO}_{2}$ layers are grown on $5 \mathrm{~nm}$ a-Si, and also measured by in vacuo XPS (see Si-2p XPS spectra for both conditions in Fig. 6.4). Note that the $\mathrm{ZrO}_{2}$ thickness is selected in this case to study the full $\mathrm{ZrO}_{2} / \mathrm{Si}$ interface (including both $\mathrm{ZrO}_{2}$ and a-Si layers), since the maximum XPS information depth is about $6 \mathrm{~nm} .^{276}$

$\mathrm{ZrO}_{2}$ deposition produces a clear peak at about $102 \mathrm{eV}$ corresponding to silicate $\left(\mathrm{ZrSiO}_{4}\right)$ formation, and no clear stoichiometric $\mathrm{SiO}_{2}$ peak is observed $(\sim 103.5 \mathrm{eV})$ for both $\mathrm{ZrO}_{2}$ growth conditions (see Si-2p XPS peaks in Fig. 6.4). This is in line with other studies where only $\mathrm{ZrSiO}_{4}$ formation, rather than $\mathrm{SiO}_{2}$, was observed upon initial $\mathrm{ZrO}_{2}$ deposition on $\mathrm{SiO}_{2} / \mathrm{Si}$ substrates. ${ }^{362,363}$ It should be noted that before $\mathrm{ZrO}_{2}$ deposition, our $5 \mathrm{~nm}$ a-Si layers are slightly oxidized due to pre-sputtering of the $\mathrm{Zr}$ target in $\mathrm{Ar}+\mathrm{O}_{2}$ atmosphere, even though the target shutter is closed during this period. This means that our $\mathrm{ZrO}_{2}$ deposition process is performed on an oxidized $\mathrm{Si}$ surface with a similar 
behavior as the mentioned studies on $\mathrm{SiO}_{2} / \mathrm{Si}$ substrates. From the potential chemical reactions at the $\mathrm{ZrO}_{2} / \mathrm{Si}$ interface, the ones leading to $\mathrm{ZrSiO}_{4}$ are thermodynamically more favorable (at least by a factor of 10) than the ones leading to $\mathrm{SiO}_{2}{ }^{362}$ Thus, $\mathrm{ZrSiO}_{4}$ formation would be preferred over $\mathrm{SiO}_{2}$ formation. Although some of the potential reactions also show $\mathrm{ZrSi}_{\mathrm{x}}$ products, ${ }^{362}$ the presence of oxygen would reduce their formation, favoring oxidized species such as $\mathrm{ZrSiO}_{4}$.

For high-O $\mathrm{ZrO}_{2}$ conditions, most of $\mathrm{ZrO}_{2}$ interacts with the underneath a-Si, forming $\mathrm{ZrSiO}_{4}$ (see the large $\mathrm{ZrSiO}_{4}$ peak compared to the elemental Si peak in Fig. 6.4(a)). This strong interaction may go beyond the silicate and some sub-stoichiometric $\mathrm{Si}$ oxide $\left(\mathrm{SiO}_{\mathrm{x}}\right)$ may be formed, as shown by the small shift $(\sim 0.3 \mathrm{eV})$ towards higher energies of the $\mathrm{ZrSiO}_{4}$ peak in Fig. 6.4(a). This shift can also be associated to the formation of a silicate rich in $\mathrm{SiO}_{2}$, as observed by XPS in pseudobinary $\left(\mathrm{ZrO}_{2}\right)_{x}\left(\mathrm{SiO}_{2}\right)_{1}$ $x$ alloys with increased $\mathrm{SiO}_{2}$ content. ${ }^{364}$ In contrast for low-O $\mathrm{ZrO}_{2}$ conditions, only a small amount of a-Si $(22 \%)$ reacts with $\mathrm{ZrO}_{2}$ to form some interfacial $\mathrm{ZrSiO}_{4}$, and no apparent $\mathrm{SiO}_{\mathrm{x}}$ or $\mathrm{SiO}_{2}$-rich silicate is detected (see the small $\mathrm{ZrSiO}_{4}$ peak appearing at 102.0 $\pm 0.1 \mathrm{eV}$ in Fig. 6.4(b)). The large difference between high-O and low-O conditions can be attributed to the different amount of energetic oxygen particles impacting on the sample, resulting in more intermixing (silicate formation) for the high-O conditions.

(a) High-O ZrO

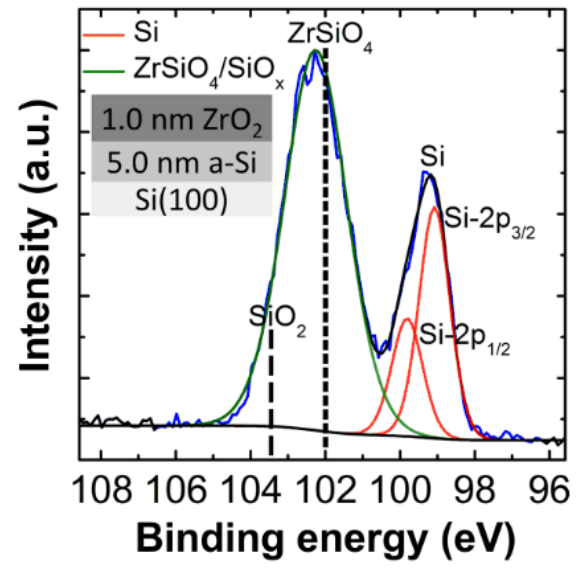

(b) Low-O ZrO

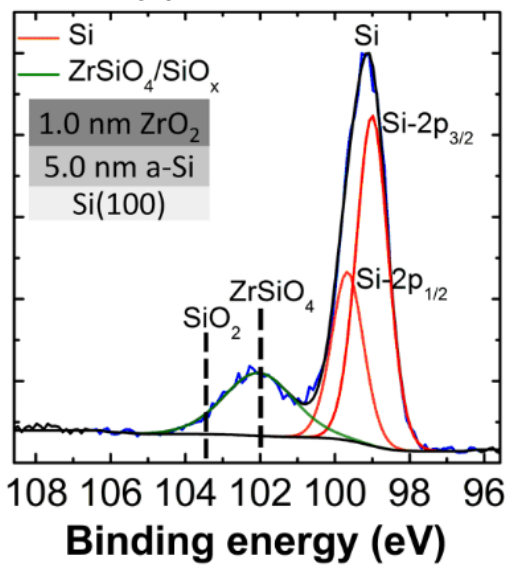

Fig. 6.4. Si-2p XPS spectra for high-O $\mathrm{ZrO}_{2}$ (a) and low-O $\mathrm{ZrO}_{2}$ (b). Elemental $\mathrm{Si}$ is fitted with a doublet (right, red peaks), while $\mathrm{ZrSiO}_{4}$ and/or $\mathrm{SiO}_{\mathrm{x}}$ species are fitted with a single peak for simplicity (left, green peaks). Layered models of the deposited structures are shown in insets. Note that no $\mathrm{SiO}_{2}$ peaks are detected, especially for the low- $\mathrm{O} \mathrm{ZrO}_{2}$ conditions, where only $\mathrm{ZrSiO}_{4}$ is observed, as pointed by the dashed lines. 
We now study the initial growth of $\mathrm{ZrO}_{2}$ on a-Si, including the determination of the thickness when the $\mathrm{ZrO}_{2}$ layer closes for both conditions. We define a layer of one or more elements to be closed when its surface only contains these elements and other elements from the underneath layer are no longer present on this surface. Our LEIS tool exhibits a high sensitivity to the surface elements which enables a compositional analysis of the outermost atomic layer. This composition can be determined by the surface peaks of the elements present on the surface. ${ }^{270}$

LEIS spectra of the deposited $\mathrm{ZrO}_{2}$ layers on a-Si for high-O and low-O conditions are presented in Figs. 6.5(a) and 6.5(b), respectively. For both conditions, the $\mathrm{Zr}$ and $\mathrm{O}$ surface peaks increase, while $\mathrm{Si}$ decreases during $\mathrm{ZrO}_{2}$ growth (Figs. 6.5(a) and 6.5(b)). For high-O conditions, the $\mathrm{Si}$ surface peak vanishes at about $3.4 \mathrm{~nm} \mathrm{ZrO}_{2}$, just when the $\mathrm{Zr}$ surface peak saturates (see Fig. 6.5(a)). This means that about $3.4 \mathrm{~nm} \mathrm{ZrO}_{2}$ are needed to close the $\mathrm{ZrO}_{2}$ layer on a-Si. This large amount of material required for forming a closed layer can be related to stronger intermixing by $\mathrm{ZrSiO}_{4}$ and/or $\mathrm{SiO}_{\mathrm{x}}$ formation for this conditions, as also observed by XPS (see Fig. 6.4). In contrast, for low-O conditions, the Si surface peak fully disappears at about $1.7 \mathrm{~nm} \mathrm{ZrO}_{2}$, at the point when the $\mathrm{Zr}$ surface peak reaches saturation (Fig. 6.5(b)). Due to the minor interfacial silicate produced, the $\mathrm{ZrO}_{2}$ layer closes at half of the thickness for this conditions.

For high-O conditions, the $\mathrm{O}$ surface peak quickly saturates $\left(\sim 0.3 \mathrm{~nm} \mathrm{ZrO}_{2}\right)$ and stays constant due to the strong initial oxidation of the full Si surface and immediate $\mathrm{ZrSiO}_{4}$ formation just when the target shutter opens (see Fig. 6.5(a)). Instead for low-O conditions, the $\mathrm{O}$ surface peak slowly increases and at the end saturates during $\mathrm{ZrO}_{2}$ deposition due to less amount of oxygen energetic particles present, which initially leads to partial surface oxidation and sub-stoichiometric silicate formation (see Fig. 6.5(b)). This initial difference in surface oxidation also results in a different intensity of the $\mathrm{Si}$ sub-surface signal (low energy "tail" to the Si surface peak), which arises due to reionization of $\mathrm{He}^{0}$ that backscatters on $\mathrm{Si}$ atoms below the surface. ${ }^{270}$ For high-O conditions, the oxygen surface concentration is directly saturated for $0.3 \mathrm{~nm}$ deposited $\mathrm{ZrO}_{2}$ (Fig. 6.5(a)). The presence of electronegative $\mathrm{O}$ species results in an enhanced reionization probability, thereby increasing the tail signal of Si compared to the bare a$\mathrm{Si}$ substrate layer. Since for low-O conditions the surface concentration of oxygen only saturates gradually with increasing $\mathrm{ZrO}_{2}$ thickness, the height of the $\mathrm{Si}$ sub-surface signal increases up to about $1 \mathrm{~nm}$ deposited $\mathrm{ZrO}_{2}$ (Fig. 6.5(b)). 
(a) High-O ZrO

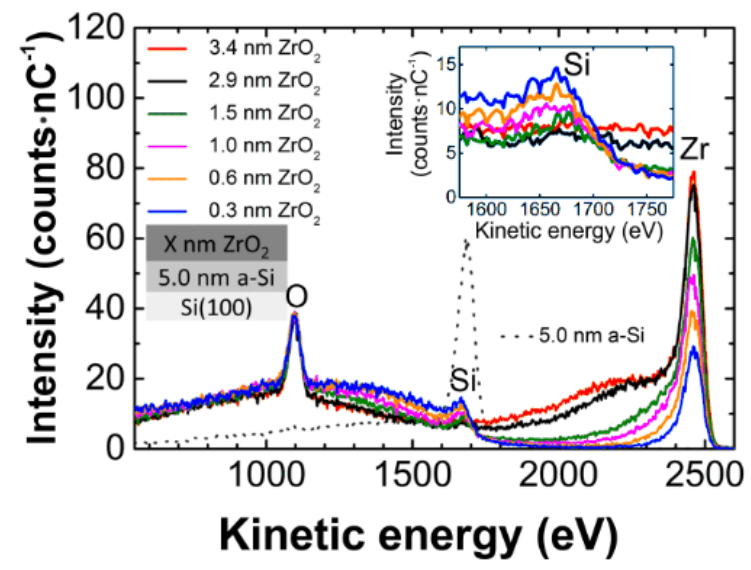

(b) Low-O ZrO

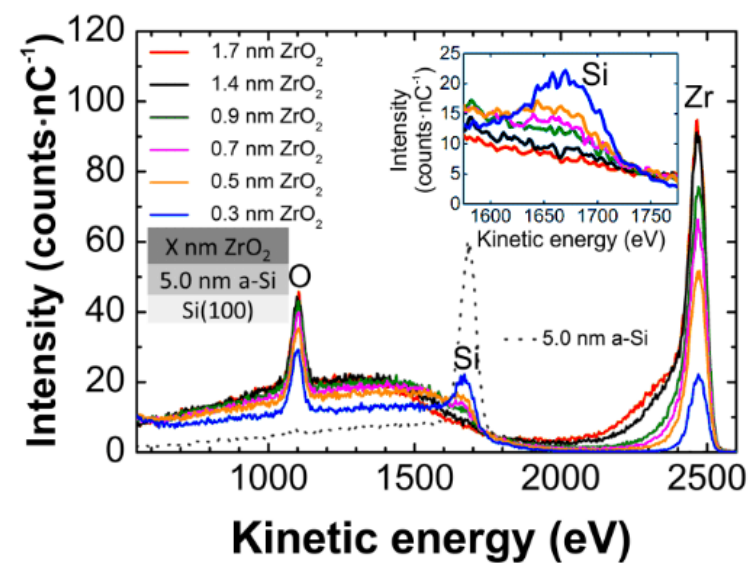

Fig. 6.5. LEIS spectra of 0.3 to $3.4 \mathrm{~nm}$ high-O (a) and 0.3 to $1.7 \mathrm{~nm}$ low-O (b) $\mathrm{ZrO}_{2}$ layers grown on $5 \mathrm{~nm}$ a-Si onto $\mathrm{Si}(100)$ substrate (solid lines). The magnified view of the Si peak and the layered model of the deposited structure are presented in the respective right-top and left-bottom insets in (a) and (b). $5 \mathrm{~nm}$ a-Si spectra are displayed by dashed lines in (a) and (b).

It should be noted here that LEIS cannot discriminate between intermixing by $\mathrm{ZrSiO}_{4}$ formation and 3D island growth. Both intermixing and 3D island formation would give similar LEIS spectra, leading to a wrong interpretation of our results. Hence, surface morphologies of our grown samples on a-Si for several $\mathrm{ZrO}_{2}$ thicknesses and for both deposition conditions are investigated by AFM. As an example, AFM images of samples with intermediate thicknesses to close the $\mathrm{ZrO}_{2}$ layer on a-Si of $1.0 \mathrm{~nm}$ (for highO) and $0.9 \mathrm{~nm}$ (for low-O) conditions are presented in Figs. 6.6(a) and 6.6(b), respectively. Both samples are flat (see Fig. 6.6), without observed apparent surface morphology changes relative to the initial $\mathrm{Si}$ wafer. The high-O $\mathrm{ZrO}_{2}$ sample exhibits a 
root mean square (RMS) roughness value of $0.18 \pm 0.05$, and a value of $0.17 \pm 0.05 \mathrm{~nm}$ is obtained for the low-O $\mathrm{ZrO}_{2}$ sample. These values and the ones obtained for all other thicknesses are similar to the bare $\mathrm{Si}(100)$ substrate, which exhibits an average RMS roughness of $0.20 \pm 0.05 \mathrm{~nm}$. As a result, we can exclude the formation of 3D islands as decisive process for layer closure in $\mathrm{ZrO}_{2}$ growth, which seems dominated by intermixing.

(a) High-O ZrO

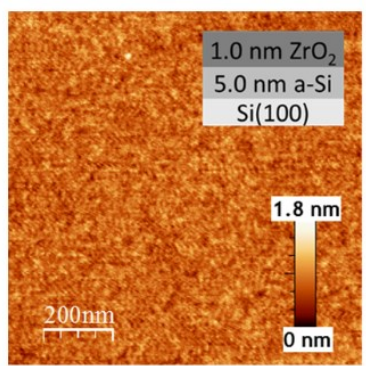

(b) Low-O ZrO

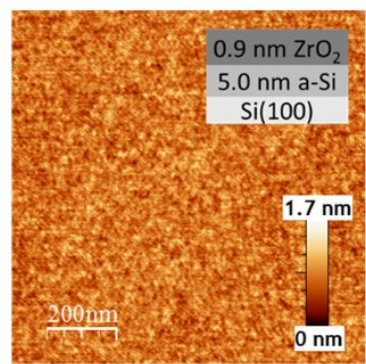

Fig. 6.6. AFM images $(1 \mathrm{x} 1 \mu \mathrm{m})$ of samples with intermediate thickness of $\mathrm{ZrO}_{2}$ (no closed layer) on a-Si for high-O (a) and low-O (b) conditions. Layered models of the deposited structures are shown in insets.

The intensities of the $\mathrm{Zr}, \mathrm{Si}$ and $\mathrm{O}$ surface peaks presented in Fig. 6.5 are proportional to the surface concentration (or coverage) of the corresponding element. ${ }^{276}$ In the determination of such surface coverage, we use the surface peak area, which provides more accurate results for the following quantitative surface composition analysis. ${ }^{270}$ The reference peak areas for $\mathrm{Zr}$ and $\mathrm{Si}$ are directly obtained from $10 \mathrm{~nm}$ thick $\mathrm{Zr}$ (free of oxygen) and a-Si deposited samples in vacuo measured by LEIS. The obtained reference values for $\mathrm{Zr}$ and $\mathrm{Si}$ are $(18.2 \pm 0.9) \times 10^{3}$ and $(4.0 \pm 0.1) \times 10^{3}$ counts $\cdot \mathrm{nC}^{-1}$, respectively. Since pure references for $\mathrm{O}$ do not exist in literature due to the experimental difficulties of directly measuring solid oxygen, an indirect method to obtain this $\mathrm{O}$ reference peak area will be discussed later. The surface atomic density $N_{i}^{\text {ref }}$ of an element $i$ in a reference sample (compound or element) with known mass density $\rho$, can be estimated by

$$
N_{i}^{r e f} \approx\left(\frac{n_{i} \rho N_{A V}}{M}\right)^{2 / 3},
$$

where $n_{i}$ is the stoichiometric number of the element $i$ in the compound ( $n_{i}=1$ for a single element), $M$ is the molar mass of the compound (or element), and $N_{A V}$ is Avogadro's number. ${ }^{341}$ Mass density values of $5.1 \pm 0.3,5.7 \pm 0.3$ and $2.3 \pm 0.3 \mathrm{~g} \cdot \mathrm{cm}^{-3}$ are determined from the respective fits to the XRR data of 10 nm-thick high-O $\mathrm{ZrO}_{2}$, low-O $\mathrm{ZrO}_{2}$ and aSi deposited samples, following the method described in Sec. 6.2. The surface atomic 
densities $N_{i}^{r e f}$ of $\mathrm{Zr}$, Si and $\mathrm{O}$ atoms in either high-O $\mathrm{ZrO}_{2}$, low-O $\mathrm{ZrO}_{2}$ or a-Si samples are then calculated using the obtained mass density values $\rho$ according to Eq. 1 . The surface coverage $C_{i}^{\text {surf }}$ for an element $i$, expressed as surface atomic fraction, can be determined as ${ }^{270}$

$$
C_{i}^{\text {surf }}=\left[\sum_{j=1}^{j_{\max }} \frac{S_{j} S_{i}^{r e f} N_{j}^{r e f}}{S_{i} S_{j}^{r e f} N_{i}^{r e f}}\right]^{-1},
$$

where $S_{j}$ are the surface peak areas, $S^{r e f}$ are the reference peak areas, and $N_{j}^{r e f}$ are the surface atomic densities for the different surface elements (from $j=1$ to $j_{\max }$ ). Using Eq. 2, all surface coverages $\left(\mathrm{Zr}, \mathrm{Si}\right.$ and $\mathrm{O}$ ) for high-O and low-O $\mathrm{ZrO}_{2}$ layers grown on a-Si are finally obtained, and are displayed in Fig. 6.7(a) and Fig. 6.7(b), respectively. It should be noted that this equation can only be applied under absence of matrix effects. ${ }^{270}$ If there are no matrix effects, when the Zr LEIS signal is plotted against the Si LEIS signal for different amounts of deposited $\mathrm{ZrO}_{2}$ on $\mathrm{Si}$, a linear relationship should follow. This has already been observed for the $\mathrm{ZrO}_{2} / \mathrm{Si}$ system, ${ }^{186}$ and was confirmed for the $\mathrm{Zr}$ vs. Si peak area in this work (not shown). Thus, matrix effects seem not to be present for our system, and Eq. 2 can successfully be applied. However, to apply Eq. 2 for obtaining the surface coverages, the $\mathrm{O}$ reference peak area is still required. To obtain this reference, we know that for each deposited amount of $\mathrm{ZrO}_{2}$, the surface Vegard's law must be fulfilled ${ }^{340}$

$$
\sum_{j=1}^{j_{\max }} C_{j}^{\text {surf }}=1
$$

First, we assume an initial value for the $\mathrm{O}$ reference peak area. Then, we use this value to calculate all surface coverages by means of Eq. 2 for each deposited amount of $\mathrm{ZrO}_{2}$. This value is changed recursively until Eq. 3 is valid for all deposited thicknesses. The obtained $\mathrm{O}$ reference peak area for both $\mathrm{ZrO}_{2}$ conditions is $1170 \pm 70$ counts $\cdot \mathrm{nC}^{-1}$, similar as the $1180 \pm 83$ counts $\cdot \mathrm{nC}^{-1}$ reference value reported for $\mathrm{O}$ in $\mathrm{SiO}_{2},{ }^{353}$ demonstrating the reliability of this method.

A similar trend in surface $\mathrm{Zr}$ coverage increase with increasing deposited amount of $\mathrm{ZrO}_{2}$ is observed for high-O and low-O $\mathrm{ZrO}_{2}$ conditions, as depicted by squares in Figs. 6.7(a) and 6.7(b), respectively. Although for low-O conditions, the layer closes at half of the thickness required for high-O conditions due to less intermixing, this similar trend denotes that a similar error function like $\mathrm{Zr}$ depth profile develops with increasing $\mathrm{ZrO}_{2}$ thickness for both conditions, though with different interface width, as explained in more detail in ref. ${ }^{353}$. In contrast, there is a faster decrease of Si coverage that goes in parallel with a quicker saturation of the $\mathrm{O}$ coverage for high-O conditions when compared to low-O conditions, as represented by respective triangles and circles in Figs. 
6.7(a) and 6.7(b). This means that for high-O conditions there is a strong initial oxidation which leads to instant $\mathrm{ZrSiO}_{4}$ formation just when the deposition starts, as shown by the saturated $\mathrm{O}$ coverage at $0.2 \mathrm{~nm} \mathrm{ZrO}_{2}$ (Fig. 6.7(a), circles). Instead, for low-O conditions there are not enough $\mathrm{O}$ energetic particles at these initial stages to produce stoichiometric $\mathrm{ZrSiO}_{4}$. Thus, an under-stoichiometric oxygen-poor silicate is formed at the beginning. And only after $0.7 \mathrm{~nm} \mathrm{ZrO}_{2}$, the $\mathrm{O}$ coverage saturates and fully stoichiometric $\mathrm{ZrSiO}_{4}$ can finally be achieved (Fig. 6.7(b), circles).

(a) High-O ZrO

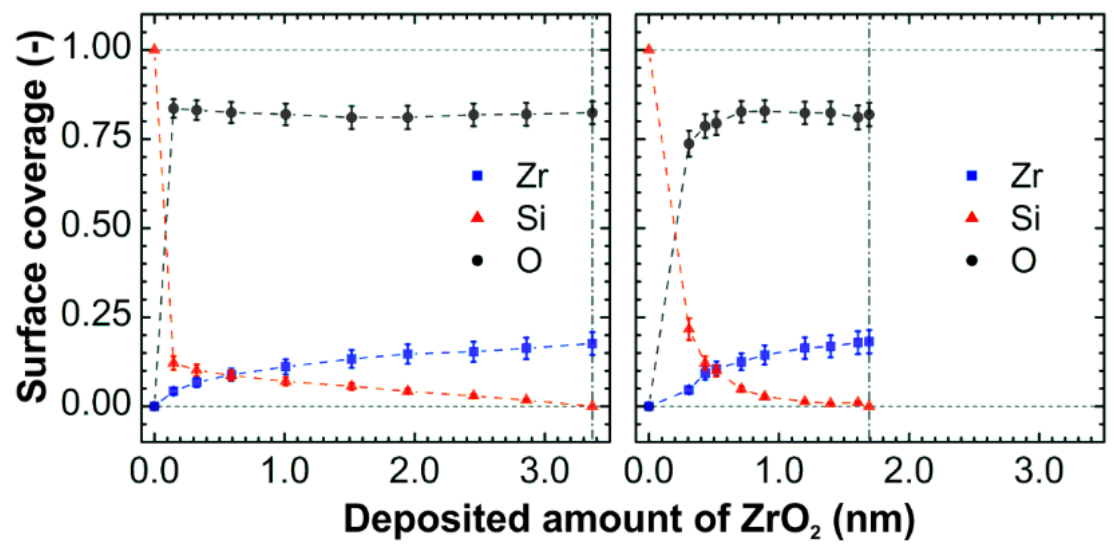

Fig. 6.7. $\mathrm{Zr}$ (squares), $\mathrm{Si}$ (triangles) and $\mathrm{O}$ (circles) surface coverages for high-O (a) and low-O (b) $\mathrm{ZrO}_{2}$ layers grown on $5 \mathrm{~nm}$ a-Si on $\mathrm{Si}(100)$ substrate. The dashed lines through the data points are guides for the eye. The two horizontal dashed lines and the vertical dashed-dot line in (a) and (b) represent the respective limits for the surface coverage (from 0 to 1 ) and the thickness to close the $\mathrm{ZrO}_{2}$ layer on a-Si for each conditions.

It is known that Si surface segregation can influence the growth causing additional intermixing, as observed by several systems deposited by room-temperature magnetron sputtering. ${ }^{349,353}$ Apart from silicate and/or oxide formation, we need to study the Si surface segregation effect in order to check its contribution to the overall intermixing process during $\mathrm{ZrO}_{2}$ growth for both deposition conditions. We can qualitatively study this effect by looking at the in-depth Zr signal ("tail" to the Zr peak) in the LEIS spectra while comparing to the Si surface signal. If there is Si surface segregation, the in-depth $\mathrm{Zr}$ signal just below the surface will appear already saturated for a $\mathrm{ZrO}_{2}$ deposited thickness below the closing of the layer, while the Si surface peak is still visible. Fig. 6.8 shows the LEIS spectra of high-O (a) and low-O (b) $\mathrm{ZrO}_{2}$ layers on a-Si for different thicknesses near to the closing of the layer, in order to compare in-depth $\mathrm{Zr}$ and surface Si signals. 
(a) High-O ZrO

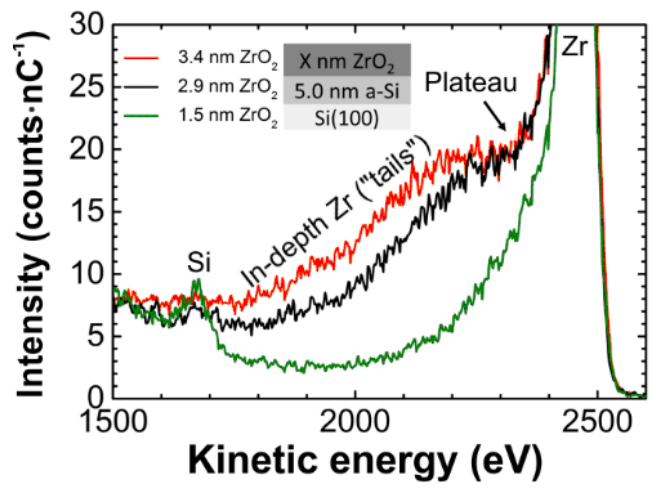

(b) Low-O ZrO

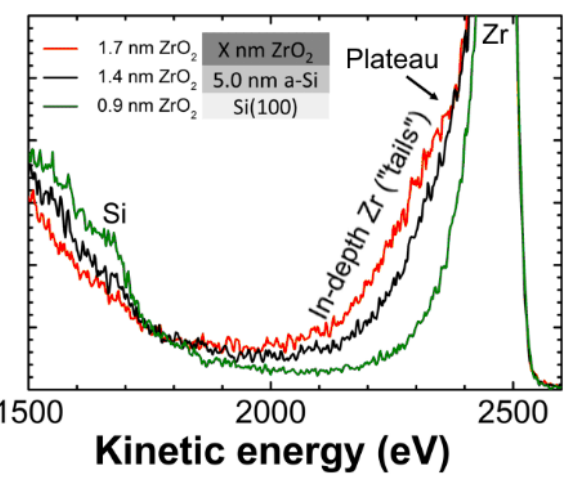

Fig. 6.8. LEIS spectra of high-O (a) and low-O (b) $\mathrm{ZrO}_{2}$ on a-Si for different thicknesses near to the closing of the layer. Top-middle insets in (a) and (b) represent the layered models of the deposited structures. Note that the saturation level of the in-depth $\mathrm{Zr}$ ("tails") signal is achieved by the formation of a plateau close to the $\mathrm{Zr}$ surface peak.

For high-O conditions, the in-depth $\mathrm{Zr}$ signal reaches the saturation level at about $2.9 \mathrm{~nm} \mathrm{ZrO}_{2}$ thickness by the formation of a plateau close to the $\mathrm{Zr}$ peak in the LEIS spectrum while there is still a Si surface peak (Fig. 6.8(a), black spectrum). This means that there is surface segregation of $\mathrm{Si}$ for this conditions and suggests that around $2.9 \mathrm{~nm}$ the grown $\mathrm{ZrO}_{2}$ layer is almost a bulk closed layer. At this point, the Si surface coverage is $2.9 \pm 0.5 \%$ (from Fig. 6.7 (a), triangles), which can be mostly attributed to the amount of $\mathrm{Si}$ segregated towards the surface from the $\mathrm{ZrO}_{2} / \mathrm{Si}$ interface. The formed plateau remains for $\mathrm{ZrO}_{2}$ thicknesses larger than $2.9 \mathrm{~nm}$ while the $\mathrm{Si}$ segregation effect reduces with increasing $\mathrm{ZrO}_{2}$ thickness and disappears totally when the layer closes at about 3.4 $\mathrm{nm} \mathrm{ZrO}_{2}$, as observed by the decrease and the vanishing of the Si surface peak in Fig. 6.8(a). The Si segregation effect detected for this high-O $\mathrm{ZrO}_{2}$ deposition conditions may be related to the large amount of $\mathrm{O}$ energetic particles impacting the sample that can displace and enhance the diffusivity of interfacial $\mathrm{Si}$ atoms. And although $\mathrm{ZrSiO}_{4}$ formation is the most favorable process occurring at the $\mathrm{ZrO}_{2} / \mathrm{Si}$ interface, ${ }^{362}$ a small amount of unbound $\mathrm{Si}$ atoms from this interface can segregate upwards and form surface silicate. This process might be motivated by the minimization of the surface free energy by having $\mathrm{Si}$ on the top rather than $\mathrm{Zr}{ }^{350}$

For low-O conditions, there is no evidence of $\mathrm{Si}$ surface segregation since the indepth Zr ("tails") signal saturates just at the moment when the Si surface peak completely disappears at about $1.7 \mathrm{~nm}$ and the $\mathrm{ZrO}_{2}$ layer closes (Fig. 6.8(b), red spectrum). The absence of Si surface segregation for this low-O conditions might be due to the small amount of $\mathrm{O}$ energetic species impinging the sample which only allow interfacial silicate formation during growth. And as soon as this interfacial zone arrives to its end, the layer loses without surface segregation effects which could lead to additional intermixing. 


\subsubsection{Effect of $\mathrm{Si}$ passivation on $\mathrm{ZrO}_{2}$ growth}

We now study the passivation effect of the a-Si layer (by nitridation or oxidation) on the initial growth of $\mathrm{ZrO}_{2}$ for the two high-O and low-O deposition conditions described in Sec. 6.3.1. To study that, $\mathrm{ZrO}_{2}$ thin films have been grown onto SiN and $\mathrm{SiO}_{2}$ substrate layers, and in vacuo analyzed by LEIS (see layered models of the deposited structures in Figs. 6.1(b), and 6.1(c), respectively). The thickness to close the $\mathrm{ZrO}_{2}$ layer has also been determined for both substrate layers and both deposition conditions. All surface coverages $(\mathrm{Zr}, \mathrm{Si}, \mathrm{O}, \mathrm{N})$ have been extracted from the surface peak areas according to Eq. 2, following a similar procedure as the one discussed in Sec. 6.3.1 for $\mathrm{ZrO}_{2}$ on a-Si, with minor modifications for the growth on $\mathrm{SiN}$ and on $\mathrm{SiO}_{2}$ layers. For $\mathrm{ZrO}_{2}$ on $\mathrm{SiN}$, the used surface atomic densities for $\mathrm{Si}$ and $\mathrm{N}$ atoms are calculated by Eq. 1, using a mass density of $3.6 \pm 0.3 \mathrm{~g} \cdot \mathrm{cm}^{-3}$, obtained from the XRR fit to a 10-nm thick SiN deposited sample, following the method described in Sec. 6.2. Since there are no available references for $\mathrm{N}$, the $\mathrm{N}$ reference peak area is determined following the same method used for $\mathrm{O}$ in Sec. 6.3.1, where the surface Vegard's law must be satisfied for all deposited thicknesses (Eq. 3). A reference value for $\mathrm{N}$ of $330 \pm 23$ counts $\cdot \mathrm{nC}^{-1}$ is obtained, assuming the same reference value for $\mathrm{O}$ of $1170 \pm 70$ counts $\cdot \mathrm{nC}^{-}$ ${ }^{1}$ extracted previously for the $\mathrm{ZrO}_{2}$ on a-Si system. For $\mathrm{ZrO}_{2}$ on $\mathrm{SiO}_{2}$, the used Si surface atomic density is calculated by Eq. 1, by means of a mass density of $2.4 \pm 0.3 \mathrm{~g} \cdot \mathrm{cm}^{-3}$, obtained from the XRR fit to a 10 -nm thick $\mathrm{SiO}_{2}$ deposited sample (see method in Sec. 6.2). Fig. 6.9 shows the evolution of the $\mathrm{Si}$ surface coverage for $\mathrm{ZrO}_{2}$ grown on a-Si (squares), $\mathrm{SiN}$ (triangles) and $\mathrm{SiO}_{2}$ (circles) for high-O (a) and low-O (b) conditions. Note that the $\mathrm{ZrO}_{2}$ thicknesses displayed in Fig. 6.9 are ranging from $0 \mathrm{~nm}$ until the thickness when the $\mathrm{ZrO}_{2}$ layer closes for each system.

Not considering the initial point at $0 \mathrm{~nm}$ deposited $\mathrm{ZrO}_{2}$, a similar reduction of $\mathrm{Si}$ surface coverage with increasing deposited amount of $\mathrm{ZrO}_{2}$ on a-Si, $\mathrm{SiN}$ and $\mathrm{SiO}_{2}$ substrate layers is observed for high-O conditions (Fig. 6.9(a)). Instead, for low-O conditions there is an initial difference in decrease of $\mathrm{Si}$ surface coverage between different substrate layers for $\mathrm{ZrO}_{2}$ thicknesses below $\sim 0.7 \mathrm{~nm}$ (Fig. 6.9(b)). This could be attributed to the strong initial oxidation for high-O conditions which leads to a quick stoichiometric $\mathrm{ZrSiO}_{4}$ formation. Although for low-O conditions, a sub-stoichiometric silicate might be formed at the beginning due to lower amount of oxygen energetic particles, requiring more $\mathrm{ZrO}_{2}$ to achieve the full $\mathrm{ZrSiO}_{4}$ stoichiometry. Note that the different initial decrease of the Si surface coverage observed for low-O conditions on the different substrate layers scales with the Si concentration already present on the surface of either a-Si, $\mathrm{SiN}$ and $\mathrm{SiO}_{2}$, but does not mean that a different sub-stoichiometric silicate is initially formed for each substrate layer. 
For high-O conditions, $\mathrm{ZrO}_{2}$ closes between 3.2 and $3.4 \mathrm{~nm}$, and for low-O conditions, $\mathrm{ZrO}_{2}$ closes between 1.7 and $1.9 \mathrm{~nm}$ on a-Si, $\mathrm{SiN}$ and $\mathrm{SiO}_{2}$, as pointed out by the dashed-dot lines in Figs. 6.9(a) and 6.9(b), respectively. Although there is a large difference (by a factor of $\sim 2$ ) between conditions based on the thickness required to close the $\mathrm{ZrO}_{2}$ layer, there is no apparent effect of $\mathrm{Si}$ passivation (by nitridation or oxidation) on the growth of $\mathrm{ZrO}_{2}$, since the $\mathrm{ZrO}_{2}$ layer closes similarly on top of all three substrate layers for each conditions. One possible explanation to this behavior might be related to thermodynamics. The enthalpies of formation of $\mathrm{ZrO}_{2}$ and specially $\mathrm{ZrSiO}_{4}$ compounds present more negative values when compared to $\mathrm{ZrSi}_{2}, \mathrm{Si}_{3} \mathrm{~N}_{4}$ and $\mathrm{SiO}_{2}$ compounds (see Table 6.1). Since all competing reactions of formation are exothermic, more negative values denote more compound stability. Thus, the $\mathrm{ZrO}_{2}$ growth would not be influenced by having either bare $\mathrm{Si}(\mathrm{a}-\mathrm{Si})$ or passivated $\mathrm{Si}\left(\mathrm{SiN}\right.$ or $\left.\mathrm{SiO}_{2}\right)$ as substrate layers, and $\mathrm{ZrSiO}_{4}$ and/or $\mathrm{ZrO}_{2}$ formation would be the dominant processes.

(a) High-O ZrO

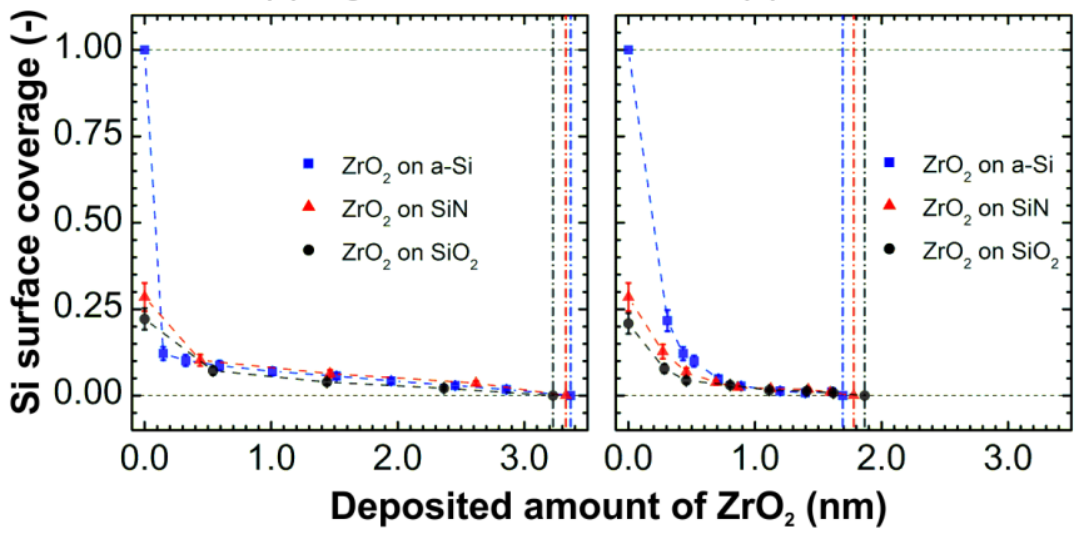

Fig. 6.9. Si surface coverage as a function of deposited amount for $\mathrm{ZrO}_{2}$ grown on a-Si (squares), $\mathrm{SiN}$ (triangles) and $\mathrm{SiO}_{2}$ (circles) for high-O (a) and low-O (b) deposition conditions. The dashed lines through the data points are guides for the eye. The two horizontal dashed in (a) and (b) represent the respective limits for the $\mathrm{Si}$ surface coverage (from 0 to 1). The three vertical dashed-dot lines in (a) and (b) denote the thickness when $\mathrm{ZrO}_{2}$ becomes a closed layer on a-Si (blue), $\mathrm{SiN}$ (red) and $\mathrm{SiO}_{2}$ (black). 
Table 6.1. Standard molar enthalpies of formation $\Delta_{\mathrm{f}} H^{o}$ at $25^{\circ} \mathrm{C}$ in $\mathrm{kJ} \cdot \mathrm{mol}^{-1}$ for $\mathrm{ZrSi}_{2}, \mathrm{Si}_{3} \mathrm{~N}_{4}, \mathrm{SiO}_{2}$, $\mathrm{ZrO}_{2}$ and $\mathrm{ZrSiO}_{4}$ compounds.

\begin{tabular}{ccc}
\hline \hline Compound & $\Delta_{\mathrm{f}} H^{\circ}$ & Ref. \\
\hline $\mathrm{ZrSi}_{2}$ (crys.) & -159.4 & 365 \\
$\mathrm{Si}_{3} \mathrm{~N}_{4}$ (crys.) & -743.5 & 38 \\
$\mathrm{SiO}_{2}$ ( $\alpha$-quarz) & -910.7 & 38 \\
$\mathrm{ZrO}_{2}$ (crys.) & -1100.6 & 38 \\
$\mathrm{ZrSiO}_{4}$ (crys.) & -2033.4 & 38 \\
\hline \hline
\end{tabular}

\subsubsection{Oxygen diffusion response upon thermal oxidation of the $\mathrm{ZrO}_{2} / \mathrm{Si}$ system}

To study oxygen diffusion through $\mathrm{ZrO}_{2}$ upon thermal oxidation, the $\mathrm{ZrO}_{2} / \mathrm{Si}$ system with minor $\mathrm{ZrO}_{2}$ interaction with the underlying a-Si layer already from as deposited is considered. The earlier in vacuo XPS studies (from Sec. 6.3.1) reveal less $\mathrm{Si}$ interaction with the low-O $\mathrm{ZrO}_{2}$ layer. Since only a small amount of Si reacts to form interfacial silicate for such low-O conditions, there is still significant unreacted Si that can be used for monitoring the oxygen diffusion process through the initially formed $\mathrm{ZrSiO}_{4}$ layer. Knowing that $1.7 \mathrm{~nm}$ is needed to close the low-O $\mathrm{ZrO}_{2}$ on a-Si, a thickness of $2 \mathrm{~nm}$ is selected for the $\mathrm{ZrO}_{2}$ layer. Assuming that $\sim 1.7 \mathrm{~nm}$ is mainly related to $\mathrm{ZrSiO}_{4}$, the $\sim 0.3 \mathrm{~nm}$ difference corresponds to $\mathrm{ZrO}_{2}$. Thus, the oxygen diffusion process will not be only through $\mathrm{ZrSiO}_{4}$ but also through pure $\mathrm{ZrO}_{2}$. It should be noted that defective oxide structures can lead to enhanced diffusion, ${ }^{366}$ therefore structural defects of this $\mathrm{ZrO}_{2}$ film should be also evaluated when considering the choice of sample conditions for the diffusion studies. We can indirectly evaluate these defects by determining the stoichiometry and the mass density of this $\mathrm{ZrO}_{2}$ film. Since these parameters for such thin film are difficult to measure experimentally, we consider the parameters from "bulk" $\mathrm{ZrO}_{2}$ films grown by the two deposition conditions. According to the previous in vacuo XPS studies presented in Sec. 6.3.1 for the $10 \mathrm{~nm}$-thick $\mathrm{ZrO}_{2}$ samples, a similar stoichiometry close to bulk $\mathrm{ZrO}_{2}$ is obtained for both conditions, though the high-O conditions present lower density $(\sim 12 \%)$ than the low-O conditions, that exhibit a density value close to bulk $\mathrm{ZrO}_{2}$. This means that the high-O conditions might create a more porous structure with more defects which can lead to higher oxygen diffusion. Hence, apart from the lower reactivity of $\mathrm{ZrO}_{2}$ with a-Si, this is an additional reason to choose the low-O conditions for the diffusion studies.

Oxygen diffusion through the $\mathrm{ZrO}_{2} / \mathrm{ZrSiO}_{4}$ layers is monitored through the increase of the Si-2p XPS peak corresponding to $\mathrm{ZrSiO}_{4}$ and/or $\mathrm{SiO}_{2}$ (from now on named $\mathrm{Si}-2 \mathrm{p}$ 
ox.) and the decrease of the elemental Si-2p peak during thermal oxidation. In addition, the $\mathrm{Zr}-3 \mathrm{~d}$ XPS peaks can also be followed to check the impact on both $\mathrm{ZrO}_{2}$ and $\mathrm{ZrSiO}_{4}$ formed layers. For such study, $2 \mathrm{~nm}$ low-O $\mathrm{ZrO}_{2}$ samples are grown on $5 \mathrm{~nm}$ a-Si layers onto $\mathrm{Si}$ substrates, annealed under atmospheric conditions at different temperatures from as deposited to $600^{\circ} \mathrm{C}$, and measured by ex situ AR-XPS, before and after annealing, to quantify the oxygen diffusion process. Fig. 6.10 shows the evolution of $\mathrm{Zr}-3 \mathrm{~d}$ (a) and Si$2 p$ (b) XPS peaks for several of these samples upon thermal oxidation. Note that the spectra shown in Fig. 6.10 are normalized to the respective main $\mathrm{Zr}-3 \mathrm{~d}$ and elemental Si$2 p$ peaks, after subtraction of a Shirley background. ${ }^{359}$

(a) Zr-3d

(b) Si-2p

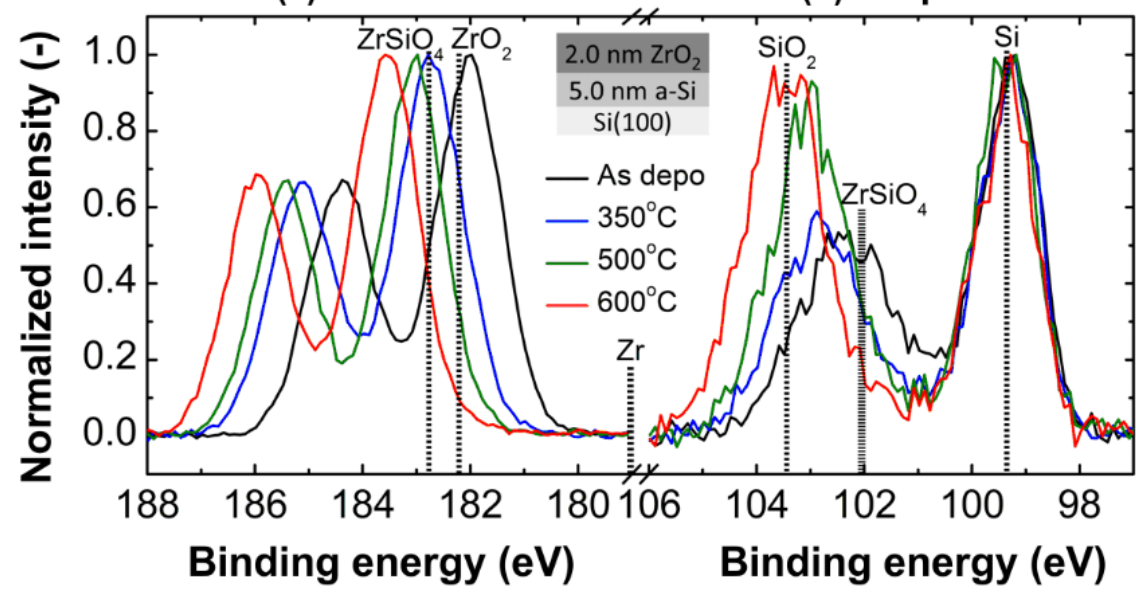

Fig. 6.10. $\mathrm{Zr}-3 \mathrm{~d}$ (a) and $\mathrm{Si}-2 \mathrm{p}$ (b) XPS peaks for low-O $\mathrm{ZrO}_{2}$ on a-Si for several samples annealed during $20 \mathrm{~min}$ at different temperatures from as deposited to $600^{\circ} \mathrm{C}$ (solid lines). $\mathrm{Bulk} \mathrm{Zr}, \mathrm{Si}, \mathrm{ZrO}_{2}$, $\mathrm{SiO}_{2}$ and $\mathrm{ZrSiO}_{4}$ binding energies are marked by dashed lines, and a layered model of the deposited structure is also displayed. Note that the shown spectra are recorded with $34^{\circ}$ off normal detection.

As expected, all zirconium is reacted forming $\mathrm{ZrO}_{2}$ and $\mathrm{ZrSiO}_{4}$ already from as deposited as observed in Fig. 6.10(a) (black spectrum) by the absence of Zr elemental $(\sim 178.9 \mathrm{eV})$ and the formation of a single $\mathrm{Zr}-3 \mathrm{~d}_{5 / 2}$ peak at $\sim 182.0 \mathrm{eV}$ that overlaps with bulk $\mathrm{ZrO}_{2}(\sim 182.3 \mathrm{eV})$. Similar $\mathrm{Si}-2 \mathrm{p}$ peaks are formed for this $2 \mathrm{~nm}$ deposited sample and the previous $1 \mathrm{~nm}$ deposited sample in Sec. 6.3.1, as shown by Fig. 6.10(b) (black spectrum) and Fig. 6.4(b), respectively. Though, double thickness leads to about the double ( $47 \%$ ) of Si reacting with $\mathrm{ZrO}_{2}$ at the interface when compared to the previous sample, which is in line with the LEIS results that the interfacial silicate layer still grows up to $1.7 \mathrm{~nm}$ deposited thickness. However, it seems that the main product of this interfacial reaction is $\mathrm{ZrSiO}_{4}$ due to the position of the Si-2p ox. peak centered at bulk $\mathrm{ZrSiO}_{4}(\sim 102.0 \mathrm{eV})$ (Fig. 6.10(b), black spectrum). Therefore, $\mathrm{SiO}_{2}$ formation might be negligible upon deposition, and only $\mathrm{ZrO}_{2}$ and $\mathrm{ZrSiO}_{4}$ will be formed. 
There is a clear shift towards higher energies of the Si-2p ox. peak without increase in its content for the samples annealed at temperatures higher than as deposited until $400^{\circ} \mathrm{C}$, as represented by the sample annealed at $350^{\circ} \mathrm{C}$ (Fig. 6.10 (b), blue spectrum). At the same time, a shift towards higher energies of the $\mathrm{Zr}-3 \mathrm{~d}$ peaks is observed (see Fig. 6.10(a)). This can be attributed to the enrichment of $\mathrm{Si}$ in the interfacial $\mathrm{ZrSiO}_{4}$ layer during annealing without further silicate formation and/or oxidation of the underlying aSi layer, as confirmed by similar O content from the O-1s peaks (not shown) for the as deposited and annealed samples up to $400^{\circ} \mathrm{C}$. Thus, when the temperature increases, Si atoms become more mobile, diffuse up from the a-Si layer and react with the $\mathrm{ZrSiO}_{4}$ layer, forming a Si-rich silicate. But, within this temperature range, the $\mathrm{ZrO}_{2}$ and silicate layers block diffusion of oxygen to the underlying a-Si layer. Both energy shifts have also been detected in pseudobinary $\left(\mathrm{ZrO}_{2}\right)_{\mathrm{x}}\left(\mathrm{SiO}_{2}\right)_{1-\mathrm{x}}$ alloys with increased $\mathrm{SiO}_{2}$ content, corresponding to a Si-rich silicate. ${ }^{364,367}$ These energy shifts can be associated to the less electron-donating nature (i.e., more electronegative) of the second nearest-neighbor Si relative to $\mathrm{Zr}$, when comparing $\mathrm{Zr}-\mathrm{O}-\mathrm{Si}$ and $\mathrm{Zr}-\mathrm{O}-\mathrm{Zr}$ bond arrangements. This decreased electron donation from the second-nearest neighbor slightly decreases the electron density on the metal atom. The resulting more positive electrostatic potential at the $\mathrm{Zr}$ atom results in an increased binding energy of the $\mathrm{Zr}-3 \mathrm{~d}$ photoelectrons. Similarly, for increasing Si content there will be less electron donation of $\mathrm{Zr}$ atoms to $\mathrm{Si}$, leading to an increase of the binding energy of the Si-2p photoelectrons from the Si-2p ox. peak. ${ }^{367}$

At temperatures above $400^{\circ} \mathrm{C}$, the silicate is no longer blocking the diffusion of oxygen and some oxygen penetrates down and oxidizes the a-Si layer forming additional silicate and eventually also $\mathrm{SiO}_{2}$, as observed by the clear increase and the slight shift to higher energies of the Si-2p ox. peak for the samples annealed at 500 and $600^{\circ} \mathrm{C}$ (see the respective green and red spectra in Fig. 6.10(b)). This energy shift detected for the Si-2p ox. peak is even more pronounced for the $\mathrm{Zr}-3 \mathrm{~d}$ peaks, denoting enhanced Si diffusion at 500 and $600^{\circ} \mathrm{C}$ which results in more Si-rich silicate formation (see green and red spectra in Fig. 6.10(a)). Similar shifts towards higher energies of both Si-2p ox. and Zr$3 \mathrm{~d}$ peaks have been observed by Hwang et al. ${ }^{62}$ for thermal oxidized $\mathrm{ZrO}_{2} / \mathrm{Si}$ films at temperatures between 400 and $700^{\circ} \mathrm{C}$. They also detected a decrease of the elemental Si$2 p$ peak during annealing, which in our case corresponds to the increase of the Si-2p ox. peak relative to the normalized $\mathrm{Si}-2 \mathrm{p}$ peak. In that study, the shift of the $\mathrm{Si}-2 \mathrm{p}$ ox. between 400 and $500^{\circ} \mathrm{C}$ was associated to either $\mathrm{Zr}$ silicate formation ( $\mathrm{Zr}$-O-Si bonds) or thermally grown $\mathrm{Si}$ sub-oxide $\left(\mathrm{SiO}_{\mathrm{x}}, 1<\mathrm{x}<2\right)$. They observed a similar position of the $\mathrm{Si}-2 \mathrm{p}$ ox. peak centered at $\sim 103.5 \mathrm{eV}$ for temperatures between 600 and $700^{\circ} \mathrm{C}$. This was related to stoichiometric $\mathrm{SiO}_{2}$ formation, as also observed in our system at $\sim 600^{\circ} \mathrm{C}$ (Fig. 6.10(b), red spectrum).

To quantitatively estimate the oxygen diffusion through the $2 \mathrm{~nm}$ low-O $\mathrm{ZrO}_{2}$ layer deposited on a-Si upon thermal oxidation, the previous AR-XPS data is analyzed in more 
detail. Note that, as discussed before, the direct oxygen diffusion through $\mathrm{ZrO}_{2}$ cannot be studied, since there is formation of $\mathrm{ZrSiO}_{4}$ just when the deposition starts. Thus, oxygen diffusion through the formed $\mathrm{ZrO}_{2} / \mathrm{ZrSiO}_{4}$ bilayer will be the relevant process, and the thickness evolution of these two layers will be determined for all as deposited and annealed samples.

For quantitative analysis, the $\mathrm{Zr}-3 \mathrm{~d}$ and elemental $\mathrm{Si}-2 \mathrm{p}$ peaks are fitted with a doublet. Since the $\mathrm{Si}-2 \mathrm{p}$ ox. peak is rather broad, it is fitted with a single peak for reasons of simplicity. From these fits, only the $\mathrm{Zr}-3 \mathrm{~d}_{5 / 2}, \mathrm{Si}-2 \mathrm{p}_{3 / 2}$ and $\mathrm{Si}-2 \mathrm{p}$ ox. peaks are considered, and an adapted 2-overlayer model composed by $\mathrm{ZrO}_{2} / \mathrm{ZrSiO}_{4}$ on top of a-Si substrate is used for AR-XPS quantification (see layered model in Fig. 6.11). Simplified, this adapted model is based on the Beer-Lambert equation ${ }^{368}$ and follows a similar method as described by Cumpson and Seah. ${ }^{282}$ Photoelectrons generated at a depth $z$ are attenuated by a factor $e^{-z / \lambda \cos \theta}$, with $\theta$ the detection angle and $\lambda$ the attenuation length. For our specific samples, it has been taken into account that the $\mathrm{Zr}-3 \mathrm{~d}_{5 / 2}$ peak is not only associated to the $\mathrm{ZrO}_{2}$ layer, but also to the $\mathrm{ZrSiO}_{4}$ layer. Thus, in our adapted model the $\mathrm{Zr}-3 \mathrm{~d}_{5 / 2} / \mathrm{Si}-2 \mathrm{p}$. ox. and $\mathrm{Zr}-3 \mathrm{~d}_{5 / 2} / \mathrm{Si}-2 \mathrm{p}_{3 / 2}$ intensity ratios are used to fit the thickness of the $\mathrm{ZrO}_{2}$ layer, while the $\mathrm{ZrSiO}_{4}$ thickness is fitted from the ratio Si-2p ox./Si-2 $\mathrm{p}_{3 / 2}$. The attenuation length $\lambda$ for each XPS peak in each compound (or element) of the layer is calculated according to Cumpson and Seah method ${ }^{282}$ as implemented in the MultiOverlayer Calculator from Thermo Avantage software ${ }^{358}$ using bulk mass density values $\rho$ for each overlayer or substrate compound (or element). The bulk intensity ratio $R_{\mathrm{o}}$ of each overlayer compound relative to the substrate is calculated directly as described in ref. ${ }^{369}$. For that, $\rho, M$ and $\lambda$ for the bulk overlayer and substrate materials are required, where $M$ is the molar mass of the compound (or element) (see all calculated $\lambda$ and $R_{\mathrm{o}}$ values and all used $\rho$ parameters in Table 6.2).

Table 6.2. Adapted 2-overlayer model parameters for AR-XPS quantification.

\begin{tabular}{cccccccc}
\hline \hline $\begin{array}{c}\text { No. } \\
\mathrm{L}^{\mathrm{a}}\end{array}$ & $\mathrm{C}^{\mathrm{b}}$ & $\rho^{\mathrm{c}}$ & $\begin{array}{c}\mathrm{XPS} \\
\text { peak }\end{array}$ & $\begin{array}{c}\lambda^{\mathrm{d}} \text { in } \\
\mathrm{ZrO}_{2}\end{array}$ & $\begin{array}{c}\lambda^{\mathrm{d}} \text { in } \\
\mathrm{ZrSiO}_{4}\end{array}$ & $\begin{array}{c}\lambda^{\mathrm{d}} \text { in } \\
\mathrm{Si}\end{array}$ & $\mathrm{R}_{\mathrm{o}}^{\mathrm{e}}$ \\
\hline 1 & $\mathrm{ZrO}_{2}$ & $5.68^{38}$ & $\mathrm{Zr}-3 \mathrm{~d}_{5 / 2}$ & 1.916 & $\ldots$ & $\ldots$ & 0.361 \\
2 & $\mathrm{ZrSiO}_{4}$ & $4.56^{38}$ & $\mathrm{Zr}-3 d_{5 / 2}$ & 1.916 & 2.045 & $\ldots$ & 0.211 \\
& & & $\mathrm{Si}-2 \mathrm{p}$ ox. & 2.007 & 2.143 & $\ldots$ & 0.221 \\
$\mathrm{Sub}$ & $\mathrm{a}-\mathrm{Si}$ & $2.33^{38}$ & $\mathrm{Si}-2 \mathrm{p}_{3 / 2}$ & 2.007 & 2.144 & 2.904 & $\ldots$ \\
\hline \hline
\end{tabular}

$\mathrm{L}^{\mathrm{a}}$ is the number of the overlayer on the substrate (Sub).

$\mathrm{C}^{\mathrm{b}}$ is the chemical formula of each overlayer or substrate compound (or element). $\rho^{\mathrm{c}}$ is the mass density of each compound (or element) (in $\mathrm{g}^{\cdot} \mathrm{cm}^{-3}$ ).

$\lambda^{\mathrm{d}}$ is the attenuation length of photoelectrons from each XPS peak in each compound (or element) (in $\mathrm{nm}$ ).

$\mathrm{R}_{0}{ }^{\mathrm{e}}$ is the bulk intensity ratio of each overlayer compound relative to the substrate. 
After all input parameters for the model are determined, the two unique free parameters corresponding to the overlayer thicknesses $z$ for the $\mathrm{ZrO}_{2}$ and $\mathrm{ZrSiO}_{4}$ layers can be obtained for each temperature. For that, experimental and simulated peak intensity ratios from the three considered XPS peaks $\left(\mathrm{Zr}-3 \mathrm{~d}_{5 / 2}, \mathrm{Si}-2 \mathrm{p}_{3 / 2}\right.$ and $\mathrm{Si}-2 \mathrm{p}$ ox.) are calculated for each detection angle and annealing temperature. The overlayer thicknesses $z$ for both overlayers are initially estimated and iteratively changed until the simulated and the experimental peak intensity ratios vs. angle match by a least-squares minimization procedure. ${ }^{328}$ A more detailed information of the method for AR-XPS quantification of multi-overlayer-substrate systems can be found in ref. ${ }^{282}$. Note that for this quantification only a range of detection angles between 34 and $49^{\circ}$ off normal is selected, since a wider angular range gives a larger deviation from the model, probably because the interfaces between layers are not ideally sharp, in line with the nearly $2 \mathrm{~nm}$ $\mathrm{ZrO}_{2}$ needed for forming a closed layer. Fig. 6.11 shows the obtained $\mathrm{ZrSiO}_{4}$ (triangles) and the $\mathrm{ZrO}_{2}$ (circles) overlayer thicknesses for low-O $\mathrm{ZrO}_{2}$ annealed samples on a-Si at different temperatures between as deposited and $600^{\circ} \mathrm{C}$.

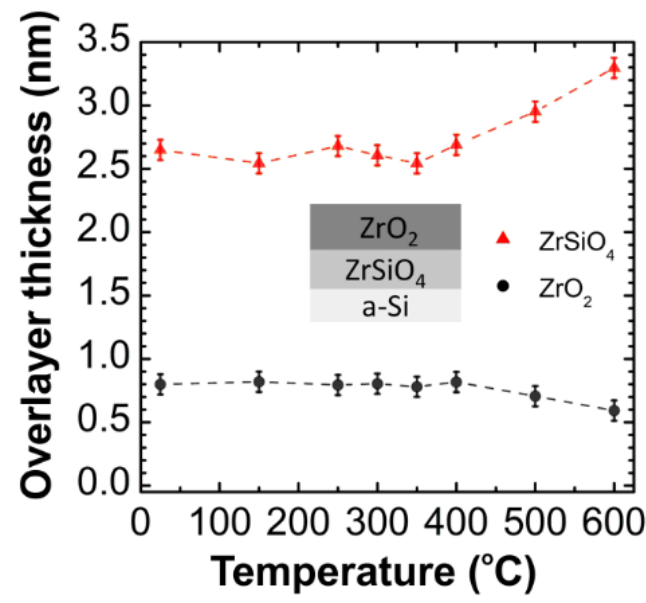

Fig. 6.11. Overlayer thickness for $\mathrm{ZrSiO}_{4}$ (triangles) and $\mathrm{ZrO}_{2}$ (circles) layers on $\mathrm{Si}$ substrate as function of temperature for a $2 \mathrm{~nm}$ low-O $\mathrm{ZrO}_{2}$ sample deposited onto $5 \mathrm{~nm}$ a-Si. The dashed lines through the data points are guides for the eye. The adapted 2-overlayer model on a substrate is also shown.

From as deposited until $\sim 400^{\circ} \mathrm{C}$, both $\mathrm{ZrO}_{2}$ and $\mathrm{ZrSiO}_{4}$ layers present similar overlayer thicknesses, as depicted by circles and triangles in Fig. 6.11, respectively. Average overlayer thicknesses of $0.8 \pm 0.1$ and $2.6 \pm 0.1 \mathrm{~nm}$ are obtained within this temperature range for the respective $\mathrm{ZrO}_{2}$ and $\mathrm{ZrSiO}_{4}$ layers. This means that when $\sim 2$ $\mathrm{nm} \mathrm{ZrO}_{2}$ are deposited on a-Si, slightly more than half of $\mathrm{ZrO}_{2}(\sim 1.2 \mathrm{~nm})$ reacts with $\mathrm{Si}$ (or $\mathrm{SiO}_{\mathrm{x}}$, due to the initial pre-sputtering of $\mathrm{Zr}$ in $\mathrm{Ar}+\mathrm{O}_{2}$ mixture) forming $\mathrm{ZrSiO}_{4}$, and the rest $(\sim 0.8 \mathrm{~nm})$ remains as $\mathrm{ZrO}_{2}$, and no additional oxidation occurs by further $\mathrm{ZrSiO}_{4}$ 
and/or $\mathrm{SiO}_{2}$ formation when the temperature is increased up to $\sim 400^{\circ} \mathrm{C}$. Taking into account the molar volumes of $\mathrm{ZrSiO}_{4}$ and $\mathrm{ZrO}_{2},{ }^{38}$ and the direct reaction between $\mathrm{ZrO}_{2}$ and $\mathrm{SiO}_{2}$ to form $\mathrm{ZrSiO}_{4},{ }^{362}$ we can estimate a volumetric molar ratio of $\mathrm{ZrSiO}_{4} / \mathrm{ZrO}_{2}$ of $\sim 2.0$. If we consider that $\sim 1.2 \mathrm{~nm} \mathrm{ZrO}_{2}$ fully reacts with $\mathrm{SiO}_{2}$, then according to this ratio, about $2.4 \mathrm{~nm} \mathrm{ZrSiO}_{4}$ will be formed, which almost corresponds to the average $\mathrm{ZrSiO}_{4}$ overlayer thickness obtained by our 2-overlayer adapted model $(2.6 \pm 0.1 \mathrm{~nm})$. It should be noted that our 2-overlayer adapted model assumes sharp interfaces between layers, while the LEIS results indicate an error function shaped in-depth concentration profile. ${ }^{353}$ This might lead to certain inaccuracies in the overlayer thickness determination, especially upon annealing.

At temperatures higher than $400^{\circ} \mathrm{C}$, there is a slight increase of the $\mathrm{ZrSiO}_{4}$ overlayer thickness of $\sim 0.3$ and $\sim 0.6 \mathrm{~nm}$ with respect to the as deposited value $(2.6 \pm 0.1 \mathrm{~nm})$, as shown in Fig. 6.11 (triangles) for samples annealed $20 \mathrm{~min}$ at 500 and $600^{\circ} \mathrm{C}$, respectively. At the same time, there is a small decrease of the $\mathrm{ZrO}_{2}$ overlayer thickness of $\sim 0.1$ and $\sim 0.2 \mathrm{~nm}$ compared to the initial value $(0.8 \pm 0.1)$ for 500 and $600^{\circ} \mathrm{C}$ (Fig. 6.11 (circles)). The decrease of the $\mathrm{ZrO}_{2}$ thickness is due to the reaction of $\mathrm{ZrO}_{2}$ with a-Si to form additional $\mathrm{ZrSiO}_{4}$, which results in an increase of the $\mathrm{ZrSiO}_{4}$ layer. This also means that the combined $\mathrm{ZrO}_{2}$ and silicate layer allows oxygen penetration above $400^{\circ} \mathrm{C}$, resulting in formation of more $\mathrm{ZrSiO}_{4}$ and/or $\mathrm{SiO}_{2}$ underneath. It should be noted that the shift in binding energy of the $\mathrm{Zr}$-3d peaks upon annealing (see Fig. 6.10(a)) suggests that all $\mathrm{ZrO}_{2}$ would be incorporated in a Si-rich silicate, while the AR-XPS model indicates that a slightly decreased pure $\mathrm{ZrO}_{2}$ top layer is remaining. This could be related to the fact that the model assumes sharp interfaces between layers, while additional intermixing takes places upon annealing. Furthermore, the formation of a more Si-rich silicate just below a sub-nm $\mathrm{ZrO}_{2}$ surface layer, could also affect the $\mathrm{Zr}-3 \mathrm{~d}$ binding energy through change in electronegativity of the next-nearest neighbor atom, as described previously. ${ }^{364,367}$

There is still a remaining question about the structure of the $\mathrm{ZrO}_{2}$ and a-Si films from the as deposited and annealed low-O $\mathrm{ZrO}_{2}$ samples on a-Si, which is supposed to be amorphous. To verify the amorphous nature of both $\mathrm{ZrO}_{2}$ and a-Si films, XRD measurements (not shown) reveal the presence of an XRD amorphous phase, without appreciable signs of crystallinity, demonstrating the amorphous character of both films in the samples. To confirm this apparent amorphous nature, it is interesting to study the surface topography of these samples. The possible crystallization of the $\mathrm{ZrO}_{2}$ film upon annealing might lead to an increase of the surface roughness due to growth and aggregation of smaller grains, as observed in several thermal oxidation studies of $\mathrm{ZrO}_{2}$ films. ${ }^{355,356}$ Therefore, the surface morphology of our samples is analyzed by AFM. As an example, AFM images of an as deposited sample, and samples annealed at $400^{\circ} \mathrm{C}$ and $500^{\circ} \mathrm{C}$ are presented in Figs. 6.12(a), 6.12(b) and 6.12(c), respectively. 
(a) As deposited

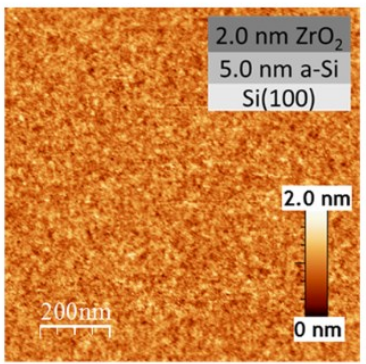

(b) $400^{\circ} \mathrm{C}$

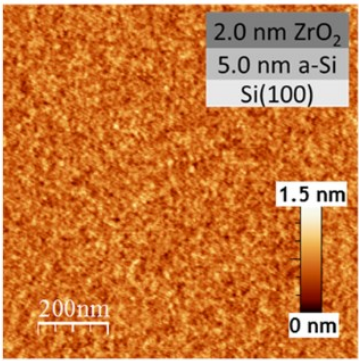

(c) $500^{\circ} \mathrm{C}$

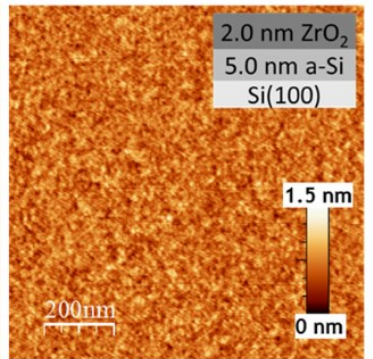

Fig. 6.12. AFM images $(1 \mathrm{x} 1 \mu \mathrm{m})$ of low-O $\mathrm{ZrO}_{2}$ on a-Si an as deposited sample (a) and samples annealed during $20 \mathrm{~min}$ at $400^{\circ} \mathrm{C}$ (b) and $500^{\circ} \mathrm{C}$ (c). Layered models of the deposited structures are shown in insets.

All three as deposited, and annealed samples at $400^{\circ} \mathrm{C}$, and at $500^{\circ} \mathrm{C}$, exhibit similar flat surfaces (see AFM images in Figs. 6.12(a), 6.12(b) and 6.12(c)), with respective RMS roughness values of $0.22 \pm 0.05,0.16 \pm 0.05$, and $0.16 \pm 0.05 \mathrm{~nm}$, close to the uncoated $\mathrm{Si}(100)$ substrate and comparable to the intermediate thickness samples presented in Sec. 6.3.1 (see example in Fig. 6.6(b), for low-O conditions). The small RMS roughness values for all these samples, even reduced for enhanced temperatures due to possible smoothening, confirms the amorphous nature of our $\sim 2.0 \mathrm{~nm}$-thick $\mathrm{ZrO}_{2}$ films. This smoothening effect has also been observed for thin $\mathrm{ZrO}_{2}$ samples annealed at $400^{\circ} \mathrm{C} .{ }^{355}$ However, in that study, samples were annealed under reduced oxygen pressures $\left(\sim 10^{-5}\right.$ mbar), ${ }^{355}$ much lower than in our atmospheric conditions. Still, other annealing studies performed under comparable atmospheric conditions, showed similar surface roughness values $(\sim 0.2 \mathrm{~nm})$ for temperatures up to $600^{\circ} \mathrm{C} .{ }^{356,370}$ In these studies, temperatures $>600^{\circ} \mathrm{C}$ provoked surface morphology changes which led to increased RMS roughness. ${ }^{356,370}$ This was attributed to $\mathrm{ZrO}_{2}$ crystallization occurring at such enhanced temperatures, which could result into an increase of the oxygen diffusion through the $\mathrm{ZrO}_{2}$ film due to the appearance of grain boundaries in its just formed polycrystalline structure. 22,34

It should be noted that crystallization not only may depend on the annealing temperature, but also on the film thickness. A study performed for $\mathrm{ZrO}_{2}$ thin films on $\mathrm{Si}$, revealed that $\sim 7 \mathrm{~nm}$ films started to crystallize at temperatures between 500 and $600^{\circ} \mathrm{C}$, while $\sim 13 \mathrm{~nm}$ films started to crystallize at temperatures below $400^{\circ} \mathrm{C}$. $^{2}$ There, this difference was related to the higher surface/volume ratio of the thinner films that stabilized the amorphous phase for a larger temperature range in order to keep the total Gibbs free energy to the minimum. ${ }^{62}$ In our case, the oxygen diffusion process presented previously, which occurs at temperatures $>400^{\circ} \mathrm{C}$ (see Figs. 6.10 and 6.11), seems not to be motivated by a crystallization process, due to the apparent amorphous nature of our 
films up to $600^{\circ} \mathrm{C}$. Temperatures higher than this value might be required to crystallize such thin $(\sim 2 \mathrm{~nm})$ low-O $\mathrm{ZrO}_{2}$ films on a-Si, where a large $(\sim 2.6 \mathrm{~nm})$ silicate interface is already formed upon deposition (see Fig. 6.11). Thus, further research is needed to elucidate the nature of this oxygen diffusion process at temperatures $>400^{\circ} \mathrm{C}$, which leads to more silicate and/or $\mathrm{SiO}_{2}$ being formed.

\subsection{Conclusions}

The initial growth of DC sputtered $\mathrm{ZrO}_{2}$ thin films on amorphous $\mathrm{Si}$ has been studied for two sputter gas mixtures with high and low oxygen content (High-O and lowO conditions) by in vacuo HS-LEIS. The high surface sensitivity of LEIS makes this technique unique for such studies. Thus, deposited material thicknesses required to close the $\mathrm{ZrO}_{2}$ layer on a-Si have been determined for both deposition conditions. $\mathrm{ZrO}_{2}$ forms a closed layer on a-Si at about 3.4 and $1.7 \mathrm{~nm}$ for high-O and low-O conditions, respectively. The factor 2 reduction of the thickness to close the layer for the low-O conditions was attributed to the reduction of energetic and oxidative particles arriving at the sample, which leads to minor intermixing by silicate formation. Instead for high-O conditions, not only more silicate and/or even $\mathrm{SiO}_{\mathrm{x}}$ was formed, but also $\sim 3 \% \mathrm{Si}$ segregated up towards the surface from the $\mathrm{ZrO}_{2} / \mathrm{a}-\mathrm{Si}$ interface, which contributes to additional intermixing.

Furthermore, in vacuo XPS has been used to determine the stoichiometry and the initial interaction of $\mathrm{ZrO}_{2}$ with a-Si for high-O and low-O conditions. Although both conditions produce layers with similar close to bulk $\mathrm{ZrO}_{2}$ stoichiometry, XRR showed that high-O $\mathrm{ZrO}_{2}$ layers were $\sim 12 \%$ less dense than low-O $\mathrm{ZrO}_{2}$ layers. Thus, high-O conditions lead to a more porous structure with more defects when compared to the other conditions. $\mathrm{ZrSiO}_{4}$ formation was the dominant process occurring at the interface for both conditions. However, for high-O conditions, more silicate and/or $\mathrm{SiO}_{\mathrm{x}}$ was formed due to the higher flux of (energetic) oxygen particles from the magnetron plasma.

The effect of the passivation of the underlying a-Si layer (by nitridation or oxidation) on the initial growth of $\mathrm{ZrO}_{2}$ has also been studied. The results showed absence of such an effect on the $\mathrm{ZrO}_{2}$ growth, since this layer closes similarly on top of a-Si, $\mathrm{SiN}$ and $\mathrm{SiO}_{2}$ substrate layers. This was attributed to the favorable enthalpies of formation of $\mathrm{ZrO}_{2}$ and $\mathrm{ZrSiO}_{4}$ compounds when compared to the enthalpies of formation of the substrate layer compounds.

Finally, oxygen diffusion through $\mathrm{ZrO}_{2}$ films under thermal oxidation has been investigated for a $2 \mathrm{~nm}$ low-O $\mathrm{ZrO}_{2}$ film on a-Si by ex situ AR-XPS. The results did not show increased silicate and/or $\mathrm{SiO}_{2}$ formation at temperatures below $400^{\circ} \mathrm{C}$, despite the large $(\sim 2.6 \mathrm{~nm})$ interfacial silicate formed just upon deposition. At these temperatures, the underlying a-Si was protected against oxidation, with only $\mathrm{Si}$-enrichment of this 
interfacial silicate being detected. In addition, as deposited and thermally oxidized $\mathrm{ZrO}_{2}$ samples exhibited an XRD amorphous phase, and presented a low surface roughness, close to the Si substrate. The nature of the oxygen diffusion process for temperatures $>400^{\circ} \mathrm{C}$ could not be associated to crystallization, and more research is needed to understand this process. However, the oxygen diffusion barrier capabilities, together with the amorphous nature and the smoothness of these samples, make $\mathrm{ZrO}_{2}$ thin films an interesting candidate as oxidation protective capping layers for a-Si and possibly other oxidation sensitive materials.

\section{Acknowledgments}

This work is part of the research programme 'Controlling photon and plasma induced processes at EUV optical surfaces (CP3E)' of the 'Stichting voor Fundamenteel Onderzoek der Materie (FOM)', which is part of and financially supported by the 'Nederlandse Organisatie voor Wetenschappelijk Onderzoek (NWO)'. The CP3E programme is carried out in the Industrial Focus Group XUV Optics, supported by Carl Zeiss SMT, ASML, PANalytical, DEMCON, SolMates, TNO, and the Province of Overijssel. 


\section{Valorization and outlook}

In the last decades, nanometer thin films have been used in numerous applications due to their interesting scale-related properties, mostly different from typical bulk properties, and highly dependent on the material type (i.e., metal, metal-oxide, nitride, etc.). In particular, the first two material type thin films have been applied in several fields due to their interesting associated properties. In the case of metal (e.g., $\mathrm{Pd}, \mathrm{Ru}$, etc.) thin films, these fields include applications such as catalysis, ${ }^{1,2}$ microelectronics (DRAMs and CMOS devices) $^{3,4,5,6}$ and EUVL optics. ${ }^{7,8,9,10}$ Metal-oxide (e.g., $\mathrm{RuO}_{2}$, $\mathrm{ZrO}_{2}$, etc.) thin films are applied in similar applications as the ones mentioned for metallic films, ${ }^{6,9,10,12,14,22,34,40,41}$ as well as photovoltaics, ${ }^{39,45,113}$ or other relevant fields, ${ }^{29,30,31}$ for which their characteristic oxide properties are relevant. In several of these applications such as DRAMs, CMOS devices, solar cells, or optics designed for EUVL, oxygen diffusion towards deeper layers is one of the main threats for their performance. In microelectronics, oxygen diffusion will usually result in a drop of capacitance. $^{22}$ In photovoltaics, this will lead to a common reduction of the UV transmission through reflection and parasitic absorption, and a loss of the photogenerated power via recombination at trap states and electrical resistance losses. ${ }^{45}$ And, in EUVL optics, this will cause a reflectance loss due to EUV absorption. ${ }^{54}$ So, in all these applications the layer underneath (usually $\mathrm{Si}$ ) should not be directly exposed to an oxidative environment.

The research presented in this thesis was motivated by the use of relevant oxidation resistant capping materials for protecting DRAMs, CMOS devices, solar cells and EUV multilayer mirrors against oxidation and other contamination phenomena. This could be useful to enlarge their lifetime, contributing to their current or near future implementation. The research work was conducted within an industrial partnership programme 'Controlling photon and plasma induced processes at EUV optical surfaces (CP3E)', started by FOM (which is now part of NWO), Carl Zeiss SMT and ASML. This program was initiated to meet the need of research on various aspects of EUV optics performance, as a continuation of a previous successful program 'Extreme UV Multilayer Optics (XMO)'. This $\mathrm{PhD}$ project was focused on the understanding of the growth and thermal oxidation (and/or oxygen diffusion) of $\mathrm{Ru}$ and $\mathrm{ZrO}_{2}$ thin films as 
relevant oxidation resistant protective materials for microelectronics, photovoltaics and EUVL optics.

As observed in this thesis (Chapters 5 and 6), the deposition of either $\mathrm{Ru}$ or $\mathrm{ZrO}_{2}$ thin films directly on an a-Si substrate layer, as a typical ending layer of an a-Si solar cell or a Mo/Si multilayer mirror, provoked the formation of a relatively thick intermixed interface, depending on the material and the deposition conditions ( $\sim 5 \mathrm{~nm}$ for $\mathrm{Ru}$ and, $\sim 2-4 \mathrm{~nm}$ for $\mathrm{ZrO}_{2}$, depending on deposition conditions). Knowing that a typical relevant capping layer thickness is usually below $3 \mathrm{~nm},{ }^{40}$ this intermixing region is already significant. It could lead to an important decrease of the transmittance (and photogenerated power) or the reflectance of the respective photovoltaics or EUVL optics due to a respective increased UV absorption (and recombination/electrical losses) or EUV absorption, if larger thicknesses are required. A mitigation strategy to reduce the intermixing, and hence the thickness to form a closed layer, was presented in this thesis. It consisted of passivating the a-Si layer either by nitridation or oxidation. This method worked in the case of $\mathrm{Ru}$, where its closed layer thickness was reduced by increasing $\mathrm{Si}$ passivation, resulting into just $\sim 2 \mathrm{~nm}$ for oxidized $\mathrm{Si}$. In the case of $\mathrm{ZrO}_{2}$, there was no apparent effect of the Si passivation on the thickness to close the layer. This might be improved by means of using another interdiffusion barrier such as $\mathrm{B}_{4} \mathrm{C}$ between both $\mathrm{ZrO}_{2}$ and a-Si layers. The use of a $\mathrm{B}_{4} \mathrm{C}$ barrier has already been proven to work, preventing the intermixing between a $\mathrm{Ru}$ protective layer and an underneath Siterminated multilayer mirror. ${ }^{49}$ We furthermore show that the oxygen content during reactive sputtering (e.g. of $\mathrm{ZrO}_{2}$ ) directly affects the intermixing of the layer with the underlying Si layer. This conclusion is of direct importance for the development of not only $\mathrm{ZrO}_{2}$, but also of other binary oxides, showing that for ultrathin films one cannot engineer layer properties without ignoring their effect on the underlying layer.

It is important to account for the processes affecting the lifetime of capping layers and the underlying structure, for application as solar cells or EUVL mirrors. Exposure to respectively UV sunlight or EUV radiation, may lead to processes such as atmospheric corrosion or EUV-induced oxidation. As explained in more detail in Sec. 1.4.1.1, EUVinduced oxidation presents similarities with thermal oxidation that can be used to understand this phenomenon. Therefore, thermal oxidation of $\mathrm{Ru}$ and $\mathrm{ZrO}_{2}$ thin films was investigated in this thesis (Chapters 3, 4 and 6). The appearance of $\mathrm{RuO}_{2}$ nanocolumns on the $\mathrm{Ru}$ surface at temperatures above $\sim 200^{\circ} \mathrm{C}$ (Chapter 3 ) showed that $\mathrm{Ru}$ thin films could undergo deterioration above these enhanced temperatures. This is relevant when $\mathrm{Ru}$ is used as capping material, since mirrors close to the collector might achieve similar, and even higher temperatures, ${ }^{61}$ and their lifetime might be threatened due to this agglomeration phenomenon. An important information that can help to predict this lifetime, is the detection of the kinetics of diffusion of oxygen through the capping material. The diffusion kinetics and activation energies were determined for oxygen 
diffusion through $\mathrm{RuO}_{2}$ during thermal oxidation of $\mathrm{Ru}$ thin films (Chapter 4). This might be important for understanding how quickly oxygen penetrates the oxide structure, and hence the possible deterioration of the solar cell or the mirror underneath this supposed $\mathrm{RuO}_{2}$ capping material. ${ }^{130,131}$ In the case of thermal oxidation of low-O $\mathrm{ZrO}_{2}$, although silicate formation was already detected upon deposition, the underlying a-Si layer was protected against further silicate and/or oxide formation at temperatures below $400^{\circ} \mathrm{C}$. This might be relevant for its use as oxidation protective material, since the temperatures achieved in the solar cells or the EUVL scanners are lower than this value.

Associated with the last application, the oxidation resistant protective material solutions presented in this thesis can also be applied to protect EUV pellicles against oxidation and other contaminants (i.e. C). For instance, Ru has been proposed as protective layer for EUV pellicles. ${ }^{371}$

As already mentioned previously, there are other applications where both $\mathrm{Ru}$ and $\mathrm{ZrO}_{2}$ thin films can be applied to protect an underneath Si layer against oxidation, where the information learnt in this thesis might be relevant. In the case of $\mathrm{Ru}$, it is used as bottom electrode in capacitors based on high dielectric materials. ${ }^{5,6}$ Its metal-oxide $\left(\mathrm{RuO}_{2}\right)$ can also be applied as bottom gate electrode of oxide dielectric capacitors in DRAMs. ${ }^{6} \mathrm{ZrO}_{2}$ thin films can be used as a replacement of $\mathrm{SiO}_{2}$ gate dielectrics in CMOS, ${ }^{22,33}$ as dielectric layers in DRAMs, ${ }^{34,35}$ or as contact and passivation layers for solar cells. ${ }^{39}$

As stated before, both $\mathrm{Ru}$ and $\mathrm{ZrO}_{2}$ thin films can also be used in diverse applications including protecting layers, diffusion barriers, or, in the case of $\mathrm{Ru}$, catalysts where the information presented in this thesis might also be relevant. For instance, Ru is used as catalyst in ammonia synthesis, ${ }^{1,2}$ or as $\mathrm{Cu}$ diffusion barrier and/or $\mathrm{Cu}$ seed layer in integrated circuits with copper interconnect technology. ${ }^{4}$ Its oxide $\left(\mathrm{RuO}_{2}\right)$ can also be applied as oxidation catalyst in heterogeneous catalysis and electrocatalysis. ${ }^{14}$ In the case of $\mathrm{ZrO}_{2}$, applications as protective layer for UV filters and laser mirrors have been reported. ${ }^{29,30,31}$ 


\section{Summary}

This thesis focuses on the study of the physical and chemical processes occurring during growth and thermal oxidation of $\mathrm{Ru}$ and $\mathrm{ZrO}_{2}$ thin films. Acting as oxidation resistant capping materials, these films are applied in several applications, i.e., in microelectronics such as in gate dielectrics or in memory devices, in photovoltaics, or in extreme ultraviolet lithography (EUVL) optics, where an underneath layer (usually Si) should be protected against oxidation, and are capped by oxidation protective layers. The different properties that these protective layers should possess include film continuity, chemical inertness with the underlying layers, thermal stability, and low-oxidation and/or oxygen diffusion barrier properties. All these properties are addressed in this thesis. In particular, the first two properties are accounted during growth of $\mathrm{Ru}$ and $\mathrm{ZrO}_{2}$ thin films as oxidation protective layers by low-temperature DC magnetron sputtering, and the later three properties are accounted during thermal oxidation of these films. Although thermal oxidation exhibits differences with other oxidation processes such as atmospheric corrosion or EUV-induced oxidation, it is believed that the core physical processes (e.g., oxygen diffusion, sublayer oxidation) are similar, and by understanding both growth and thermal oxidation of such films, the lifetime of the electronics, photovoltaics, and EUVL optics could be improved.

Since most materials, and especially oxides, highly absorb in the EUV range, ultrathin $(<3 \mathrm{~nm})$, usually amorphous, $\mathrm{Ru}$ and $\mathrm{ZrO}_{2}$ films are considered to act as effective oxygen diffusion barriers. To study the growth of such ultrathin films, a high surface sensitive technique which allows an accurate investigation of the initial growth with Ångström precision, is needed. High-sensitivity low-energy ion scattering (HSLEIS) is considered to be unique for these studies due to its sensitivity to the outermost atomic layer. As such, it has been used in this thesis for in vacuo analysis of the initial stages of growth of $\mathrm{Ru}$ and $\mathrm{ZrO}_{2}$ thin films on a-Si, SiN and $\mathrm{SiO}_{2}$ substrate layers, including an accurate determination of surface coverages and thicknesses required for closing the growing film. During $\mathrm{ZrO}_{2}$ growth, the oxygen concentration (high-O and low-O conditions) in the sputter gas was varied in order to study its effect on $\mathrm{ZrO}_{2}$ growth and properties (e.g., stoichiometry, interaction with the underlying layer, mass density). 
The results for $\mathrm{Ru}$ growth studies revealed a large intermixing $(4.7 \mathrm{~nm})$ by silicide formation for $\mathrm{Ru}$ grown on a-Si, which reduced with increasing $\mathrm{Si}$ passivation $(3.2 \mathrm{~nm}$ on $\mathrm{SiN}$, and $2.0 \mathrm{~nm}$ on $\mathrm{SiO}_{2}$ ), mainly attributed to the thermodynamics involved in the compound formation for all three systems. However, the large intermixing for the Ruon-Si system $(4.7 \mathrm{~nm}$ ) could be understood when considering the reverse system (Si-on$\mathrm{Ru}$ ), where only $0.9 \mathrm{~nm}$ intermixing occurred. This difference was predominantly explained by the strong Si surface segregation observed for Ru-on-Si, which was absent for Si-on-Ru. This surface segregation effect was also observed for Ru-on-SiN, but was absent for $\mathrm{Ru}$-on- $\mathrm{SiO}_{2}$. For this last system, an oxygen surface segregation effect was revealed.

In contrast to $\mathrm{Ru}$, for $\mathrm{ZrO}_{2}$ growth on a-Si, $\mathrm{SiN}$ and $\mathrm{SiO}_{2}$, there was a similar intermixing observed on all three substrate layers, attributed to more favorable thermodynamics of formation of $\mathrm{ZrO}_{2}$ and/or $\mathrm{ZrSiO}_{4}$ species, resulting in passivation of the $\mathrm{Si}$ from the substrate layer. The intermixing by silicate formation was reduced by a factor of 2 for low-O conditions $(\sim 1.7 \mathrm{~nm})$ when compared to high-O conditions $(\sim 3.4$ $\mathrm{nm})$. This reduction was related to the decreased amount of energetic and oxidative particles arriving at the substrate for low-O conditions, which led to less silicate being formed.

Besides $\mathrm{Ru}$ and $\mathrm{ZrO}_{2}$ growth studies, these materials have also been annealed in atmospheric oxygen, and their thermal stability, oxidation and/or oxygen diffusion behavior have been investigated. In the case of $\mathrm{Ru}$, a detailed description of surface and sub-surface oxidation of Ru thin films has been presented, and a model for concurrent $2 \mathrm{D}$ and $3 \mathrm{D}$ ruthenium oxide growth has been proposed. In addition, in situ XRR measurements have been used for an accurate determination of the changes of the indepth electron density distribution during thin film $\mathrm{RuO}_{2}$ growth. From this information, diffusion rates and activation energies have been determined for oxygen diffusion through $\mathrm{RuO}_{2}$ thin films.

For thermal oxidation studies of $\mathrm{Ru}$ thin films, 3D $\mathrm{RuO}_{2}$ nano-columns appeared on the surface only above a threshold temperature of $\sim 200^{\circ} \mathrm{C}$, and continued growing in size with annealing temperature and time. However, below this threshold, only $\sim 1$ monolayer of thin film low density $\mathrm{RuO}_{\mathrm{x}}(2<\mathrm{x} \leq 3)$ was formed on the surface. The growth rate of the columns was limited by diffusion of ruthenium from the underlying film. Simultaneously to the growth of the $3 \mathrm{D}$ nano-columns, sub-surface $2 \mathrm{D}$ thin film oxidation continued. In situ XRR allowed to detect that sub-surface oxidation did not proceed by the formation of a single oxide layer, but a combination of two oxide layers on top of each other. Below the mentioned low density $\mathrm{RuO}_{\mathrm{x}}(2<\mathrm{x} \leq 3)$ film, a newly near bulk high density $\mathrm{RuO}_{2}$ film appeared. In the temperature range between 380 and $400^{\circ} \mathrm{C}$, the low density oxide quickly saturated at a thickness of about $3 \mathrm{~nm}$, while the high 
density $\mathrm{RuO}_{2}$ oxide film continued to grow, limited by diffusion of either oxygen or ruthenium through the oxide film. Thus, diffusion rates could be determined for oxygen (and/or ruthenium) through $\mathrm{RuO}_{2}$, and were found not to be constant with the $\mathrm{RuO}_{2}$ thickness. A gradual decrease in diffusion rates was observed with oxide growth. However, for every particular oxide thickness in the mentioned temperature range, the diffusion process seemed to be governed by a single diffusion mechanism with a certain activation energy associated, following a typical linear Arrhenius dependence. From 3.0 to $4.5 \mathrm{~nm}$ films, the activation energy for interdiffusion increased from about 2.1 to 2.4 $\mathrm{eV}$. Since no detectable changes in the density of the $\mathrm{RuO}_{2}$ film were observed, an evolving grain boundary configuration mechanism for this polycrystalline film was suggested as a possible mechanism for slowing interdiffusion.

Finally, thermally oxidized low-O $\mathrm{ZrO}_{2}$ thin films on a-Si showed further intermixing during annealing at the $\mathrm{ZrO}_{2} / \mathrm{Si}$ interface by Si-enrichment of the interfacial silicate initially formed. However, at temperatures below $400^{\circ} \mathrm{C}$, the underlying a-Si was protected against further oxidation. Based on this positive result, $\mathrm{ZrO}_{2}$ might be considered as a promising protective layer for applications where an a-Si film should be protected against oxidation. 


\section{Samenvatting}

Het onderzoek dat in dit proefschrift gepresenteerd wordt, beschrijft de fysische en chemische processen die plaats vinden tijdens groei en thermische oxidatie van $\mathrm{Ru}$ en $\mathrm{ZrO}_{2}$ dunne films. Deze films kunnen worden toegepast als oxidatieresistente toplaag in diverse toepassingen, waaronder micro-elektronica zoals poort diëlektrica of geheugenapparaten, foto-voltaïsche cellen, of extreem ultraviolet lithografie (EUVL) optieken, waar onderliggende lagen (bijv. Si) beschermd moeten worden tegen oxidatie. Belangrijke eigenschappen waar dit soort toplagen aan moeten voldoen zijn continuïteit, chemische inertie naar de onderliggende laag, thermische stabiliteit, en lage affiniteit voor en/of transport van zuurstof. Al deze eigenschappen worden in dit proefschrift behandeld. De eerste twee eigenschappen komen aan bod tijdens de laaggroei van Ru en $\mathrm{ZrO}_{2}$ dunne films, terwijl de laatste eigenschappen aan bod komen tijdens thermische oxidatie-experimenten. Hoewel thermische oxidatie verschilt van andere oxidatie processen zoals atmosferische corrosie of EUV fotongeïnduceerde oxidatie, is het aannemelijk dat een aantal van de fundamentele processen zoals zuurstofdiffusie en onderlaagoxidatie vergelijkbaar zijn. Het begrijpen van groei en thermische oxidatie kan daardoor leiden tot verbetering van de levensduur tijdens toepassing van deze lagen.

Doordat de meeste materialen, en in het bijzonder oxides, een hoge absorptiecoëfficiënt hebben in het EUV-gebied, worden uiterst dunne $(<3 \mathrm{~nm})$, vaak amorfe, $\mathrm{Ru}$ en $\mathrm{ZrO}_{2}$ lagen beschouwd als potentieel effectieve oxidatiebarrières. Om de groei van dit soort zeer dunne lagen in detail te bestuderen is een analysetechniek nodig die beschikt over zeer hoge oppervlaktegevoeligheid. Hoge gevoeligheid lage energie ionenverstrooiing (HS-LEIS) wordt door de specifieke gevoeligheid voor de buitenste atoomlaag beschouwd als een uniek geschikte techniek om deze systemen te bestuderen. HS-LEIS is daarom in dit proefschrift gebruikt om in vacuo de groei van $\mathrm{Ru}$ en $\mathrm{ZrO}_{2}$ lagen te bestuderen, met aandacht voor de oppervlaktebedekking tijdens de groei, en de minimale dikte die nodig is om de laag te sluiten. Tijdens $\mathrm{ZrO}_{2}$ groei is de zuurstofconcentratie in het sputtergas gevarieerd om het effect op de groei en de laageigenschappen (bijv. stoichiometrie, interactie met onderlaag, dichtheid) te bestuderen. 
Voor $\mathrm{Ru}$ groei is ontdekt dat er een grote interactie $(4.7 \mathrm{~nm})$ is tussen $\mathrm{Ru}$ en de onderliggende Si laag, waarbij deze interactie reduceerde wanneer het onderliggende $\mathrm{Si}$ gepassiveerd werd (resp. $3.2 \mathrm{~nm}$ en $2.0 \mathrm{~nm}$ voor $\mathrm{SiN}$ en $\mathrm{SiO}_{2}$ onderlagen), wat gerelateerd werd aan de formatie-energieën van de betrokken verbindingen. De zeer hoge interactie van $\mathrm{Ru}$ met het onderliggend $\mathrm{Si}(4.7 \mathrm{~nm})$ staat in groot contrast met de interactie van Si met onderliggend $\mathrm{Ru}$, waar deze interactie slechts $0.9 \mathrm{~nm}$ blijkt te zijn. Het verschil wordt verklaard door de segregatie van Si naar het oppervlak tijdens $\mathrm{Ru}$ groei op Si, iets wat afwezig is tijdens Si groei op Ru. Deze segregatie naar het oppervlak is ook waargenomen tijdens $\mathrm{Ru}$ groei op $\mathrm{SiN}$, maar is afwezig voor $\mathrm{Ru}$ groei op $\mathrm{SiO}_{2}$. Voor dit laatste systeem werd wel segregatie van zuurstof naar het oppervlak gezien.

In sterk contrast met $\mathrm{Ru}$, werd voor $\mathrm{ZrO}_{2}$ groei geen verschil gezien in interactie met de onderliggende $\mathrm{Si}$ laag voor verschillende oppervlaktepassivaties $\left(\mathrm{Si}, \mathrm{SiN}, \mathrm{SiO}_{2}\right)$, wat toegewezen wordt aan de formatie van energetisch voordelige $\mathrm{ZrO}_{2}$ en/of $\mathrm{ZrSiO}_{4}$ interlagen voor alle oppervlaktepassivaties. De formatie van deze $\sim 3.4 \mathrm{~nm}$ dikke interlagen werd gereduceerd tot $\sim 1.7 \mathrm{~nm}$ wanneer een veel lagere zuurstof partieeldruk werd gebruikt tijdens het reactief sputteren van $\mathrm{Zr}$, iets wat toegekend wordt aan de verminderde hoeveelheid hoogenergetische en oxidatieve deeltjes onder lage partieeldrukken van zuurstof.

De gegroeide lagen zijn verder onderworpen aan atmosferische oxidatie door middel van thermische verhitting. Voor $\mathrm{Ru}$ is een gedetailleerd model ontworpen dat de oxidatie aan en onder het oppervlak beschrijft met simultane 2D en 3D groei van $\mathrm{Ru}$ oxide. Uit harde X-ray metingen tijdens de verhitting is informatie over diffusiesnelheden en activatie-energieën voor transport van zuurstof door $\mathrm{RuO}_{2}$ verkregen.

$3 \mathrm{D} \mathrm{RuO}_{2}$ nanostructuren verschenen aan het oppervlak van $\mathrm{Ru}$ boven een kritische temperatuur van $\sim 200^{\circ} \mathrm{C}$, en deze structuren ontwikkelden zich tijdens verdere thermische verhitting, waarbij de groei gelimiteerd werd door de aanvoer van Ru uit de onderliggende film. Naast de groei van 3D oppervlaktestructuren, trad ook een 2D groei van een $\mathrm{RuO}_{\mathrm{x}}(2<\mathrm{x}<3)$ film op die zich verder voortzette in de diepte. Uit X-ray reflectiemetingen bleek dat deze lage dichtheid $\mathrm{RuO}_{\mathrm{x}}$ film net onder het oppervlak bleef groeien, terwijl zich daar weer onder een hogere dichtheid $\mathrm{RuO}_{2}$ film vormde. De groei van de lage dichtheid oxidelaag stopte na het bereiken van een dikte van $3 \mathrm{~nm}$ (in de experimenten tussen 380 en $400^{\circ} \mathrm{C}$ ), terwijl de hoge dichtheid oxidefilm daaronder continu bleef groeien. De gemeten diffusiesnelheden lieten zien dat de diffusiecontante afnam tijdens oxidegroei. Wanneer de diffusieconstanten geanalyseerd werden als functie van de temperatuur bij een gelijkwaardige oxidedikte, bleek dat de oxide laaggroei aan Arrhenius voldeed, waarbij de activatie-energie toenam van $2.1 \mathrm{eV}$ naar $2.4 \mathrm{eV}$ tijdens groei van de oxidelaag van 3 tot $4.5 \mathrm{~nm}$. Aangezien er geen veranderingen 
in de dichtheid waargenomen zijn, wordt een ontwikkelende kristalkorrelstructuur gesuggereerd als reden voor de veranderende activatie-energie.

Ten slotte is tijdens verhitting van $\mathrm{ZrO}_{2}$ films gezien dat het $\mathrm{ZrO}_{2} / \mathrm{a}-\mathrm{Si}$ grensvlak verder vermengt door $\mathrm{Si}$ verrijking van de silicaat grenslaag. Onder $400^{\circ} \mathrm{C}$ wordt de a$\mathrm{Si}$ laag onder dit grensvlak echter niet verder aangetast. Hierdoor is $\mathrm{ZrO}_{2}$ onder $400^{\circ} \mathrm{C}$ een mogelijk geschikte kandidaat in gebruik als beschermende toplaag tegen Si oxidatie. 


\section{Resumen}

La investigación desarrollada en esta tesis se basa en el estudio de los procesos físicos y químicos que ocurren durante el crecimiento y la oxidación térmica de láminas delgadas de $\mathrm{Ru}$ y $\mathrm{ZrO}_{2}$. Estas láminas se utilizan como recubrimientos resistentes a la oxidación en diversas aplicaciones, e.g. en dispositivos microelectrónicos tales como en dieléctricos de puerta o en dispositivos de memoria, en células fotovoltaicas, o en ópticos de litografía ultravioleta extrema (EUVL), dónde la capa de debajo (normalmente Si) debe estar protegida contra la oxidación, y por ello, se protegen con capas resistentes a la oxidación. Estas capas de protección deben poseer varias propiedades, entre las cuales, se incluyen: la continuidad de la lámina, la baja interacción química con las capas de debajo, la estabilidad térmica, y la baja oxidación y/o las propiedades como barrera a la difusión de oxígeno. Todas estas propiedades se tienen en cuenta en esta tesis. En particular, las dos primeras propiedades se consideran durante el crecimiento de láminas delgadas de $\mathrm{Ru}$ y $\mathrm{ZrO}_{2}$, como capas de protección, por el método de evaporación a bajas temperaturas por pulverización catódica por magnetrón DC. Las tres últimas propiedades se consideran durante la oxidación térmica de estas láminas. Aunque la oxidación térmica presenta diferencias frente a otros procesos de oxidación tales como la corrosión atmosférica o la oxidación inducida por luz ultravioleta extrema, los principales procesos físicos que ocurren (e.g. difusión de oxígeno, oxidación de la subcapa), se consideran similares, y entendiendo ambos procesos de crecimiento y de oxidación térmica de estas láminas, se puede prolongar el tiempo de vida de los dispositivos electrónicos, fotovoltaicos, y ópticos de EUVL.

Teniendo en cuenta que la mayoría de los materiales, y especialmente óxidos, presentan una elevada absorción en el extremo ultravioleta, láminas ultra-delgadas $(<3$ $\mathrm{nm}$ ), normalmente amorfas, de $\mathrm{Ru}$ y $\mathrm{ZrO}_{2}$ se consideran como efectivas barreras a la difusión de oxígeno. Para estudiar el crecimiento de dichas láminas, se requiere una técnica de alta sensibilidad superficial, que permita una investigación del crecimiento inicial con una elevada precisión (a nivel de Ångström). La espectrometría de alta sensibilidad de dispersión de iones de baja energía (HS-LEIS) se considera única para estos estudios, debido a su alta sensibilidad a la última capa atómica. De esta manera, se ha utilizado en esta tesis para el análisis in vacuo de las etapas iniciales de crecimiento 
de láminas delgadas de $\mathrm{Ru}$ y $\mathrm{ZrO}_{2}$ en amorfos sustratos de $\mathrm{Si}, \mathrm{SiN}$ y $\mathrm{SiO}_{2}$, incluyendo una precisa determinación de la composición atómica de la superficie y de los espesores requeridos para cerrar la lámina en crecimiento. Durante el crecimiento de $\mathrm{ZrO}_{2}$, la concentración de oxígeno (condiciones de alto-O y bajo-O) en el gas utilizado para la pulverización por magnetrón se ha variado para estudiar su efecto en el crecimiento y las propiedades (e.g. la estequiometría, la interacción química con la capa de debajo, la densidad) del $\mathrm{ZrO}_{2}$.

Los resultados para los estudios de crecimiento de $\mathrm{Ru}$ mostraron un gran entremezclado $\left(4.7 \mathrm{~nm}\right.$ ), debido a la formación de $\mathrm{RuSi}_{\mathrm{x}}$ para el crecimiento de $\mathrm{Ru}$ en amorfo Si, el cual se redujo incrementando la pasivación del Si (3.2 nm en SiN, y $2.0 \mathrm{~nm}$ en $\mathrm{SiO}_{2}$ ), mayoritariamente debido a la termodinámica involucrada en la formación de los compuestos para los tres sistemas. No obstante, el gran entremezclado para el sistema de Ru-en-Si (4.7 nm) pudo ser comprendido cuando se consideró el sistema reverso (Sien-Ru), donde sólo un entremezclado de $0.9 \mathrm{~nm}$ ocurrió. Esta diferencia pudo ser principalmente explicada por la fuerte segregación de Si hacia la superficie, observada para Ru-en-Si, pero ausente para Si-en-Ru. Este efecto de segregación también se observó para Ru-en-SiN, pero fue ausente para $\mathrm{Ru}$-en- $\mathrm{SiO}_{2}$. Para este último sistema, se reveló un efecto de segregación de oxígeno hacia la superficie.

Al contrario de $\mathrm{Ru}$, para el crecimiento de $\mathrm{ZrO}_{2}$ en a-Si, SiN y $\mathrm{SiO}_{2}$, se apreció un entremezclado similar en los tres sustratos, debido a la favorable termodinámica de formación de $\mathrm{ZrO}_{2}$ y/o $\mathrm{ZrSiO}_{4}$, que resultó en la pasivación de Si del sustrato. El entremezclado causado por la formación de silicato se redujo a la mitad para las condiciones de bajo-O $(\sim 1.7 \mathrm{~nm})$ en comparación con las condiciones de alto-O $(\sim 3.4$ $\mathrm{nm})$. Esta reducción se relacionó al descenso de la cantidad de partículas energéticas y oxidantes llegando al sustrato para las condiciones de bajo-O, provocando una disminución del silicato formado.

Aparte de los estudios de crecimiento de $\mathrm{Ru}$ y $\mathrm{ZrO}_{2}$, estos materiales también se han recocido en oxígeno atmosférico, y se ha investigado su estabilidad térmica, oxidación y/o difusión de oxígeno. En el caso de $\mathrm{Ru}$, se ha presentado una descripción detallada de la oxidación de la superficie y en profundidad de láminas delgadas de $\mathrm{Ru}, \mathrm{y}$ se ha propuesto un modelo para el crecimiento simultáneo en 2D y en 3D de óxido de rutenio. Asimismo, medidas in situ de reflexión de rayos X (XRR) se han utilizado para una determinación precisa de los cambios en profundidad de la densidad electrónica durante el crecimiento de láminas delgadas de $\mathrm{RuO}_{2}$. De esta información, se han determinado las constantes de difusión y las energías de activación para la difusión de oxígeno a través de láminas delgadas de $\mathrm{RuO}_{2}$.

Para los estudios de oxidación térmica de láminas delgadas de $\mathrm{Ru}, 3 \mathrm{D}$ nanocolumnas de $\mathrm{RuO}_{2}$ aparecieron en la superficie sólo en temperaturas por encima de un 
límite de temperatura de $\sim 200^{\circ} \mathrm{C}$, y continuaron creciendo en tamaño cuando la temperatura y el tiempo de recocido se incrementaron. Sin embargo, por debajo de este límite de temperatura, sólo $\sim 1$ monocapa de $\mathrm{RuO}_{\mathrm{x}}(2<\mathrm{x} \leq 3)$ de baja densidad se formó en la superficie. El crecimiento de las columnas se halló limitado por la difusión de rutenio proveniente de la lámina de debajo. Simultáneamente al crecimiento de las 3D nanocolumnas, oxidación en 2D de las láminas delgadas de Ru continuó en profundidad. Medidas in situ de reflexión de rayos $\mathrm{X}$ permitieron detectar que la oxidación en profundidad no procedió con la formación de una simple capa de óxido, pero con la formación de dos capas de óxido, una encima de la otra. Por debajo de la mencionada capa de $\mathrm{RuO}_{\mathrm{x}}(2<\mathrm{x} \leq 3)$ de baja densidad, apareció una nueva capa de $\mathrm{RuO}_{2}$ con densidad cerca de la aparente. En el rango de temperaturas entre 380 y $400^{\circ} \mathrm{C}$, la capa de óxido de baja densidad se saturó rápidamente con un espesor de $\sim 3 \mathrm{~nm}$, mientras que la capa de $\mathrm{RuO}_{2}$ con densidad cerca de la aparente continuó creciendo, con su crecimiento limitado por la difusión de oxígeno o rutenio a través de la capa de óxido. De esta manera, las constantes de difusión se determinaron para la difusión de oxígeno (y/o rutenio) a través de $\mathrm{RuO}_{2}$, y se detectaron variables y dependientes del espesor. Se observó una disminución gradual de las constantes de difusión con el crecimiento del óxido. Sin embargo, para cada particular espesor del óxido en el rango de temperaturas mencionado, el proceso de difusión pareció estar gobernado por un simple mecanismo de difusión con una cierta energía de activación asociada, siguiendo una típica dependencia lineal descrita por Arrhenius. Para espesores del óxido entre 3.0 y $4.5 \mathrm{~nm}$, la energía de activación para el proceso de interdifusión incrementó des de 2.1 a $2.4 \mathrm{eV}$. Teniendo en cuenta que no se detectaron cambios en la densidad de la capa de $\mathrm{RuO}_{2}$, se sugirió un mecanismo consistente en la evolución de la configuración de los límites de grano como posible mecanismo de ralentización del proceso de interdifusión.

Finalmente, las láminas delgadas de $\mathrm{ZrO}_{2}$ en a-Si oxidadas térmicamente en condiciones de bajo-O, mostraron un mayor entremezclado en la interfaz entre $\mathrm{ZrO}_{2}$ y Si durante el recocido, con un enriquecimiento en $\mathrm{Si}$ del silicato formado inicialmente en la interfaz. Sin embargo, para temperaturas por debajo de $400^{\circ} \mathrm{C}$, la lámina inferior de a-Si se halló protegida frente a mayor oxidación. A raíz de dicho resultado positivo, se podría valorar el uso de la lámina delgada de $\mathrm{ZrO}_{2}$ como recubrimiento de protección prometedor para aplicaciones dónde una lámina de a-Si debe protegerse contra la oxidación. 


\section{Acknowledgments}

During my Chemical Engineering Degree in Barcelona, I never thought about pursuing a PhD. However, this idea changed when I did my Final Research Project within the IRS Group at Delft University of Technology. During this project, the way of doing research and the scientific approach of my supervisors, both Herman Kramer and Somnath Kadam, inspired me to change my mind in the direction of research. Therefore, as soon as I returned to Barcelona, I began applying online for $\mathrm{PhD}$ positions in the Netherlands, while I was doing several research projects in my former university. Eventually, I found an interesting and challenging experimental topic to develop my $\mathrm{PhD}$ in the Netherlands. Not only the $\mathrm{PhD}$ topic itself was challenging, but also the fact that it was in a new field to me (Applied Physics), and industrially oriented. I am very thankful to my promotor prof. Fred Bijkerk, who supported and encouraged me, materializing this idea, by becoming a PhD student in September 2012 within his group, formerly named nSI at the FOM Institute DIFFER (in Nieuwegein). He helped me within the project, and also with the movement in June 2013 to the University of Twente, becoming part of the just later founded Industrial Focus Group XUV Optics, within the MESA+ Institute for Nanotechnology in mid-2014.

During my four years of $\mathrm{PhD}$ project, I was lucky to have Robbert van de Kruijs, as my daily supervisor and co-promotor. He motivated and helped me with a good guidance, by teaching me a new technology and the physics behind that. His motivation for good science also became my motivation, and his critical attitude about scientific topics, helped me to consolidate my scientific skills. Apart from his regular supervision, I had the support from Andrey Yakshin and Marko Sturm with scientific discussions in several topics during my $\mathrm{PhD}$. I would like to give a special thanks for their time spent in my project. I still remember the support by Andrey in the elaboration of my first letter, and the trial for the second one, which at the end became a regular journal paper. And also, the nice time spent together in the E-MRS conference in Lille. I remember the interesting discussions about XPS and LEIS that I had with Marko, his involvement during the last two papers related to both techniques, and his persistent help, teaching me about several scientific topics. And also, I cannot forget the nice time that we had in 
Barcelona, together with Julia, showing him my home city and surroundings. Even in the final stage, he helped me with the design of the thesis cover, for which I am very grateful.

I would also like to express my gratitude to all my group colleagues, for their support, knowledge and kindness during my $\mathrm{PhD}$. (Personal elaborate acknowledgments will follow). Here, I would like to give a special thanks to Dima and Andrey, with whom I shared the room at the University of Twente almost during three years. I will not forget the nice time spent together, including personal and scientific discussions. Ah!, and our trip to Mallorca! I want to thank Sasha, for his always positive and motivating attitude. I also would like to thank Qiushi which began his Postdoc at the same time that I started my PhD. I cannot forget living with him in Nieuwegein, a perfect and recommendable experience. I also cannot forget Rolf, present during this period. His cheerfulness, positive and helping attitude were really motivating. And, I would like to thank both group secretaries, Jacqueline and Carin, for their support and help during my $\mathrm{PhD}$ period.

My PhD could not have been accomplished without the contributions of Gisela von Blanckenhagen and Harmut Enkisch (from Carl Zeiss SMT), Philipp Brüner and Thomas Grehl (from ION-TOF GmbH), prof. Hidde Brongersma (from Calipso BV), and Rico Keim (from MESA+ Nanolab). They helped in my research in different aspects: by orienting me in the development of EUVL capping materials, by training me in the features of the Qtac ${ }^{100}$ HS-LEIS setup, by teaching me the LEIS technique, and by helping in the HR-TEM data analysis, respectively. In addition, I would like to thank Saskia and Erwin for their respective assistance in XRD and XPS data analysis. Thank you!

I would like to give a special thanks to my family and friends that supported me through all my PhD. Thanks to Germaine for our regular talks and good advices. Thanks also to both Lydia and Harry for their professional and career advices. Thanks to Aku for the nice time sharing the apartment in Enschede, and to Jose and Gerry, for having a second family in the Netherlands. Also, to my new friends Rio and Ester, and to my new family: Dana, Gerrit, and Emil. Thanks also to all my friends supporting from Spain, specially to Javi, always staying at my side, and also Núria and Emanuel for their good friendship. I don't have words to express my profound gratitude to my parents, my grandma, and my uncle for their help during all the $\mathrm{PhD}$ journey. I enjoyed the extensive time that my mum spent around, with nice food and company. All people from the department will never forget her cakes! Finally, I would like to thank my lovely Julia for the incalculable support, help and affection that she gave me during this last year. Her encouragement was unimaginable. Gracias a todos! 


\section{Curriculum Vitae}

Roger Coloma Ribera was born on $31^{\text {st }}$ October 1986 in Sant Joan Despí, Barcelona, Spain. In 2004, he completed his A-Levels (High School) with honors in the modality of Applied Sciences at Llor School, Sant Boi de Llobregat, Barcelona, Spain. From 2004 to 2011, he pursued a Chemical Engineering Degree at IQS School of Engineering, Ramon Llull University, Barcelona, Spain. While pursuing this degree, he got the opportunity to carry out several internships at different companies, including Alcon Cusí (Novartis), Iberpotash (ICL-Group), and Cromogenia-Units. In 2011, he obtained this degree with an average mark of 8 . The degree project was on designing a propylene glycol production plant. Just after the degree graduation, he went to the Netherlands during six months to develop his Final Research Project at the Intensified Reaction \& Separation Systems Group of the Delft University of Technology, under the supervision of dr. ir. Herman Kramer and dr. Somnath Kadam. The topic of the project was on crystal nucleation by metastable zone width (MSZW) measurements at different volumes and cooling rates. He presented this project in Barcelona at his former university IQS School of Engineering, Ramon Llull University, where he obtained the IQS title, with honors. In October 2011, he got involved in several research projects and teaching assistant tasks within the Chemical Engineering Department of his former university IQS School of Engineering, Ramon Llull University. In September 2012, he moved to the Netherlands, and started his PhD research in Applied Physics at the Nanolayer Surfaces and Interfaces (nSI) Group of the FOM Institute DIFFER, Nieuwegein, under the supervision of Prof. dr. Fred Bijkerk. In June 2013, he was relocated together with the rest of the nSI Group from DIFFER to the University of Twente, being part of the later founded Industrial Focus Group XUV Optics, within the MESA+ Institute for Nanotechnology in mid-2014. The results of this PhD research, carried out as a part of the Industrial Partnership Programme CP3E, are presented in this thesis. 


\section{Bibliography}

${ }^{1}$ O. Hinrichsen, F. Rosowski, M. Muhler, and G. Ertl, Chemical Engineering Science 51 (10), 1683 (1996).

${ }^{2}$ K. Honkala, A. Hellman, I. N. Remediakis, A. Logadottir, A. Carlsson, S. Dahl, C. H. Christensen, and J. K. Norskov, Science 307 (5709), 555 (2005).

${ }^{3}$ M. Subhramannia, B. K. Balan, B. R. Sathe, I. S. Mulla, and V. K. Pillai, The Journal of Physical Chemistry C 111 (44), 16593 (2007).

${ }^{4}$ S. H. Hsieh, W. Chen, and C. M. Chien, Nanomaterials 5 (4), 1840 (2015).

${ }^{5}$ A. Grill, W. Kane, J. Viggiano, M. Brady, and R. Laibowitz, Journal of Materials Research 7 (12), 3260 (1992).

${ }^{6}$ Q.A. Acton, Chemical Processes-Advances in Research and Application: 2013 Edition: ScholarlyBrief. (ScholarlyEditions, 2013).

${ }^{7}$ E. Louis, A. E. Yakshin, T. Tsarfati, and F. Bijkerk, Progress in Surface Science 86 (11-12), 255 (2011).

${ }^{8}$ T. Tsarfati, E. Zoethout, R.W.E. van de Kruijs, and F. Bijkerk, Surface Science 603 (7), 1041 (2009).

${ }^{9}$ H. Lee, J. Choi, S. Koh, J. Kim, D. Kim, J. Choi, H. Kim, H. Ko, B. G. Kim, and C. Jeon, ECS Transactions 58 (6), 93 (2013).

${ }^{10} \mathrm{~S}$. Wurm, in 30th European Mask and Lithography Conference (Proc. SPIE, Dresden, Germany, 2014), Vol. 9231, pp. 923103.

${ }^{11}$ H. J. T. Ellingham, Journal of the Society of Chemical Industry 63 (5), 125 (1944).

${ }^{12}$ J. Jae, W. Zheng, A. M. Karim, W. Guo, R. F. Lobo, and D.G. Vlachos, ChemCatChem 6 (3), 848 (2014).

${ }^{13}$ E. Torun, C. M. Fang, G. A. de Wijs, and R. A. de Groot, The Journal of Physical Chemistry C 117 (12), 6353 (2013).

${ }^{14}$ H. Over, Chemical Reviews 112 (6), 3356 (2012).

${ }^{15}$ Y. Murakami, J. Li, D. Hirose, S. Kohara, and T. Shimoda, Journal of Materials Chemistry C (2015).

${ }^{16}$ J. H. Kim, J. H. Ahn, S. W. Kang, J. S. Roh, S. H. Kwon, and J. Y. Kim, Current Applied Physics 12, S160 (2012).

${ }^{17}$ A. Krause, Ultrathin Calcium Titanate Capacitors: Physics and Application. (Logos Verlag Berlin, 2014).

${ }^{18}$ Y. Shih, K. Lee, and Y. S. Huang, Applied Surface Science 294, 29 (2014).

${ }^{19}$ M. Copel, M. Gribelyuk, and E. Gusev, Applied Physics Letters 76 (4), 436 (2000).

${ }^{20}$ M. C. Zeman, C. C. Fulton, G. Lucovsky, R. J. Nemanich, and W.-C. Yang, Journal of Applied Physics 99 (2), 023519 (2006).

${ }^{21}$ H. Döscher, G. Lilienkamp, P. Iskra, M. Kazempoor, and W. Daum, Journal of Vacuum Science \& Technology B 28 (4), C5B5 (2010). 
${ }^{22}$ G. D. Wilk, R. M. Wallace, and J. M. Anthony, Journal of Applied Physics 89 (10), 5243 (2001).

${ }^{23}$ P. Kondaiah, G. Mohan Rao, and S. Uthanna, Journal of Physics: Conference Series 390 (1), 012031 (2012).

${ }^{24}$ A. Hojabri, Journal of Theoretical and Applied Physics, 1 (2016).

${ }^{25}$ M. A. Mamun, H. Baumgart, and A. A. Elmustafa, ECS Journal of Solid State Science and Technology 4 (5), Q35 (2015).

${ }^{26}$ G. Reyna-García, M. García-Hipólito, J. Guzmán-Mendoza, M. Aguilar-Frutis, and C. Falcony, Journal of Materials Science: Materials in Electronics 15 (7), 439 (2004).

${ }^{27}$ R. Yusoh, M. Horprathum, P. Eiamchai, P. Chindaudom, and K. Aiempanakit, Procedia Engineering 32, 745 (2012).

${ }^{28}$ Y. Chen, A. Li, Y. Li, J. Li, G. Dai, O. Fangping, and X. Xiong, RSC Advances 5 (45), 35929 (2015).

${ }^{29}$ D. Garoli, M. Alaibac, and M. G. Pelizzo, Optica Applicata 44 (4), 611 (2014).

${ }^{30}$ M. Reese, B. Schäfer, P. Großmann, A. Bayer, K. Mann, T. Liese, and H. U. Krebs, Applied Physics A 102 (1), 85 (2011).

${ }^{31}$ T. Liese, V. Radisch, I. Knorr, M. Reese, P. Grossmann, K. Mann, and H. U. Krebs, Applied Surface Science 257 (12), 5138 (2011).

${ }^{32}$ K. J. Hubbard and D. G. Schlom, Journal of Materials Research 11 (11), 2757 (1996).

${ }^{33}$ W. Zhang, Y. Cui, Z. G. Hu, W. L. Yu, J. Sun, N. Xu, Z. F. Ying, and J. D. Wu, Thin Solid Films 520 (20), 6361 (2012).

${ }^{34}$ D. Panda and T. Y. Tseng, Thin Solid Films 531, 1 (2013).

${ }^{35}$ J. P. Chang and Y. S. Lin, Journal of Applied Physics 90 (6), 2964 (2001).

${ }^{36} \mathrm{~V}$. Bakshi, EUV Lithography. (Society of Photo Optical, 2009).

${ }^{37}$ M. Pachecka, J. M. Sturm, R. W. E. van de Kruijs, C. J. Lee, and F. Bijkerk, AIP Advances 6 (7), 075222 (2016).

${ }^{38}$ D.R. Lide, CRC Handbook of Chemistry and Physics: A Ready-reference Book of Chemical and Physical Data. (CRC Press, 2004).

${ }^{39}$ B. Demaurex, J. P. Seif, S. Smit, B. Macco, W. M. M. Kessels, J. Geissbühler, S. De Wolf, and C. Ballif, IEEE Journal of Photovoltaics 4 (6), 1387 (2014).

${ }^{40}$ S. Bajt, N. V. Edwards, and T. E. Madey, Surface Science Reports 63 (2), 73 (2008).

${ }^{41}$ M. Kriese, Y. Platonov, J. Rodriguez, G. Fournier, S. Grantham, C. Tarrio, J. Curry, S. Hill, and T. Lucatorto, in Extreme Ultraviolet (EUV) Lithography VI (Proc. SPIE, San Jose, California, United States 2015), Vol. 9422, pp. 94220K.

${ }^{42}$ D. Ugur, A. J. Storm, R. Verberk, J. C. Brouwer, and W. G. Sloof, Applied Surface Science 288, 673 (2014).

${ }^{43}$ A. I. Kingon, J. P. Maria, and S. K. Streiffer, Nature 406 (6799), 1032 (2000).

${ }^{44}$ H. J. Queisser and E. E. Haller, Science 281 (5379), 945 (1998).

${ }^{45}$ S. Calnan, Coatings 4 (1), 162 (2014).

${ }^{46}$ Y. Liu, P. Stradins, H. Deng, J. Luo, and S. H. Wei, Applied Physics Letters 108 (2), 022101 (2016).

${ }^{47}$ M. Z. Rahman and S. I. Khan, Materials for Renewable and Sustainable Energy 1 (1), 1 (2012).

${ }^{48}$ A. G. Aberle, Progress in Photovoltaics: Research and Applications 8 (5), 473 (2000).

${ }^{49}$ S. Bajt, H. N. Chapman, N. Nguyen, J. Alameda, J. C. Robinson, M. Malinowski, E. Gullikson, A. Aquila, C. Tarrio, and S. Grantham, Appl. Opt. 42 (28), 5750 (2003).

${ }^{50}$ M. Morita, T. Ohmi, E. Hasegawa, M. Kawakami, and K. Suma, Applied Physics Letters 55 (6), 562 (1989). 
${ }^{51}$ J. H. Underwood, E. M. Gullikson, and K. Nguyen, Applied optics 32 (34), 6985 (1993).

${ }^{52}$ R. Kurt, Patent No. US7405031 B2 (29 Jul 2008).

${ }^{53}$ J. Chen, E. Louis, C. J Lee, H. Wormeester, R. Kunze, H. Schmidt, D. Schneider, R. Moors, W. van Schaik, M. Lubomska, and F. Bijkerk, Optics express 17 (19), 16969 (2009).

${ }^{54}$ T. E. Madey, N. S. Faradzhev, B. V. Yakshinskiy, and N. V. Edwards, Applied Surface Science 253 (4), 1691 (2006).

${ }^{55}$ D. C. Shin, M. R. Kim, J. H. Lee, B. H. Choi, and H. K. Lee, Journal of nanoscience and nanotechnology 12 (7), 5631 (2012).

${ }^{56}$ G. Jegert, A. Kersch, W. Weinreich, and P. Lugli, Journal of Applied Physics 109 (1), 014504 (2011).

${ }^{57}$ M. Pešić, S. Knebel, M. Geyer, S. Schmelzer, U. Böttger, N. Kolomiiets, V. V. Afanas'ev, K. Cho, C. Jung, J. Chang, H. Lim, T. Mikolajick, and U. Schroeder, Journal of Applied Physics 119 (6), 064101 (2016).

${ }^{58}$ S. Gieraltowska, B. S. Witkowski, M. Godlewski, and E. Guziewicz, Optica Applicata 43 (1), 17 (2013).

${ }^{59}$ S. Yulin, T. Feigl, T. Kuhlmann, N. Kaiser, A. I. Fedorenko, V. V. Kondratenko, O. V. Poltseva, V. A. Sevryukova, A. Yu. Zolotaryov, and E. N. Zubarev, Journal of Applied Physics 92 (3), 1216 (2002).

${ }^{60}$ S. Tamulevičius, Vacuum 51 (2), 127 (1998).

${ }^{61}$ J. P. Allain, M. Nieto, M. Hendricks, S. S. Harilal, and A. Hassanein, in International Congress on Optics and Optoelectronics (Prague, Czech Republic, 2007), pp. 65860W.

${ }^{62}$ S. M. Hwang, S. M. Lee, K. Park, M. S. Lee, J. Joo, J. H. Lim, H. Kim, J. J. Yoon, and Y. D. Kim, Applied Physics Letters 98 (2), 022903 (2011).

${ }^{63}$ X. Zhao and D. Vanderbilt, Physical Review B 65 (7), 075105 (2002).

${ }^{64}$ J. Robertson, The European physical journal applied physics 28 (3), 265 (2004).

${ }^{65}$ L. Ding, M. Benkhaira, S. Nicolay, and C. Ballif, MRS Proceedings 1426, 51 (2012).

${ }^{66} \mathrm{~J}$. Chen, E. Louis, R. Harmsen, T. Tsarfati, H. Wormeester, M. van Kampen, W. van Schaik, R. W. E. van de Kruijs, and F. Bijkerk, Applied Surface Science 258 (1), 7 (2011).

${ }^{67}$ H. Kim, P. Choi, K. Kim, H. Kuh, D. Beak, J. Lee, J. Yi, and B. Choi, Journal of nanoscience and nanotechnology 14 (5), 3561 (2014).

${ }^{68}$ P. Raghu, C. Yim, F. Shadman, and E. Shero, AIChE Journal 50 (8), 1881 (2004).

${ }^{69}$ M. Miyashita, S. Kawai, and A. Masuda, Japanese Journal of Applied Physics 51 (10S), 10NF12 (2012).

${ }^{70} \mathrm{P}$. Stratton, International Heat Treatment and Surface Engineering 7 (2), 70 (2013).

${ }^{71}$ W. Greiner, D. Rischke, L. Neise, and H. Stöcker, Thermodynamics and Statistical Mechanics. (Springer New York, 2012).

${ }^{72} \mathrm{P}$. Atkins and J. de Paula, Atkins' Physical Chemistry. (OUP Oxford, 2010).

${ }^{73} \mathrm{P}$. Keil, D. Lützenkirchen-Hecht, and R. Frahm, AIP Conference Proceedings 882 (1), 490 (2007).

${ }^{74}$ A. S. Khanna, Introduction to High Temperature Oxidation and Corrosion. (ASM International, 2002).

${ }^{75}$ H. Y. H. Chan, S. Zou, and M. J. Weaver, The Journal of Physical Chemistry B 103 (50), 11141 (1999).

${ }^{76}$ H. Luo, S. Park, H. Y. H. Chan, and M. J. Weaver, The Journal of Physical Chemistry B 104 (34), 8250 (2000).

${ }^{77}$ P. Patnaik, Handbook of inorganic chemicals. (McGraw-Hill New York, 2003). 
${ }^{78}$ R. L. Richards, in Encyclopedia of Inorganic Chemistry (John Wiley \& Sons, Ltd, 2006).

${ }^{79}$ R. J. D. Tilley and B. G. Hyde, Journal of Physics and Chemistry of Solids 31 (7), $1613(1970)$.

${ }^{80}$ D. S. Su and R. Schlögl, Catalysis Letters 83 (3), 115 (2002).

${ }^{81}$ E. Fromm, Kinetics of Metal-Gas Interactions at Low Temperatures: Hydriding, Oxidation, Poisoning. (Springer Berlin Heidelberg, 2012).

${ }^{82}$ DoITPoMS, (University of Cambridge, U.K., 2004-2015); http://www.doitpoms.ac.uk/tlplib/ellingham diagrams/interactive.php).

${ }^{83}$ J. P. Coughlin, Heats and Free Energies of Formation of Inorganic Oxides. (U.S. Government Printing Office, 1954).

${ }^{84}$ B. Chen, R. Jha, H. Lazar, N. Biswas, J. Lee, B. Lee, L. Wielunski, E. Garfunkel, and V. Misra, IEEE Electron Device Letters 27 (4), 228 (2006).

${ }^{85}$ S. H. Oh, C. G. Park, and C. P. Park, Thin Solid Films 359 (1), 118 (2000).

${ }^{86}$ S. Yamamichi, P. Lesaicherre, H. Yamaguchi, K. Takemura, S. Sone, H. Yabuta, K. Sato, T. Tamura, K. Nakajima, S. Ohnishi, K. Tokashiki, Y. Hayashi, Y. Kato, Y. Miyasaka, M. Yoshida, and H. Ono, IEEE Transactions on Electron Devices 44 (7), 1076 (1997).

${ }^{87}$ R. Schmiedl, V. Demuth, P. Lahnor, H. Godehardt, Y. Bodschwinna, C. Harder, L. Hammer, H. P. Strunk, M. Schulz, and K. Heinz, Applied Physics A 62 (3), 223 (1996).

${ }^{88}$ D. Lim, R. Haight, M. Copel, and E. Cartier, Applied Physics Letters 87 (7), 072902 (2005).

${ }^{89}$ R. P. Pezzi, M. Copel, M. Gordon, E. Cartier, and I. J. R. Baumvol, Applied Physics Letters 88 (24), 243509 (2006).

${ }^{90}$ J. Y. Kim, M. C. Nielsen, E. J. Rymaszewski, and T. M. Lu, Journal of Applied Physics 87, 1448 (2000).

${ }^{91}$ R. Nakamura, T. Toda, S. Tsukui, M. Tane, M. Ishimaru, T. Suzuki, and H. Nakajima, Journal of Applied Physics 116 (3), 033504 (2014).

${ }^{92}$ M. Gurvitch, L. Manchanda, and J. M. Gibson, Applied Physics Letters 51 (12), 919 (1987).

${ }^{93}$ J. Kwo, M. Hong, B. Busch, D. A. Muller, Y. J. Chabal, A. R. Kortan, J. P. Mannaerts, B. Yang, P. Ye, H. Gossmann, A. M. Sergent, K. K. Ng, J. Bude, W. H. Schulte, E. Garfunkel, and T. Gustafsson, Journal of Crystal Growth 251 (1-4), 645 (2003).

${ }^{94}$ C. C. Cheng, C. H. Chien, G. L. Luo, J. C. Liu, C. C. Kei, D. R. Liu, C. N. Hsiao, C. H. Yang, and C. Y. Chang, Journal of The Electrochemical Society 155 (10), G203 (2008).

${ }^{95}$ E. P. Gusev, M. Copel, E. Cartier, I. J. R. Baumvol, C. Krug, and M. A. Gribelyuk, Applied Physics Letters 76 (2), 176 (2000).

${ }^{96}$ M. Copel, E. Cartier, E. P. Gusev, S. Guha, N. Bojarczuk, and M. Poppeller, Applied Physics Letters 78 (18), 2670 (2001).

${ }^{97}$ L. Kang, B. H. Lee, W. J. Qi, Y. Jeon, R. Nieh, S. Gopalan, K. Onishi, and J. C. Lee, IEEE Electron Device Letters 21 (4), 181 (2000).

${ }^{98}$ L. V. Goncharova, M. Dalponte, T. Feng, T. Gustafsson, E. Garfunkel, P. S. Lysaght, and G. Bersuker, Physical Review B 83 (11), 115329 (2011).

${ }^{99}$ S. Ferrari and G. Scarel, Journal of Applied Physics 96 (1), 144 (2004).

${ }^{100}$ S. Ferrari and M. Fanciulli, The Journal of Physical Chemistry B 110 (30), 14905 (2006). 
${ }^{101}$ S. K. Kim and C. S. Hwang, Electrochemical and Solid-State Letters 11 (3), G9 (2008).

${ }^{102}$ B. W. Busch, W. H. Schulte, E. Garfunkel, T. Gustafsson, W. Qi, R. Nieh, and J. Lee, Physical review B 62 (20), R13290 (2000).

${ }^{103}$ G. Bakradze, L. P. H. Jeurgens, T. Acartürk, U. Starke, and E. J. Mittemeijer, Acta Materialia 59 (20), 7498 (2011).

${ }^{104}$ S. K. Kim, W. D. Kim, K. M. Kim, C. S. Hwang, and J. Jeong, Applied Physics Letters 85 (18), 4112 (2004).

${ }^{105}$ K. F. Albertin, M. A. Valle, and I. Pereyra, Journal Integrated Circuits and Systems 2 (2), 89 (2007).

${ }^{106}$ A. L. Campbell, H. S. Kim, D. C. Gilmer, B. He, T. Ma, and W. L. Gladfelter, IBM Journal of Research and Development 43 (3), 383 (1999).

${ }^{107}$ O. S. Kwon, S. K. Kim, M. Cho, C. S. Hwang, and J. Jeong, Journal of The Electrochemical Society 152 (4), C229 (2005).

${ }^{108}$ C. S. Hwang, Materials Science and Engineering: B 56 (2), 178 (1998).

${ }^{109}$ M. L. Green, E. P. Gusev, R. Degraeve, and E. L. Garfunkel, Journal of Applied Physics 90 (5), 2057 (2001).

${ }^{110}$ T. Lauinger, J. Schmidt, A. G. Aberle, and R. Hezel, Applied Physics Letters 68 (9), 1232 (1996).

${ }^{111}$ J. Schmidt, F. Werner, B. Veith, D. Zielke, S. Steingrube, P. P. Altermatt, S. Gatz, T. Dullweber, and R. Brendel, Energy Procedia 15, 30 (2012).

${ }^{112}$ B. Hoex, J. J. H. Gielis, M. C. M. van de Sanden, and W. M. M. Kessels, Journal of Applied Physics 104 (11), 113703 (2008).

${ }^{113}$ J. A. Van Delft, D. Garcia-Alonso, and W. M. M. Kessels, Semiconductor Science and Technology 27 (7), 074002 (2012).

${ }^{114}$ T. W. Hamann, O. K. Farha, and J. T. Hupp, The Journal of Physical Chemistry C 112 (49), 19756 (2008).

${ }^{115}$ T. C. Tien, F. N. Pan, L. P. Wang, C. H. Lee, Y. L. Tung, S. Y. Tsai, C. Lin, F. Y. Tsai, and S. J. Chen, Nanotechnology 20 (30), 305201 (2009).

${ }^{116}$ M. Shanmugam, M. F. Baroughi, and D. Galipeau, Thin Solid Films 518 (10), 2678 (2010).

${ }^{117}$ L. J. Antila, M. J. Heikkilä, V. Aumanen, M. Kemell, P. Myllyperkiö, M. Leskelä, and J. E. I. Korppi-Tommola, The Journal of Physical Chemistry Letters 1 (2), 536 (2010).

${ }^{118}$ N. Tétreault, É. Arsenault, L. P. Heiniger, N. Soheilnia, J. Brillet, T. Moehl, S. Zakeeruddin, G. A. Ozin, and M. Grätzel, Nano Letters 11 (11), 4579 (2011).

${ }^{119}$ T. C. Li, M. S. Góes, F. Fabregat-Santiago, J. Bisquert, P. R. Bueno, C. Prasittichai, J. T. Hupp, and T. J. Marks, The Journal of Physical Chemistry C 113 (42), 18385 (2009).

${ }^{120}$ L. M. Do, M. Oyamada, A. Koike, E. M. Han, N. Yamamoto, and M. Fujihira, Thin Solid Films 273 (1), 209 (1996).

${ }^{121}$ M. S. Weaver, L. A. Michalski, K. Rajan, M. A. Rothman, J. A. Silvernail, J. J. Brown, P. E. Burrows, G. L. Graff, M. E. Gross, P. M. Martin, M. Hall, E. Mast, C. Bonham, W. Bennett, and M. Zumhoff, Applied Physics Letters 81 (16), 2929 (2002).

${ }^{122}$ D. S. Wuu, T. N. Chen, E. Lay, C. H. Liu, C. H. Chang, H. F. Wei, L. Y. Jiang, H. U. Lee, and Y. Y. Chang, Journal of The Electrochemical Society 157 (2), C47 (2010).

${ }^{123}$ W. Cao, J. Li, H. Chen, and J. Xue, PHOTOE 4 (1), 040990 (2014).

${ }^{124}$ H. Jung, H. Jeon, H. Choi, G. Ham, S. Shin, and H. Jeon, Journal of Applied Physics 115 (7), 073502 (2014). 
${ }^{125}$ Y. Duan, F. Sun, Y. Yang, P. Chen, D. Yang, Y. Duan, and X. Wang, ACS Applied Materials \& Interfaces 6 (6), 3799 (2014).

${ }^{126}$ D. Yu, Y. Q. Yang, Z. Chen, Y. Tao, and Y. F. Liu, Optics Communications 362, 43 (2016).

${ }^{127}$ G. Balakrishnan, T. N. Sairam, V. R. Reddy, P. Kuppusami, and J. I. Song, Materials Chemistry and Physics 140 (1), 60 (2013).

${ }^{128}$ S. Bajt, J. B. Alameda, J. T. W. Barbee, W. M. Clift, J. A. Folta, B. Kaufmann, and E. A. Spiller, Optical Engineering 41 (8), 1797 (2002).

${ }^{129}$ M. G. Pelizzo, M. Suman, G. Monaco, P. Nicolosi, and D. L Windt, Optics express 16 (19), 15228 (2008).

${ }^{130}$ S. Matsunari, Y. Kakutani, T. Aoki, S. Kawata, and K. Murakami, in Alternative Lithographic Technologies, edited by F. M. Schellenberg and B. M. La Fontaine (Proc. SPIE, San Jose, California, USA, 2009), Vol. 7271, pp. 72713R.

${ }^{131}$ S. Yulin, N. Benoit, T. Feigl, N. Kaiser, M. Fang, and M. Chandhok, in Emerging Lithographic Technologies XII, edited by F. M. Schellenberg (Proc. SPIE, San Jose, California, USA, 2008), Vol. 6921, pp. 692118.

${ }^{132}$ S. Bajt, Z. R. Dai, E. J. Nelson, M. A. Wall, J. B. Alameda, N. Q. Nguyen, S. L. Baker, J. C. Robinson, J. S. Taylor, A. Aquila, and N. V. Edwards, Journal of Micro/Nanolithography, MEMS, and MOEMS 5 (2), 023004 (2006).

${ }^{133}$ T. Tsarfati, E. Zoethout, R. W. E. van de Kruijs, and F. Bijkerk, Surface Science 603 (16), 2594 (2009).

${ }^{134}$ L. Belau, J. Y. Park, T. Liang, H. Seo, and G. A. Somorjai, Journal of Vacuum Science \& Technology B 27 (4), 1919 (2009).

${ }^{135}$ K. Seshan, Handbook of Thin Film Deposition. (Elsevier Science, 2012).

${ }^{136}$ P.K.S.K.S.S. Harsha, Principles of Vapor Deposition of Thin Films. (Elsevier Science, 2005).

${ }^{137}$ W.D. Westwood, Sputter deposition. (AVS, 2003).

${ }^{138}$ S. M. George, Chemical reviews 110 (1), 111 (2009).

${ }^{139}$ J. R. Creighton and P. Ho, Chemical vapor deposition 2, 1 (2001).

${ }^{140}$ J. Shin, D. Gay, Y.-M. Sun, J. M. White, and J. G. Ekerdt, AIP Conference Proceedings 788 (1), 482 (2005).

${ }^{141}$ A. S. Alagoz, J.D. Kamminga, S. Y. Grachev, T.M. Lu, and T. Karabacak, in 2009 MRS Fall Meeting \& Exhibit, edited by E. Lilleodden J. Lou, B. Boyce, L. Lu, P.M. Derlet, D. Weygand, J. Li, M.D. Uchic, E. Le Bourhis (MRS Online Proceedings Library, Boston, Massachusetts, USA, 2009), Vol. 1224, pp. 27.

${ }^{142}$ Q. Wang, J. G. Ekerdt, D. Gay, Y.-M. Sun, and J. M. White, Applied Physics Letters 84 (8), 1380 (2004).

${ }^{143}$ V. Y. Vasilyev, Russian Microelectronics 39 (4), 262 (2010).

${ }^{144}$ S. V. Trubin, N. B. Morozova, P. P. Semyannikov, A. Bessonov, N. V. Gelfond, and I. K. Igumenov, ECS Transactions 25 (8), 881 (2009).

${ }^{145}$ L. Pasquali, N. Mahne, M. Montecchi, V. Mattarello, and S. Nannarone, Journal of Applied Physics 105 (4), 044304 (2009).

${ }^{146}$ A. E. Yakshin, R. W. E. Van De Kruijs, I. Nedelcu, E. Zoethout, E. Louis, F. Bijkerk, H. Enkisch, and S. Müllender, in Advanced Lithography (International Society for Optics and Photonics, 2007), pp. 65170I.

${ }^{147}$ E. Louis, H. J. Voorma, N. B. Koster, L. Shmaenok, F. Bijkerk, R. Schlatmann, J. Verhoeven, Y. Y. Platonov, G. E. van Dorssen, and H. A. Padmore, Microelectronic Engineering 23 (1), 215 (1994). 
${ }^{148}$ M. Ye, Z. Liu, P. Ding, S. Hung, and K. Ahmed, Journal of Vacuum Science \& Technology B: Microelectronics and Nanometer Structures Processing, Measurement, and Phenomena 24 (5), 2214 (2006).

${ }^{149}$ H. N. Alshareef, H. C. Wen, H. F. Luan, K. Choi, H. R. Harris, Y. Senzaki, P. Majhi, B. H. Lee, B. Foran, and G. Lian, Thin Solid Films 515 (4), 1294 (2006).

${ }^{150}$ S. Mahieu, P. Ghekiere, D. Depla, and R. De Gryse, Thin Solid Films 515 (4), 1229 (2006).

${ }^{151}$ A. Rodriguez-Navarro, W. Otañno-Rivera, J. M. Garcia-Ruiz, R. Messier, and L. J. Pilione, Journal of materials research 12 (07), 1689 (1997).

${ }^{152}$ D. Depla, S. Mahieu, and J. Greene, Handbook of deposition technologies for films and coatings: science, applications and technology, 253 (2010).

${ }^{153}$ W.M. Stacey, Fusion Plasma Physics. (Wiley, 2012).

${ }^{154}$ G. Koster, M. Huijben, and G. Rijnders, Epitaxial Growth of Complex Metal Oxides. (Elsevier Science, 2015).

${ }^{155}$ D. Coada, M. Nagl, Th. Lechleitner, and H. Pulker, Romanian Reports in Physics 56 (3), 328 (2004).

${ }^{156}$ R. Alvarez, J. M. Garcia-Martin, M. C. Lopez-Santos, V. Rico, F. J Ferrer, J. Cotrino, A. R. Gonzalez-Elipe, and A. Palmero, Plasma Processes and Polymers 11 (6), 571 (2014).

${ }^{157}$ S. Venkataraj, O. Kappertz, H. Weis, R. Drese, R. Jayavel, and M. Wuttig, Journal of Applied Physics 92 (7), 3599 (2002).

${ }^{158}$ T. Prathyusha, C. S. Reddy, P. S. Reddy, and A. S. Reddy, International Journal of ChemTech Research 6 (6), 3349 (2014).

${ }^{159} \mathrm{~V}$. Nirupama and S. Uthanna, Journal of Materials Science: Materials in Electronics 21 (1), 45 (2009).

${ }^{160}$ P. Pansila, N. Witit-anun, and S. Chaiyakun, Procedia Engineering 32, 862 (2012).

${ }^{161}$ W. Li, X. Yan, A. G. Aberle, and S. Venkataraj, Japanese Journal of Applied Physics 54 (8S1), 08KC14 (2015).

${ }^{162}$ H. Matsui, H. Toyoda, and H. Sugai, Journal of Vacuum Science \& Technology A 23 (4), 671 (2005).

${ }^{163}$ Z. Zhang and M. G. Lagally, Science 276 (5311), 377 (1997).

${ }^{164}$ J. A. Venables, G. D. T. Spiller, and M. Hanbucken, Reports on Progress in Physics 47 (4), 399 (1984).

${ }^{165}$ G. H. Meier, Thermodynamics of Surfaces and Interfaces: Concepts in Inorganic Materials. (Cambridge University Press, Cambridge, 2014).

${ }^{166}$ James F. Ziegler, M. D. Ziegler, and J. P. Biersack, Nuclear Instruments and Methods in Physics Research Section B: Beam Interactions with Materials and Atoms 268 (1112), 1818 (2010).

${ }^{167}$ R. S. Averback and T. D. De La Rubia, in Solid State Physics, edited by Ehrenreich Henry and Spaepen Frans (Academic Press, 1998), Vol. Volume 51, pp. 281.

${ }^{168}$ C. Zhang, X. Kuang, Y. Jin, C. Lu, D. Zhou, P. Li, G. Bao, and A. Hermann, ACS Applied Materials \& Interfaces 7 (48), 26776 (2015).

${ }^{169}$ R. M. Tromp and J. B. Hannon, Surface Review and Letters 09 (03n04), 1565 (2002).

${ }^{170}$ D. L. Beke, G. A. Langer, G. Molnár, G. Erdélyi, G. L. Katona, A. Lakatos, and K. Vad, Philosophical Magazine 93 (16), 1960 (2013).

${ }^{171}$ A. Portavoce and G. Tréglia, Physical Review B 85 (22), 224101 (2012).

${ }^{172}$ D. Gupta, in Diffusion Processes in Advanced Technological Materials (Springer Berlin Heidelberg, Berlin, Heidelberg, 2005), pp. 1. 
${ }^{173}$ A. M. Gusak, T. V. Zaporozhets, Y. O. Lyashenko, S. V. Kornienko, M. O. Pasichnyy, and A. S. Shirinyan, Diffusion-controlled Solid State Reactions: in Alloys, Thin-Films, and Nanosystems. (John Wiley \& Sons, 2010).

${ }^{174}$ J. Musil, P. Baroch, J. Vlček, K. H. Nam, and J. G. Han, Thin solid films 475 (1), 208 (2005).

${ }^{175}$ P. A. Dowben and A. Miller, Surface Segregation Phenomena. (Taylor \& Francis, 1990).

${ }^{176}$ J. Nyéki, C. Girardeaux, Z. Erdélyi, G. A. Langer, G. Erdélyi, D. L. Beke, and A. Rolland, Surface science 495 (3), 195 (2001).

${ }^{177}$ S. Swaminarayan and D. J. Srolovitz, Acta materialia 44 (5), 2067 (1996).

${ }^{178}$ I. Jäger, Surface science 376 (1), 219 (1997).

${ }^{179}$ J. P. Muscat, Journal of Physics C: Solid State Physics 15 (4), 867 (1982).

${ }^{180}$ S. Tirumala, Integration of Ferroelectric Materials into High Density Non-Volatile Random Access Memories. (Virginia Polytechnic Institute and State University, 2000).

${ }^{181}$ S. K. Kim, J. H. Han, G. H. Kim, and C. S. Hwang, Chemistry of Materials 22 (9), 2850 (2010).

${ }^{182}$ Q. Xie, Y. L. Jiang, J. Musschoot, D. Deduytsche, C. Detavernier, R. L. Van Meirhaeghe, S. Van den Berghe, G. P. Ru, B. Z. Li, and X. P. Qu, Thin Solid Films 517 (16), 4689 (2009).

${ }^{183}$ K. Kukli, M. Kemell, E. Puukilainen, J. Aarik, A. Aidla, T. Sajavaara, M. Laitinen, M. Tallarida, J. Sundqvist, M. Ritala, and M. Leskelä, Journal of The Electrochemical Society 158 (3), D158 (2011).

${ }^{184}$ M. Geidel, M. Junige, M. Albert, and J. W. Bartha, Microelectronic Engineering 107, 151 (2013).

${ }^{185}$ J. H. Han, S. W. Lee, G. J. Choi, S. Y. Lee, C. S. Hwang, C. Dussarrat, and J. Gatineau, ECS Transactions 19 (2), 717 (2009).

${ }^{186}$ R. L. Puurunen, W. Vandervorst, W. F. A. Besling, O. Richard, H. Bender, T. Conard, C. Zhao, A. Delabie, M. Caymax, S. De Gendt, M. Heyns, M. M. Viitanen, M. de Ridder, H. H. Brongersma, Y. Tamminga, T. Dao, T. de Win, M. Verheijen, M. Kaiser, and M. Tuominen, Journal of Applied Physics 96 (9), 4878 (2004).

${ }^{187}$ M. Brunet, H. Mafhoz Kotb, L. Bouscayrol, E. Scheid, M. Andrieux, C. Legros, and S. Schamm-Chardon, Thin Solid Films 519 (16), 5638 (2011).

${ }^{188}$ M. Filipescu, N. Scarisoreanu, V. Craciun, B. Mitu, A. Purice, A. Moldovan, V. Ion, O. Toma, and M. Dinescu, Applied Surface Science 253 (19), 8184 (2007).

${ }^{189}$ L. P. Borilo and L. N. Spivakova, American Journal of Materials Science 2 (4), 119 (2012).

${ }^{190}$ M. T. Soo, N. Prastomo, A. Matsuda, G. Kawamura, H. Muto, A. F. M. Noor, Z. Lockman, and K. Y. Cheong, Applied Surface Science 258 (13), 5250 (2012).

${ }^{191}$ Z. Ning-Lin, W. Qing, S. Zhi-Tang, S. Qin-Wo, Z. Xiang-Rong, and L. Cheng-Lu, Chinese physics letters 19 (3), 395 (2002).

${ }^{192}$ P. Gao, L. J. Meng, M. P. dos Santos, V. Teixeira, and M. Andritschky, Vacuum 56 (2), 143 (2000).

${ }^{193}$ G. Sethi, P. Sunal, M.W. Horn, and M. T. Lanagan, Journal of Vacuum Science \& Technology A 27 (3), 577 (2009).

${ }^{194}$ J. Y. Park, J. Y. Heo, and Y. C. Kang, Bull Korean Chem Soc 31, 397 (2010).

${ }^{195}$ X. Zhao, J. Jin, J. C. Cheng, J. W. Lee, K. H. Wu, K. C. Lin, J. R. Tsai, and K. C. Liu, Thin Solid Films 570, Part B, 404 (2014).

${ }^{196}$ J. Kleiman, Z. Iskanderova, Y. Gudimenko, and S. Horodetsky, in Materials in a Space Environment (2003), Vol. 540, pp. 313. 
${ }^{197}$ J. R. McNeil, J. J. McNally, and P. D. Reader, in Handbook of Thin-Film Deposition Processes and Techniques (2002), p. 463.

${ }^{198}$ M. S. Kwon and J. Y. Lee, Applied Surface Science 135 (1-4), 101 (1998).

${ }^{199}$ K. Reinhardt and W. Kern, Handbook of Silicon Wafer Cleaning Technology. (Elsevier Science, 2008).

${ }^{200}$ R. Garg, Ph.D. thesis, University at Albany, State University of New York, Albany, 2008.

${ }^{201}$ S. Bajt, H. N. Chapman, N. Nguyen, J. B. Alameda, J. C. Robinson, M. E. Malinowski, E. Gullikson, A. Aquila, C. Tarrio, and S. Grantham, in Emerging Lithographic Technologies VII (Proc. SPIE, Santa Clara, California, United States, 2003), Vol. 5037, pp. 236.

${ }^{202}$ A. Gao, P. J. Rizo, E. Zoethout, L. Scaccabarozzi, C. J. Lee, V. Banine, and F. Bijkerk, Journal of applied physics 114 (4), 044313 (2013).

${ }^{203}$ N. Koster, B. Mertens, R. Jansen, A. van de Runstraat, F. Stietz, Ma. Wedowski, H. Meiling, R. Klein, A. Gottwald, and F. Scholze, Microelectronic engineering 61, 65 (2002).

${ }^{204}$ M. E. Malinowski, C. A. Steinhaus, D. E. Meeker, W. M. Clift, L. E Klebanoff, and S. Bajt, in Microlithography 2003 (International Society for Optics and Photonics, 2003), pp. 429.

${ }^{205}$ R. Blume, H. Niehus, H. Conrad, A. Böttcher, L. Aballe, L. Gregoratti, A. Barinov, and M. Kiskinova, The Journal of Physical Chemistry B 109 (29), 14052 (2005).

${ }^{206}$ L. Belau, J. Y. Park, T. Liang, and G. A. Somorjai, Journal of Vacuum Science \& Technology B 26 (6), 2225 (2008).

${ }^{207}$ M. Nakahara, S. Tsunekawa, K. Watanabe, T. Arai, T. Yunogami, and K. Kuroki, Journal of Vacuum Science \& Technology B 19 (6), 2133 (2001).

${ }^{208}$ A. Chambers, Modern Vacuum Physics. (CRC Press, 2004).

${ }^{209}$ A. Bottcher and H. Niehus, The Journal of Chemical Physics 110 (6), 3186 (1999).

${ }^{210}$ K. Oura, V.G. Lifshits, A. Saranin, A.V. Zotov, and M. Katayama, Surface Science: An Introduction. (Springer Berlin Heidelberg, 2013).

${ }^{211}$ One monolayer (1 ML) corresponds to a coverage of as many absorbates on the surface as metal atoms in the topmost layer.

${ }^{212}$ I. Chorkendorff and J. W. Niemantsverdriet, in Concepts of Modern Catalysis and Kinetics (Wiley-VCH Verlag GmbH \& Co. KGaA, 2005), pp. 23.

${ }^{213}$ M. Tatarkhanov, F. Rose, E. Fomin, D. F. Ogletree, and M. Salmeron, Surface Science 602 (2), 487 (2008).

${ }^{214}$ R. van Harrevelt, K. Honkala, J. K Nørskov, and U. Manthe, The Journal of chemical physics 122 (23), 234702 (2005).

${ }^{215}$ W. J. Mitchell, J. Xie, K. J. Lyons, and W. H. Weinberg, Journal of Vacuum Science \& Technology A 12 (4), 2250 (1994).

${ }^{216}$ L. Surnev, G. Rangelov, and G. Bliznakov, Surface Science 159 (2), 299 (1985).

${ }^{217}$ K.W. Kolasinski, Surface Science: Foundations of Catalysis and Nanoscience. (Wiley, 2008).

${ }^{218}$ R. G. Compton, C. H. Bamford, and C. F. H. Tipper†, Simple Processes at the GasSolid Interface. (Elsevier Science, 1984).

${ }^{219}$ T. E. Madey, H. A. Engelhardt, and D. Menzel, Surface Science 48 (2), 304 (1975).

${ }^{220}$ S. L. Parrott, G. Praline, B. E. Koel, J. M. White, and T. N. Taylor, The Journal of Chemical Physics 71 (8), 3352 (1979).

${ }^{221}$ M. C. Wheeler, D. C. Seets, and C. B. Mullins, The Journal of Chemical Physics 105 (4), 1572 (1996). 
${ }^{222}$ R. Coloma Ribera, R.W.E van de Kruijs, J. M. Sturm, A. E. Yakshin, and F. Bijkerk, (to be published).

${ }^{223}$ B. Herd, M. Knapp, and H. Over, The Journal of Physical Chemistry C 116 (46), 24649 (2012).

${ }^{224}$ B. Herd and H. Over, Surface Science $622(0), 24$ (2014).

${ }^{225}$ K. Reuter, C. Stampfl, Verónica G. P. M., and M. Scheffler, Chemical Physics Letters 352 (5-6), 311 (2002).

${ }^{226}$ J. I. Flege, J. Lachnitt, D. Mazur, P. Sutter, and J. Falta, Physical Chemistry Chemical Physics 18 (1), 213 (2016).

${ }^{227}$ N. Cabrera and N. F. Mott, Reports on progress in physics 12 (1), 163 (1949).

${ }^{228}$ A. Atkinson, Reviews of Modern Physics 57 (2), 437 (1985).

${ }^{229}$ R. Blume, M. Hävecker, S. Zafeiratos, D. Teschner, E. Kleimenov, A. Knop-Gericke, R. Schlögl, A. Barinov, P. Dudin, and M. Kiskinova, Journal of Catalysis 239 (2), 354 (2006).

${ }^{230}$ Y. B. He, M. Knapp, E. Lundgren, and H. Over, The journal of physical chemistry. B 109 (46), 21825 (2005).

${ }^{231}$ R. Blume, M. Havecker, S. Zafeiratos, D. Teschner, E. Vass, P. Schnorch, A. KnopGericke, R. Schlogl, S. Lizzit, P. Dudin, A. Barinov, and M. Kiskinova, Physical chemistry chemical physics : PCCP 9 (27), 3648 (2007).

${ }^{232}$ H. Over and A. P. Seitsonen, Science 297 (5589), 2003 (2002).

${ }^{233}$ A. Böttcher and H. Niehus, Physical Review B 60 (20), 14396 (1999).

${ }^{234}$ H. Over, Y. B. He, A. Farkas, G. Mellau, C. Korte, M. Knapp, M. Chandhok, and M. Fang, Journal of Vacuum Science \& Technology B: Microelectronics and Nanometer Structures 25 (4), 1123 (2007).

${ }^{235}$ K. Thürmer, E. Williams, and J. Reutt-Robey, Science 297 (5589), 2033 (2002).

${ }^{236}$ L. P. H. Jeurgens, W. G. Sloof, F. D. Tichelaar, and E. J. Mittemeijer, Physical Review B 62 (7), 4707 (2000).

${ }^{237}$ H. Over, M. Knapp, Edvin. Lundgren, A. P. Seitsonen, M. Schmid, and P. Varga, ChemPhysChem 5 (2), 167 (2004).

${ }^{238} \mathrm{M}$. Rössler, S. Günther, and J. Wintterlin, The Journal of Physical Chemistry C 111 (5), 2242 (2007).

${ }^{239}$ J. D. Osorio, J. Giraldo, J. C. Hernández, A. Toro, and J. P. Hernández-Ortiz, Heat and Mass Transfer 50 (4), 483 (2014).

${ }^{240}$ A.T. Fromhold, Theory of Metal Oxidation. (North-Holland, 1976).

${ }^{241}$ N. B. Pilling and R. E. Bedworth, J. Inst. Met. 29, 529 (1923).

${ }^{242}$ A. T. Fromhold and E. L. Cook, Physical Review 175 (3), 877 (1968).

${ }^{243}$ V. B. Vykhodets, T. E. Kurennykh, A. G. Kesarev, M. V. Kuznetsov, V. V. Kondrat'ev, C. Hülsen, and U. Koester, JETP letters 93 (1), 5 (2011).

${ }^{244}$ F. J. Keneshea and D. L. Douglass, Oxidation of metals 3 (1), 1 (1971).

${ }^{245}$ R. T. Shannon, Acta Crystallographica Section A: Crystal Physics, Diffraction, Theoretical and General Crystallography 32 (5), 751 (1976).

${ }^{246}$ M. Martin, in Diffusion in Condensed Matter (Springer, 2005), pp. 209.

${ }^{247}$ A. V. Roshchin and V. E. Roshchin, Russian Metallurgy Metally c/c of IzvestiiaAkademiia Nauk SSSR Metally (1), 1 (2003).

${ }^{248}$ R. Stevens, An Introduction to Zirconia. (Magnesium Elektron Limited, 1983).

${ }^{249}$ G. Jomard, T. Petit, A. Pasturel, L. Magaud, G. Kresse, and J. Hafner, Physical Review B 59 (6), 4044 (1999).

${ }^{250}$ Z. Bastl, J. Židů, and K. Roháček, Thin Solid Films 213 (1), 103 (1992). 
${ }^{251}$ R. E. Smallman and A. H. W. Ngan, Modern Physical Metallurgy. (Elsevier Science, 2013).

${ }^{252}$ D. Ceresoli and D. Vanderbilt, Physical Review B 74 (12), 125108 (2006).

${ }^{253}$ H. Mehrer, Diffusion in Solids: Fundamentals, Methods, Materials, DiffusionControlled Processes. (Springer, 2007).

${ }^{254}$ K. Shimakawa and M. Aniya, Monatsh Chem 144 (1), 67 (2013).

${ }^{255}$ W. Meyer and H. Neldel, Zeitschrift für Technische Physik (Leipzig) 12, 588 (1937).

${ }^{256}$ A. Yelon, B. Movaghar, and R. S. Crandall, Reports on Progress in Physics 69 (4), 1145 (2006).

${ }^{257}$ R. Widenhorn, A. Rest, and E. Bodegom, Journal of Applied Physics 91 (10), 6524 (2002).

${ }^{258}$ A. W. Lawson, The Journal of Chemical Physics 32 (1), 131 (1960).

${ }^{259}$ A. Juma, H. Wafula, E. Wendler, and T. Dittrich, Journal of Applied Physics 115 (5), 053703 (2014).

${ }^{260}$ G. Boisvert, L. J. Lewis, and A. Yelon, Physical Review Letters 75 (3), 469 (1995).

${ }^{261}$ A. Yelon and B. Movaghar, Physical Review B 65 (7), 077202 (2002).

${ }^{262}$ C. Stampfl, S. Schwegmann, H. Over, M. Scheffler, and G. Ertl, Physical Review Letters 77 (16), 3371 (1996).

${ }^{263}$ Y. D. Kim, S. Wendt, S. Schwegmann, H. Over, and G. Ertl, Surface Science 418 (1), 267 (1998).

${ }^{264}$ H. Over, Progress in Surface Science 58 (4), 249 (1998).

${ }^{265}$ Y. B. He, A. Goriachko, C. Korte, A. Farkas, G. Mellau, P. Dudin, L. Gregoratti, A. Barinov, M. Kiskinova, A. Stierle, N. Kasper, S. Bajt, and H. Over, The Journal of Physical Chemistry C 111 (29), 10988 (2007).

${ }^{266}$ Z. Li, T. Schram, L. Pantisano, T. Conard, S. Van Elshocht, W. Deweerd, S. De Gendt, K. De Meyer, A. Stesmans, S. Shamuilia, V. V. Afanas'ev, A. Akheyar, D. P. Brunco, N. Yamada, and P. Lehnen, Journal of Applied Physics 101 (3) (2007).

${ }^{267}$ E. V. Jelenkovic and K. Y. Tong, Journal of Vacuum Science \& Technology B: Microelectronics and Nanometer Structures 22 (5), 2319 (2004).

${ }^{268}$ C. V. Ramana, S. Utsunomiya, R. C. Ewing, U. Becker, V. V. Atuchin, V. Sh. Aliev, and V. N. Kruchinin, Applied Physics Letters 92 (1), 011917 (2008).

${ }^{269}$ H. I. Ji, J. Hwang, K. J. Yoon, J. W. Son, B. K. Kim, H. W. Lee, and J. H. Lee, Energy \& Environmental Science 6 (1), 116 (2013).

${ }^{270}$ H. H. Brongersma, M. Draxler, M. de Ridder, and P. Bauer, Surface Science Reports 62 (3), 63 (2007).

${ }^{271}$ J. Geiser and S. Blankenburg, Communications in Computational Physics 11 (5), 1618 (2012).

${ }^{272}$ P. Kotnik, P. Hofbauer, R. Resel, M. Koini, T. Haber, and J. Keckes, Acta Crystallographica Section A 62 (a1), s158 (2006).

${ }^{273}$ R. Resel, E. Tamas, B. Sonderegger, P. Hofbauer, and J. Keckes, Journal of Applied Crystallography 36 (1), 80 (2003).

${ }^{274}$ G. Koster and G. Rijnders, In Situ Characterization of Thin Film Growth. (Elsevier Science, 2011).

${ }^{275}$ G. Bracco and B. Holst, Surface Science Techniques. (Springer Berlin Heidelberg, 2013).

${ }^{276}$ H. H. Brongersma, in Characterization of Materials (John Wiley \& Sons, Inc., 2012).

${ }^{277}$ H. H. Brongersma, A. Gildenpfennig, A. W. Denier van der Gon, R. D. van de Grampel, W. P. A. Jansen, A. Knoester, J. Laven, and M. M. Viitanen, Nuclear 
Instruments and Methods in Physics Research Section B: Beam Interactions with Materials and Atoms 190 (1-4), 11 (2002).

${ }^{278}$ C. V. Cushman, P. Bruner, J. Zakel, G. H. Major, B. M. Lunt, N. J. Smith, T. Grehl, and M. R. Linford, Analytical Methods 8 (17), 3419 (2016).

${ }^{279}$ H. H. Brongersma, T. Grehl, P. A. van Hal, N. C. W. Kuijpers, S. G. J. Mathijssen, E. R. Schofield, R. A. P. Smith, and H. R. J. ter Veen, Vacuum 84 (8), 1005 (2010).

${ }^{280}$ J.F. Watts and J. Wolstenholme, An Introduction to Surface Analysis by XPS and AES. (Wiley, 2003).

${ }^{281}$ Peter J Cumpson, Journal of electron spectroscopy and related phenomena 73 (1), 25 (1995).

${ }^{282}$ P. J. Cumpson and M. P. Seah, Surface and Interface Analysis 25 (6), 430 (1997).

${ }^{283}$ P. Hones, T. Gerfin, and M. Gratzel, Applied Physics Letters 67 (21), 3078 (1995).

${ }^{284}$ P. Hones, F. Lévy, T. Gerfin, and M. Grätzel, Chemical Vapor Deposition 6 (4), 193 (2000).

${ }^{285}$ D. Dale, Y. Suzuki, and J. D. Brock, Journal of Physics: Condensed Matter 20 (26), 264008 (2008).

${ }^{286}$ R. Pascu and M. Dinescu, Romanian Reports in Physics 64 (1), 135 (2012).

${ }^{287}$ B. L. Henke, E. M. Gullikson, and J. C. Davis, Atomic Data and Nuclear Data Tables 54 (2), 181 (1993).

${ }^{288}$ K. N. Stoev and K. Sakurai, Spectrochimica Acta Part B: Atomic Spectroscopy 54 (1), 41 (1999).

${ }^{289}$ W. R. Leo, Techniques for Nuclear and Particle Physics Experiments: A How-to Approach. (Springer Berlin Heidelberg, 2012).

${ }^{290}$ E. Hecht, Optics 2nd edition ed. (Addison-Wesley Publishing Company, 1987).

${ }^{291}$ M. Yasaka, The Rigaku Journal 26 (2) (2010).

${ }^{292}$ W. H. Bragg and W. L. Bragg, Proceedings of the Royal Society of London. Series A 88 (605), 428 (1913).

${ }^{293}$ H. Kiessig, Annals of Physics 10, 715 (1931).

${ }^{294}$ M. Bjorck and G. Andersson, Journal of Applied Crystallography 40 (6), 1174 (2007).

${ }^{295}$ L. G. Parratt, Physical Review 95 (2), 359 (1954).

${ }^{296}$ L. Névot and P. Croce, Rev. Phys. Appl. (Paris) 15 (3), 761 (1980).

${ }^{297} \mathrm{G}$. Cappuccio and Associazione Italiana di Cristallografia, Thin film characterisation by advanced X-ray diffraction techniques : Frascati, October 2-5, 1996 : V School on $X$-ray Diffraction from Polycrystalline Materials. (Laboratori Nazionali di Frascati, Frascati (Italy), 1996).

${ }^{298}$ Y. Xu, M. Yamazaki, and P. Villars, Japanese Journal of Applied Physics 50 (11S), 11RH02 (2011).

${ }^{299}$ J. O. A. Paschoal, H. Kleykamp, and F. Thümmler, Journal of the Less Common Metals 98 (2), 279 (1984).

${ }^{300}$ P. Scherrer, in Kolloidchemie Ein Lehrbuch (Springer, 1912), pp. 387.

${ }^{301}$ A. L. Patterson, Physical Review 56 (10), 978 (1939).

${ }^{302}$ J. I. Langford and A. J. C. Wilson, Journal of Applied Crystallography 11 (2), 102 (1978).

${ }^{303} \mathrm{~T}$. Roisnel and J. Rodríquez-Carvajal, in Materials Science Forum (Transtec Publications; 1999, 2001), Vol. 378, pp. 118.

${ }^{304} \mathrm{G}$. Haugstad, Atomic force microscopy: understanding basic modes and advanced applications. (John Wiley \& Sons, 2012).

${ }^{305}$ B. Cappella and G. Dietler, Surface Science Reports 34 (1), 1 (1999). 
${ }^{306}$ I. Horcas, R. Fernández, J. M. Gomez-Rodriguez, J. Colchero, J. W. S. X. M. GómezHerrero, and A. M. Baro, Review of Scientific Instruments 78 (1), 013705 (2007).

${ }^{307}$ R. Erni, M. D. Rossell, C. Kisielowski, and U. Dahmen, Physical Review Letters 102 (9), 096101 (2009).

${ }^{308}$ D.B. Williams and C.B. Carter, Transmission Electron Microscopy: A Textbook for Materials Science. (Springer US, 2013).

${ }^{309}$ W. L. Sarney, Sample Preparation Procedure for TEM Imaging of Semiconductor Materials. (Army Research Laboratory, 2004).

${ }^{310}$ Gatan et. al., Inc., DigitalMicrograph, version 2.3, 2013, see http://www.gatan.com.

${ }^{311}$ H. Over, Y. D. Kim, A. P. Seitsonen, S. Wendt, E. Lundgren, M. Schmid, P. Varga, A. Morgante, and G. Ertl, Science 287 (5457), 1474 (2000).

${ }^{312}$ S. Trasatti, Electrochimica Acta 45 (15-16), 2377 (2000).

${ }^{313}$ T. E. Madey, H. Albert Engelhardt, and D. Menzel, Surface Science 48 (2), 304 (1975).

${ }^{314}$ C. Corriol, F. Calleja, A. Arnau, J. J. Hinarejos, A. L. Vázquez de Parga, W. A. Hofer, and R. Miranda, Chemical Physics Letters 405 (1-3), 131 (2005).

${ }^{315}$ S. Poulston, M. Tikhov, and R. M. Lambert, Langmuir 13 (20), 5356 (1997).

${ }^{316}$ M. Knapp, A. P. Seitsonen, Y. D. Kim, and H. Over, The Journal of Physical Chemistry B 108 (38), 14392 (2004).

${ }^{317}$ L. Perring, F. Bussy, J. C. Gachon, and P. Feschotte, Journal of Alloys and Compounds 284 (1-2), 198 (1999).

${ }^{318}$ G. Borzone, R. Raggio, and R. Ferro, J Min Met 38, 249 (2002).

${ }^{319}$ Y. S. Huang, H. L. Park, and F. H. Pollak, Materials Research Bulletin 17 (10), 1305 (1982).

${ }^{320}$ H. Over, A. P. Seitsonen, E. Lundgren, M. Smedh, and J. N. Andersen, Surface Science 504 (0), L196 (2002).

${ }^{321}$ K. Reuter and M. Scheffler, Physical Review B 65 (3), 035406 (2001).

${ }^{322} \mathrm{H}$. Zhong and North Carolina State University, Ruthenium-based Gate Electrodes for Advanced Dual-metal Gate CMOS Devices. (North Carolina State University, 2002).

${ }^{323}$ T. Aoyama, S. Yamazaki, and K. Imai, Japanese Journal of Applied Physics 39 (Part 1, No. 11), 6348 (2000).

${ }^{324}$ J. Assmann, V. Narkhede, N. A. Breuer, M. Muhler, M. Knapp, D. Crihan, A. Farkas, G. Mellau, and H. Over, Journal of Physics: Condensed Matter 20 (18), 184017 (2008).

${ }^{325}$ R. Coloma Ribera, R. W. E. van de Kruijs, S. Kokke, E. Zoethout, A. E. Yakshin, and F. Bijkerk, Applied Physics Letters 105 (13), 131601 (2014).

${ }^{326}$ A. Kumar and A. Kumar, Bull Mater Sci 38 (1), 41 (2015).

${ }^{327}$ J. C. Palacios, M. G. Olayo, G. J. Cruz, and J. A. Chávez-Carvayar, Open Journal of Polymer Chemistry 4, 31 (2014).

${ }^{328}$ S. Arlinghaus, Practical Handbook of Curve Fitting. (Taylor \& Francis, 1994).

${ }^{329} \mathrm{C}$. Walck, Handbook on statistical distributions for experimentalists. (2007).

${ }^{330}$ N. Saadatjou, A. Jafari, and S. Sahebdelfar, Chemical Engineering Communications 202 (4), 420 (2015).

${ }^{331}$ H. Kim, T. Koseki, T. Ohba, T. Ohta, Y. Kojima, H. Sato, and Y. Shimogaki, Journal of The Electrochemical Society 152 (8), G594 (2005).

${ }^{332}$ G. A. Adegboyega and A. Poggi, physica status solidi (a) 143 (2), 373 (1994).

${ }^{333}$ R. Chan, T. N. Arunagiri, Y. Zhang, O. Chyan, R. M. Wallace, M. J. Kim, and T. Q. Hurd, Electrochemical and Solid-State Letters 7 (8), G154 (2004).

${ }^{334}$ R. Coloma Ribera, R. W. E. van de Kruijs, A. E. Yakshin, and F. Bijkerk, Journal of Applied Physics 118 (5), 055303 (2015). 
${ }^{335}$ P.Y. Yan, E. Spiller, and P. Mirkarimi, Journal of Vacuum Science \& Technology B 25 (6), 1859 (2007).

${ }^{336}$ L. S. Zambom, R. D. Mansano, and A. P. Mousinho, Microelectronics Journal 40 (1), 66 (2009).

${ }^{337}$ A. P. Mousinho, R. D. Mansano, L. S. Zambom, and A. Passaro, Journal of Physics: Conference Series 370 (1), 012015 (2012).

${ }^{338}$ A. Rafati, R. ter Veen, and D. G. Castner, Surface and Interface Analysis 45 (11-12), 1737 (2013).

${ }^{339}$ H. Tellez, A. Aguadero, J. Druce, M. Burriel, S. Fearn, T. Ishihara, D. S. McPhail, and J. A. Kilner, Journal of Analytical Atomic Spectrometry 29 (8), 1361 (2014).

${ }^{340}$ L. Vegard, Zeitschrift für Physik 5 (1), 17 (1921).

${ }^{341}$ L. C. A. van den Oetelaar, H. E. van Benthem, J. H. J. M. Helwegen, P. J. A. Stapel, and H. H. Brongersma, Surface and Interface Analysis 26 (8), 537 (1998).

${ }^{342}$ W.H. Greene, Econometric Analysis. (Pearson/Prentice Hall, 2008).

${ }^{343}$ B. Vidal and P. Vincent, Applied Optics 23 (11), 1794 (1984).

${ }^{344}$ V. I. T. A. de Rooij-Lohmann, A. W. Kleyn, F. Bijkerk, H. H. Brongersma, and A. E. Yakshin, Applied Physics Letters 94 (6), 063107 (2009).

${ }^{345}$ M. de Ridder and H. H. Brongersma, Private communication. (2006).

${ }^{346}$ H. Maury, J. M. André, K. Le Guen, N. Mahne, A. Giglia, S. Nannarone, F. Bridou, F. Delmotte, and P. Jonnard, Surface Science 603 (2), 407 (2009).

${ }^{347}$ W. H. Wang and W. K. Wang, Journal of Materials Research 9 (02), 401 (1994).

${ }^{348}$ P. K. Hucknall, C. G. H. Walker, D. Greig, J. A. D. Matthew, D. Norman, and J. Turton, Surface and Interface Analysis 19 (1-12), 23 (1992).

${ }^{349}$ V. I. T. A. de Rooij-Lohmann, A. E. Yakshin, E. Zoethout, J. Verhoeven, and F. Bijkerk, Applied Surface Science 257 (14), 6251 (2011).

${ }^{350}$ L. Z. Mezey and J. Giber, Japanese Journal of Applied Physics 21 (11R), 1569 (1982).

${ }^{351}$ R. W. E. van de Kruijs, E. Zoethout, A. E. Yakshin, I. Nedelcu, E. Louis, H. Enkisch,

G. Sipos, S. Müllender, and F. Bijkerk, Thin Solid Films 515 (2), 430 (2006).

${ }^{352}$ F. Liu, J. M. Sturm, C. J. Lee, and F. Bijkerk, Surface Science 646, 101 (2016).

${ }^{353}$ R. Coloma Ribera, R. W. E. van de Kruijs, J. M. Sturm, A. E. Yakshin, and F. Bijkerk, Journal of Applied Physics 120 (6), 065303 (2016).

${ }^{354}$ G. Balakrishnan, P. Kuppusami, D. Sastikumar, and J I. Song, Nanoscale Research Letters 8 (1), 82 (2013).

${ }^{355}$ H. Döscher, G. Lilienkamp, P. Iskra, W. Daum, G. Helsch, S. Becker, R. J. Wrobel, H. Weiss, and Y. Suchorski, Journal of Applied Physics 107 (9), 094103 (2010).

${ }^{356}$ A. Kumar, S. Mondal, and K. S. R. K. Rao, Applied Surface Science 370, 373 (2016).

${ }^{357}$ S. Jena, R. B. Tokas, S. Thakur, and N. K. Sahoo, Indian Journal of Physics 90 (8), 951 (2016).

${ }^{358}$ Thermo Fisher Scientific et. al., Inc., Thermo Avantage, version 5.938, a surface analysis software, 2014, see http://www.thermoscientific.com.

${ }^{359}$ J. Végh, Journal of Electron Spectroscopy and Related Phenomena 46 (2), 411 (1988).

${ }^{360}$ J. H. Scofield, Journal of Electron Spectroscopy and Related Phenomena 8 (2), 129 (1976).

${ }^{361}$ O.T. Soerensen, Nonstoichiometric Oxides. (Elsevier Science, 2012).

${ }^{362}$ M. Gutowski, J. E. Jaffe, C. L. Liu, M. Stoker, R. I. Hegde, R. S. Rai, and P. J. Tobin, Applied Physics Letters 80 (11), 1897 (2002).

${ }^{363}$ C. C. Fulton, T. E. Cook, G. Lucovsky, and R. J. Nemanich, Journal of Applied Physics 96 (5), 2665 (2004). 
${ }^{364}$ G. B. Rayner, D. Kang, Y. Zhang, and G. Lucovsky, Journal of Vacuum Science \& Technology B 20 (4), 1748 (2002).

${ }^{365}$ J. J. Zuckerman and A. P. Hagen, Inorganic Reactions and Methods, The Formation of Bonds to Elements of Group IVB (C, Si, Ge, Sn, Pb). (Wiley, 2009).

${ }^{366}$ J. A Van Orman and K. L Crispin, Reviews in Mineralogy and Geochemistry 72 (1), 757 (2010).

${ }^{367}$ R. L. Opila, G. D. Wilk, M. A. Alam, R. B. van Dover, and B. W. Busch, Applied Physics Letters 81 (10), 1788 (2002).

${ }^{368}$ J. D. Ingle and S. R. Crouch, Spectrochemical Analysis. (Prentice Hall, 1988).

${ }^{369}$ J. F. Watts and J. Wolstenholme, in An Introduction to Surface Analysis by XPS and AES (John Wiley \& Sons, Ltd, 2005), pp. 79.

${ }^{370}$ A. Hardy, S. Van Elshocht, C. Adelmann, T. Conard, A. Franquet, O. Douhéret, I. Haeldermans, J. D'Haen, S. De Gendt, M. Caymax, M. Heyns, M. D'Olieslaeger, M.K. Van Bael, and J. Mullens, Thin Solid Films 516 (23), 8343 (2008).

${ }^{371}$ P. J. van Zwol, D. F. Vles, W. P. Voorthuijzen, M. Péter, H. Vermeulen, W. J. van der Zande, J. M. Sturm, R. W. E. van de Kruijs, and F. Bijkerk, Journal of Applied Physics 118 (21), 213107 (2015). 




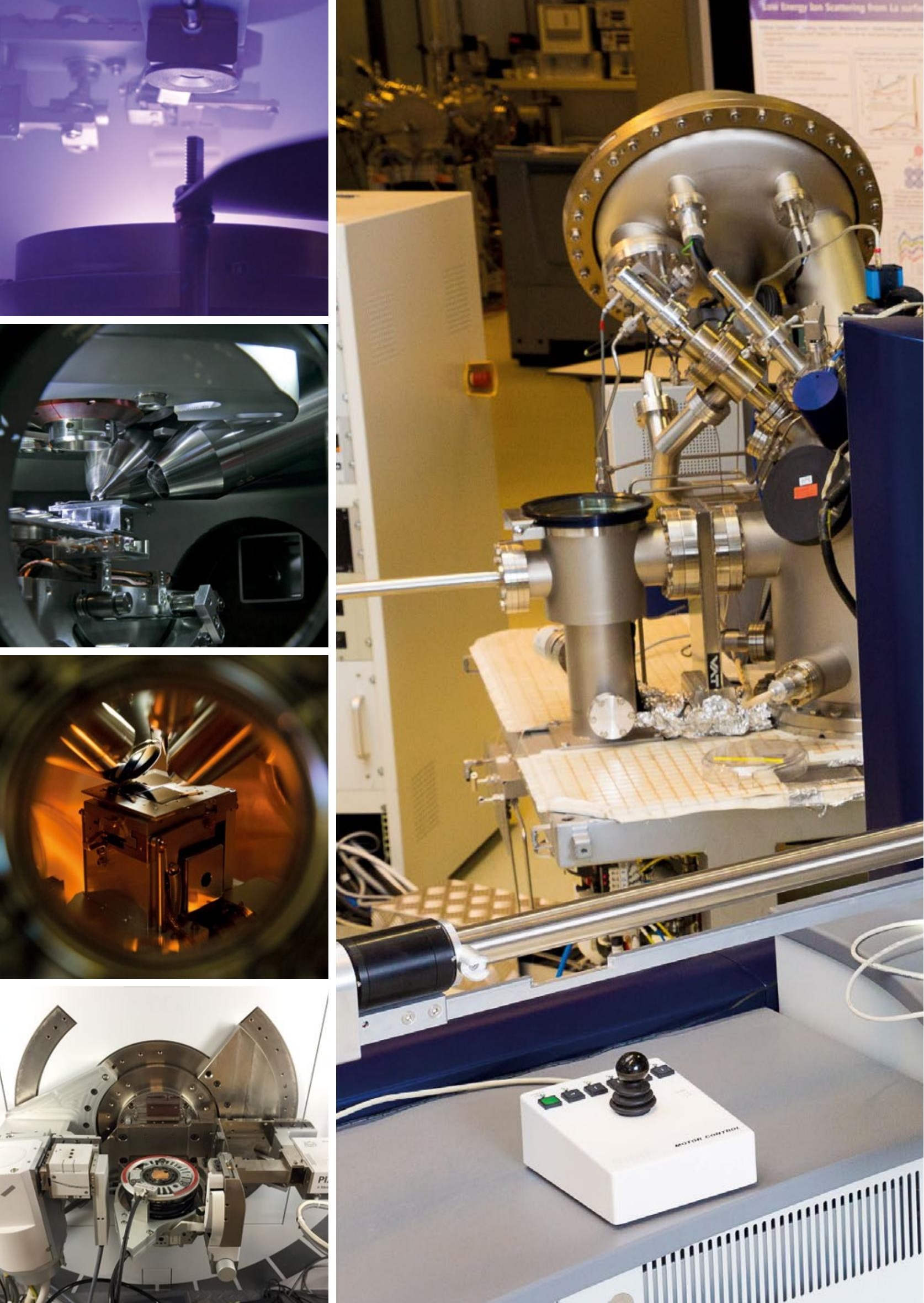

